\title{
Das elektrochemische Potential auf der atomaren Skala: \\ Untersuchung des Ladungstransports eines stromtragenden zweidimensionalen Elektronengases mit Hilfe der Raster-Tunnel-Potentiometrie
}

\author{
Dissertation \\ zur Erlangung des Doktorgrades \\ der Mathematisch-Naturwissenschaftlichen Fakultäten \\ der Georg-August-Universität zu Göttingen
}

vorgelegt von

Jan Homoth, geb. Müller

aus Göttingen

Göttingen 2008 
D 7

Referent:

Prof. Dr. R. G. Ulbrich

Korreferent:

Prof. Dr. K. Samwer

Tag der mündlichen Prüfung: $\quad$ 3. Juli 2008 



\section{Einleitung}

2007 wurde erstmals ein funktionierendes Radio basierend auf einem einzigen Molekül (Kohlenstoff-Nanoröhre) mit 500 Nanometern Länge demonstriert [1-3] und die internationale „Roadmap for Semiconductors“ sagt voraus, dass Transistoren als Grundbaustein eines jeden Computers in den nächsten Jahren Strukturgrößen bis hinunter zu 10 Nanometern erreichen werden [4]. Dies sind zwei von unzähligen Beispielen für die voranschreitende Miniaturisierung elektronischer Bauelemente. Bereits jetzt ist ein Miniaturisierungsgrad erreicht, bei dem die diskrete atomare Struktur der stromtragenden Materie über die Funktionalität eines Bauelements entscheidet [5]. Der elektrische Stromfluss durch derart kleine Strukturen kann von makroskopischen Gesetzmäßigkeiten abweichen und zu überraschenden Ergebnissen führen. Zum Beispiel führt eine Halbierung eines makroskopischen Widerstands zu einer Halbierung der an den Widerstandsteilen abfallenden Spannung, was auf der mikroskopischen Skala nicht mehr gilt. ${ }^{l}$

Für makroskopische Festkörper wird der lineare Zusammenhang von Stromdichte $\vec{j}$ und elektrischem Feld $\vec{E}$ durch das Ohmsche Gesetz $\vec{j}=\hat{\sigma} \vec{E}$ beschrieben, wobei $\hat{\sigma}$ die elektrische Leitfähigkeit ist. Mikroskopisch betrachtet ist $\hat{\sigma}$ im Allgemeinen jedoch ein komplexes Funktional $\hat{\sigma}=\hat{\sigma}(\vec{r}, \vec{E}, \vec{j}, \ldots)$ und das Ohmsche Gesetz resultiert aus der räumlichen Mittelung über eine Vielzahl der zugrundeliegenden mikroskopischen Prozesse. Je nach betrachteter Längenskala wird der Ladungstransport in unterschiedlichen konzeptionellen Modellen diskutiert. Auf der mikroskopischen Skala dominieren quantenmechanische Effekte den Transport, wohingegen bei makroskopischen Festkörpern eine diffuse Modellierung im Rahmen des Drude-Sommerfeld-Modells geeignet sein kann.

1 Bei dieser Beschreibung wurde absichtlich von dem umgangssprachlich verwendeten Begriff der „elektrischen Spannung“ Gebrauch gemacht, um an das aus dem physikalischen Alltag bekannte Resultat einer variierenden elektrischen Spannung über einem Schleifwiderstand anzuknüpfen. Im Verlauf dieser Arbeit wird sich herausstellen, dass der elektrische Transport besser im Rahmen des elektrochemischen Potentials diskutiert werden sollte. 
Abbildung 1 zeigt als ein Ergebnis dieser Arbeit, die räumlich aufgelöste Spannungsverteilung innerhalb eines sehr dünnen, stromtragenden Films. Neben Bereichen, in denen die elektrische Spannung kontinuierlich variiert und somit im Sinne des Ohmschen Gesetzes skalierbar wäre, variiert die elektrische Spannung in anderen Bereichen auf kleinster Längenskala. Der makroskopisch beobachtbare Spannungsabfall resultiert aus der Summe dieser mikroskopischen Variationen. Für eine konzeptionelle Beschreibung des Ladungstransports von der atomaren bis hin zur makroskopischen Skala ist es daher notwendig, die zur Charakterisierung und zum Verständnis der Transporteigenschaften wichtigen physikalischen Größen auf diesen Längenbereichen zu analysieren. Man benötigt eine Sonde, die beim Ladungstransport das elektrostatische und elektrochemische Potential sowie die Besetzungsfunktionen zusammen mit den strukturellen Informationen mit hoher Ortsauflösung erfassen kann.
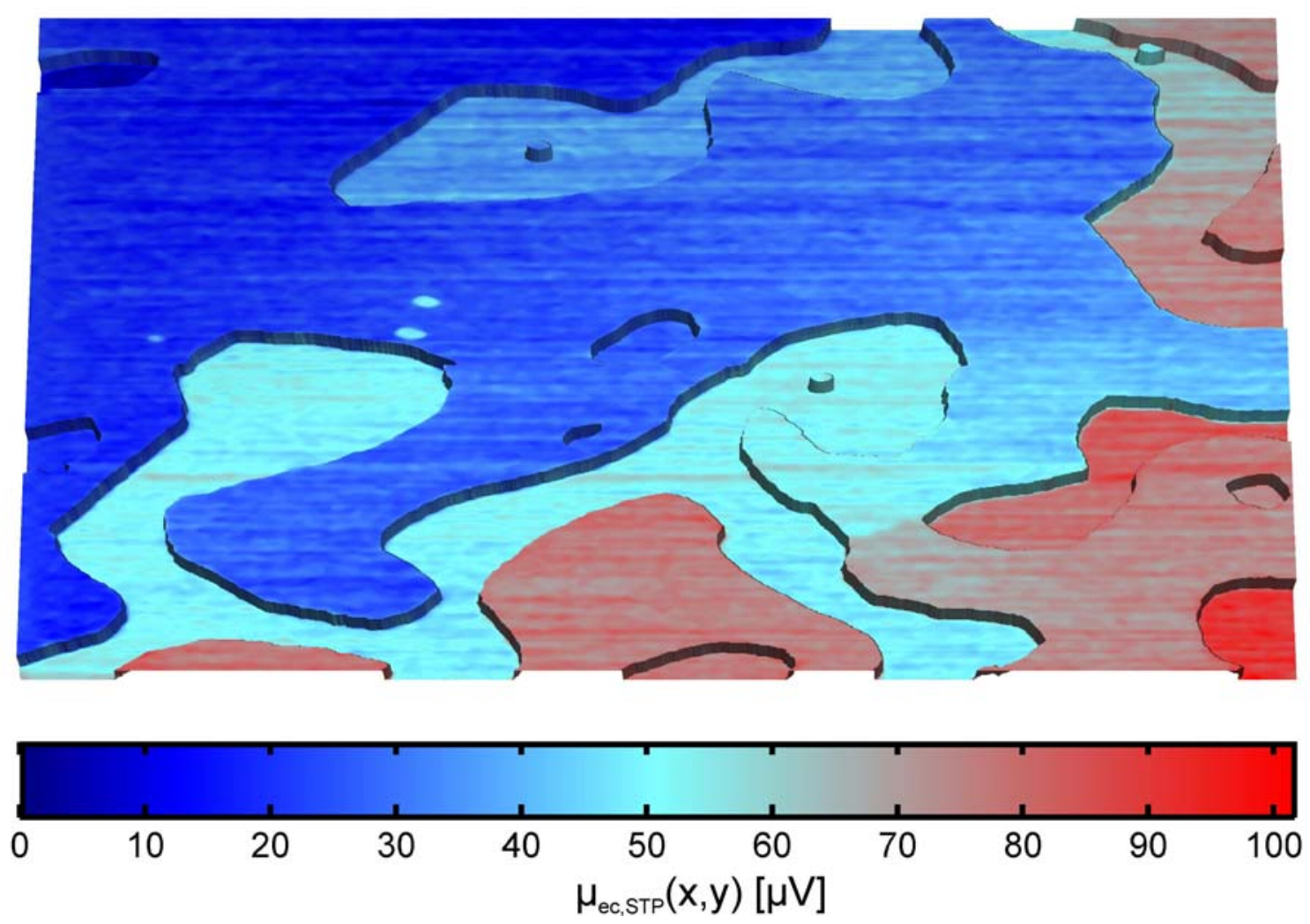

Abbildung 1: 3D-Darstellung (400 $\left.\times 400 \mathrm{~nm}^{2}\right)$ des elektrischen Spannungsverlaufs auf der Si(111)( $\sqrt{3} x \sqrt{ } 3)-A g$ Rekonstruktion. Die elektrische Spannung ist als Farbkodierung der Höheninformation überlagert. An monoatomaren Terrassenstufen variiert die elektrische Spannung auf kurzer Längenskala, während auf den freien Flächen homogene elektrische Felder vorherrschen. 
Neben der Elektronen-Holografie, die ausschließlich auf elektrostatische Felder sensitiv ist und noch auflösungsbedingte Schwächen besitzt, kommt diesem Anspruch nur die im weiteren Verlauf dieser Arbeit verwendete Raster-Tunnel-Potentiometrie (engl. Scanning Tunneling Potentiometry, STP) nah [6]. Sie wurde von Muralt und Pohl 1986 entwickelt, um das elektrische Potential in stromtragenden Strukturen mit hoher Ortsauflösung untersuchen zu können [7]. Hierzu wird der Probe ein elektrischer Strom aufgeprägt, der zu einer lateral variierenden elektrischen Spannung führen kann. Da das elektrische Potential der Tunnelspitze während des Abtastens der Probe stets bekannt ist, kann zusätzlich zu den strukturellen Eigenschaften die lokale Spannung der Probe am Ort der Tunnelspitze bestimmt werden.

Obwohl der 20 jährige Geburtstag der Raster-Tunnel-Potentiometrie bereits verstrichen ist, gibt es derzeit weltweit nur zwei Arbeitsgruppen, die STP mit atomarer Auflösung anwenden können. Die Anzahl sämtlicher Publikationen mit dieser Technik ist daher bislang sehr gering [7-22]. Woran liegt das? Der Widerstand des Tunnelkontakts ist typischerweise in der Größenordnung $10^{9} \Omega$. Legt man an die Enden einer Probe von $10 \mathrm{~mm}$ Länge ein Potentialunterschied von $10 \mathrm{~V}$, so ist das mittlere elektrische Feld innerhalb der Probe von der Größenordnung von $1 \mathrm{mV} / \mu \mathrm{m}$ oder $1 \mu \mathrm{V} / \mathrm{nm}$. Bei festem Tunnelwiderstand bedeutet dies eine laterale Variation des Tunnelstroms von nur $1 \mathrm{pA} / \mu \mathrm{m}$ oder aber $1 \mathrm{fA} / \mathrm{nm}$, was eine große Herausforderung an alle Komponenten des Messaufbaus (mechanisch und elektrisch) darstellt. Darüber hinaus liegt für gute Leiter, wie z. B. Kupfer, die mit diesem elektrischen Feld assoziierte Stromdichte weit über der Zerstörungsschwelle. So müsste für einen Spannungsgradienten von $1 \mu \mathrm{V} / \mathrm{nm}$ ein Draht von $1 \mathrm{~mm}$ Durchmesser einer Stromstärke von etwas über 1000 A widerstehen, so dass auch das Probensystem selbst eine große experimentelle Herausforderung darstellt. Das Zusammenspiel beider Aspekte ist wahrscheinlich der Grund für die geringe Anzahl an Raster-Tunnel-Potentiometrie Ergebnissen auf kleinster Längenskala. 
Konkret wird in dieser Arbeit der Ladungstransport durch das zweidimensionale Elektronengas der $\operatorname{Si}(111)(\sqrt{ } 3 x \sqrt{ } 3)$-Ag Rekonstruktion als prototypisches Modellsystem untersucht. Der Ladungstransport durch dieses System findet direkt an der Oberfläche eines Kristalls statt, so dass alle relevanten Parameter mit Hilfe der Raster-Tunnel-Potentiometrie analysiert werden können. Hierfür wird das elektrochemische Potential, als Kenngröße für den Ladungstransport, zusammen mit strukturellen Informationen vermessen. Die mikroskopischen Prozesse, welche sich im komplexen Zusammenspiel zum makroskopisch beobachtbaren Ohmschen Gesetzt aufsummieren, werden erstmalig mit höchster Ortsauflösung von bis zu $2 \cdot 10^{-10}$ Meter im Detail studiert. Beiträge von lokalisierter Streuung an Defekten und diffuser ElektronPhonon Streuung werden beobachtet. Die gemachten Experimente decken darüber hinaus den Übergang von der mikroskopischen zur makroskopischen Skala ab und zeigen, wie sich die elementaren Prozesse zum makroskopisch beobachteten Widerstand auf summieren.

Kapitel 1 führt in die theoretischen und experimentellen Grundlagen ein. Insbesondere wird hier der Messprozess der Raster-Tunnel-Potentiometrie vorgestellt.

Kapitel 2 beginnt mit einer Zusammenfassung der bisherigen Erkenntnisse über die $\operatorname{Si}(111)(\sqrt{3} x \sqrt{3})$-Ag Rekonstruktion. Die Präparation und Kontaktierung des zweidimensionalen Elektronengases der $\operatorname{Si}(111)(\sqrt{3} \times \sqrt{3})$-Ag Rekonstruktion wird in Abschnitt 2.2 vorgestellt. In Abschnitt 2.3 werden alle transportrelevanten Eigenschaften, der im weiteren Verlauf dieser Arbeit verwendeten Proben gebündelt dargestellt. Einige Ergebnisse weichen bereits von der gängigen Literaturmeinung zu Beginn dieser Arbeit ab und werden entsprechend diskutiert.

Experimentelle Ergebnisse der Raster-Tunnel-Potentiometrie Messungen stellt Kapitel 3 vor und dem Ladungstransport durch das natürliche Perkollationsnetzwerk widmet sich Abschnitt 3.2. Nach einer generellen Einführung in die räumliche Verteilung des elektrochemischen Potentials wird in Abschnitt 3.2.1 die elektrische Leitfähigkeit durch monoatomare Substratstufen direkt aus den STP-Daten quantifiziert. Diese spezifische Stufenleitfähigkeit dient als Grundlage, um in Abschnitt 3.2.2 eine Modellierung der Transporteigenschaften auf Basis der vermessenen Realraumstruktur der Probe anzufertigen. Mit Hilfe dieser Modellierung gelingt es, die mikroskopischen Prozesse $\mathrm{zu}$ separieren und ihnen spezifische Eigenschaften zuzuweisen. Nachdem die spezifischen, mikroskopischen Transporteigenschaften identifiziert sind, wird der Übergang von mikroskopischen Transporteigenschaften zur makroskopischen Leitfähigkeit der Probe vollzogen. 
In Abschnitt 3.3 werden Ergebnisse zum Transport durch eine vizinale Oberfläche vorgestellt, welche in Zusammenarbeit mit der Arbeitsgruppe von Prof. Möller an der Universität EssenDuisburg untersucht worden ist. Das freie Elektronengas der $\operatorname{Si}(111)(\sqrt{3} \times \sqrt{3})$-Ag Rekonstruktion wurde hier zu durch monoatomare Substratstufen einer vizinalen Si(111)-Oberfläche zu einer Art Kanalstruktur lateral eingeengt. Auf Basis dieser Daten werden mikroskopische Transporteigenschaften extrahiert, die über ein Modell mit spezifischen Transmissivitäten und Leitfähigkeiten hinausgehen.

In Kapitel 4 wird der mikroskopische, räumliche Verlauf in der Umgebung eines lokalisierten Streuzentrums auf kleinster Skala untersucht. Der gemessene Verlauf des elektrochemischen Potentials an einer monoatomaren Substratstufe wird im Zusammenspiel mit STP-Simulation als Eigenschaft des Ladungstransports identifiziert und von Abbildungsartefakten unterschieden. Hieraus ergibt sich ein Interpretationsansatz, der über die verfügbaren Modellierungen des Ladungstransports hinaus geht.

Eine abschließende Zusammenfassung dieser Arbeit wird in Kapitel 5 vorgestellt und Anhang A diskutiert Konsistenzuntersuchungen zur Transportmodellierung. 


\section{Inhaltsverzeichnis}

Einleitung II

Inhaltsverzeichnis 1

1 Theoretische und experimentelle Grundlagen ................................................ 3

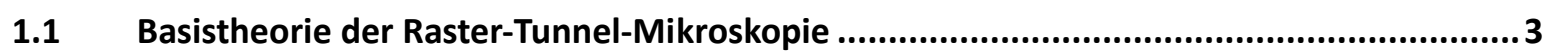

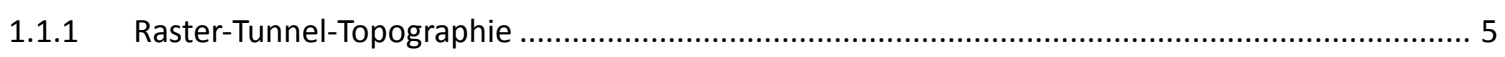

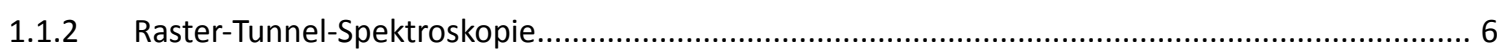

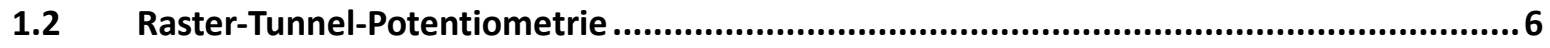

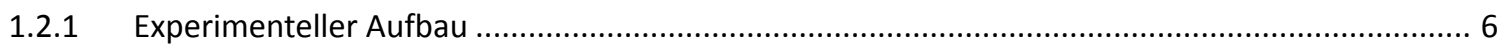

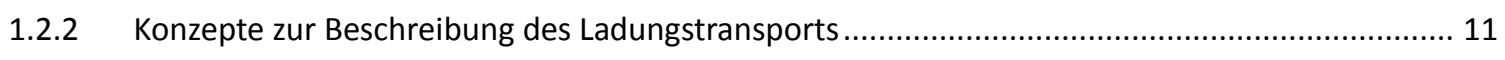

1.2.2.1 Diffuser Transport und delokalisierte Streuung: Das Drude-Sommerfeld-Modell................ 13

1.2.2.2 Ballistischer Transport und lokalisierte Streuung .......................................................... 15

1.2.2.3 Charakteristische Längenskalen des Ladungstransports................................................... 20

1.2.3 Bestimmung lokaler elektrochemischer Potentiale mit Hilfe der Raster-Tunnel-Potentiometrie. 21

1.2.4 Experimentelles Auflösungsvermögen ................................................................................... 26

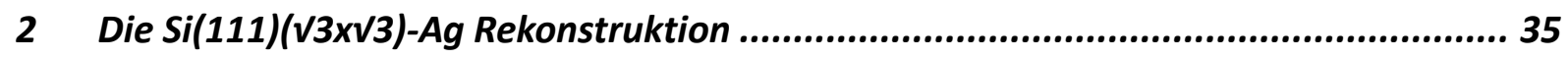

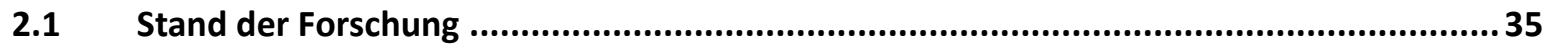

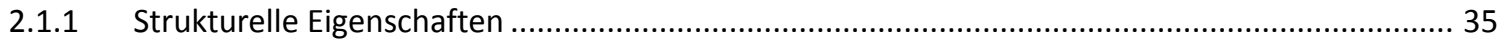

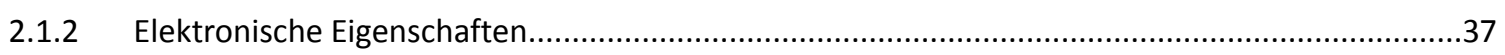

2.2 Präparation der Si(111)(V3xV3)-Ag Rekonstruktion ............................................. 42

2.3 Detaillierte Charakterisierung der verwendeten Proben ...........................................47

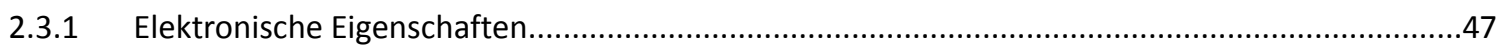

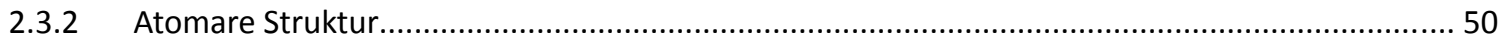

2.3.3 Silber-Atome als Donatoren auf der Si(111)(V3xV3)-Ag Rekonstruktion.................................. 53

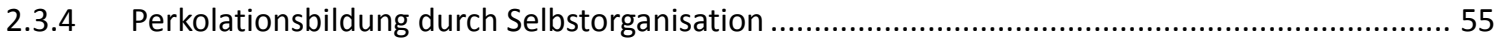

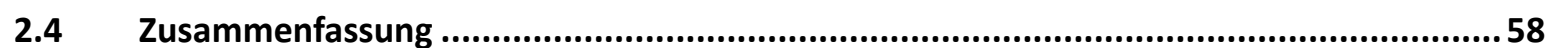


3 Ladungstransport durch die Si(111)(V3xV3)-Ag Rekonstruktion.............................. 59

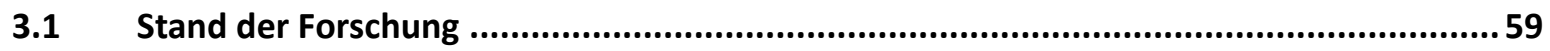

3.1.1 Die elektrische Leitfähigkeit der Si(111)(V3xV3)-Ag Rekonstruktion .......................................... 59

3.1.2 Kenntnisstand zu den mikroskopischen Transporteigenschaften ............................................... 63

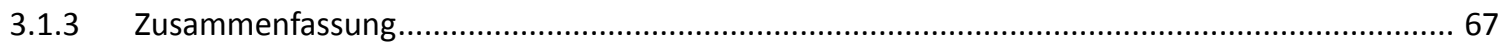

3.2 Raster-Tunnel-Potentiometrie auf dem natürlichen Perkolationsnetzwerk der

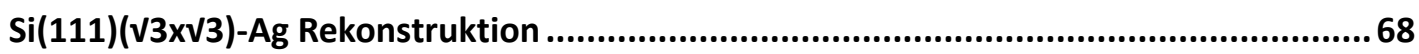

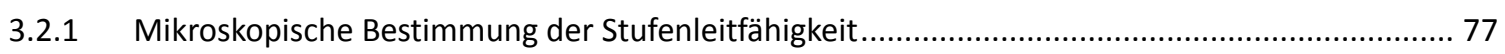

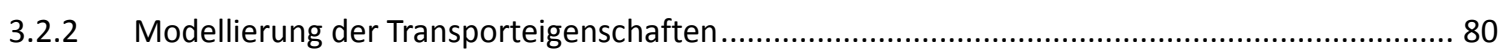

3.2.2.1 Ein analytisches Verfahren zur Lösung großer Widerstandsnetzwerke ............................... 81

3.2.2.2 Ergebnisse der Modellierung der Transporteigenschaften .................................................. 85

3.2.2.3 Von den mikroskopischen Transporteigenschaften zur makroskopischen Leitfähigkeit...... 92

3.3 Raster-Tunnel-Potentiometrie auf der Si(111)(V3xv3)-Ag Rekonstruktion auf stark gestuften Silizium Substraten ............................................................................97

3.4 Diskussion der mikroskopischen Transporteigenschaften ........................................ 104

4 Das elektrochemische Potential in der Umgebung von lokalisierten Defekten ..........105

4.1 Simulation der STP-Daten über einer Stufenkante ................................................... 112

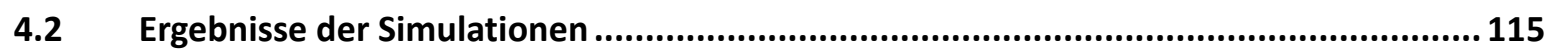

4.3 Interpretation der Variation des elektrochemischen Potentials in der Umgebung von

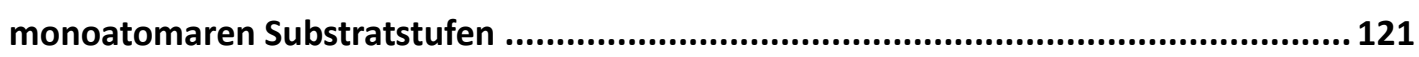

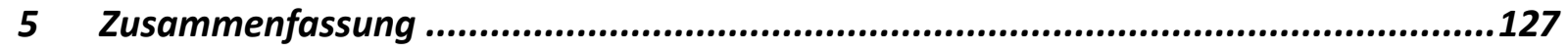

Anhang A: Konsistenzuntersuchungen zum Einfluss zusätzlicher Stützstellen ..................129

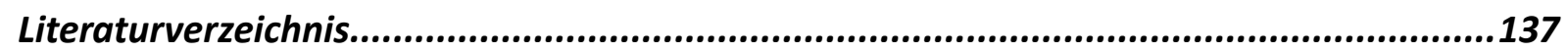

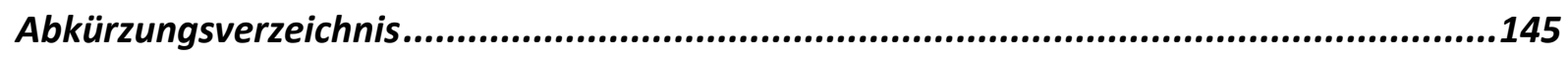

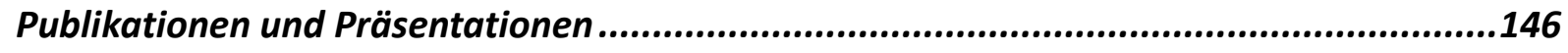

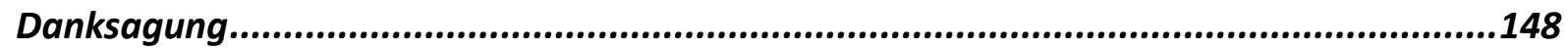

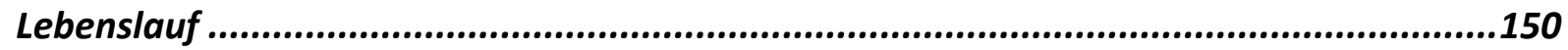




\section{Theoretische und experimentelle Grundlagen}

In diesem Abschnitt werden die theoretischen und experimentellen Grundlagen der vorliegenden Arbeit eingeführt. Zunächst wird eine theoretische Beschreibung der RasterTunnel-Mikroskopie (engl. Scanning Tunneling Microscopy, STM) vorgestellt, um anschließend den Messprozess der Raster-Tunnel-Potentiometrie zu diskutieren. Der Abschnitt Raster-Tunnel-Potentiometrie schließt mit einer Abhandlung zum theoretischen und experimentellen Auflösungsvermögen der verwendeten Anlage.

\subsection{Basistheorie der Raster-Tunnel-Mikroskopie}

Für die Interpretation von STM-Messungen ist eine theoretische Beschreibung des Tunnelstromes $\mathrm{I}_{\mathrm{T}}$ zwischen Spitze und Probe nötig. Hierfür wird die Besetzungsfunktion für Elektronen in Festkörpern benötigt, welche im thermischen Gleichgewicht durch die Fermifunktion $\mathrm{f}(\varepsilon, \mathrm{T})$ beschrieben wird [23]

$$
f(\varepsilon, T)=\left[1+\exp \left(\frac{\varepsilon}{k_{B} T}\right)\right]^{-1}
$$

wobei $\varepsilon$ die Energiedifferenz des Elektrons zum elektrochemischen Potential $\mu_{\mathrm{ec}}$ und $\mathrm{T}$ die Temperatur angibt. Das elektrochemische Potential $\mu_{\mathrm{ec}}$ ist im thermischen Gleichgewicht definiert als $\mu_{e c}=\mu_{c}-e \phi$, wobei $\mu_{\mathrm{c}}$ das chemische Potential und $\Phi$ das elektrische Potential für ein Elektron darstellt. Üblicherweise werden Spitze wie auch Probe eines STMs als voneinander unabhängige Elektronenreservoire im thermischen Gleichgewicht behandelt, zwischen denen Elektronentransport über eine Tunnelbarriere möglich ist. ${ }^{2}$ Wird eine elektrische Spannung $U_{\text {Bias }}$ angelegt, so verschieben sich die elektrochemischen Potentiale von

${ }^{2}$ Strenggenommen stört der Tunnelkontakt das thermische Gleichgewicht beider Subsysteme, da über die Tunnelbarriere Ladungsträger in das jeweils andere System injiziert werden. Für Au kann der relative Anteil injizierter „Stör“-Elektronen zu einer Größenordnung von $10^{-8}$ abgeschätzt werden, so dass die Störung durch den Tunnelprozess vernachlässigbar gering ist [30]. 
Spitze und Probe um einen Betrag e. $U_{\text {Bias }}$ gegeneinander. ${ }^{3}$ Stellt $\varepsilon$ die Energiedifferenz zum elektrochemischen Potential der Probe dar, so wird die Besetzungsfunktion der Probe durch $f\left(\varepsilon, T_{P}\right)$ und die der Spitze durch $f\left(\varepsilon+e U_{\text {Bias }}, T_{S}\right)$ angegeben. $\mathrm{T}_{\mathrm{P}}$ und $\mathrm{T}_{\mathrm{S}}$ bezeichnen jeweils die Temperatur von Probe und Spitze, welche unterschiedlich sein können. Eine weitere grundlegende Größe in der theoretischen Behandlung des Tunnelstromes ist die lokale Zustandsdichte $\rho_{P}(\vec{r}, \varepsilon)$ (engl. Local Density Of States, LDOS) der Probe. Multipliziert mit der Besetzungsfunktion gibt die LDOS an, mit welcher Wahrscheinlichkeit man am Ort $\vec{r}$ ein Elektron mit der Energie $\varepsilon$ findet. Die LDOS $\rho_{P}(\vec{r}, \varepsilon)$ ist also die Summe der Betragsquadrate aller Wellenfunktionen $\psi_{v}(\vec{r})$ mit Energie $\varepsilon_{v}=\varepsilon$ am Ort $\vec{r}$ :

$$
\rho_{\mathrm{P}}(\overrightarrow{\mathrm{r}}, \varepsilon)=\sum_{v}\left|\psi_{v}(\overrightarrow{\mathrm{r}})\right|^{2} \cdot \delta\left(\varepsilon_{v}-\varepsilon\right)
$$

Die Summation läuft hierbei über alle Quantenzahlen $v$ und die $\delta$-Funktion stellt sicher, dass nur Zustände mit einer Eigenenergie $\varepsilon_{v}=\varepsilon$ in die LDOS eingehen. Die Grundlagen zur Berechnung des Tunnelstrom zwischen zwei Elektroden gehen auf J. Bardeen zurück und darauf aufbauend entwickelten J. Tersoff und D. R. Hamann eine theoretische Beschreibung der Raster-Tunnel-Mikroskopie [24-29]. Die Tunnelspitze wird als s-artige Kugelwelle angenommen, welche um die Position $\vec{r}$ des äußersten Atoms der Spitze zentriert ist. Senkrecht zur Probenoberfläche wird ein exponentielles Abklingen der LDOS angenommen. Es kann gezeigt werden, dass der Tunnelstrom in diesem Fall eine relativ einfache Form annimmt [26-30]:

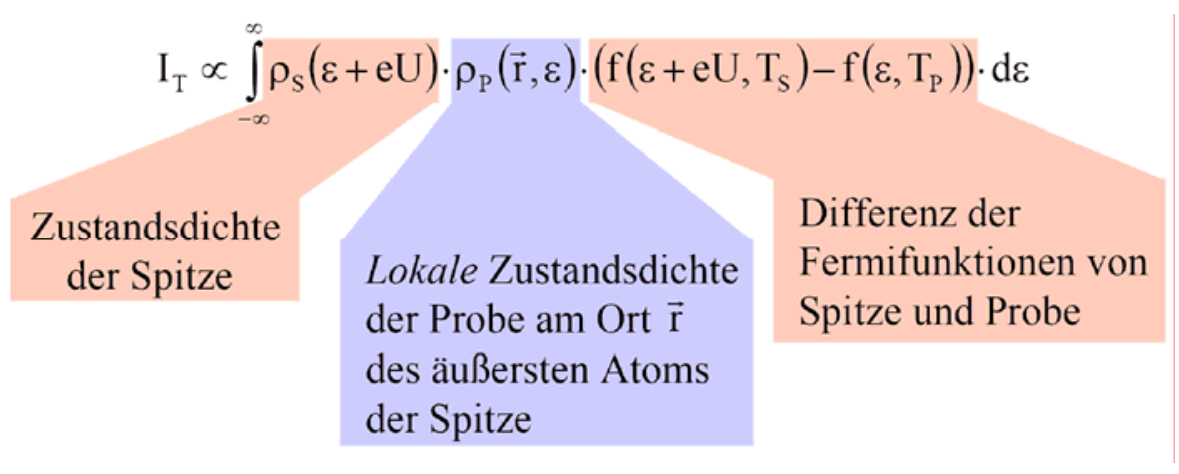

\footnotetext{
${ }^{3}$ Mit $U_{\text {Bias }}$ wird im Verlauf dieser Arbeit die elektrische Spannung gemäß $U_{\text {Bias }}=U_{\text {Spitze }}-U_{\text {Probe }}$ bezeichnet.
} 
Ist die Spitzenzustandsdichte im relevanten Energieintervall um $\varepsilon+e U_{\text {Bias }}$ konstant, bildet der Tunnelstrom direkt die lokale Zustandsdichte der Probe am Ort der Spitze ab. N. D. Lang formuliert die Summation über die Zustände in ein Integral über die Energie um und beschreibt den Tunnelstrom über die LDOS bei $\mathrm{z}=0$, d. h. der LDOS am Ort der Probe. Die LDOS der Probe klingt bei dieser Modellierung exponentiell ins Vakuum ab und der Tunnelstrom ergibt sich zu

$$
I_{T}(x, y, z) \propto \int_{E_{f}}^{E_{f}+e U_{\text {Bias }}}\left[\operatorname{LDOS}(x, y, z=0, E) e^{-2 z \sqrt{2(W-E)+e U_{\text {Bias }}}}\right] d E,
$$

mit $\mathrm{z}$ der Spitzenhöhe und $\mathrm{W}=\Phi+\mathrm{E}_{\mathrm{f}}$ der Barrierenhöhe, wobei $\Phi$ die mittlere Austrittsarbeit von Probe und Spitze ist [31]. Angelehnt an diese Beschreibung wird in Kapitel 4 eine Simulation zur Interpretation von Raster-Tunnel-Potentiometrie Daten entwickelt.

\subsubsection{Raster-Tunnel-Topographie}

Bei dem im Rahmen dieser Arbeit verwendeten Topographiemodus wird eine konstante Spannung $U_{\text {Bias }}$ zwischen Spitze und Probe angelegt. Während der Rasterung der Spitze in Xund y-Richtung wird der Tunnelstrom $\mathrm{I}_{\mathrm{T}}$ erfasst und auf einen Sollwert durch Verändern der Spitzenhöhe geregelt (Modus: „,constant-current“). Bei Unterschreitung des Sollwertes wird die Spitze in z-Richtung näher an die Probe herangeführt und bei Überschreitung entsprechend zurückgezogen. An jedem Punkt $(\mathrm{x}, \mathrm{y})$ wird für $\mathrm{I}_{\mathrm{T}}=\mathrm{I}_{\text {soll }}$ die Auslenkung $\mathrm{z}$ des Piezostellelementes erfasst, so dass die „Topographie“ als eine Fläche konstanten Tunnelstromes für eine festgehaltene Spannung verstanden wird. Zur Veranschaulichung soll ein Beispiel gezeigt werden, bei dem die Zustandsdichte der Spitze als konstant angenommen wird und die Näherung $\left|\mathrm{eU}_{\text {Bias }}\right| » \mathrm{k}_{\mathrm{B}} \mathrm{T}$ gilt. Das Tersoff-Hamann Integral vereinfacht sich dann $\quad \mathrm{zu} \quad \mathrm{I}_{\mathrm{T}} \propto \int_{0}^{\mathrm{eU}} \rho_{\mathrm{P}}(\overrightarrow{\mathrm{r}}, \varepsilon) \cdot \mathrm{d} \varepsilon$ und die Spitze folgt für $\mathrm{I}_{\mathrm{T}}=\mathrm{I}_{\text {soll }}$ einer Fläche konstanter integrierter lokaler Zustandsdichte im Energiebereich zwischen $\varepsilon=0$ und $\varepsilon=e U_{B i a s}$. 


\subsubsection{Raster-Tunnel-Spektroskopie}

Bei der dI/dU-Spektroskopie wird an einer festgehaltenen Spitzen-Position die Tunnelspannung U variiert und die Änderung des Stroms dI gemessen. Für kleine Spannungsvariationen $\mathrm{dU}$ und bei einer als konstant angenommenen Zustandsdichte der Spitze kann gezeigt werden, dass

$$
\left.\frac{\mathrm{dI}}{\mathrm{dU}} \propto \rho_{\mathrm{P}}(\overrightarrow{\mathrm{r}}, \varepsilon)\right|_{\varepsilon=\mathrm{eU}}[25]
$$

Die Raster-Tunnel-Spektroskopie erlaubt somit einen direkten Zugang $\mathrm{zu}$ der lokalen Zustandsdichte der Probe.

\subsection{Raster-Tunnel-Potentiometrie}

\subsubsection{Experimenteller Aufbau}

Abbildung 2 (a) zeigt ein makroskopisches Pendant zur Raster-Tunnel-Potentiometrie. Ein Widerstand wird von einem Strom $\mathrm{I}_{\text {macro }}$ durchflossen. Ein variabler Schleifkontakt greift innerhalb des Widerstands die elektrische Spannung $U_{\text {lok }} a b$, welche stromlos über eine Kompensationsspannung gemessen wird. Als Funktion der Schleifposition variiert die elektrische Spannung $\mathrm{U}_{\mathrm{lok}}(\mathrm{x})$. Die elektrische Spannung kann z. B. farbkodiert als Funktion des Ortes aufgetragen werden.

Bei der Raster-Tunnel-Potentiometrie wird prinzipiell dasselbe Verfahren angewendet:

1. Eine elektrisch leitfähige Probe, wie z. B. das zweidimensionale Elektronengas der $\operatorname{Si}(111)(\sqrt{3} \mathrm{x} \sqrt{3})$-Ag Rekonstruktion, wird im UHV kontaktiert, um einen elektrischen Strom aufzuprägen.

2. Das Tunnelmikroskop wird verwendet, um in einem ersten Schritt die strukturellen Eigenschaften der Probe im Topographiemodus zu analysieren. Eine entsprechende Topographie zeigt die 3D-Darstellung aus Abbildung 2 (b).

3. Für jeden „topographischen“ Messpunkt kann zudem das elektrochemische Potential der Probe bestimmt werden. Die experimentelle Umsetzung der PotentiometrieMessung geschieht ebenfalls als Kompensationsmethode. Bei festgehaltener Spitzenposition wird durch einen zweiten Regelkreis für jeden Rasterpunkt das elektrochemi- 
sche Potential der Spitze $\mu_{\mathrm{ec}, \text { Spitze }}$ derart so eingestellt, dass im zeitlichen Mittel kein Tunnelstrom $I_{t}$ fließt. Im selben Sinn wie (a) entspricht das Spitzenpotential damit dem lokalen Potential am Ort des Tunnelkontakts. Mit noch zu diskutierenden Details folgt das Spitzenpotential mit dieser Messvorschrift dem lokalen elektrochemischen Potential der Probe $\mu_{e c, \text { Spitze }}(x, y)=\mu_{e c, \text { Probe }}(x, y) .{ }^{4}$ Eine gemessene Potentiallandschaft ist farbkodiert der 3D-Topographie in Abbildung 2 (b) überlagert.

a)

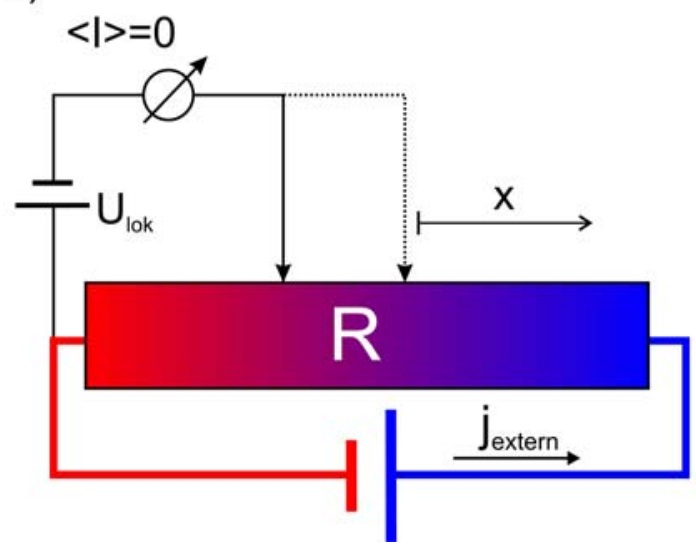

b)

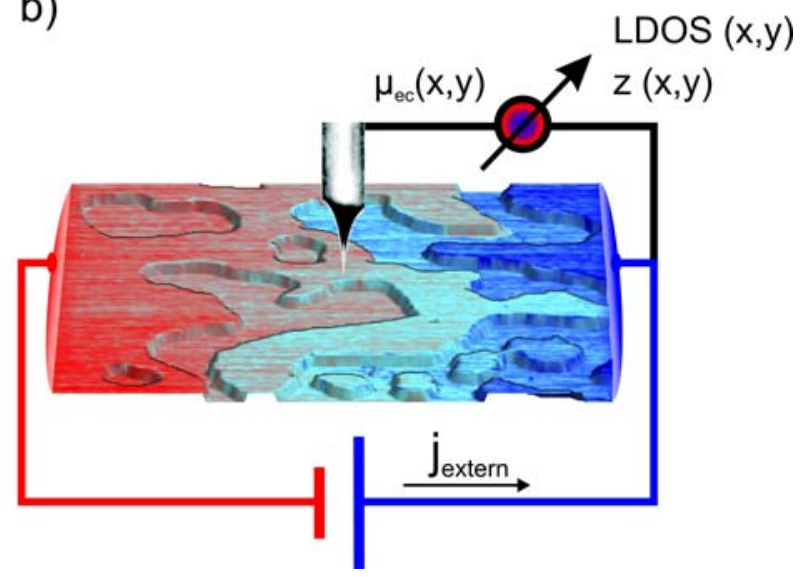

Abbildung 2: (a) zeigt ein makroskopisches Pendant zur Raster-Tunnel-Potentiometrie. (b) zeigt schematisch das Verfahren der Raster-Tunnel-Potentiometrie. Die Tunnelspitze wird benutzt, um sowohl die strukturellen Informationen der Probe als auch das lokale Probenpotential zu vermessen. Die 3D-Darstellung zeigt $400 \times 400 \mathrm{~nm}^{2}$ der gemessenen Topographie der Si(111) $(\sqrt{ } 3 x \sqrt{ } 3)$-Ag Rekonstruktion. Die gemessene Potentiallandschaft ist farbkodiert dargestellt. Das Potential variiert um $1 \mathrm{mV}$ von blau nach rot.

Dieses Verfahren wurde von M. A. Schneider 1993 in Göttingen entwickelt und im Rahmen meiner Diplomarbeit durch eine digitale Ansteuerung und eine 6-fache in situ UHVKontaktierung der Probe weiterentwickelt [32, 33]. Ein bestehendes Tieftemperatur-STM wurde gemäß diesen Weiterentwicklungen für STP-Messungen umgebaut [34, 35].

\footnotetext{
${ }^{4}$ Eine Diskussion dieser Größe wird aus Übersichtsgründen im nächsten Kapitel vorgenommen. Für diesen Abschnitt genügt es, sich unter dem elektrochemischen Potential eine „elektrische Spannung“" vorzustellen.
} 
Ein Blockdiagramm der digitalen Ansteuerung illustriert Abbildung 3. Der laterale Querstrom $\mathrm{j}_{\text {extern }}$ durch die Probe wird über zwei 16-Bit Digital-Analog-Wandler (engl. DAC) mit anschließender Leistungsverstärkung aufgeprägt. ${ }^{5}$ Durch affines Verändern der elektrischen Spannungen an beiden Probenenden kann bei gleichem lateralem Querstrom das elektrochemische Potential der Probe am Ort der Tunnelspitze im Vergleich zum Erdpotential verändert werden. Während der Topographie-Messung werden die Spannungen an beiden Probenenden bei konstanter Differenz derart justiert, dass das elektrochemische Potential am Ort des Tunnelkontakts der gewünschten Bias-Spannung entspricht. Bei der PotentiometrieRegelphase hingegen werden die Spannungen an beiden Probenenden bei konstanter Differenz um die Bias-Spannung vermindert und es erfolgt ein Nullabgleich des Tunnelstroms. Ein weiterer 16-Bit-DAC ( $\left.U_{\text {Spitze }}\right)$ variiert dazu über den nicht-invertierenden Eingang des Tunnelstromverstärkers das elektrochemische Potential der Spitze im $\mu V$-Bereich. Das elektrochemische Potential der Tunnelspitze folgt auf diese Weise, mit noch zu diskutierenden Details, dem lokalen elektrochemischen Potential der Probe am Ort des Tunnelkontakts. Ist im weiteren Verlauf dieser Arbeit von dem elektrochemischen Potential der Probe die Rede, so ist damit stets die Kompensationsspannung am DAC $U_{\text {Spitze }}$ gemeint, die für eine Nullkompensation des Tunnelstroms notwendig ist. Eine detailliertere Beschreibung der Ansteuerungselektronik ist z. B. in Ref. [33, 36] zu finden.

\footnotetext{
${ }^{5}$ Um das Rauschen sowie die Bit-Auflösung zu optimieren, kann die Spannung beider Ausgänge über einen Widerstandsteiler herunter geteilt werden. Hierfür werden Präzessionswiderstände $\left(\Delta \mathrm{R}_{60^{\circ} \mathrm{C}} / \mathrm{R}_{20^{\circ} \mathrm{C}}\right.$ max. $\left.\pm 0.5 \%\right)$ mit einer maximalen Belastung von je $50 \mathrm{~W}$ verwendet.
} 


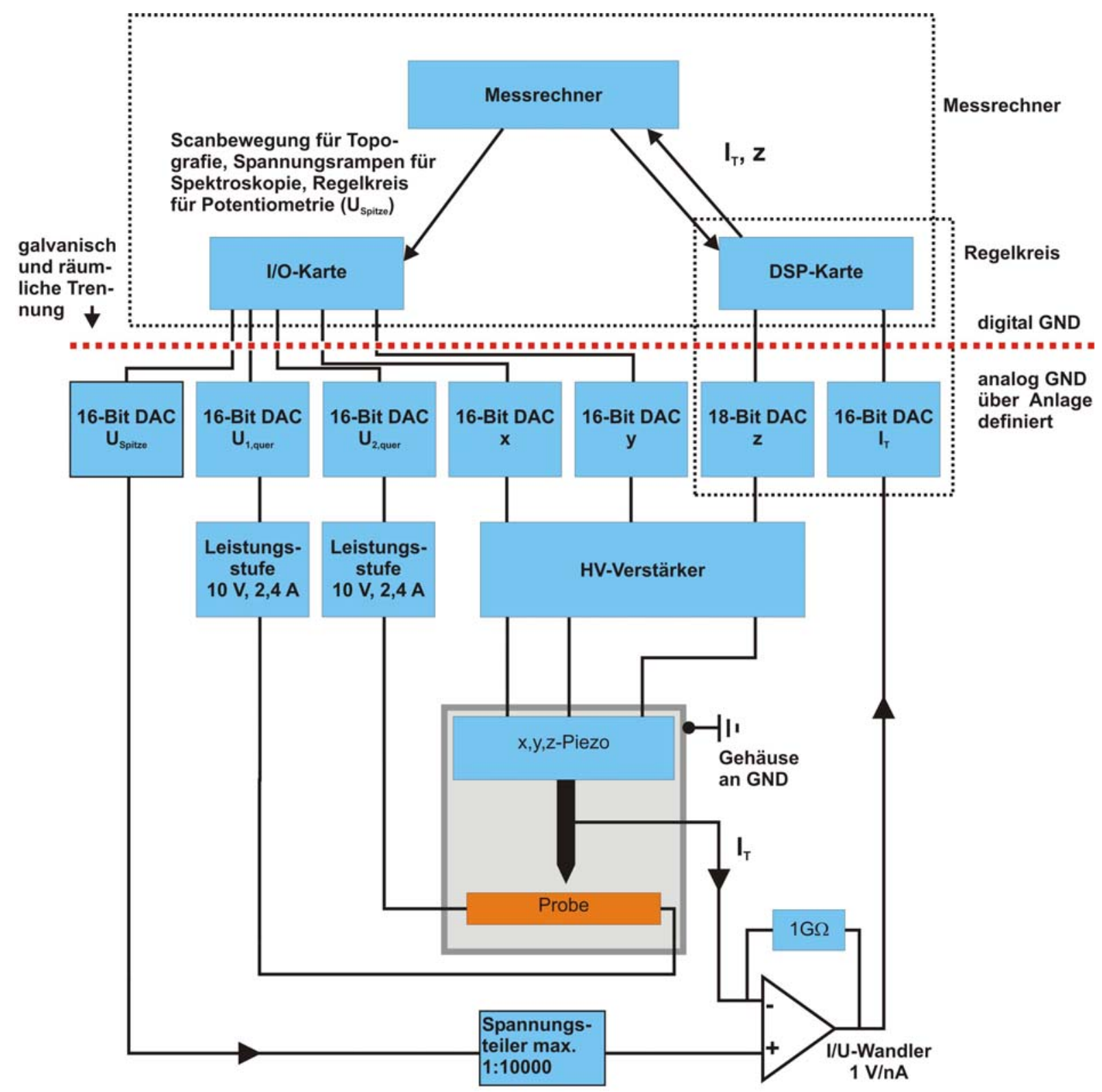

Abbildung 3: Blockdiagramm der Messelektronik zur Raster-Tunnel-Potentiometrie. Abbildung aus Ref. [36] gemäß Diplomarbeit [33].

Eine Fotografie des STM-Kopfs für STP-Messungen bei Raumtemperatur zeigt Abbildung 4. Vier der sechs möglichen Kontaktsegmente des Probenschlittens sind sichtbar. Exemplarisch ist eine Probe mit aufgedampften Gold-Stegen, welche durch Drähte mittels „Bonding“ kontaktiert worden sind, gezeigt. Für eine ausführliche Beschreibung der übrigen STMKomponenten wird auf Ref. [37, 38] verwiesen. 


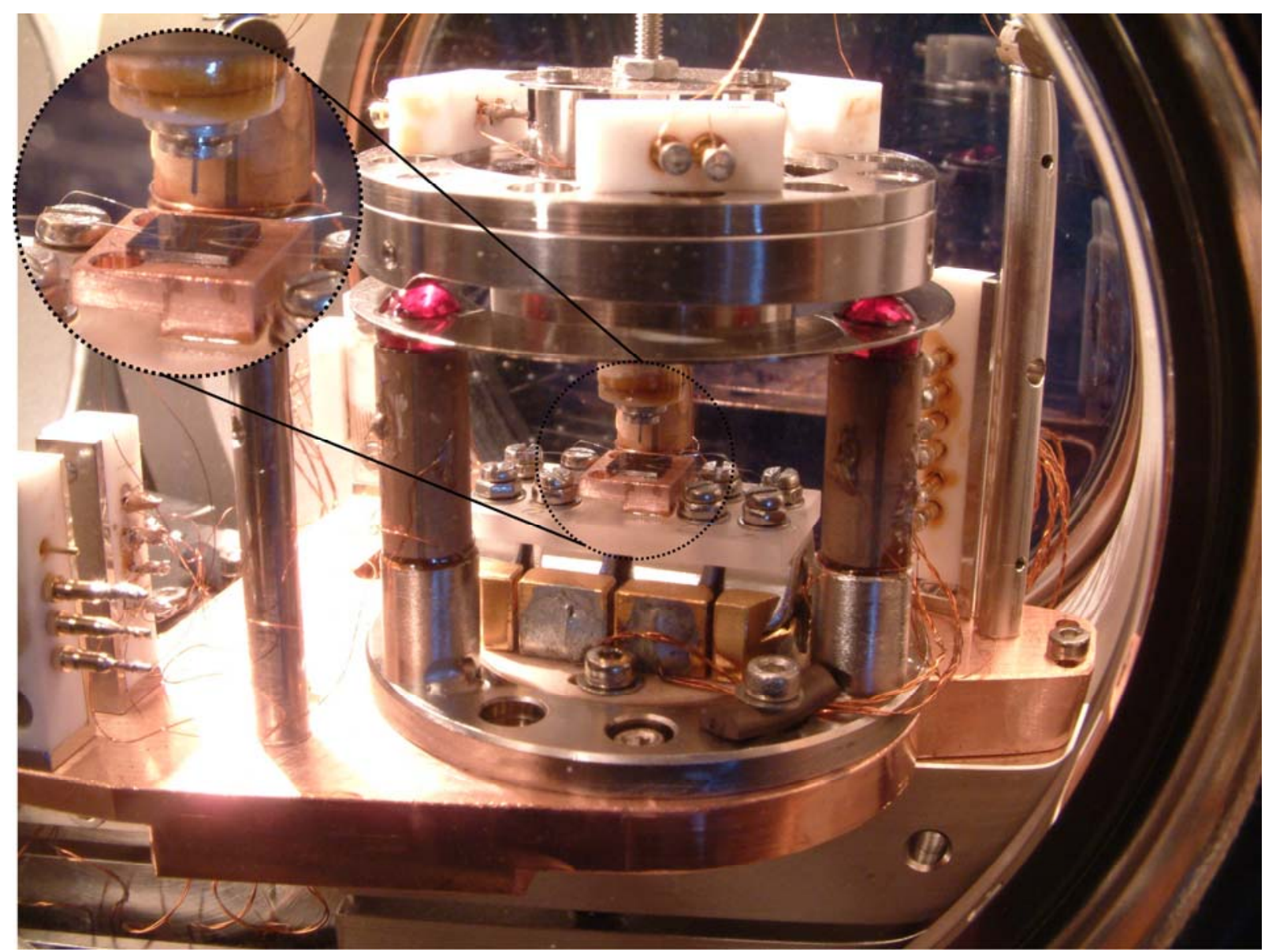

Abbildung 4: Fotografie des umgebauten STM's. Eine Probe mit aufgedampften Au-Stegen wurde mit $33 \mu \mathrm{m}$ dicken Al-Drähten durch „Bonding“ kontaktiert. Zu erkennen sind vier der sechs voneinander elektrisch isolierten Kontaktmöglichkeiten des Probenschlittens. 


\subsubsection{Konzepte zur Beschreibung des Ladungstransports}

Unterschiedliche Konzepte werden zur Beschreibung des Ladungstransports benutzt. Allen Konzepten gemeinsam ist die Tatsache, dass der Ladungstransport durch eine NichtGleichgewichtsverteilung der Besetzungsfunktion zustande kommt.

In homogenen Systemen gelingt eine makroskopische Beschreibung, indem der Ladungstransport im k-Raum behandelt wird. In diesem Rahmen werden thermodynamische Gleichgewichtsgrößen durch z. B. im k-Raum verschobene Besetzungsfunktionen $f\left(\vec{k}+\vec{k}_{d}\right)$ und das elektrochemische Potential $\mu_{e c}=\mu_{c}-e \phi$ definiert. Insbesondere $\mu_{\mathrm{ec}}$ ist hierbei eine zentrale Größe, die bei diffusem Ladungstransport, abgesehen vom Vorzeichen, den gleichen räumlichen Verlauf wie das elektrostatische Potential $\phi$ hat. Die lokale Ladungsdichte ist gemäß der Poisson-Gleichung $\Delta \phi(\vec{r})=-\frac{\rho(r)}{\varepsilon_{0}}$ dabei selbstkonsistent mit dem elektrostatischen Potential verknüpft.

Die Herausforderung besteht in der Modellierung des Ladungstransports auf der mikroskopischen Skala. Auf Längenskalen kürzer als die Energierelaxationslänge und die Phasenkohärenzlänge $l_{\phi}$ kann nicht mehr von thermodynamischen Besetzungsfunktionen Gebrauch gemacht werden. Sowohl die lokalisierte Streuung an Defekten als auch delokalisierte Prozesse (wie z.B. Elektron-Elektron- und Elektron-Phonon-Wechselwirkung) müssen unter Berücksichtigung der inhomogenen Nicht-Gleichgewichtsverteilung vollständig quantenmechanisch auf gleicher Ebene (lokal und global) behandelt werden. Ebenso können die lokalen Besetzungsfunktionen nicht mehr trivial über die Kopplung an ein thermisches Bad definiert werden, sondern ergeben sich dynamisch aus sämtlichen Prozessen. Dieser Sachverhalt erschwert eine geschlossene Beschreibung bis heute, so dass häufig die elektrostatische Abschirmung als ein Prozess der Elektron-Elektron-Wechselwirkung vernachlässigt werden muss und nicht selbstkonsistent behandelt wird. . So modelliert bspw. S. Datta die Abschirmung entsprechend einer Gleichgewichtssituation, so dass das elektrochemische Potential am Ort der Barriere „almost discontinuously“ auf kürzester Längenskala variiert (Ref. [39], Seite 73). 
Um den Ladungstransport einheitlich analysieren und eventuell konzeptionell beschreiben zu können, sind Informationen über $\phi(\vec{r}), \rho(\vec{r})$, sowie die Besetzungsfunktionen mit atomarer Auflösung im Nicht-Gleichgewicht nötig, wofür bislang keine experimentellen Techniken verfügbar sind.

Im Folgenden wird die Raster-Tunnel-Potentiometrie als eine experimentelle Technik vorgestellt, die ein „Abbild“ des lokalen elektrochemischen Potentials auf atomarer Skala im Nicht-Gleichgewicht liefern kann. Die neben der lokalen Zustandsdichte gewonnene Messgröße stellt ein Maß für die über die Energie gemittelte Verteilungsfunktion des NichtGleichgewichts dar. Da am Ort der Probe keine thermischen Besetzungen vorliegen müssen, wird die Messgröße kritisch diskutiert und als Abgrenzung zum elektrochemischen Potential des Gleichgewichts durch $\mu_{\mathrm{ec}, \mathrm{STP}}$ bezeichnet.

Im Rahmen der Beschreibung des diffusen Ladungstransports nach Drude-Sommerfeld werden zunächst die wichtigsten Begriffe eingeführt, um anschließend den Grenzfall des ballistischen Ladungstransports zu diskutieren. Dieser Abschnitt schließt mit einer Diskussion zu den charakteristischen Längenskalen des Ladungstransports.

Grenzen dieser Beschreibung werden an zwei Stellen in dieser Arbeit diskutiert. Zum einen wird der Messprozess der Raster-Tunnel-Potentiometrie kritisch betrachtet (Kapitel 1.2.3), zum anderen zeigen lateral hoch aufgelöste Messungen von $\mu_{\mathrm{ec}, \mathrm{STP}}$ Abweichungen der aktuellen Modellvorstellung nach S. Datta [39]. Diese Daten werden im Kapitel 4 vorgestellt und ausführlich diskutiert. 


\subsubsection{Diffuser Transport und delokalisierte Streuung: Das Drude- Sommerfeld-Modell}

Das Drude-Sommerfeld-Modell des diffusen Ladungstransports wurde im Jahre 1900 von Paul Drude entwickelt und 1933 von A. Sommerfeld und H. Bethe vervollständigt. Bewegen sich $\mathrm{n}$ Elektronen pro Einheitsvolumen mit einer Geschwindigkeit $\vec{v}$, so ist die Stromdichte als $\vec{j}=-n \overrightarrow{e v}$ definiert, da jedes Elektron eine Ladung von -e trägt. Betrachtet man ein ruhendes Elektronengas im Gleichgewicht, dann verschwindet der Zeit und Ensemble Mittelwert aller Geschwindigkeiten und die resultierende Stromdichte ist null. Liegt jedoch ein konstantes elektrisches Feld an, so werden alle Elektronen gemäß $F=m^{*} d v / d t=-e E$ beschleunigt. Durch diffuse Stoßprozesse kehren die Elektronen nach der mittleren Streuzeit $\tau$ ins Gleichgewicht zurück und werden im elektrischen Feld erneut beschleunigt. Der Streuprozess führt die im elektrischen Feld hinzugewonnene kinetische Energie an eine Senke ab (z. B. an das Phononenbad). Im zeitlichen und Ensemble Mittel bewegen sich alle Elektronen mit der Driftgeschwindigkeit $v_{D}=-e \tau / m^{*} \cdot E$, welche mit der FermiGeschwindigkeit $v_{f}=\sqrt{2 E_{f} / m^{*}}$ durch die mittlere freie Weglänge $l_{M F P}$ ausgedrückt werden kann zu $v_{D}=\frac{-e l_{M F P}}{m^{*} v_{f}} \cdot E .^{6}$ Mit der Definition der Stromdichte folgt hieraus das ohmsche Gesetz im Rahmen des Drude-Sommerfeld-Modells zu

$$
j=\sigma E \text {, mit } \sigma=\frac{n e^{2} l_{M F P}}{m^{*} v_{f}}=\frac{n e^{2}}{m^{*}} \tau[23,40] .
$$

\footnotetext{
${ }^{6}$ Auf dem für diese Arbeit wichtigen freien Elektronengas der $\operatorname{Si}(111)(\sqrt{3} x \sqrt{3})$-Ag Rekonstruktion beträgt $l_{M F P}$, entsprechend den experimentellen Ergebnissen aus Kapitel 3.2.2.2, etwa $70 \mathrm{~nm}$.
} 
Die Besetzungsfunktion $f(\vec{k})$ entspricht einer verschobenen Fermikugel, welche für eine Dimension schematisch in Abbildung 5 dargestellt ist. Um einem Netto-Elektronentransport von links nach rechts zu entsprechen, ist die Gesamtbesetzung der $\mathrm{k}^{+}$-Zustände größer als die der k-Zustände. Die nach der Poisson-Gleichung zum elektrostatischen Potential korrespondierenden Ladungsdichten befinden sich in den Kontakten (nicht gezeigt) und innerhalb des Leiters entspricht die Ladungsdichte die des ungestörten Systems.

Das chemische Potential kann bei dieser Art der Beschreibung in k-abhängige, quasichemische Potentiale aufgespalten werden [23, 39, 40]. Es wird postuliert, dass sich jedes quasi-chemische Potential in einem stationären Gleichgewicht befindet und sich deshalb trotz der vorherrschenden Nicht-Gleichgewichtsverteilung durch thermodynamische Zustandsvariablen beschreiben lässt. Die quasi-chemischen Potentiale sind ortsunabhängig, da die Positionen innerhalb des Leiters ununterscheidbar sind oder anders herum argumentiert, es keine lokalisierten Defekte gibt. Das elektrochemische Potential folgt damit dem Verlauf des elektrostatischen Potentials $\mu_{e c}(x, y)=-e \phi(x, y)+$ const. und die Energiedissipation findet ortsunabhängig auf der gesamten Länge des Leiters statt.

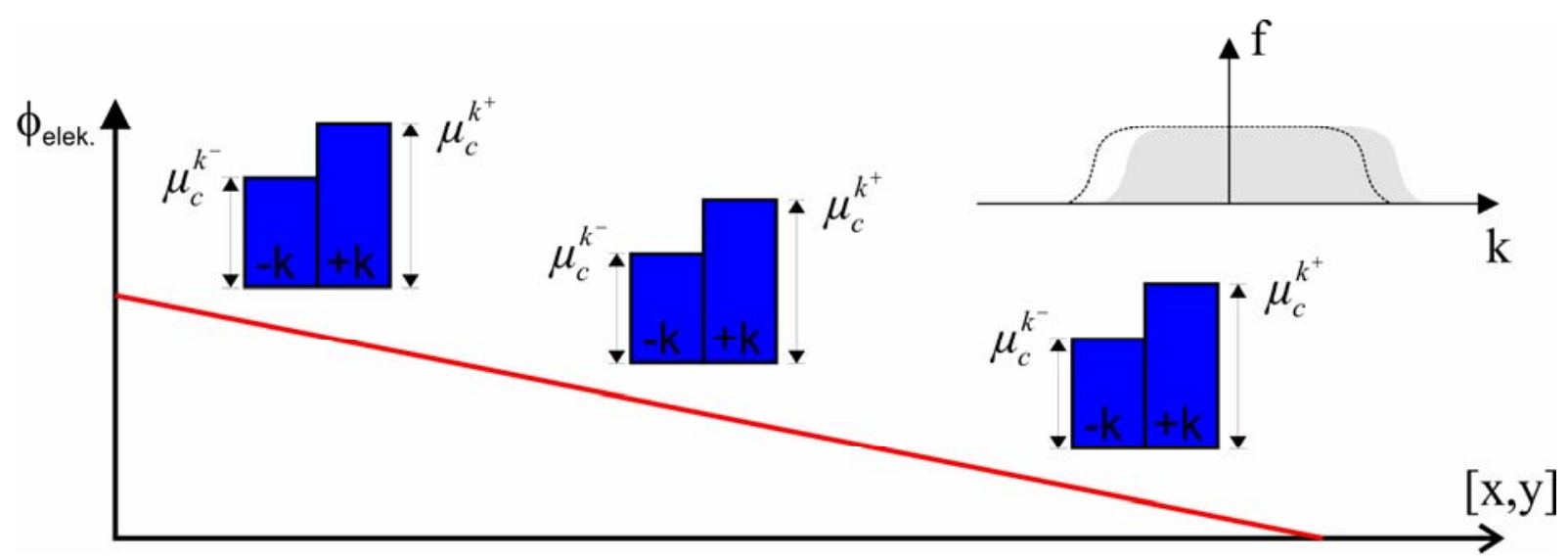

Abbildung 5: Schemaskizze zum Verlauf des elektrostatischen und des chemischen Potentials innerhalb eines Leiters mit ausschließlich diffuser Streuung. Die quasi-chemischen Potentiale sind ortsunabhängig und das elektrochemische Potential folgt dem elektrostatischen Potential $\mu_{e c}(x, y)=-e \phi(x, y)+$ const. . Der Inset zeigt ein Piktogramm der Besetzungsfunktion $f(k)$. 


\subsubsection{Ballistischer Transport und lokalisierte Streuung}

Für einen großen, z. B. zweidimensionalen Leiter verhält sich der elektrische Leitwert wie $G=\sigma \frac{b}{l}$. Groß bedeutet in diesem Zusammenhang eine Kombination aus Länge 1 und Breite b. Gemäß dieser Beschreibung müsste der elektrische Leitwert für beliebig kleine Leiterlängen divergieren. Experimentell wird jedoch eine endliche Leitfähigkeit beobachtet, wenn die Systemgrößen unterhalb der mittleren freien Weglänge $l_{M F P}$ sind [41]. Dies wird als ballistischer Transport bezeichnet und der Widerstand, gemessen an den Stromzuführungen, beträgt $G_{\text {System }}^{-1}=\frac{h}{2 e^{2} M}=\frac{12,9 k \Omega}{M}$ mit $M \in \mathbb{N}_{>0}[39,42]$. Die Zahl M bezeichnet die Anzahl von lateralen Quantisierungsmoden, welche unter Berücksichtigung periodischer Randbedingungen quer zur Transportrichtung zu $M=\operatorname{Integer}\left(\frac{2 b}{\lambda_{f}}\right)$ abgeschätzt werden kann [39]. Die elektrische Leistung verbunden mit diesem Widerstand wird nicht innerhalb des Leiters, sondern an den Kontakten dissipiert. Innerhalb des Leiters geschieht der Ladungstransport völlig verlustfrei. Die Besetzungsfunktion entspricht einer verschobenen Fermikugel und das elektrostatische Potential ist innerhalb des ballistischen Leiters konstant.

Eine Inhomogenität innerhalb des ballistischen Leiters verringert die „,ideale“ Leitfähigkeit. Die Ursache hierfür ist eine lokale Abweichung vom periodischen Potential des Kristalls, was eine Streuung der kristallperiodischen Bloch-Zustände zur Folge hat. Mikroskopisch betrachtet können dies Leerstellen oder Fremdatome bis hin zu ausgedehnten Defekten wie z. B. Versetzungen oder Korngrenzen im Kristall sein. Eine Modellierung geschieht meist in Form einer quantenmechanischen Tunnelbarriere, die Elektronen mit der Wahrscheinlichkeit $\mathrm{T}$ transmittieren können. 
Im Rahmen der von Landauer 1957 eingeführten Beschreibung ergibt sich der Widerstand eines ballistischen Systems mit einer Barriere der Transmission T zu

$$
G_{\text {System }}^{-1}=\frac{12,9 k \Omega}{M} T[39,42-45]^{7}
$$

Am Ort der Barriere kommt es zur Ausbildung eines Ladungsdipols, um den elektrostatischen Potentialunterschied zu beiden Seiten der Barriere abzuschirmen. Sowohl das elektrostatische Potential als auch die Ladungsdichte variieren am Ort der Barriere innerhalb der elektrostatischen Abschirmlänge. Der induzierte Dipol wird als Landauer-Widerstands-Dipol (engl. Residual-Resistivity-Dipol) bezeichnet und wurde als die mikroskopische Ursache für den defektinduzierten elektrischen Widerstand erkannt [39, 43-45].

Abbildung 6 zeigt eine Originalabbildung von S. Datta zum Verlauf der Potentiale am Ort der Barriere [39]. Das elektrostatische Potential $\Phi$ variiert wie im Gleichgewichtsfall innerhalb der elektrostatischen Abschirmlänge und es kommt zur Ausbildung eines Ladungsdipols. Das chemische Potential ist über die lokale Ladungsdichte gemäß der Poisson-Gleichung $\Delta \phi(\vec{r})=-\frac{\rho(r)}{\varepsilon_{0}}$ dabei selbstkonsistent mit dem elektrostatischen Potential verknüpft. Die Variation des elektrostatischen Potentials korrespondiert mit einer Variation im chemischen Potential $\mu_{\mathrm{c}}$ und das resultierende elektrochemische Potential $\mu_{e c}=\mu_{c}-e \phi$ ist bis zur Barriere konstant [39, 40]. Es verändert sich abrupt am Ort der Barriere und der Autor selbst bezeichnet die Variation als ,almost discontinuously“ (Ref. [39], Seite 73), obwohl in seinen Abbildungen ein nicht weiter erwähnter Übergangsbereich zu erkennen ist. Die elektrostatische Abschirmung wird wie für ein System im Gleichgewicht betrachtet, dass heißt ohne Transport.

\footnotetext{
${ }^{7}$ Im Rahmen dieser Beschreibung ist nur der elektrochemische Potentialunterschied entsprechend $G_{\text {Barriere }}^{-1}=\frac{12,9 \mathrm{k} \Omega}{M} \frac{1-T}{T}$ an der Barriere lokalisiert und der restliche elektro-
} chemische Potentialabfall findet an den Kontakten statt. 
(a)

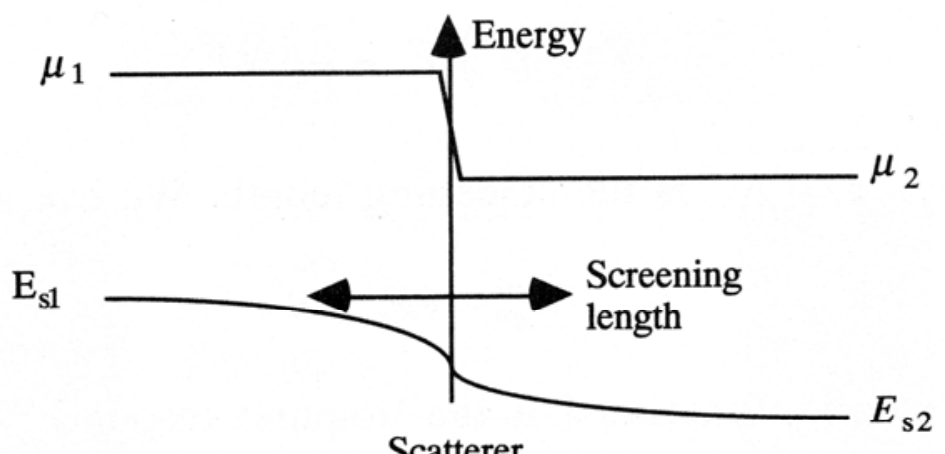

(b)

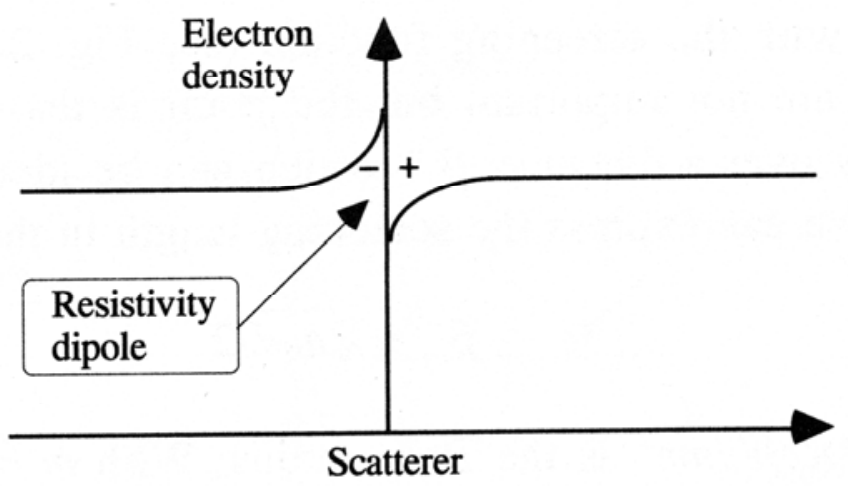

(c)

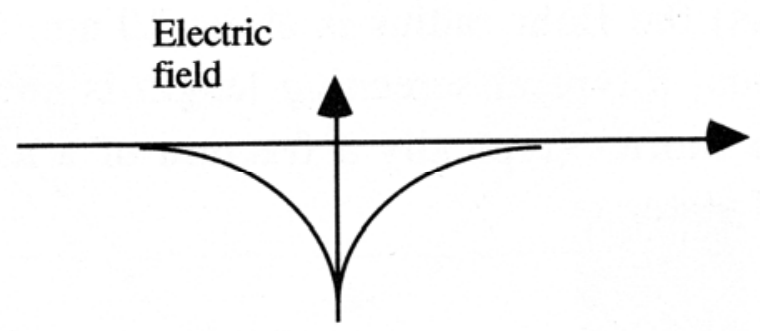

Fig. 2.3.3. Spatial variation of $(a)$ conduction band-edge and electrochemical potential, $(b)$ electron density and $(c)$ electric field across a scatterer.

Abbildung 6: Originalabbildung aus Ref. [39] zum Verlauf des elektrochemischen Potentials $(\mu)$ und des elektrostatischen Potentials $\left(E_{s}=-e \Phi\right)$ an einem Streuer in einem ballistischen Leiter. (b) zeigt den Ladungsdipol am Ort der Barriere, welcher das elektrostatische Potential zu beiden Seiten der Barriere innerhalb der elektrostatischen Abschirmlänge abschirmt. In (c) ist das daraus resultierende elektrische Feld gezeigt. 
W. Zwerger und K. Schönhammer berechneten den strominduzierten Dipol (LandauerWiderstands-Dipol) im Rahmen einer exakten quantenmechanischen Streutheorie für ein zweidimensionales Elektronengas, dessen Besetzung fern des Streuzentrums im Rahmen einer verschobenen Fermikugel modelliert wird [46]. Abbildung 7 zeigt die normalisierte Ladungsdichte an einer harten Scheibe als lokalisierten Defekt. Das Ergebnis erinnert an eine Friedel-Oszillation mit leichten Korrekturen. So kommt es durch die heran strömenden Ladungen zu einer Erhöhung der Elektronendichte vor der Barriere und einer korrespondierenden Verarmung dahinter, so dass die Ladungsdichte am Ort der Barriere in erster Ordnung der semiklassischen Beschreibung folgt [46]. ${ }^{8}$

Am Ort der Barriere transmittieren Elektronen aus Zuständen von der einen Seite der Barriere in freie Zustände auf der anderen Seite. Besitzt die Barriere keine internen Freiheitsgrade, so findet der Tunnelprozess elastisch statt. Die Dissipation gemäß der Joulschen Wärme I.G ist dann nicht in oder an der Barriere lokalisiert und die Besetzungsfunktionen (plural für die quasi-Niveaus) können sich am Ort der Barriere von der thermischen Besetzung unterscheiden. Innerhalb der Energie-Relaxationslänge werden die thermischen Besetzungsfunktionen wiederhergestellt und die assoziierten Differenzenergien werden an das Kristallgitter übergeben. ${ }^{9}$ Dasselbe gilt für die Kontaktbereiche und die damit assoziierten Kontaktwiderstände [39].

${ }^{8}$ Denselben Effekt kann man beispielsweise an Brückenpfeilern in strömenden Flüssen beobachten.

${ }^{9}$ Eine Thermalisierung innerhalb des elektronischen Systems kann durch Elektron-ElektronStreuung erfolgen. Die Differenzenergie zur Gittertemperatur wird durch Elektron-PhononStreuung an das Kristallgitter übergeben. Sind die Streuraten innerhalb des elektronischen Systems geringer als die Elektron-Phonon-Streuung, so kann eine Thermalisierung des lokal angeregten elektronischen Systems auch maßgeblich durch letzteren Prozess stattfinden. Die zugehörigen Zeitskalen werden zurzeit für das vorliegende System der $\operatorname{Si}(111)(\sqrt{3} x \sqrt{ } 3)-\mathrm{Ag}$ Rekonstruktion intensiv untersucht [105]. 


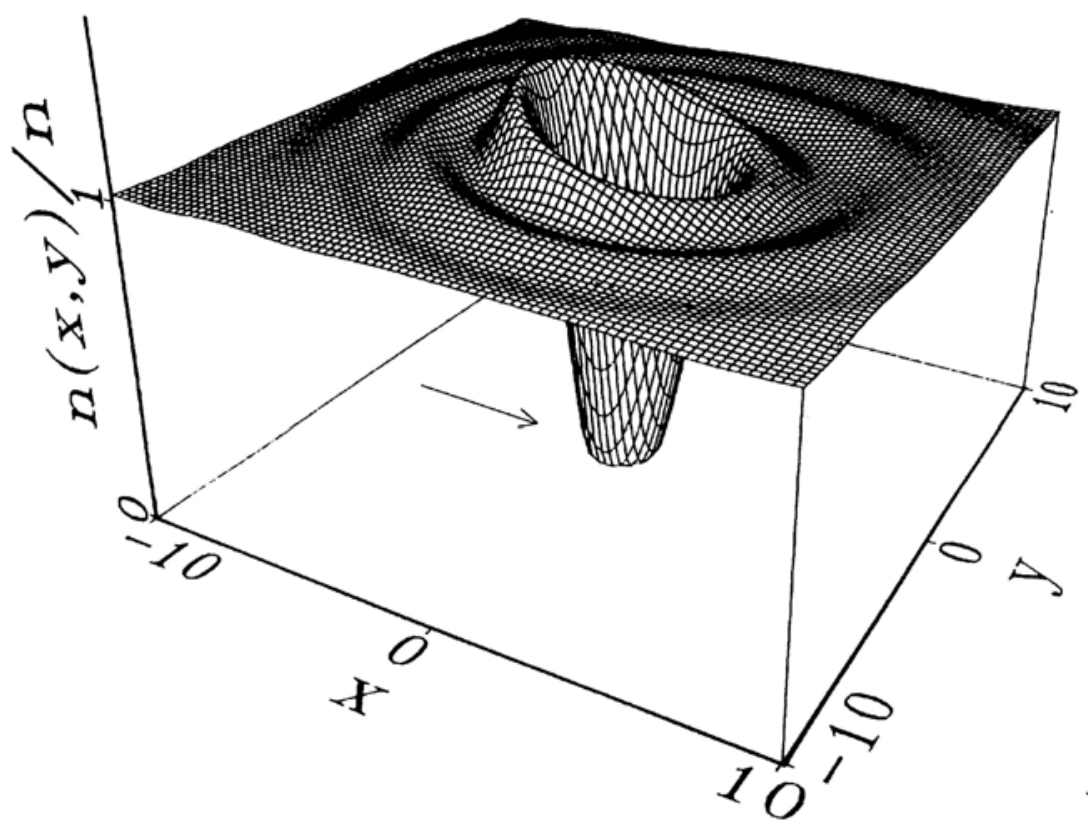

Abbildung 7: Streutheoretische, quantenmechanische Berechnung des Landauer-WiderstandsDipols nach Ref. [46]. Dargestellt ist die normalisierte Ladungsdichte in der Umgebung einer Scheibe mit $k_{f} a=1$. Der elektrische Strom fließt in positive $x$-Richtung (Driftgeschwindigkeit $v_{D} / v_{f}=0.2$. Die Längen sind in Einheiten des Scheibenradius a angegeben. 


\subsubsection{Charakteristische Längenskalen des Ladungstransports}

In den vorangegangenen Kapiteln wurde eine Vielzahl von unterschiedlichen Längenskalen angesprochen, so dass es notwendig erscheint, eine kurze Übersicht der charakteristischen Längenskalen anzugeben. Sie werden häufig verwendet werden, um den Ladungstransport zu klassifizieren:

Diffuser Transport $l_{M F P} \ll l_{\text {System }}$ : Elektronen erfahren beim Transport durch das System eine Vielzahl von diffusen Streuprozessen. Eine Modellierung geschieht meist in Form des DrudeSommerfeld-Modells.

Ballistischer Transport $l_{M F P} \gg l_{\text {System }}$ : Elektronen durchqueren das System ballistisch. Streuung findet nur an den Systemgrenzen statt. Eine Modellierung geschieht meist in Form des Modells nach Landauer-Büttiker.

Quantenmechanischer Transport $l_{\text {System }} \leq \lambda_{f} \ll l_{\phi}$ : Die Systemgröße ist vergleichbar mit der der Fermiwellenlänge $\lambda_{\mathrm{f}}$ und wesentlich kleiner als die Phasenkohärenzlänge $1_{\Phi}$. Interferenzeffekte dominieren und eine klassische Beschreibung versagt komplett, so dass der Transport vollständig quantenmechanisch beschrieben werden muss.

In realen Systemen ist eine Klassifizierung nach rein ballistischem oder rein diffusem Transport meist nicht möglich. Häufig verwendetem man daher die folgenden Begriffe um eine Mischform der unterschiedlichen konzeptionellen Beschreibungsarten, beim elektrischen Transport durch eine gegebene Probe, anzudeuten:

Makroskopischer Ladungstransport $l_{S y s t e m} \gg l_{M F P}, \lambda_{f}, l_{\phi}$ : Die Systemgröße ist wesentlich länger als alle relevanten Längenskalen und der Ladungstransport kann z.B. durch eine Volumenleitfähigkeit beschrieben werden.

Mesoskopischer Ladungstransport $\lambda_{f} \ll l_{\text {System }} \leq l_{M F P}, l_{\phi}$ : Quantenmechanische Interferenzeffekte können wichtig sein. $\lambda_{\mathrm{f}}$ ist jedoch wesentlich kleiner als die Systemgröße, so dass eine semiklassische Beschreibung gerechtfertigt sein kann. 
Die Raster-Tunnel-Potentiometrie ist derzeitig die einzige Methode, die den Ladungstransport auf allen charakteristischen Längenskalen analysieren kann und damit in der Lage ist, den experimentellen Übergang von der mikroskopischen zur makroskopischen Längenskala abzubilden. Der Messprozess der Raster-Tunnel-Potentiometrie bildet den Schwerpunkt des nächsten Abschnitts.

\subsubsection{Bestimmung lokaler elektrochemischer Potentiale mit Hilfe der Raster-Tunnel-Potentiometrie}

Um bei einer stromdurchflossenen Probe die experimentelle Messgröße interpretieren zu können, wird das chemische Potential der Probe in quasi-Potentiale (siehe Abbildung 5 und Abbildung 8) für die $\mathrm{k}^{+}$und $\mathrm{k}^{-}$-Zustände überführt [39]. ${ }^{10}$ Damit lassen sich ortsabhängige elektrochemische Potentiale $\mu_{e c}^{k^{+}, k^{-}}$angeben, die sich jeweils aus der Summe des lokalen elektrostatischen Potentials $\Phi(\mathrm{x}, \mathrm{y})$ sowie des lokalen chemischen Potentials $\mu_{c}^{k^{+}, k^{-}}$ergeben zu

$$
\mu_{e c}^{k^{+}}(x, y)=\mu_{c}^{k^{+}}(x, y)-e \phi(x, y) \text { und } \mu_{e c}^{k^{-}}(x, y)=\mu_{c}^{k^{-}}(x, y)-e \phi(x, y) .^{11}
$$

Um bei inhomogenen Systemen ein ortsabhängiges quasi-chemisches und auch elektrochemisches Potential angeben zu können, wird postuliert, dass sich die Potentiale in kleinen räumlichen Gebieten in einem dynamischen Gleichgewicht befinden und sich dementsprechend mit quasi-Gleichgewichtsgrößen wie $\mu_{c}^{k^{+}, k^{-}}$beschreiben lassen.

Abbildung 8 (b) skizziert den Messprozess der Raster-Tunnel-Potentiometrie. Das elektronische System der STM-Spitze befindet sich im Gleichgewicht und die $\mathrm{k}^{+}-$und $\mathrm{k}^{-}-$ Zustände sind gleich besetzt, so dass das elektrochemische Potential der Spitze eine wohldefinierte Gleichgewichtsgröße ist. Die Spitze koppelt über den Tunnelkontakt schwach an das elektronische System der Probe am jeweiligen Ort der Spitze. Durch den externen Potentiometrie-Regelkreis wird das elektrochemische Potential der Spitze so eingestellt, dass im zeitlichen Mittel kein Netto-Strom zwischen Spitze und Probe fließt. In dieser Situation addieren sich die in die Spitze hinein und heraus tunnelnden Strom-Komponenten zu Null.

\footnotetext{
${ }^{10}$ Diskutiert wird die Situation für minimale Temperaturen. Andernfalls sind die Besetzungsfunktionen keine Kastenfunktionen und entsprechend der Fermi-Funktion besetzt.

${ }^{11}$ Das negative Vorzeichen resultiert aus der Elektronenladung q=-e.
} 
Der verschwindende Ladungsstrom darf jedoch nicht mit einem verschwindenden Energiestrom gleichgesetzt werden. Die hinein-tunnelnden Elektronen können eine größere Energie besitzen als die heraus-tunnelnden, was zu einem Netto-Energietransport ohne Ladungstransport führen kann.

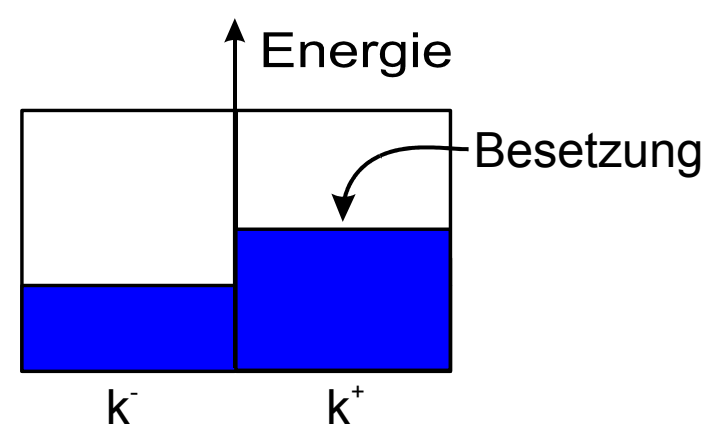

a)

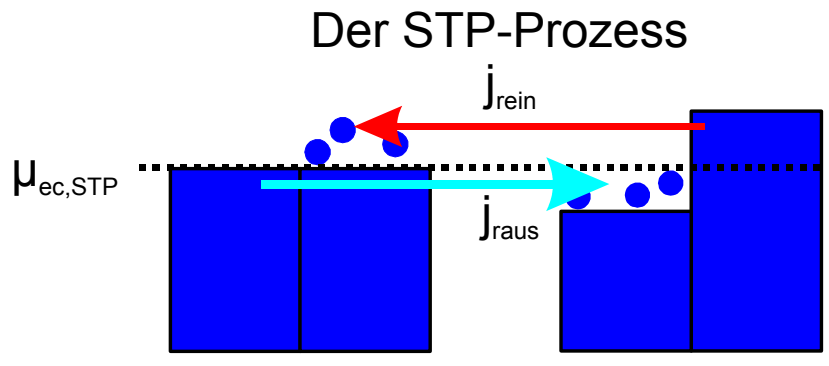

Spitze
Probe

b)

Abbildung 8: (a) Aufteilung der Besetzung in quasi-chemische Potentiale für die $k^{+}$und $k^{-}$ Zustände bei tiefen Temperaturen nach Ref. [39]. Die skizzierte Besetzung entspricht einem Netto-Elektronentransport von links nach rechts. In (b) ist eine Schemaskizze des STPMessprozesses gezeigt. Das elektrochemische Potential der Spitze wird derart angepasst, dass kein Netto-Strom zwischen Spitze und Probe fließt. In diesem Sinn wird durch $\mu_{e c, S T P}$ das lokale elektrochemische Potential der Probe abgebildet.

Das elektrochemische Potential der Probe, welches sich im Nicht-Gleichgewicht befindet, wird dadurch mit dem elektrochemischen Potential der Spitze abgeglichen. Hierbei erfolgt ein nicht näher bekannter Mittelungsprozess über die quasi-elektrochemischen Potentiale der $\mathrm{k}^{+}-$ und $\mathrm{k}^{-}$-Zustände der Probe. Unter Vernachlässigung einer energieabhängigen Transmission durch die Tunnelbarriere wird der einfache Mittelwert $\mu_{e c, S T P}=1 / 2\left(\mu_{e c}^{k^{+}}+\mu_{e c}^{k-}\right)$ als Arbeitshypothese für den Mittelungsprozess benutzt, wie er auch in Ref. [39] (Seite 71) verwendet wurde. Das Kompensationspotential wird im Verlauf dieser Arbeit mit $\mu_{\mathrm{ec}, \mathrm{STP}}$ bezeichnet, um anzudeuten, dass es sich hierbei um keine Gleichgewichtsgröße handelt. 


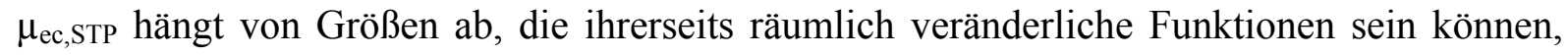
wobei die Poissongleichung das elektrostatische Potential mit der Ladungsdichte verknüpft. Die eindeutige Interpretation von $\mu_{\text {ec,STP }}$ ist daher schwierig. Sie kann jedoch, wie die folgenden Beispiele zeigen, in Grenzfällen angegeben werden:

\section{a) Diffuser Transport mit ausschließlich delokalisierter Streuung:}

Abbildung 9 zeigt schematisch das chemische Potential und das elektrostatische Potential innerhalb eines stromdurchflossenen Leiters mit ausschließlich diffuser Streuung gemäß dem Drude-Sommerfeld-Modell. Die quasi-chemischen Potentiale sind ortsunabhängig, so dass $\mu_{\mathrm{ec}, \mathrm{STP}}$ dem Verlauf des elektrostatischen Potentials folgt:

$$
\mu_{e c, S T P}(x, y)=1 / 2\left(\mu_{e c}^{k^{-}}+\mu_{e c}^{k^{-}}\right) \sim-e \phi(x, y)
$$

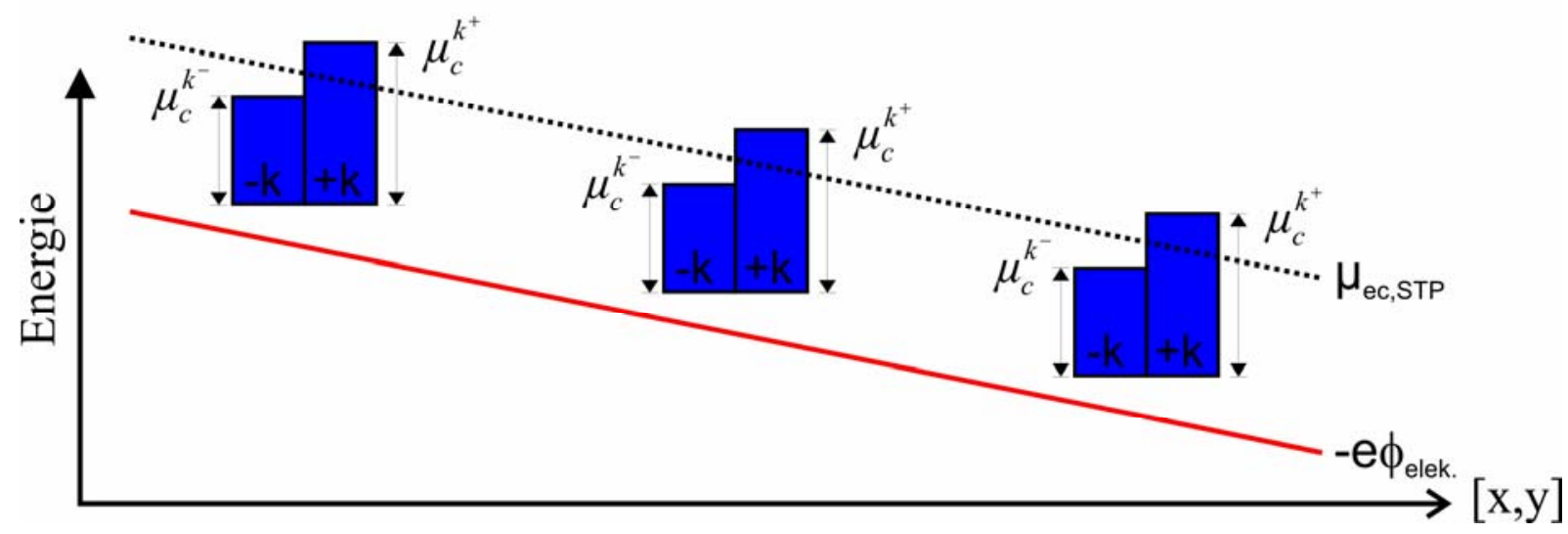

Abbildung 9: Schemaskizze zum Verlauf des elektrostatischen und des chemischen Potentials innerhalb eines Leiters mit ausschließlich delokalisierter Streuung (Diffuser Transport gezeichnet im Rahmen des Drude-Sommerfeld-Modells). Die quasi-chemischen Potentiale sind ortsunabhängig und das elektrochemische Potential folgt dem elektrostatischen Potential $\mu_{e c, S T P}(x, y) \sim-e \phi(x, y)$. 


\section{b) Ballistischer Transport mit lokalisierter Streuung:}

Abbildung 10 zeigt schematisch $\mu_{c}^{k^{+}, k^{-}}(\mathrm{x}, \mathrm{y}),-\mathrm{e} \Phi(\mathrm{x}, \mathrm{y})$ und $\mu_{\mathrm{ec}, \mathrm{STP}}(\mathrm{x}, \mathrm{y})$ in einem ballistischen System mit einer quantenmechanischen Tunnelbarriere nach Ref. [39]. Die Kontaktbereiche werden nicht weiter betrachtet und es wird davon ausgegangen, dass in $(a, h)$ die entsprechenden Besetzungen vorliegen. Fern der Barriere, d. h. außerhalb der elektrostatischen Abschirmlänge, ist $\Phi(\mathrm{x}, \mathrm{y})$ konstant. Die damit selbstkonsistent verknüpften quasi-chemischen Potentiale sind außerhalb der elektrostatischen Abschirmlänge entsprechend konstant (siehe $(\mathrm{a}, \mathrm{b}, \mathrm{g}, \mathrm{h})$ ). Die Nicht-Gleichgewichtsverteilung an diesen Orten korrespondiert zu dem fließenden elektrischen Strom. Links der Barriere transmittieren Elektronen der $\mathrm{k}^{+}$-Zustände mit der Wahrscheinlichkeit $\mathrm{T}$ durch die Barriere zu (e) oder werden mit der Wahrscheinlichkeit (1-T) reflektiert (siehe (d)). Reflektierte Elektronen laufen in k-Richtung und verändern dadurch die Besetzung der entsprechenden quasi-chemischen Potentiale (a-d). Schematisch ist die Besetzung nach Energierelaxation gezeigt. Die Quelle für Elektronen der k-Zustände links der Barriere sind ausschließlich von der Barriere reflektierte Elektronen. Die Quellen für die $\mathrm{k}^{+}$-Zustände rechts der Barriere sind mit der Wahrscheinlichkeit $\mathrm{T}$ transmittierte Elektronen aus (d) und reflektierte Elektronen der $\mathrm{k}^{-}$-Zustände rechts der Barriere (e). Im skizzierten Fall beträgt die Reflektion der k-Zustände von (e) nach (d) 100\%, da bei (e) keine freien Zustände verfügbar sind.

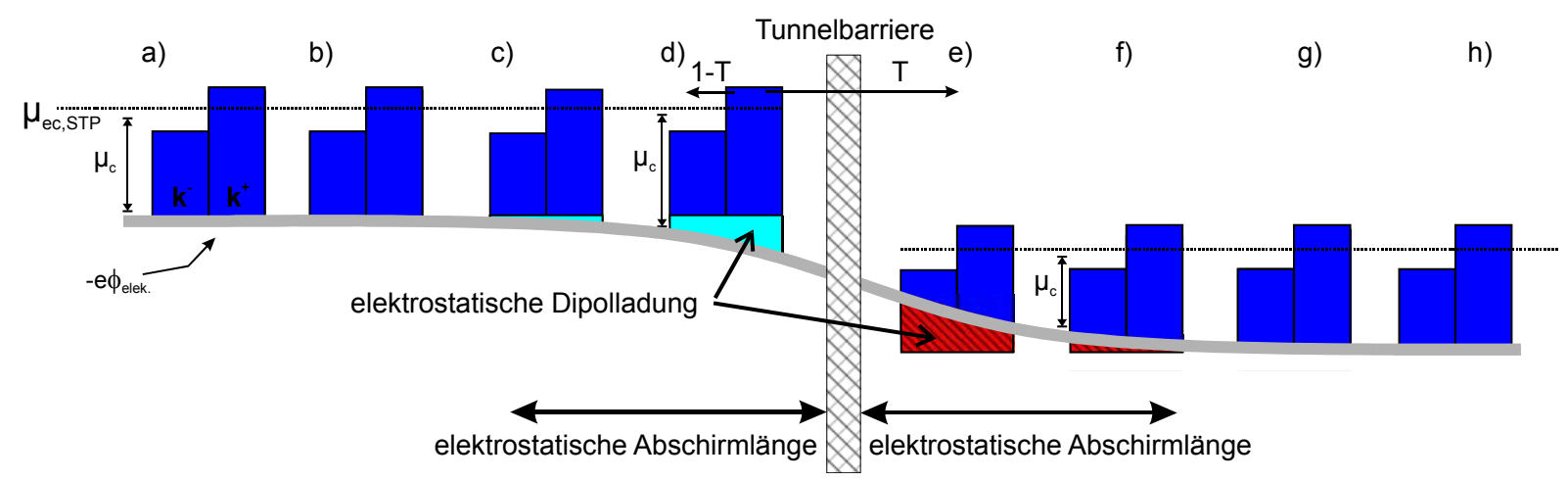

Abbildung 10: Schemaskizze zum Verlauf des elektrostatischen und des chemischen Potentials innerhalb eines ballistischen Leiters mit einer Barriere: Fern der Barriere ist das chemische, elektrostatische und deswegen auch das elektrochemische Potential konstant. Am Ort der Barriere (d, e) variiert das elektrostatische Potential innerhalb der Abschirmlänge und es kommt zur Ausbildung eines elektrostatischen Dipols. Selbstkonsistent mit der Ladungsdichte des Dipols variiert das chemische Potential, so dass $\mu_{\text {ec }}$ bis zur Barriere konstant ist. 
Am Ort der Barriere variiert sowohl $\mu_{c}^{k^{+}, k^{-}}(x, y)$ als auch $\Phi(\mathrm{x}, \mathrm{y})$, wobei die Ladungsdichte $\rho$ über die Poisson-Gleichung $\Delta \phi(\vec{r})=-\frac{\rho(r)}{\varepsilon_{0}}$ mit dem elektrostatischen Potential verknüpft ist. Ohne Transport wäre $\mu_{\mathrm{ec}, \mathrm{STP}}(\mathrm{x}, \mathrm{y})$ konstant, so dass eine Veränderung in $\Phi(\mathrm{x}, \mathrm{y})$ mit einer Veränderung in $\mu_{c}^{k^{+}, k^{-}}(x, y)$ korrespondieren würde. Datta postuliert, dass das selbstkonsistente Variieren von $\mu_{c}^{k^{+}, k^{-}}(x, y)$ und $\Phi(\mathrm{x}, \mathrm{y})$ auch auf das durch den Transport gestörte System zutrifft. Das elektrochemische Potential auf beiden Seiten ist demnach konstant und variiert „almost discontinuously“ (Ref. [39], Seite 73) am Ort der Barriere um $\Delta \Phi$, wobei $\Delta \Phi$ außerhalb der elektrostatischen Abschirmlänge evaluiert werden muss. Eine experimentelle Verifikation steht jedoch bis dato noch aus und wird in Kapitel 4 dieser Arbeit erstmalig diskutiert. 


\subsubsection{Experimentelles Auflösungsvermögen}

Bevor das experimentelle Auflösungsvermögen der vorhandenen Anlage anhand von Testmessungen auf der $\operatorname{Si}(111)(\sqrt{3} \mathrm{x} \sqrt{ } 3)$-Ag Rekonstruktion spezifiziert wird, erscheint es sinnvoll zunächst die intrinsischen Rauschquellen der STP-Apparatur zu betrachten. Dazu werden die verschiedenen Komponenten einzeln charakterisiert und sukzessive zur vollständigen Beschaltung zusammengeführt. Abbildung 11 zeigt die relevanten Komponenten des verwendeten Testaufbaus.

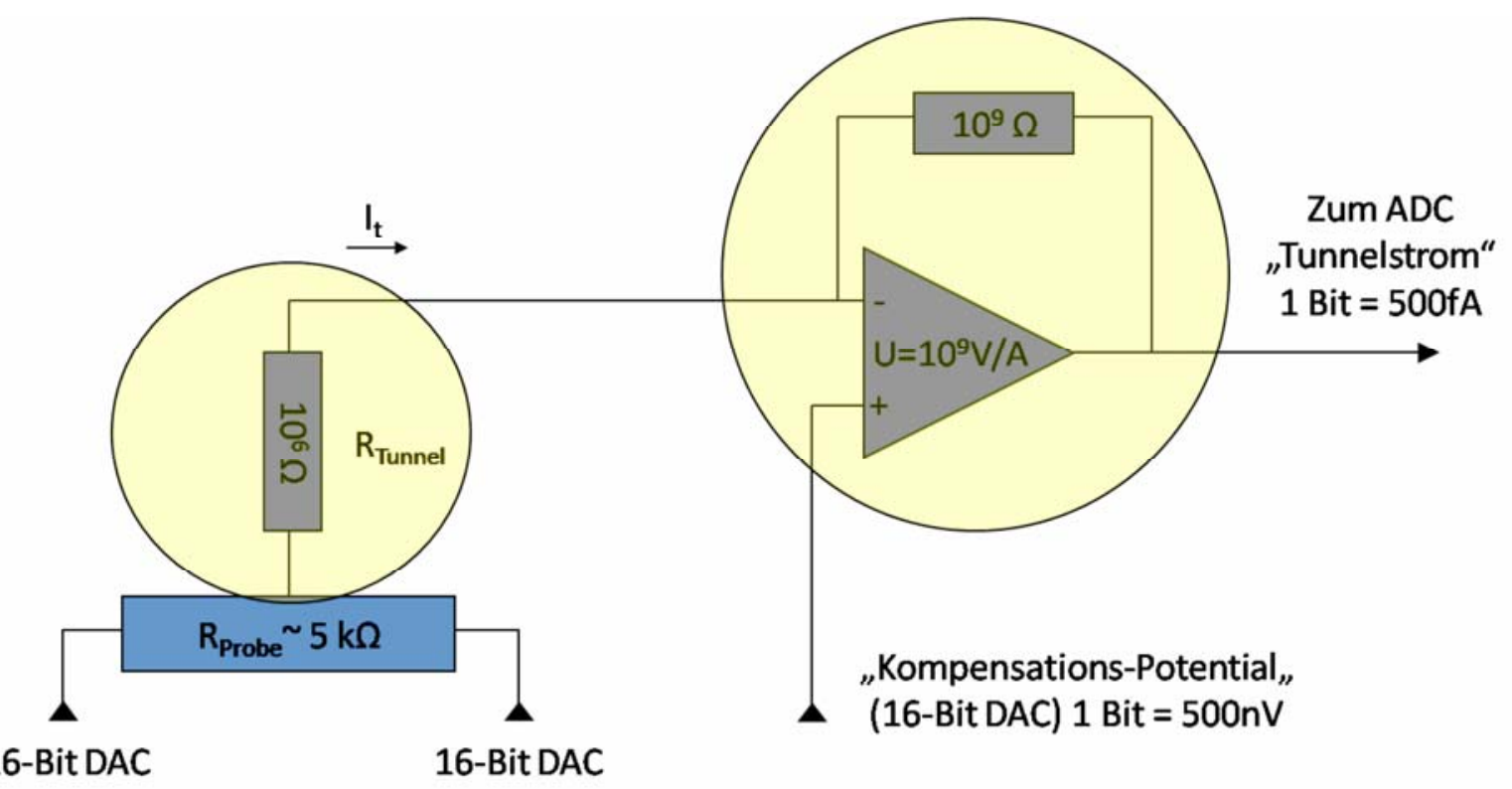

Abbildung 11: Schematische Schaltskizze der relevanten STP-Komponenten während einer Potentiometrie-Messung.

Die Probe wird als Widerstandsteiler modelliert und koppelt lokal über einen Tunnelwiderstand von $\mathrm{R}_{\mathrm{t}}=10^{6} \Omega$ an den Eingang des Stromverstärkers. Der linke und rechte Kontakt der Probe wird mit zwei 16-Bit DACs über die Leistungsstufe angesteuert. Das Kompensationspotential wird über die virtuelle Masse des Stromverstärkers ebenfalls mit einem 16-Bit DAC mit anschließendem 1:10000 Teiler kontrolliert. Der Tunnelstromverstärker ist als Strom-Spannungs-Wandler mit einem Rückkoppelwiderstand von $10^{9} \Omega$ beschaltet. Die Ausgangsspannung des Operationsverstärkers stellt sich bei dieser Art der Beschaltung so ein, dass beide Eingänge sowie der Ausgang auf demselben Potential liegen. Beim Null-Abgleich des Tunnelstroms ist daher die anliegende Kompensationsspannung am Ausgang mit zu berücksichtigen. Bei der Kompensationsmethode erfolgt die Evaluierung des Probenpotentials stromlos, d. h. sowohl der Rückkoppelwiderstand als auch der Tunnelwiderstand wird nicht 
von einem Netto-Strom durchflossen. Dies ist ein großer Vorteil gegenüber Methoden bei denen ein Netto-Strom fließt. Abbildung 12 illustriert diesen Sachverhalt an gemessenen Rauschspektren des Tunnelstrom für verschiedene Parameter [47]. Demnach ergibt sich das Stromrauschen $\Delta \mathrm{I}_{\mathrm{t}}$ am Tunnelwiderstand $\mathrm{zu} \Delta I_{t}=\operatorname{coth}\left(e U_{\text {Bias }} / 2 k_{b} T\right)[47,48]$. Bei großen Tunnelströmen dominiert Schrot-Rauschen, welches eine direkte Konsequenz der LadungsQuantisierung des Elektrons ist [47, 49-52]. Bei kleinen Tunnelströmen $\left(e\left|R_{t} I_{t}\right| \ll k_{b} T\right)$ hingegen, sättigt die Rauschamplitude beim thermischen Rauschen (Johnson-NyquistRauschen) $\Delta I_{\text {Johnson-Nyquist }}=\sqrt{4 k_{b} T f_{b w} / R}$, wobei $\mathrm{f}_{\mathrm{bw}}$ die Bandbreite bezeichnet [53, 54]. Aufgrund dieser Tatsache ist die im Rahmen dieser Arbeit verwendete Kompensationsmethode anderen strombehafteten Potential-Messverfahren überlegen und die Potentialmessungen können dadurch bei physikalisch minimalen Rauschbedingungen ausgeführt werden.

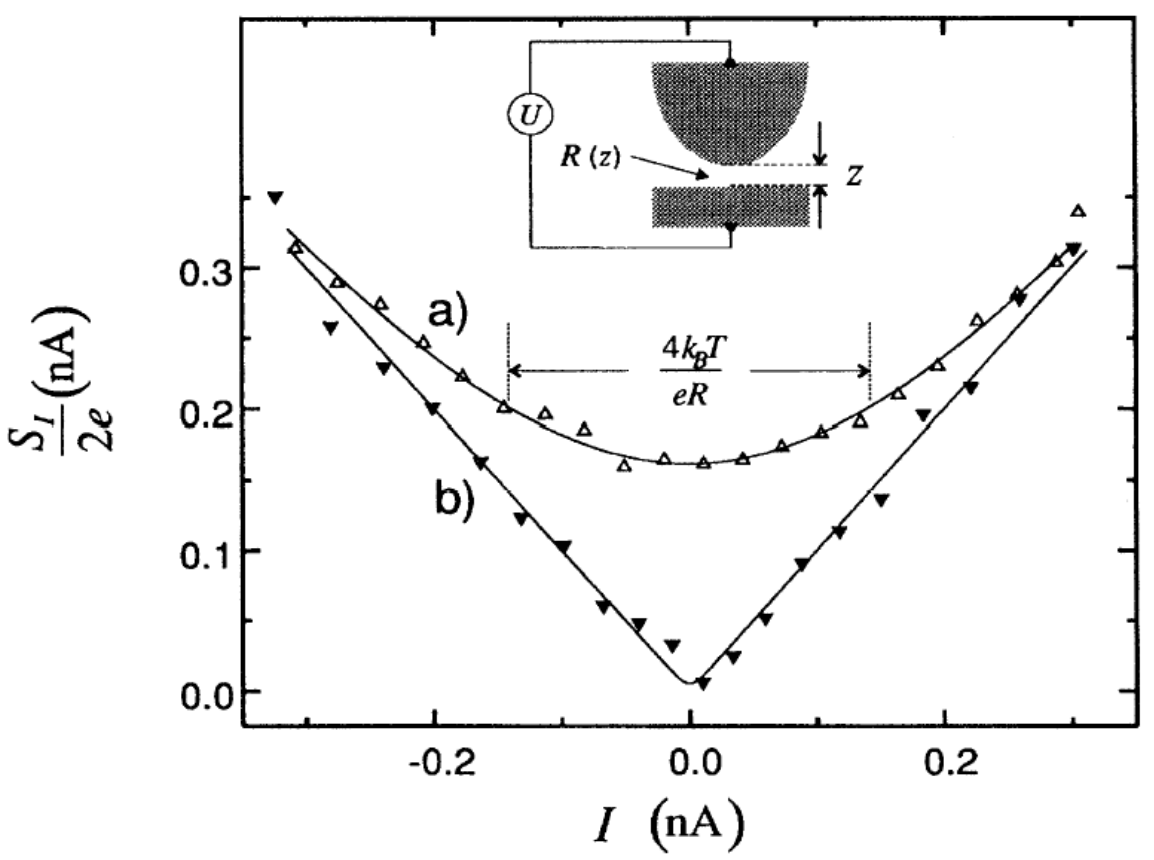

Abbildung 12: Gemessenes Stromrauschen am Tunnelkontakt bei $200 \mathrm{kHz}$ Bandbreite als Funktion des Tunnelstroms. Ein Übergang vom thermischen Rauschen zum Schrot-Rauschen ist ersichtlich. (a) Zeigt eine Messung bei $300 \mathrm{~K}$ und $R_{t}=0,32 \mathrm{G} \Omega$ und (b) bei $77 \mathrm{~K}$ und $R_{t}=2,7$ GQ. Abbildung aus Ref. [47]. 
Nachdem das Rauschverhalten des Tunnelstroms thematisiert worden ist, soll nun das Rauschen des Tunnelstromverstärkers an sich diskutiert werden. Das thermische Rauschen des Rückkoppelwiderstands $\left(10^{9} \Omega\right)$ beträgt bei der verwendeten Bandbreite von $50 \mathrm{kHz}$ theoretisch $900 \mathrm{fA}$, was gleichsam die physikalische Auflösungsgrenze des Tunnelstromverstärkers ist. Experimentell werden $2200 \mathrm{fA}$ ohne Eingangswiderstand gemessen, so dass der Tunnelstromverstärker an sich bis auf den Faktor $\sim 2$ am physikalischen Rauschlimit arbeitet.

Abbildung 13 illustriert das Rauschen des Stromverstärkers inklusive simuliertem Tunnelwiderstand. Dazu wurde sein Eingang mit einem $1 \mathrm{M} \Omega$ Metallfilm-Widerstand und die virtuelle Masse (Kompensationseingang) kurzgeschlossen. ${ }^{12}$ Messwerte für zwei experimentelle Bandbreiten sind im Vergleich mit dem theoretischen Stromrauschen gezeigt. Auch in diesem Fall liegt das experimentelle Rauschen lediglich um den Faktor 2 höher als das physikalische Rauschlimit.

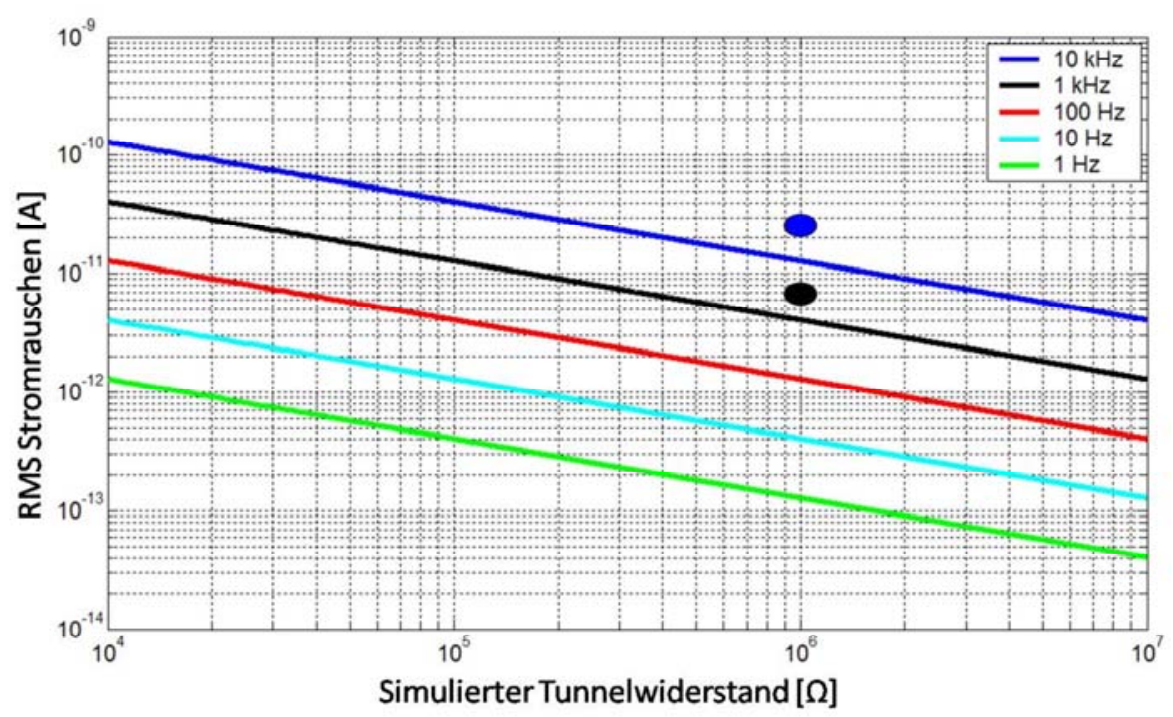

Abbildung 13: Zur Charakterisierung des Stromverstärkers wurde sein Eingang mit 1MQ kurzgeschlossen. Dies simuliert den Tunnelwiderstand. Bei einer Bandbreite von $1 \mathrm{kHz}$ beträgt das experimentelle Stromrauschen $6 \mathrm{pA}$ (schwarzer Punkt). Bei $10 \mathrm{kHz}$ Bandbreite ist das experimentelle Rauschen 25 pA (blauer Punkt). Zum Vergleich ist das theoretische Stromrauschen gemäß $\Delta I_{\text {Johnson-Nyquist }}=\sqrt{4 k_{b} T f_{b w} / 1 M \Omega}$ gezeigt.

\footnotetext{
${ }^{12}$ Generell dürfen nur Metallfilm-Widerstände verwendet werden, um zusätzliche 1/f-
} Komponenten zu vermeiden. 
Abbildung 14 zeigt zudem die spektrale Stromdichte bei $\sim 2 \mathrm{kHz}$ Bandbreite. Das gemessene Rauschspektrum ist nahezu frequenzunabhängig und kann deshalb als thermisches Rauschen klassifiziert werden. Der Tunnelstromverstärker an sich und mit simuliertem Tunnelwiderstand ist somit gut charakterisiert.

Im nächsten Schritt des Aufbaus zur vollständigen STP-Anlage wird der Kompensationseingang des Stromverstärkers über einen 1:1000 Teiler beschaltet. Hierdurch steigt die spektrale Stromdichte um fast zwei Dekaden an. Das Spektrum ähnelt jedoch nach wie vor einem thermischen Rauschen. Das Einschalten des Potentiometrie-Regelkreises regelt die tiefen Frequenzen natürlicherweise gut aus, so dass die spektrale Stromdichte bei den tiefen Frequenzen mit Regelkreis vermindert erscheint. Die gezeigten Daten lassen bis zu diesem Punkt den Schluss zu, dass die STP-Messung ausschließlich durch thermisches Rauschen beeinflusst wird. Eine längere Mittelungszeit sollte dementsprechend die Auflösung der STPMessung gemäß $\Delta \mu_{e c, S T P} \sim 1 / \sqrt{N}$ beliebig verbessern. Diesen Sachverhalt beleuchtet Abbildung 15. Die Beschaltung entspricht nun der gesamten Komplexität einer vollständigen STP-Messung. Für die Analyse wurde der Tunnelstrom ohne Potentiometrie-KompensationsRegelkreis aufgezeichnet.

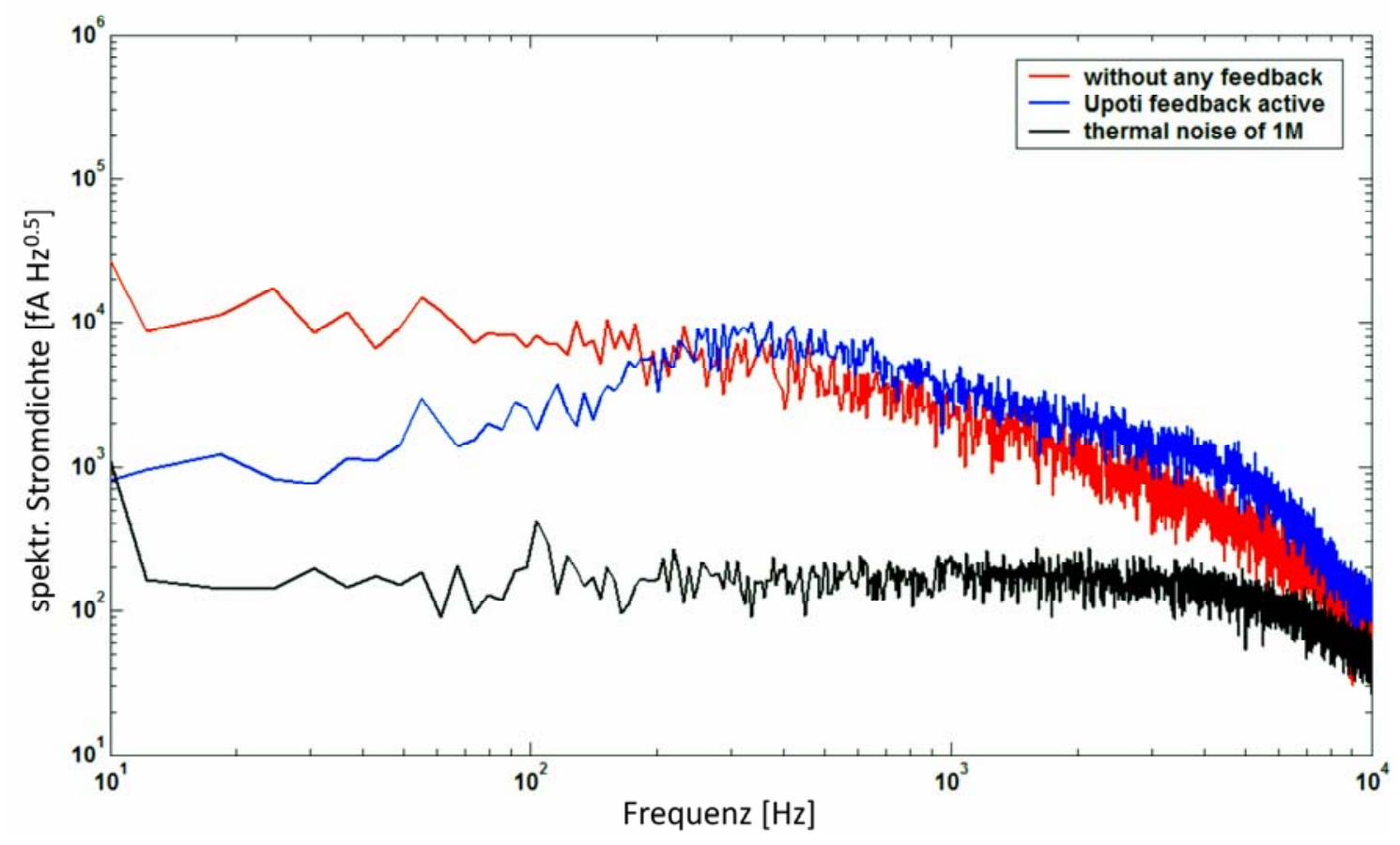

Abbildung 14: Spektrale Stromdichte des Tunnelstroms bei $2 \mathrm{kHz}$ Bandbreite und vollständiger STP-Beschaltung gemäß Abbildung 11. 
Dargestellt ist die spektrale Stromdichte des Tunnelstroms im Frequenzspektrum von $\left(10^{-4} \ldots 10^{2}\right) \mathrm{Hz}$. Im Bereich von $20 \mathrm{~Hz}$ bis $100 \mathrm{~Hz}$ wird thermisches Rauschen mit konstanter Rauschamplitude beobachtet. Unterhalb von $20 \mathrm{~Hz}$ steigt die spektrale Stromdichte allerdings linear mit dem Kehrwert der Frequenz an (1/f-Rauschen). Eine Rauschverminderung gemäß $\Delta \mu_{e c, S T P} \sim 1 / \sqrt{N}$ ist demnach nur für Mittelungszeiten bis maximal $50 \mathrm{~ms}$ zu erwarten. Bei dieser Mittelungszeit beträgt das Stromrauschen ca. $200 \mathrm{fA}$, so dass die theoretische Auflösungsgrenze der Potentiometrie-Messung bei $\mathrm{R}_{\mathrm{t}}=1 \mathrm{M} \Omega \mathrm{zu} 200 \mathrm{nV}$ abgeschätzt werden kann.

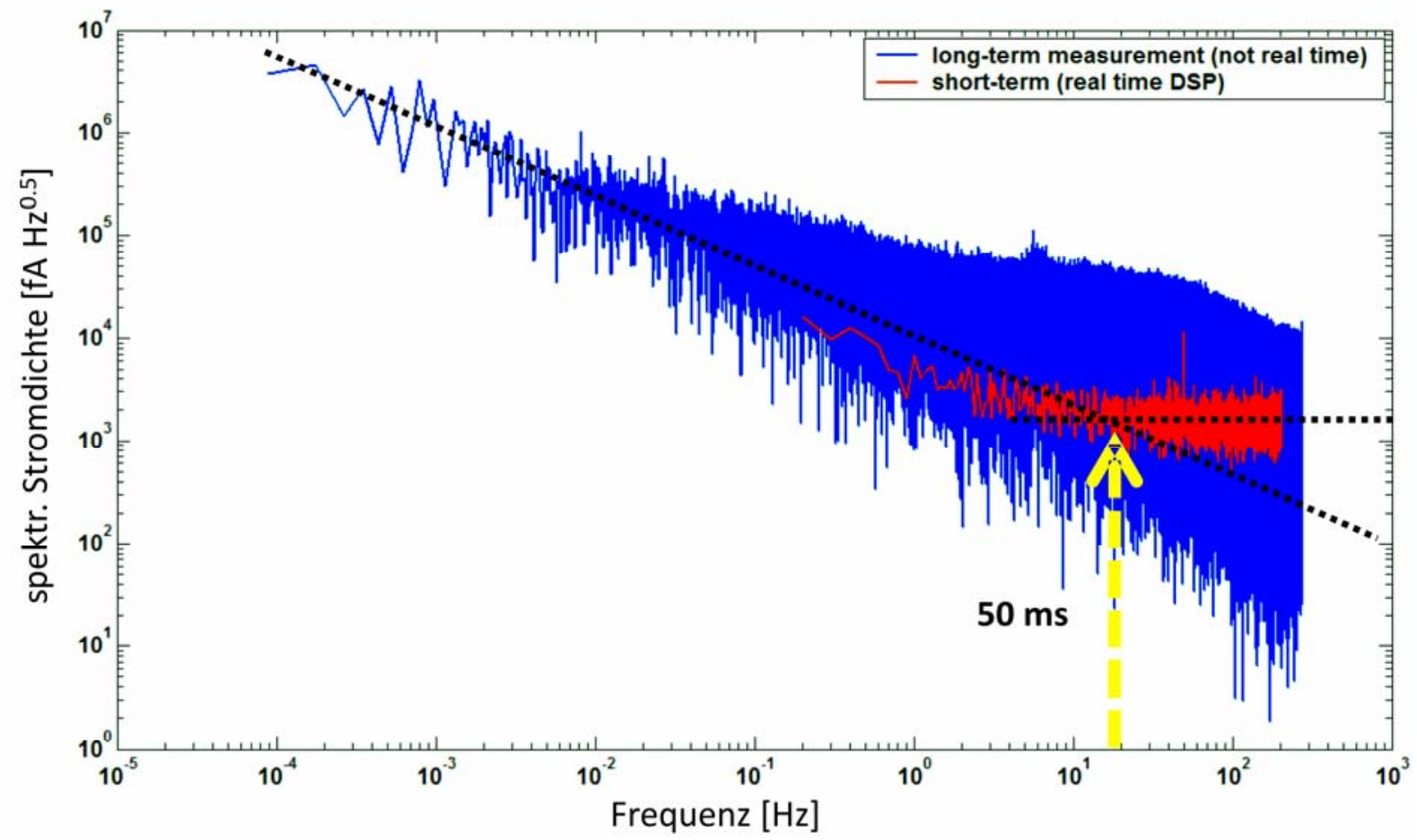

Abbildung 15: Spektrale Stromdichte des Tunnelstroms bei vollständiger Beschaltung für eine STP-Messung entsprechend Abbildung 11 bei $R_{t}=1$ MS. Der Tunnelstrom wurde ohne Potentiometrie-Kompensations-Regelkreis aufgezeichnet. Bei hohen Samplingraten geschah dies in Echtzeit auf der DSP-Karte, bei geringeren Samplingraten dafür aber über Stunden mit einem herkömmlichen ADC über den PC. 
Eine Charakterisierung der Langzeitstabilität des Potentiometrie-Aufbaus zeigt Abbildung 16. Die Potentiometrie-Daten wurden über 3 Tage kontinuierlich mit einer Samplingrate von $5 \mathrm{~ms}$ aufgezeichnet. In diesem Zeitraum schwankt das gemessene Potential um fast $100 \mu \mathrm{V}$. Wie der Inset zeigt, sind die Potentialschwankungen nicht normalverteilt, sondern bestehen im Wesentlichen aus drei diskreten Niveaus bei $230 \mu \mathrm{V}, 270 \mu \mathrm{V}$ und $290 \mu \mathrm{V}$. Die Niveaus treten auch direkt am Ausgang der analogen Leistungsstufe mit kurzgeschlossenem Eingang auf. ${ }^{13}$

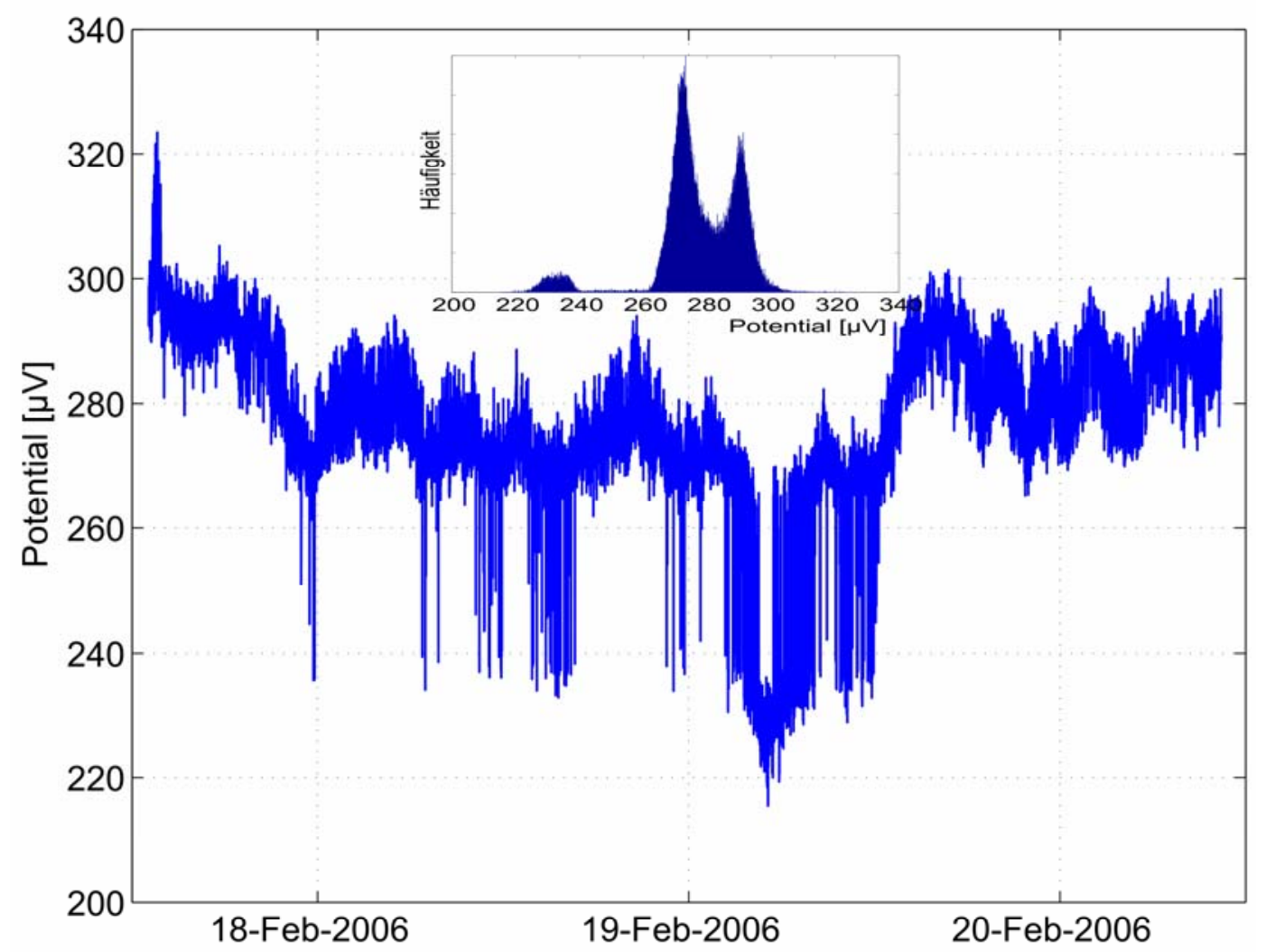

Abbildung 16: Potential-Testmessung entsprechend Abbildung 11 mit 5 ms Mittelungszeit je Messpunkt. Dargestellt ist ein Zeitraum von ca. 3 Tagen. Zur Darstellung wurde jeweils über 256 Messpunkte entsprechend 1,28 s gemittelt. Das Kompensationspotential schwankt innerhalb dieser Zeit um $100 \mu \mathrm{V}$. Das Histogramm illustriert die Häufigkeitsverteilung des Kompensationspotentials (Rohdaten) und zeigt im Wesentlichen drei diskrete Niveaus bei $230 \mu \mathrm{V}, 270 \mu \mathrm{V}$ und $290 \mu \mathrm{V}$ mit einem FWHM von jeweils ca. $5 \mu \mathrm{V}$.

${ }^{13}$ Als Leistungstreiber wird der extrem rauscharme, bidirektionale und monolithische Hybridverstärker PA26 der Firma APEX eingesetzt. Seine Temperaturkonstanz beträgt typischerweise $10 \mu \mathrm{V} /{ }^{\circ} \mathrm{C}$. 
Solch ein Rauschverhalten ist in der Literatur als „Burst-Noise“ bekannt und noch immer nicht vollständig verstanden [55]. „Burst-Noise“ tritt bei allen Halbleiterbauelementen in unterschiedlichem Ausmaß auf und wird mit der Umladung von Störstellen in Verbindung gebracht. Charakteristisch für „Burst-Noise“ sind zeitlich unkorrelierte Schwankungen zwischen zwei oder mehreren diskreten Niveaus bei niedrigen Frequenzen (Größenordnung $100 \mathrm{~Hz}$ ). Eine Minimierung dieser Rauschquelle wurde dadurch erreicht, dass eine Vielzahl von Hybridverstärkern aus unterschiedlichen Chargen gekauft und analysiert worden sind, um die Besten davon für den Versuchsaufbau zu verwenden.

Darüberhinaus wurde bei langen Messkampagnen eine Korrelation des Potentiometrie-Signals mit der Tag/Nacht-Absenkung der Heizung sowie des Regelverhaltens der Klimaanlage beobachtet. Dies liegt zum Einen an der Temperaturkonstanz der verwendeten Bauteile (sämtliche Widerstände, ADC, DAC, Leistungsstufe), wobei der Tunnelstromverstärker eine besonders hohe Temperaturabhängigkeit besitzt. Zum Anderen besitzen die elektrisch kontaktierten Komponenten von der Elektronik bis zum UHV-Kopf stark unterschiedliche Wärmekapazitäten und thermische Ankopplungen an die Raumluft, so dass Thermospannungen entlang der Signalwege ebenfalls in Betracht kommen. Elektrochemische Effekte wie „galvanische Zellen“ an Kontaktstellen wurden als weitere mögliche Störquellen untersucht. Letztendlich konnte eine Verbesserung erzielt werden, indem die Sonneneinstrahlung minimiert und auf eine aktive Regelung der Raumtemperatur verzichtet worden ist. Die Raumtemperatur veränderte sich dadurch während einer Messung ausreichend langsam und gleichmäßig. Auch die Kabelführung zwischen Messelektronik und STM hat einen erheblichen Einfluss auf das Rauschverhalten. So muss ein Abstand von ca. $50 \mathrm{~cm} \mathrm{zu}$ jeglichen Transformatoren und zum Raumheizkörper eingehalten werden.

Nachdem diese Rausch- bzw. Störquellen optimiert oder beseitigt worden sind, wurde das erreichte experimentelle Auflösungsvermögen anhand einer STP-Messung auf einer $\operatorname{Si}(111)(\sqrt{3} x \sqrt{3})-A g$ Probe ohne Querspannung bestimmt. Als Mittelungszeit der Potentiometrie-Messung wurden 20 ms gewählt. Abbildung 17 (a) zeigt die simultan zur Potentiometrie gemessene Topographie der Probe. Zwei monoatomare Stufen sind erkennbar. (b) zeigt das gemessene Potentiometrie-Bild. 


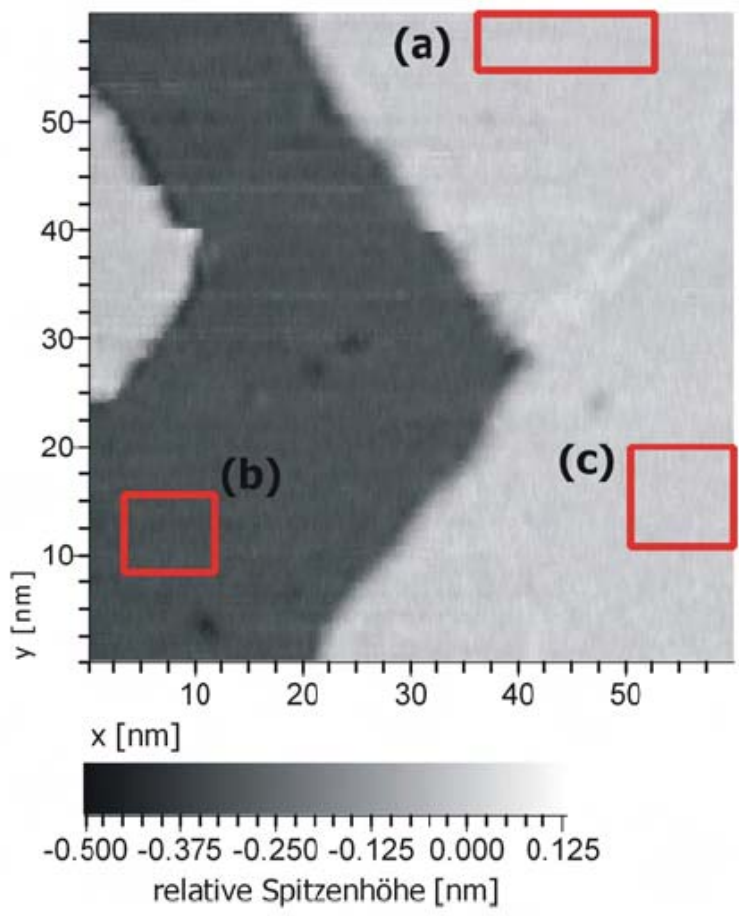

(a) Topografie

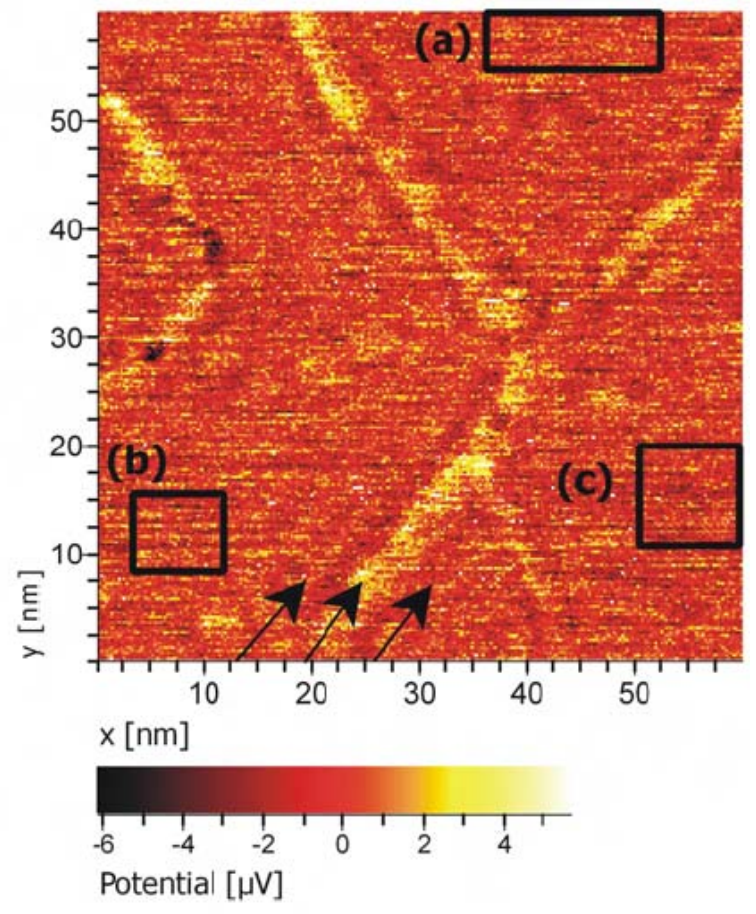

(b) Potentiometrie

Abbildung 17: STP-Testmessung auf der im weiteren Verlauf dieser Arbeit verwendeten Si(111)( $\sqrt{3} x \sqrt{ } 3)-A g$ Probe ohne Querspannung. (a) zeigt die Topographie und (b) das Potentiometrie-Bild mit $20 \mathrm{~ms}$ Mittelungszeit $\left(U_{\text {Bias }}=10 \mathrm{mV}, \mathrm{I}_{t}=2 \mathrm{nA}\right)$. Die Pfeile markieren Extrema einer leichten Thermospannungsoszillation (ca. $4 \mu \mathrm{V}$ Amplitude). Die einzelnen Bildbereiche markieren die Ausschnitte für Abbildung 18. Abbildung aus Ref. [36].

An den Stufenkanten ist eine Modulation mit einer Amplitude von ca. $4 \mu \mathrm{V}$ zu erkennen. Diese Oszillationen sind Thermospannungsoszillationen, die auftreten wenn Spitze und Probe unterschiedliche Temperaturen besitzen [56-60]. Thermospannungsoszillationen sind ein Störsignal bei der Analyse von Ladungstransportvorgängen mit Hilfe der Raster-TunnelPotentiometrie, da sie ebenfalls zu einem räumlich variierenden elektrochemischen-Potential führen. Die Amplitude der Thermospannungsoszillationen skaliert quadratisch zur Temperaturdifferenz zwischen Spitze und Probe, so dass für Ladungstransportuntersuchungen auf einen möglichst guten Wärmeabtrag der stromtragenden Struktur zu achten ist. ${ }^{14}$

\footnotetext{
${ }^{14}$ Ergebnisse, die im Zusammenhang mit Thermospannungsoszillationen entstanden sind, wurden als Physical Review B 76, 193407 (2007) veröffentlicht [34].
} 
Nach langem Suchen hat sich die $\operatorname{Si}(111)(\sqrt{3} x \sqrt{ } 3)-A g$ Rekonstruktion als ideales System erwiesen, da der Si-Wafer die entstehende Wärme sehr gut abführen kann.

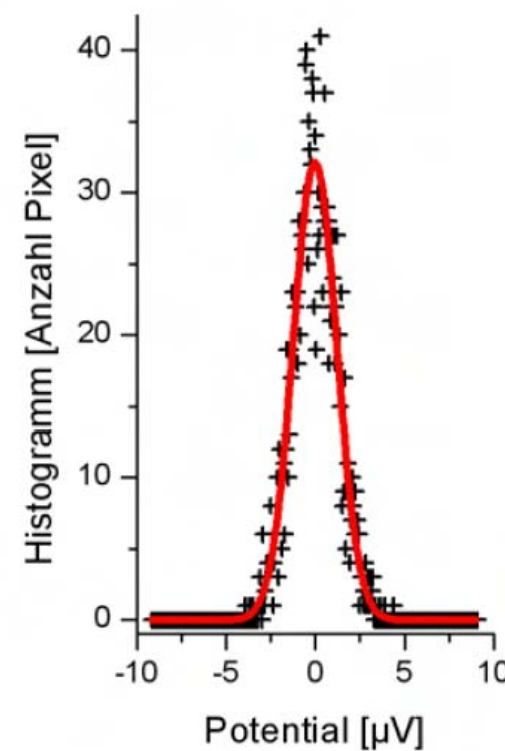

(a)

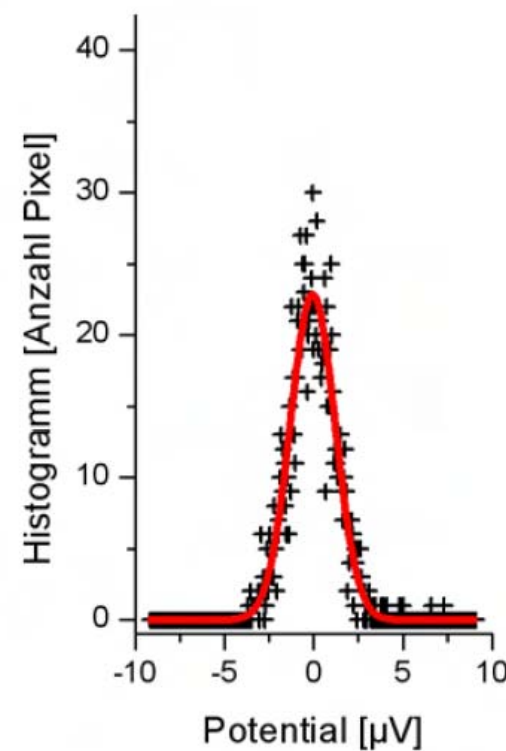

(b)

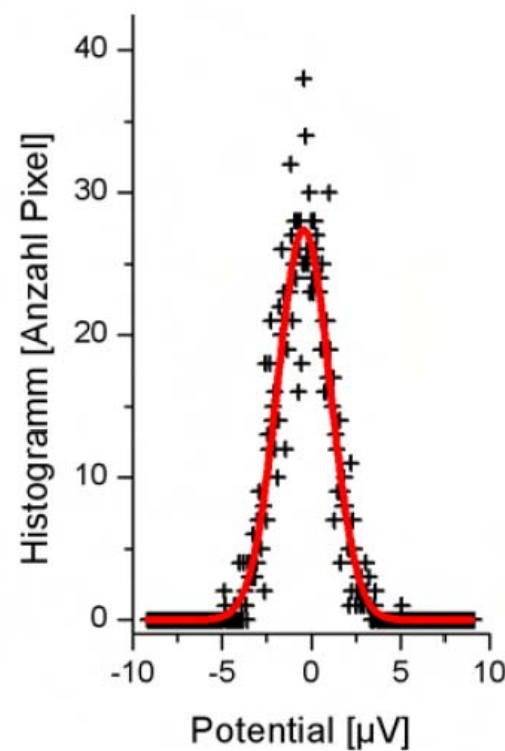

(c)

Abbildung 18: Auswertung zum experimentellen Auflösungsvermögen der STP-Messung entsprechend Abbildung 17. Die zugehörigen Standardabweichungen betragen (a) 1,7 $\mu$ V, (b) 1,8 $\mu \mathrm{V}$ und (c) $2 \mu \mathrm{V}$ bei 20 ms Mittelungszeit. Abbildung aus Ref. [36].

Das experimentelle Auflösungsvermögen wurde in den markierten Bereichen fern der Stufenkanten ohne den störenden Einfluss der Thermospannungsoszillationen bestimmt. Die zu diesen Bereichen gehörenden Histogramme zeigt Abbildung 18. Bei einer Mittelungszeit von 20 ms beträgt das experimentelle Auflösungsvermögen damit $1.8 \mu \mathrm{V}^{15}$

15 Im Rahmen einer vorangegangen Dissertation von K. Engel wurde ein Auflösungsvermögen von $0.7 \mu \mathrm{V}$ bei einer Mittelungszeit von $40 \mathrm{~ms}$ erreicht $\left(\mathrm{U}_{\text {Bias }}=1 \mathrm{mV}, \mathrm{I}_{\mathrm{t}}=1 \mathrm{nA}\right.$ auf Au(111)@80 K) [30]. Bei 40 ms Mittelungszeit hat sich die jetzige Auflösungsgrenze der STP-Anlage damit von $0,7 \mu \mathrm{V} z u$ 1,3 $\mu \mathrm{V}$ geringfügig verschlechtert. Jedoch ist der Mehrwert, Ladungstransportexperimente durchführen zu können, hervorzuheben. 


\section{Die $\operatorname{Si}(111)(\sqrt{3} \times \sqrt{ } 3)-A g$ Rekonstruktion}

Die strukturellen und elektronischen Eigenschaften der $\operatorname{Si}(111)(\sqrt{3} x \sqrt{3})-A g$ Rekonstruktion werden seit Jahrzehnten kontrovers diskutiert. Trotz einer Vielzahl von Analysemethoden, wie z.B. Photoemission- und inverse Photoemissionsstudien [61-65], „First Principle“ Berechnungen [66, 67], STM-Untersuchungen [68-74], optischer Spektroskopie [75] und Analysen der Oberflächenleitfähigkeit [63, 76-79], war bis Ende der 90er Jahre z. B. noch umstritten, ob diese Oberfläche metallisch oder halbleitend ist. Einen Überblick zum aktuellen Stand der Forschung gibt das nachfolgende Kapitel. Im Anschluss daran werden die einzelnen Prozessschritte zur Präparation der $\operatorname{Si}(111)(\sqrt{3} \mathrm{x} \sqrt{3})-A g$ Rekonstruktion, wie sie in dieser Arbeit verwendet worden sind, vorgestellt. Eine detaillierte Charakterisierung der elektronischen und strukturellen Eigenschaften der hergestellten Proben schließt dieses Kapitel ab.

\subsection{Stand der Forschung}

\subsubsection{Strukturelle Eigenschaften}

Noch zu Beginn dieser Arbeit wurden zwei unterschiedliche Strukturmodelle der $\operatorname{Si}(111)(\sqrt{3} \times \sqrt{3})-A g$ Rekonstruktion diskutiert. Während das „honeycomb-chained-trimer, $\mathrm{HCT}^{\prime}$ Modell eine symmetrische Einheitszelle entlang der [112]]-Richtung postuliert [66, 67, 72, 80], kommt es nach dem ,inequivalent-trimer, IET“ Modell zu einem Symmetriebruch entlang dieser Richtung [81-84]. Beide Strukturmodelle besitzen identische Bindungspartner, unterscheiden sich aber in ihren geometrischen und damit elektronischen Eigenschaften. Abbildung 19 zeigt die entsprechenden Strukturmodelle. Bei beiden Modellen bestehen die Rekonstruktionen aus je einem Ag-Atom pro unrekonstruierter Si(111)-Einheitszelle. Die AgAtome werden über eine Bindung an die darunterliegenden Si-Atome gebunden. Diese SiLage ist in Trimeren angeordnet. Von jedem Si-Atom gehen vier Bindungen aus. Zwei SiBindungsparter liegen in der Ebene, ein Si-Bindungspartner befindet sich eine Lage tiefer und die verbleibende Bindung wird zum Ag-Atom der Rekonstruktion geknüpft. Der Si-Kristall ist bis hinunter zur vierten Si-Lage leicht verspannt [80]. Abbildung 19(a) zeigt eine Aufsicht der Einheitszelle im HCT-Modell und in (b) die entsprechende Seitenansicht. Die [11 $\overline{2}]$-Richtung ist eine Symmetrieachse und beide Ag-Trimere der Einheitszelle sind äquivalent (in gelb hervorgehoben). Beide Strukturmodelle (HCT und IET) besitzen eine dreizählige Rotations- 
symmetrie gegenüber dem Si-Volumenkristall. Aufgrund dieser Rotationssymmetrie gibt es gemäß dem HCT-Modell Antiphasengrenzen in der Rekonstruktion.

a)

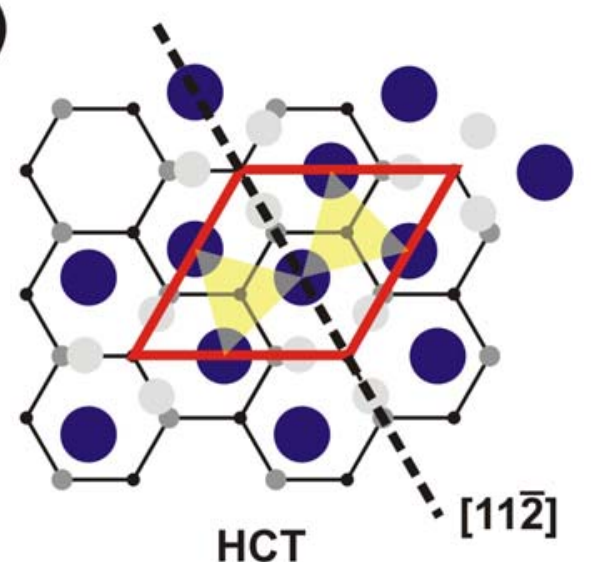

c)

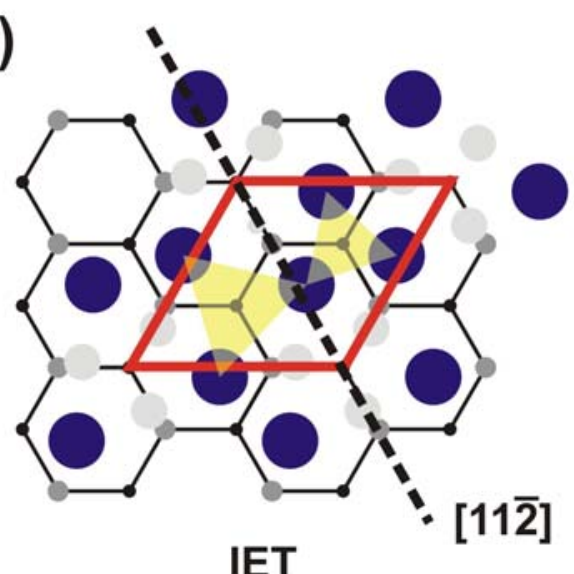

b)

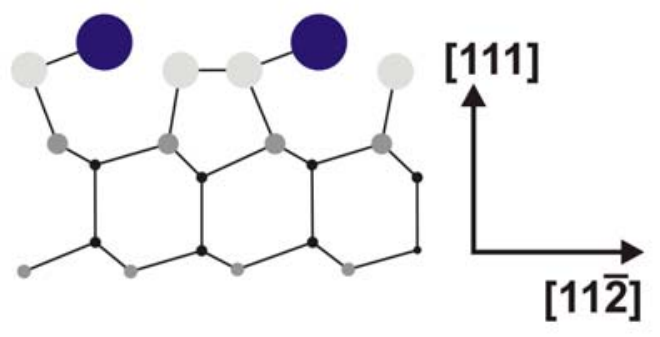

Seitenansicht

d)

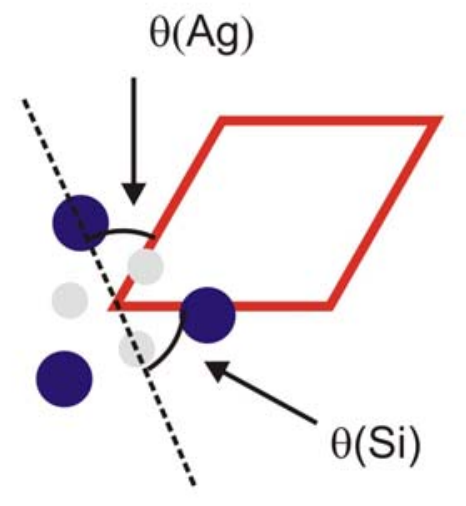

\section{Ag Si (1. Lage) • Si (2. Lage) • Si (3. Lage)}

Abbildung 19: (a) Schematische Darstellung des HCT-Modells und (c) des IET-Modells der $\operatorname{Si}(111)(\sqrt{3} x \sqrt{3})-A g$ Rekonstruktion. Die Einheitszelle ist jeweils rot dargestellt. Sie ist rautenförmig und besitzt eine Kantenlänge von 6,65 А [80, 85]. Ag-Trimere sind gelb schraffiert. (b) Illustriert die Seitenansicht des HCT-Modells. In (d) ist die Definition der Winkel $\theta_{\text {Ag }}$ und $\theta_{\text {Si }}$ gegeben. Abbildung aus Ref. [36].

Im Gegensatz dazu sind beim IET-Modell die Ag-Trimere gegenüber den Si-Trimeren in der (111)-Ebene um $\pm 6^{\circ}$ verdreht und die beiden Ag-Trimere der Einheitszelle besitzen unterschiedliche Größen. Abbildung 19(c) zeigt die entsprechende Einheitszelle im IETModell und (d) illustriert die Definition des Rotationswinkels. Die Symmetrie entlang der [112] $]$-Richtung verschwindet und beide Ag-Trimere der Einheitszelle werden inequivalent. 
Beide Rotationen um $\pm 6^{\circ}$ sind gleichwertig, so dass es zur Ausbildung von zwei Domänen kommt. Die IET ${ }^{+}$-Domäne wird durch $\theta_{\mathrm{Ag}}=66^{\circ}, \theta_{\mathrm{Si}}=54^{\circ}$ charakterisiert und die IET-Domäne entsprechend durch $\theta_{\mathrm{Ag}}=54^{\circ}, \theta_{\mathrm{Si}}=66^{\circ}$. In der IET-Struktur existieren somit zwei unterschiedliche Typen von Domänengrenzen: Antiphasengrenzen aufgrund der Rotationssymmetrie zum Si-Substrat und Zwillingskorngrenzen aufgrund der $\pm 6^{\circ}$ Rotation. Durch TieftemperaturSTM-Untersuchungen bei $62 \mathrm{~K}$ und $6 \mathrm{~K}$ konnten beide Typen von Domänen experimentell bestätigt werden [83, 86, 87]. Ebenso konnten die asymmetrischen Oberflächeneinheitszellen der IET $^{+}$- und IET--Struktur zweifelsfrei bei tiefen Temperaturen abgebildet werden [83, 86].

Das IET-Modell galt daher zu Beginn dieser Arbeit als das allgemein akzeptierte Strukturmodell bei tiefen Temperaturen. Es wurde angenommen, dass das symmetrische STM-Bild der HCT-Struktur bei Raumtemperatur über die Mittelung thermisch induzierter, schnell schwankender IET-Domänen zustande kommt [83]. Bei tiefen Temperaturen (kleiner als $70 \mathrm{~K}$ ) sollten die Schwankungen der IET-Domänen einfrieren und dadurch mit dem STM abbildbar werden [83, 88]. Dieser Interpretation folgend, würde der Unterschied in der Gesamtenergie beider Rekonstruktionen in der Größenordnung von $\sim 20 \mathrm{meV}$ pro Einheitszelle liegen.

\subsubsection{Elektronische Eigenschaften}

Einhergehend zur kontroversen Diskussion über die strukturellen Eigenschaften der $\operatorname{Si}(111)(\sqrt{3} \times \sqrt{3})-A g$ Rekonstruktion, wurden die elektronischen Eigenschaften dieses Systems diskutiert [86]. Mittlerweile sind die elektronischen Eigenschaften dieses Systems jedoch gut verstanden und werden für die IET-Struktur in diesem Kapitel dargestellt.

Abbildung 20(a) zeigt eine winkelaufgelöste Photoelektronenspektroskopie im UV-Spektralbereich (engl. ARUPS) der relevanten Oberflächenzustände bei $E_{f}$ und (b) eine entsprechende DFT-Berechnung. 


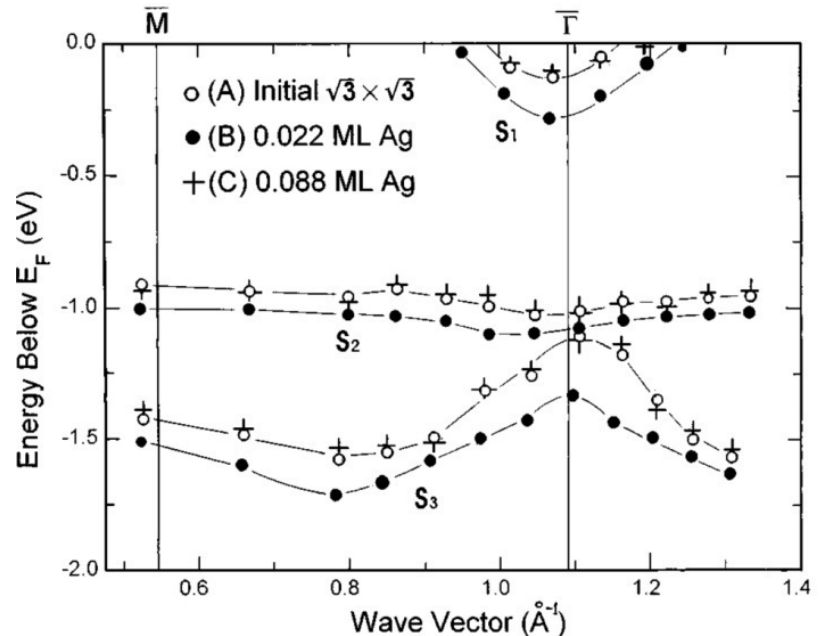

(a)

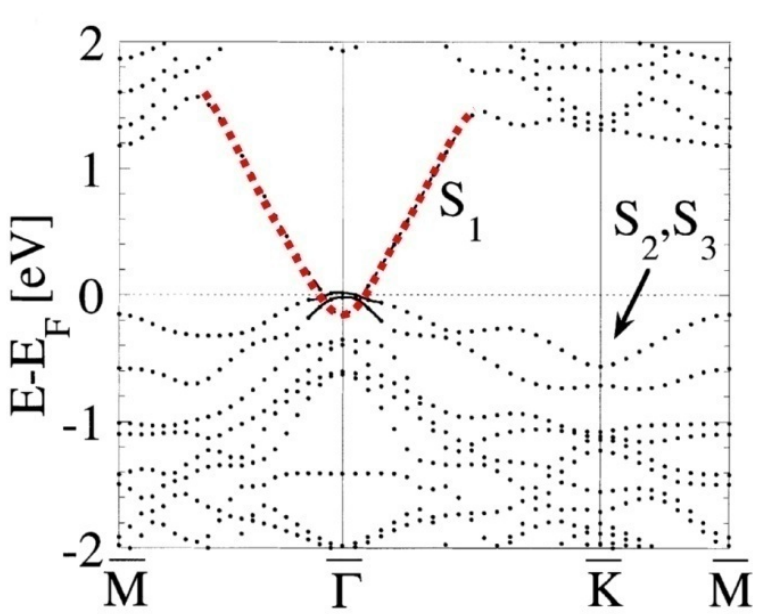

(b)

Abbildung 20: (a) Oberflächenzustände der Si(111) $(\sqrt{3} x \sqrt{3})$-Ag Oberfläche gemessen mit ARUPS [63]. Die Oberfläche wurde bei drei verschiedenen Konzentrationen an überschüssigem Silber vermessen. (b) zeigt eine DFT-Berechnung der Bandstruktur für das IET-Modell aus Ref. [86] mit einem überschüssigem Ag-Adatom pro Einheitszelle. Das $S_{1}$-Band (rot gestrichelte Linie) erstreckt sich über die gesamte Bandlücke und hat sein Energieminimum knapp unterhalb der Fermienergie.

Ein leitungsbandartiger Oberflächenzustand $S_{1}$ und zwei valenzbandartige Zustände $S_{2}$ und $S_{3}$ werden beobachtet. Der Oberflächenzustand $S_{1}$ erscheint parabolisch und ist teilweise gefüllt. Elektronen können in diesem Zustand demnach wie freie ebene Wellen propagieren, so dass sie sich wie ein zweidimensionales freies Elektronengas (2DEG) verhalten. Um den Einfluss zusätzlicher Defekte auf der idealen Rekonstruktion zu demonstrieren, zeigt Abbildung 20 (a) experimentelle Ergebnisse für verschiedene Konzentrationen an zusätzlichem, überschüssigem Silber. Einhergehend mit der zunehmenden Dichte an überschüssigem Silber auf der $\operatorname{Si}(111)(\sqrt{3} \times \sqrt{3})-A g$ Rekonstruktion sinkt das Energieminimum des $\mathrm{S}_{1}$ Zustands relativ zu $\mathrm{E}_{\mathrm{f}}$ ab [89-91]. Die Besetzung des $\mathrm{S}_{1}$-Zustands korrespondiert folglich mit der Ag-Adatom Konzentration. Abbildung 21 zeigt hierzu eine systematische Analyse. 


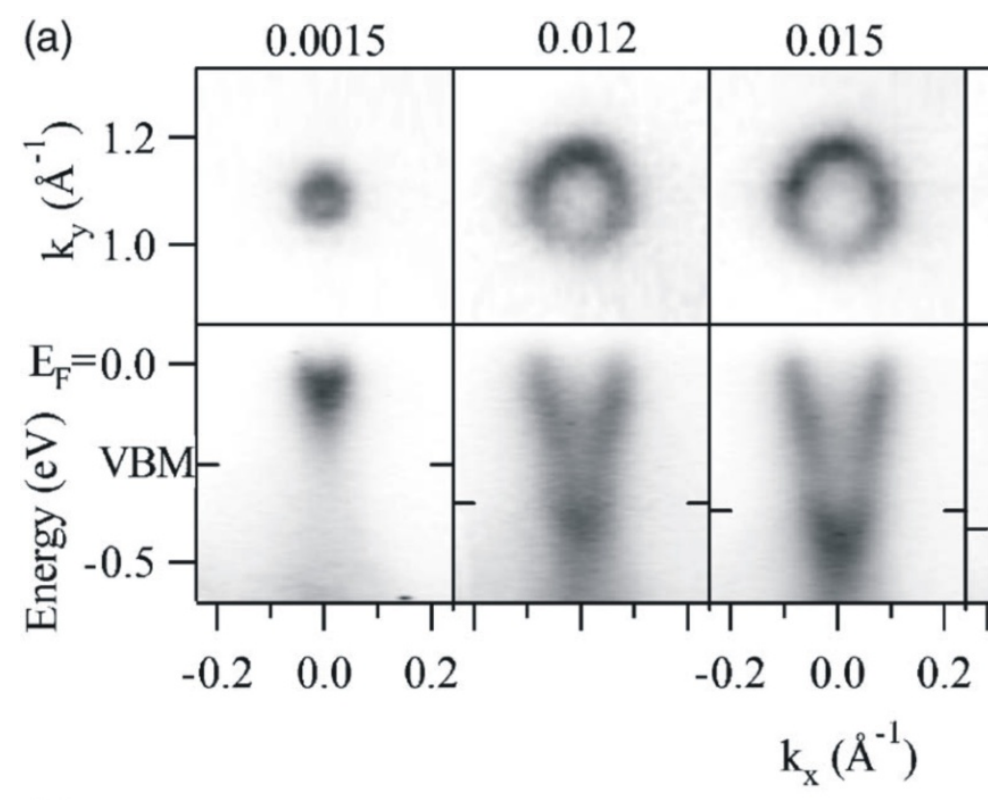

(b)

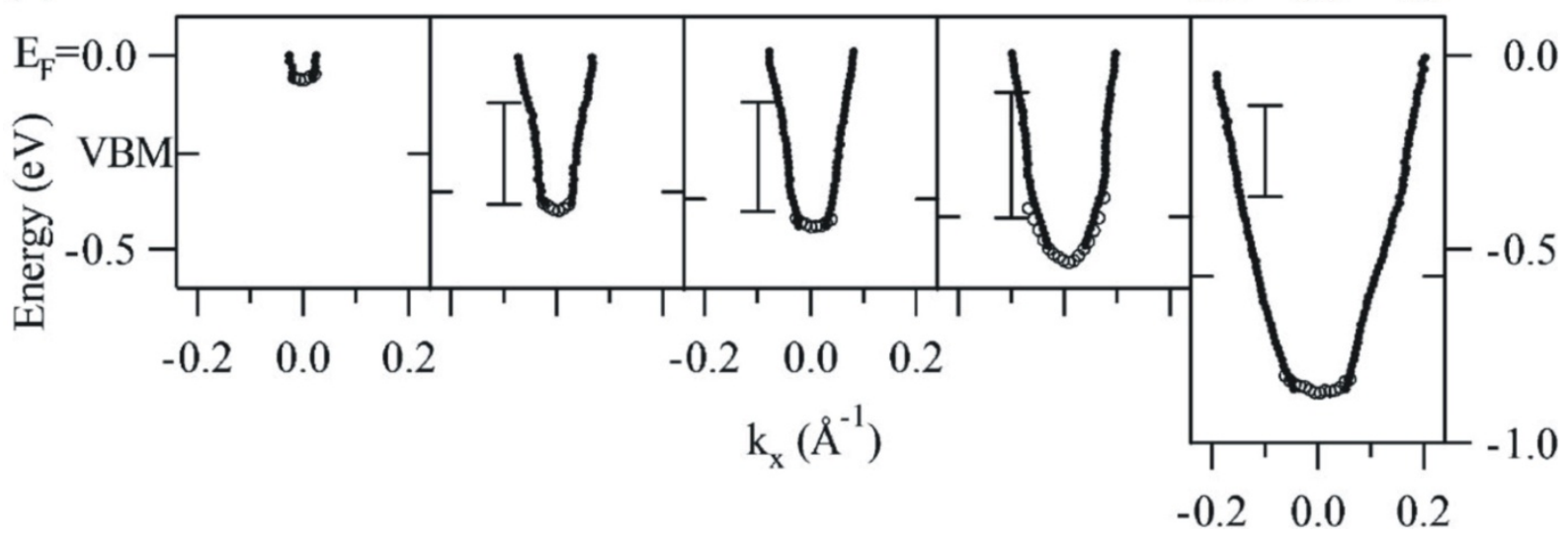

Abbildung 21: (a) Photoemissionsdaten der Fermioberfläche und Banddispersion entlang

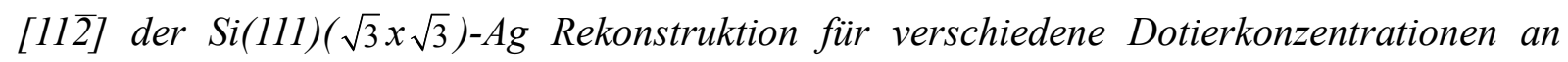
zusätzlichem Silber-Adatomen (0.0015, 0.012, 0.015, 0.022, und 0.086 zusätzlich eingebrachte Elektronen pro Si(111)-1×1-Einheitszelle). (b) Aus (a) gewonnene Banddispersion: Mit zunehmender Ag-Adatom Konzentration rutscht das $S_{1}$-Band unter das Valenzbandmaximum (VBM). Als Substrat wurde n-dotiertes Si verwendet. Abbildung aus Ref. [90]. ${ }^{16}$

Die Ag-Adatom Konzentration wurde von 0.0015 bis 0.086 Ag-Adatome pro $\mathrm{Si}(111)-1 \mathrm{x} 1$ Einheitszelle erhöht. Deutlich ist die isotrope Fermifläche als Ring in der $k_{x}-k_{y}$ Ebene bei $E_{f}$ zu sehen. (b) zeigt die nahezu parabolische Dispersion des $S_{1}$-Bandes bis $E_{f}$. Durch

\footnotetext{
${ }^{16}$ Die Beschriftung der Autoren von „e-/atom“ ist irreführend und im Text wird richtigerweise von Elektronen pro 1x1-Einheitszelle gesprochen.
} 
Adsorption von überschüssigem Silber erhöht sich die Fermienergie von $\sim 100 \mathrm{meV}$ bei geringer Bedeckung auf bis zu $\sim 750 \mathrm{meV}$.

Durch derartige Messungen konnte zweifelsfrei belegt werden, dass Ag-Adatome als Donatoren für den Oberflächenzustand $S_{1}$ fungieren. Durch eine Vielzahl von weiteren Messungen wurde nachgewiesen, dass dabei jedes Ag-Adatom im Mittel ein Elektron in den $\mathrm{S}_{1}$-Zustand doniert [63, 86, 89-95]. Es gibt zudem Hinweise darauf, dass sich die Dispersion des Oberflächenzustands $S_{1}$ mit der Dotierung leicht verändert. Bei hoher Dotierung soll demnach eine stärkere Abweichung von einer parabolischen Dispersion stattfinden [90]. Die effektive Masse der Elektronen im $\mathrm{S}_{1}$-Zustand beträgt bei geringer bis mittlerer Dotierung $\left(\mathrm{E}_{\mathrm{f}}<400 \mathrm{meV}\right) 0.13 \mathrm{~m}_{\mathrm{e}}[87,89,90,92,96]$.

Die Abbildung 21(b) zeigt zusätzlich die Lage der Si-Oberflächenvalenzbandkante (OVBM) bei verschiedenen Ag-Dotierungen auf n-dotiertem Silizium. Bei geringer Ag-Dotierung liegt das Minimum des $\mathrm{S}_{1}$ Zustands $\left(\mathrm{E}_{0}\right) \sim 100 \mathrm{meV}$ über dem OVBM. Bei hoher Dotierung $(>0.0015 \mathrm{Ag}$ pro $\mathrm{Si}(111)-1 \times 1)$ sinkt $\mathrm{E}_{0}$ unter das OVBM. Die Lage von $\mathrm{E}_{0}$ als Funktion der Dotierung fasst Abbildung 22(a) zusammen.

Als Folge freier Zustände in der Bandlücke treten Elektronen aus dem Halbleiter in den Oberflächenzustand $S_{1}$ über und seine hohe Zustandsdichte pinnt das Fermilevel an der Oberfläche. Dabei kommt es im Silizium zur Ausbildung einer positiven Raumladungszone und dementsprechend zu einer positiven Oberflächenbandverbiegung. Die Folge ist eine Verarmungszone, die den Oberflächenzustand elektrisch von der Volumenleitfähigkeit des Siliziums trennt (näheres dazu im Kapitel 3.1). Abbildung 22 (b, c) zeigt schematisch den Verlauf der Oberflächenbandverbiegung bei verschieden Dotierungen des Oberflächenzustands für n-dotiertes Silizium. Mit zunehmender Dotierung durch Ag-Adatome steigt das chemische Potential im Oberflächenzustand. Es stehen damit weniger freie Zustände in der Bandlücke für Elektronen zur Verfügung. Dementsprechend können weniger Elektronen des Halbleiters in den Oberflächenzustand übertreten, woraus eine verringerte Oberflächenbandverbiegung resultiert. 
(a)

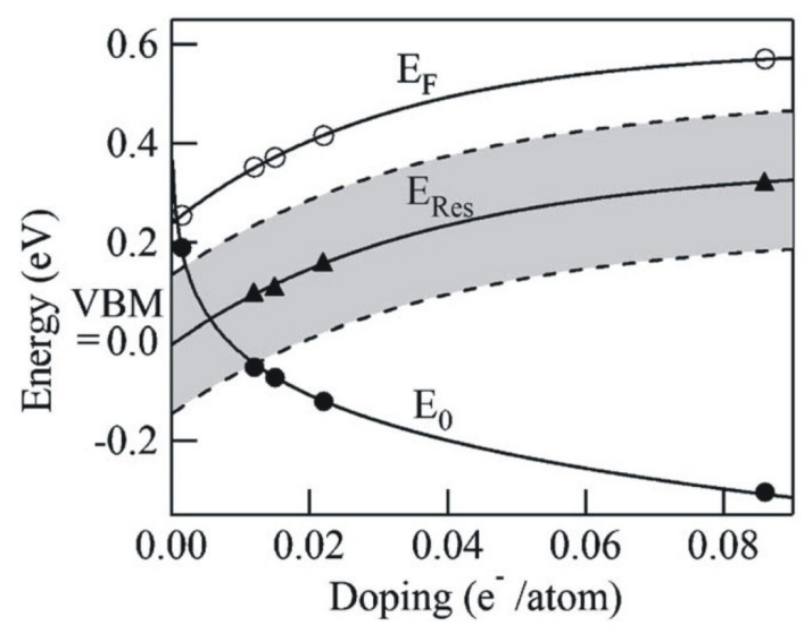

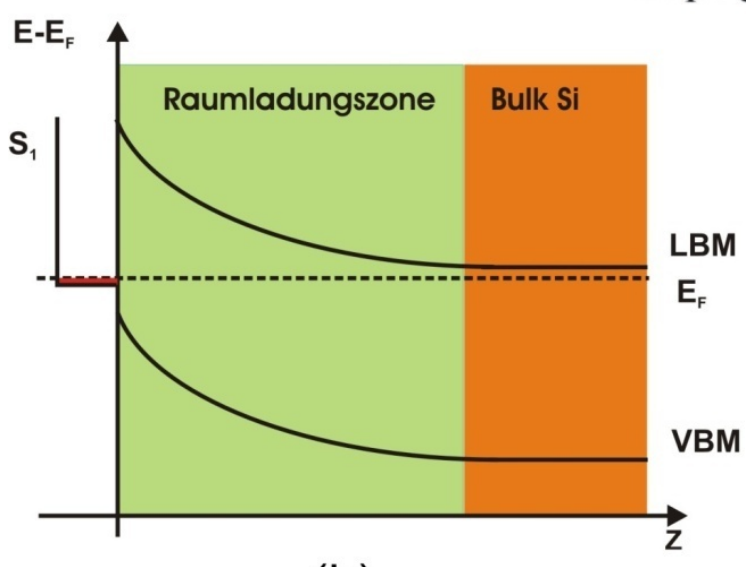

(b)

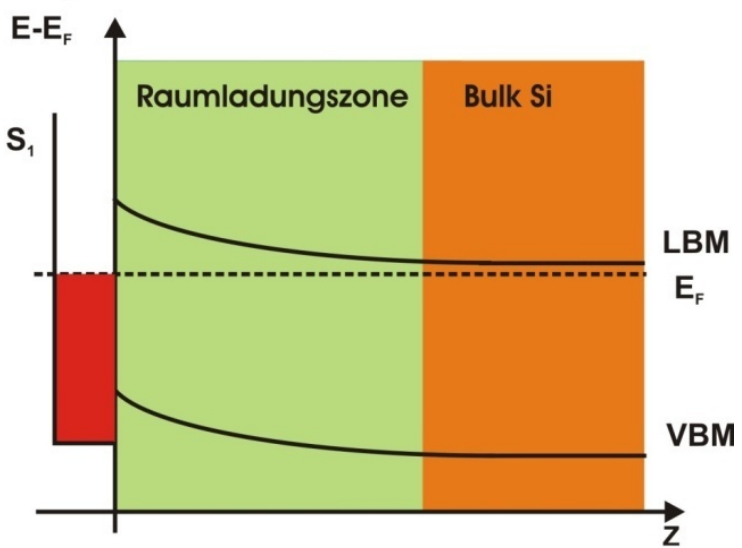

(c)

Abbildung 22: (a) zeigt die Fermienergie $E_{f}$ und das Bandminimum $E_{0}$ des $S_{1}$-Zustands als Funktion der Ag-Adatom Konzentration pro Si(111)-1×1-Einheitszelle auf $n$-dotiertem Silizium (Energie relativ zum OVBM). Das Bandminimum $E_{0}$ schiebt von $0.19 \mathrm{eV}$ über dem OVBM zu $0.30 \mathrm{eV}$ unter das OVBM und zeigt damit eine Abweichung vom „Rigid-BandModel“. E Ees zeigt die energetische Lage des Ag-Defektzustands. Abbildung aus Ref. [90]. $(b, c)$ zeigen schematische Banddiagramme des $S_{1}$-Oberflächenzustands und der Raumladungszone: (b) geringe Dotierung mit $E_{0}>O V B M$ und (c) mit zusätzlicher Dotierung und $E_{0}<O V B M$. Gemäß (a) verkleinert sich der Bereich der Raumladungszone mit steigender Dotierung. Abbildung aus Ref. [36]. 


\subsection{Präparation der Si(111)( $\sqrt{3} \times \sqrt{3})$-Ag Rekonstruktion}

Die Si(111) $(\sqrt{3} \mathrm{x} \sqrt{ } 3)$-Ag Rekonstruktion wurde auf Si(111)-Wafern (n-Typ: P) mit einer Dicke von $180 \mu \mathrm{m}$, einem spez. Widerstand von (93...130) $\Omega \mathrm{cm}$ und einer Fehlneigung kleiner als $0.5^{\circ}$ präpariert. Zunächst wurden Probenstücke mit einer Größe von $2 \times 8 \mathrm{~mm}^{2}$ mit einer Diamantgravurnadel aus dem Wafer heraus präpariert. Die lange Probenseite entspricht der

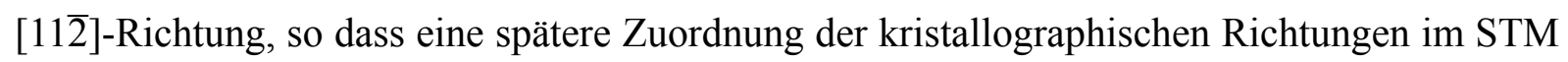
stets zweifelsfrei geschehen konnte. Abbildung 23 zeigt den eingebauten Wafer in dem fertig montierten Probenhalter, welcher im Rahmen der vorangegangenen Diplomarbeit entwickelt und dort ausführlich beschrieben worden ist [33]. Der Wafer wird auf beiden Seiten durch dünne, federnde Tantal-Bleche auf jeweils $1 \mathrm{~mm}$ Länge kontaktiert und mechanisch fixiert, so

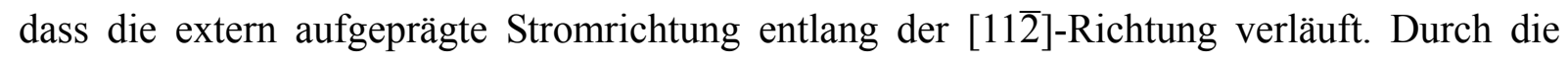
„schwimmende Lagerung“ sollen Spannungen im Wafer während der späteren Probenpräparation vermieden werden. Oxidschichten auf den Metallen und auf dem Wafer erhöhen den Gesamtwidertand der Probe in diesem Zustand auf mehrere $\mathrm{M} \Omega$.

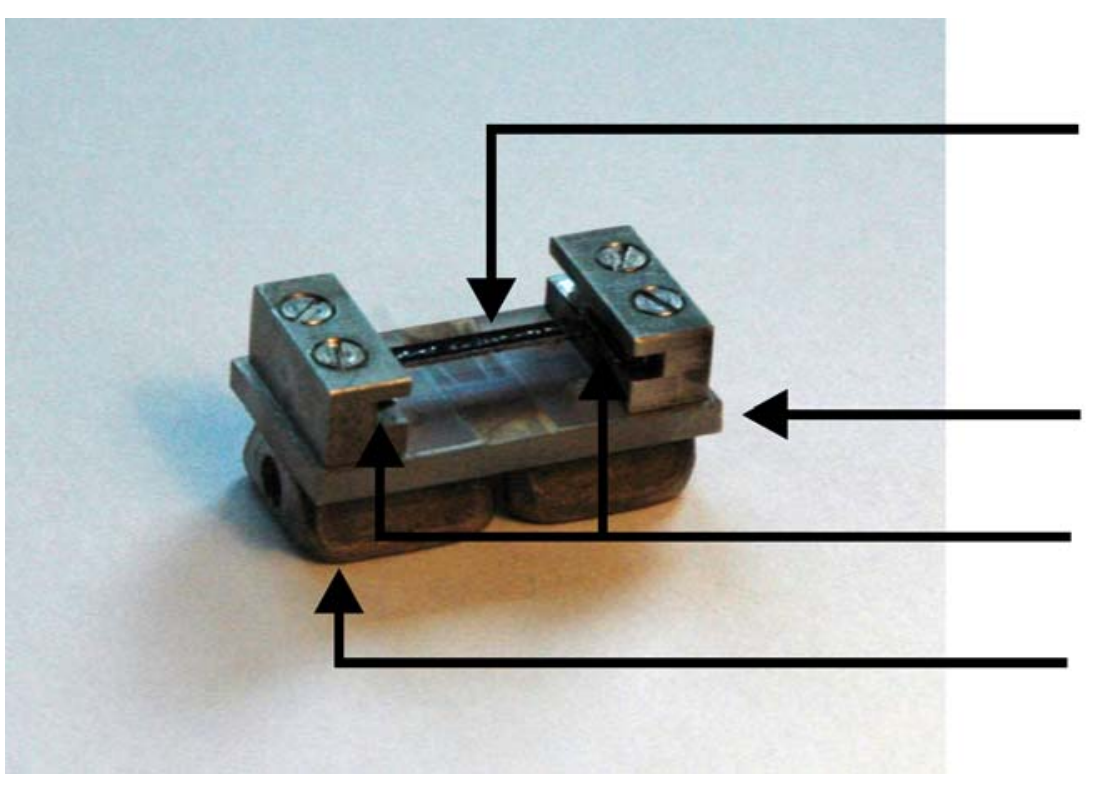

\section{Si(111)-Wafer}

Saphirplatte

Tantal-Bleche

Edelstahlsegment

Abbildung 23: Zweifach segmentierter Probenhalter mit eingebautem Si(111)-Wafer. Die elektrische Kontaktierung sowie mechanische Halterung des Wafers geschieht über dünne Tantal-Bleche.

Die Präparation der Si(111) $(\sqrt{3} x \sqrt{3})$-Ag Rekonstruktion geschieht unter UHV-Bedingungen bei einem Basisdruck von $5 \cdot 10^{-11} \mathrm{mBar}$ in einem zweistufigen Verfahren $[71,72,80]$. In einem ersten Schritt wird zunächst eine möglichst defektfreie Si(111)-7x7 Oberfläche 
präpariert. In einem zweiten Schritt wird darauf Ag deponiert und die Oberfläche wird durch eine kurze Temperaturbehandlung in die $\operatorname{Si}(111)(\sqrt{3} \mathrm{x} \sqrt{ } 3)-A g$ Rekonstruktion überführt. Der Wafer wurde resistiv über die Kontakte, die auch der späteren Kontaktierung dienen, geheizt. Es wird angenommen, dass hierbei Tantal in den Si-Wafer hinein diffundiert und es zur Ausbildung eines ohmschen Kontakts kommt (siehe hierzu auch Kapitel 2.3.1). Die Temperaturmessung geschah durch ein Pyrometer bei Annahme einer konstanten Emissivität von $70 \%{ }^{17}$

Die Prozessschritte im Detail sind:

1. Präparation einer möglichst defektfreien Si(111)-7x7 Oberfläche:

Der frisch eingebaute Wafer wird im UHV für mehrere Stunden resistiv auf $750{ }^{\circ} \mathrm{C}$ erhitzt, um Verunreinigungen auf den Oberflächen zu entfernen. Es hat sich gezeigt, dass hierbei viele Verunreinigungen der metallischen Komponenten (Edelstahl und Tantal) ebenfalls desorbieren. Anschließend wird der Wafer für wenige Sekunden auf $1250{ }^{\circ} \mathrm{C}$ erhitzt. Bei diesem Vorgang desorbieren auch die restlichen Verunreinigungen von der Oberfläche, was sich in einem starken Druckanstieg bemerkbar macht. Nach mehrmaligem Erhitzen auf $1250^{\circ} \mathrm{C}$ bleibt ein Druckanstieg nahezu völlig aus und der Wafer wird langsam wieder auf Raumtemperatur abgekühlt. Der Widerstand zwischen den Kontakten liegt nach diesen Prozessschritten typischerweise bei $100 \mathrm{k} \Omega$.

2. Präparation der $\operatorname{Si}(111)(\sqrt{ } 3 x \sqrt{ } 3)-A g$ Rekonstruktion:

Bei $450{ }^{\circ} \mathrm{C}$ wird die Si(111)-7x7 Oberfläche mit einem Ag-Fluss von $1 \AA / \mathrm{min}^{18}$ bedampft und anschließend noch 10 weitere Minuten bei gleichbleibender Temperatur getempert. Während des Temperns desorbiert ein Großteil des überschüssigen Silbers, welches nicht zur Bildung der $\operatorname{Si}(111)(\sqrt{3} \times \sqrt{3})$-Ag Rekonstruktion benötigt wird (Details siehe Kapitel 2).

Die einzelnen Präparationsschritte wurden mit „Low Energy Electron Diffraction“ (LEED) und STM-Messungen überprüft. Abbildung 24 (a) zeigt beispielhaft eine LEED-Messung auf der frisch präparierten $\operatorname{Si}(111)-7 \times 7$ Oberfläche bei 50,6 eV. Die hellen 1x1 Maxima

\footnotetext{
${ }^{17}$ Modell IGA 140 der Firma IMPAC Infrared GmbH

${ }^{18}$ Zur Eichung wurden dicke Ag(111)-Filme auf Glimmer verwendet, welche bei Raumtemperatur deponiert worden sind.
} 
entsprechen den reziproken Gittervektoren $\mathrm{g}_{\mathrm{Si}}$ und sind hexagonal angeordnet. Im Abstand $1 / 7 \cdot \mathrm{g}_{\mathrm{Si}}$ - entsprechend der Einheitszellengröße im Ortsraum - erkennt man die reziproken Gittervektoren der $\operatorname{Si}(111)-7 \times 7$ Oberfläche. (b) zeigt eine LEED-Messung der frisch präparierten $\operatorname{Si}(111)(\sqrt{3} x \sqrt{3})$-Ag Rekonstruktion, ebenfalls bei 50,6 eV. Das Beugungsbild der

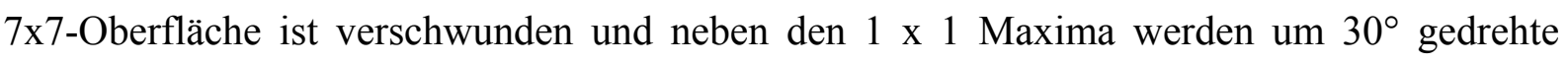
Beugungsreflexe mit einem reziproken Abstand von $1 / \sqrt{ } 3 \cdot \mathrm{g}_{\mathrm{Si}}$ sichtbar. Es gibt weder Hinweise auf verbliebene 7x7-Domänen noch auf überschüssiges Silber, dass sich durch die Reflexe von kristallinem Silber bemerkbar machen müsste.

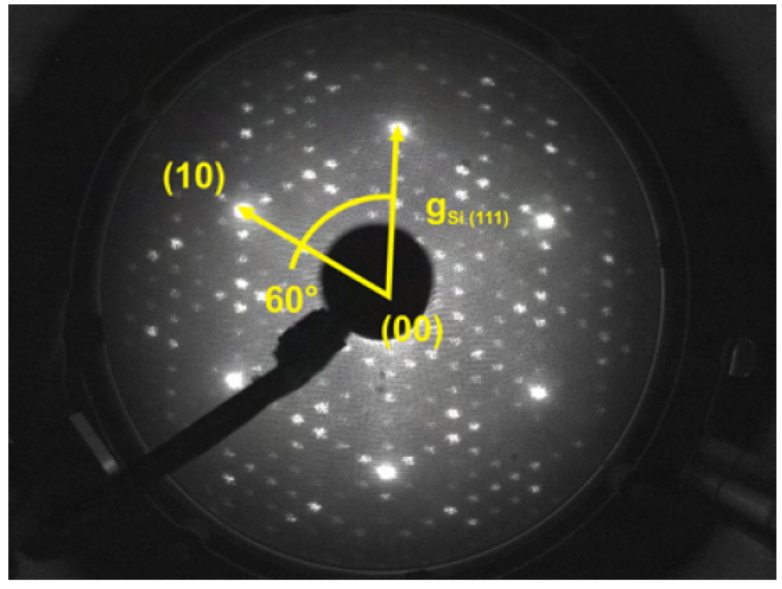

(a)

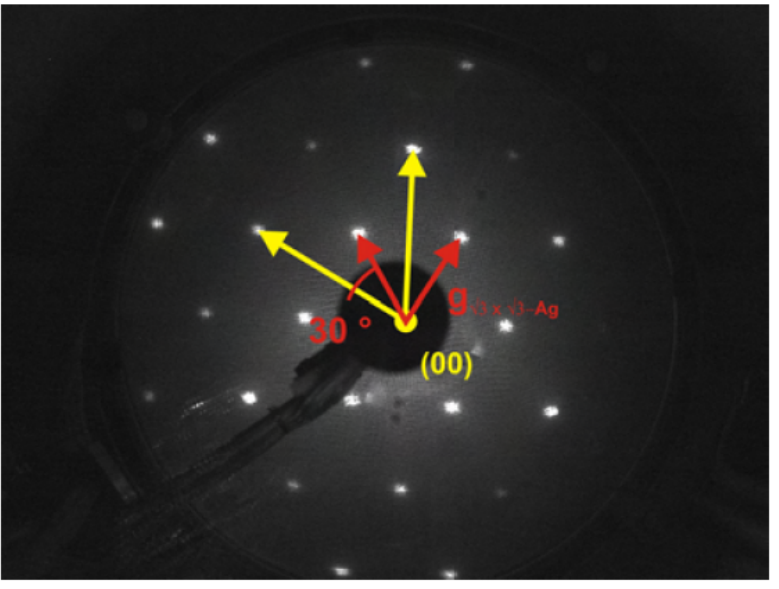

(b)

Abbildung 24: LEED-Messungen (a) auf der Si(111)-7x7 und (b) auf der Si(111)( $\sqrt{ } 3 x \sqrt{ } 3)$ - Ag Rekonstruktion bei jeweils 50,6 eV direkt nach der Präparation. Abbildung aus Ref. [36].

Die lokalen Eigenschaften der Probe können zwar mit LEED nicht analysiert werden, wohl aber mittels STM-Topographien. Abbildung 25 zeigt eine STM-Topographie der frisch präparierten $7 \times 7$-Oberfläche. In einer ansonsten ungestörten 7x7-Rekonstruktion lassen sich viele atomare Defekte finden. Auf eine ausführliche Beschreibung der STM-Daten der 7x7Oberfläche wird an dieser Stelle jedoch verzichtet (siehe hierfür bspw. Ref. [97, 98]).

STM-Topographien nach Präparation der Si(111) $(\sqrt{3} \mathrm{x} \sqrt{ } 3)$-Ag Rekonstruktion zeigt Abbildung 26. Die Defektdichte scheint im Vergleich zu Abbildung 25 wesentlich geringer zu sein und wird auf ca. 11 Defekte auf $400 \mathrm{~nm}^{2}$ abgeschätzt. Die strukturellen und elektronischen Eigenschaften der derart präparierten Proben werden im nächsten Abschnitt im Detail vorgestellt. 


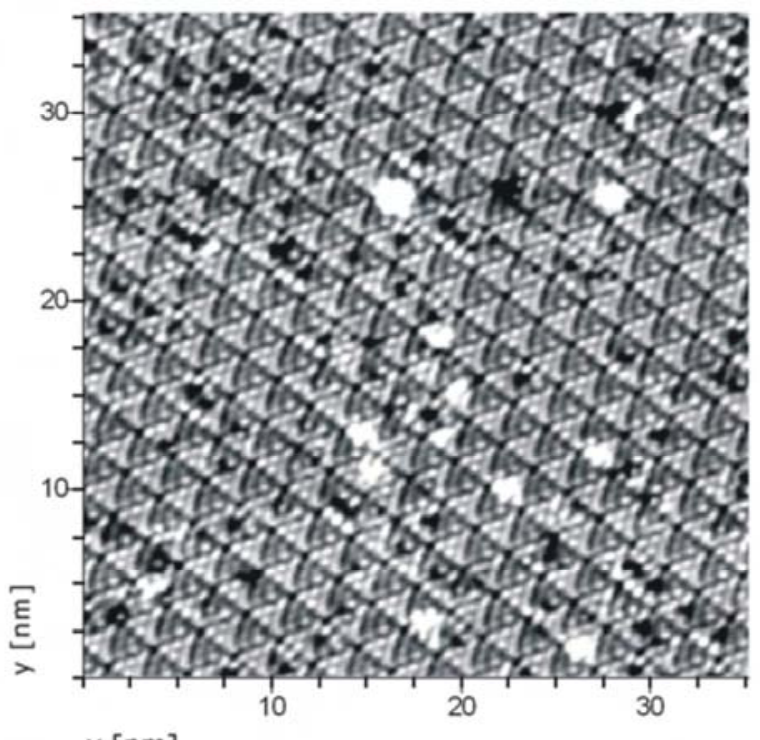

$x[\mathrm{~nm}]$

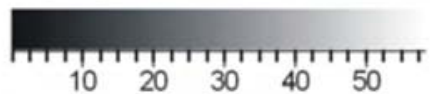

relative Spitzenhöhe [pm]

(a)
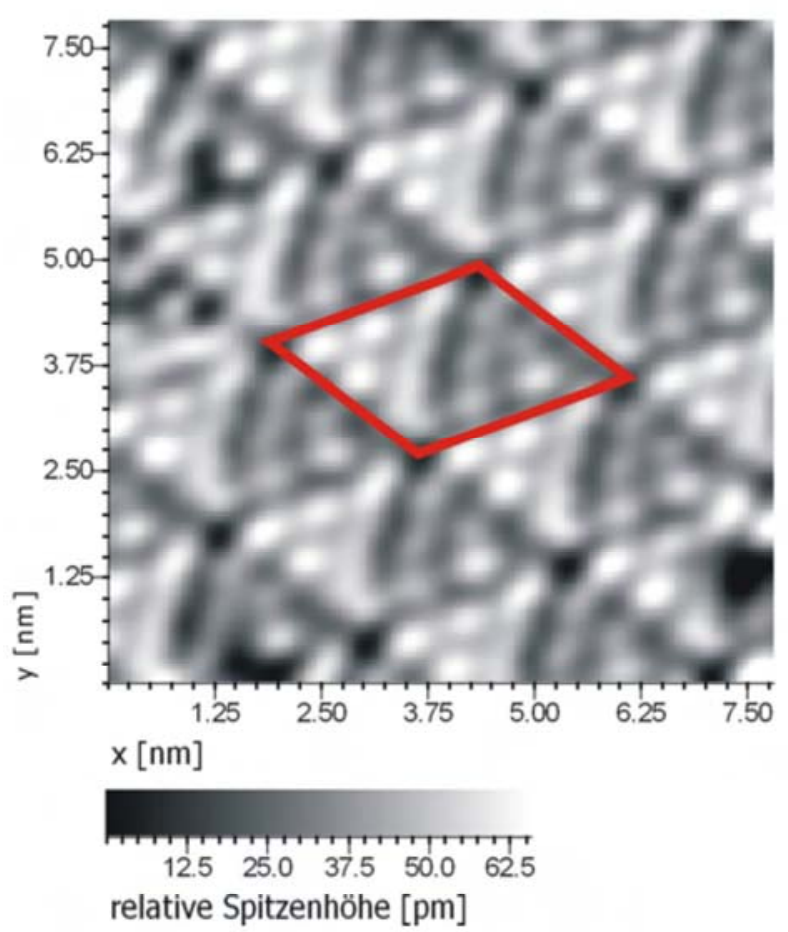

(c)

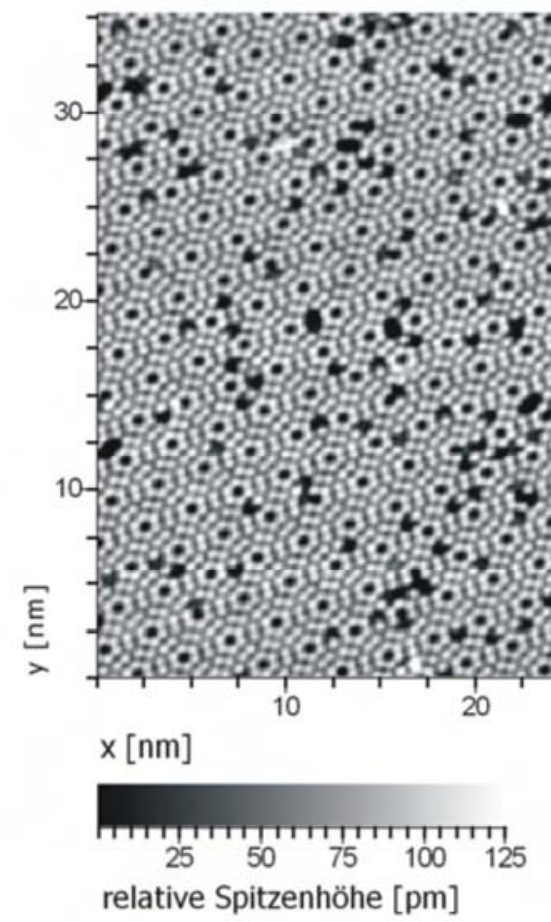

(b)

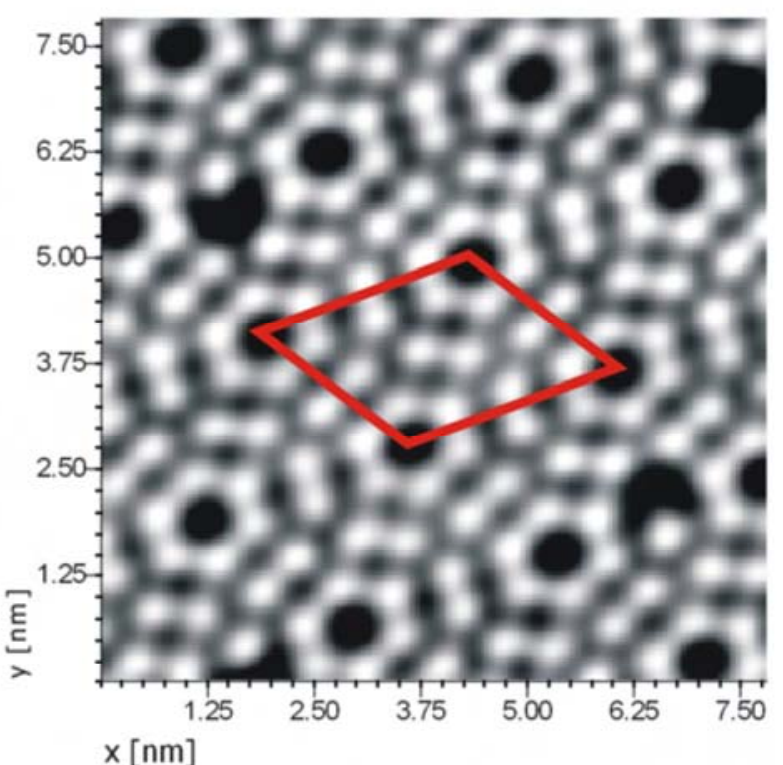

$x[\mathrm{~nm}]$

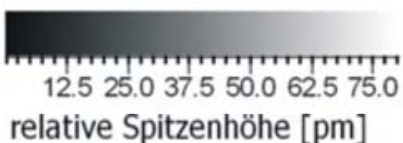

(d)

Abbildung 25: STM-Topographien der frisch präparierten Si(111)-7x7 Oberfläche. (a) zeigt die besetzten Zustände bei $U_{\text {Bias }}=-1,8 \mathrm{~V}$ und $I_{t}=100 \mathrm{pA}$ und (b) die unbesetzten Zustände bei $U_{\text {Bias }}=2$ V und $I_{t}=200 \mathrm{pA}$. (c) und (d) zeigen vergrößerte Ausschnitte und illustrieren die 7x7Einheitszelle. Abbildung aus Ref. [36]. 

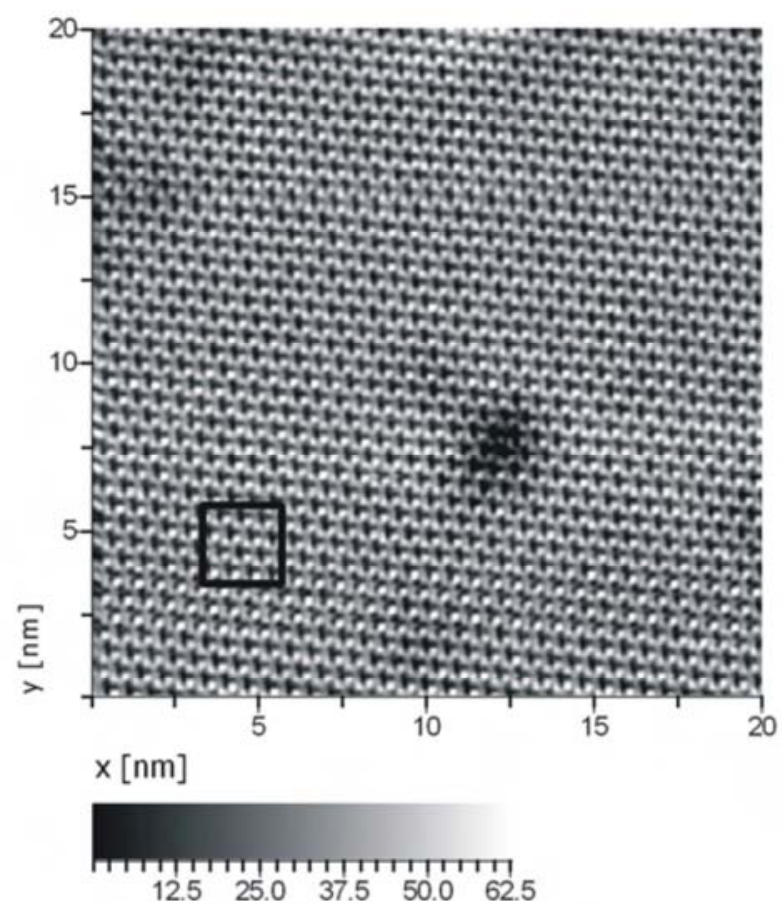

relative Spitzenhöhe $[\mathrm{pm}]$

(a)

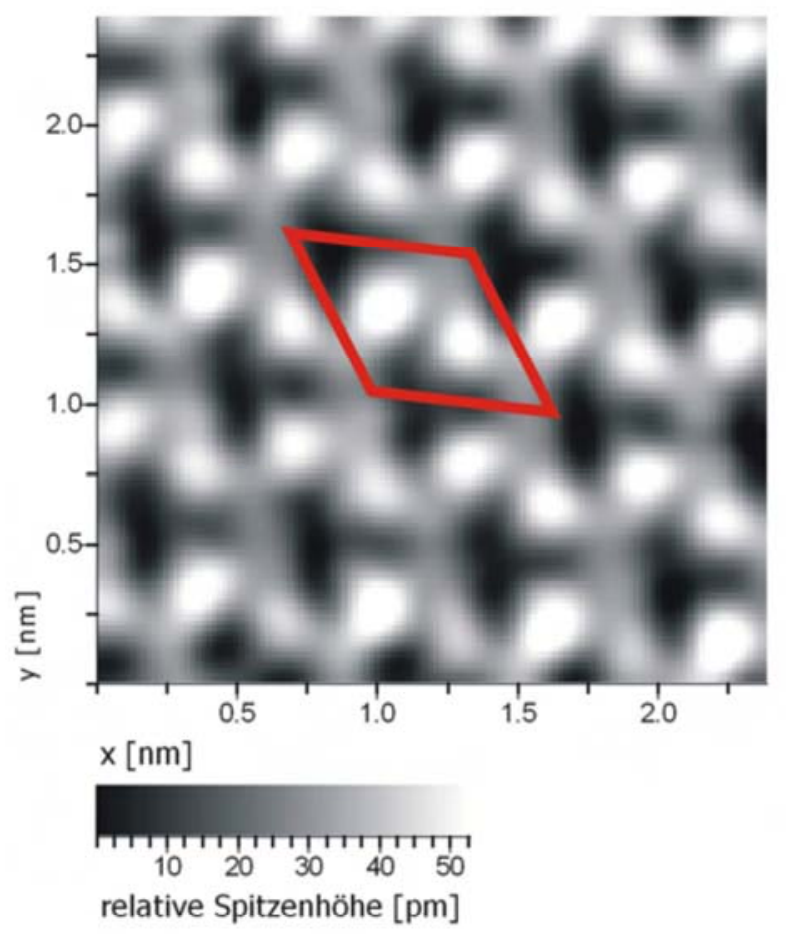

(c)
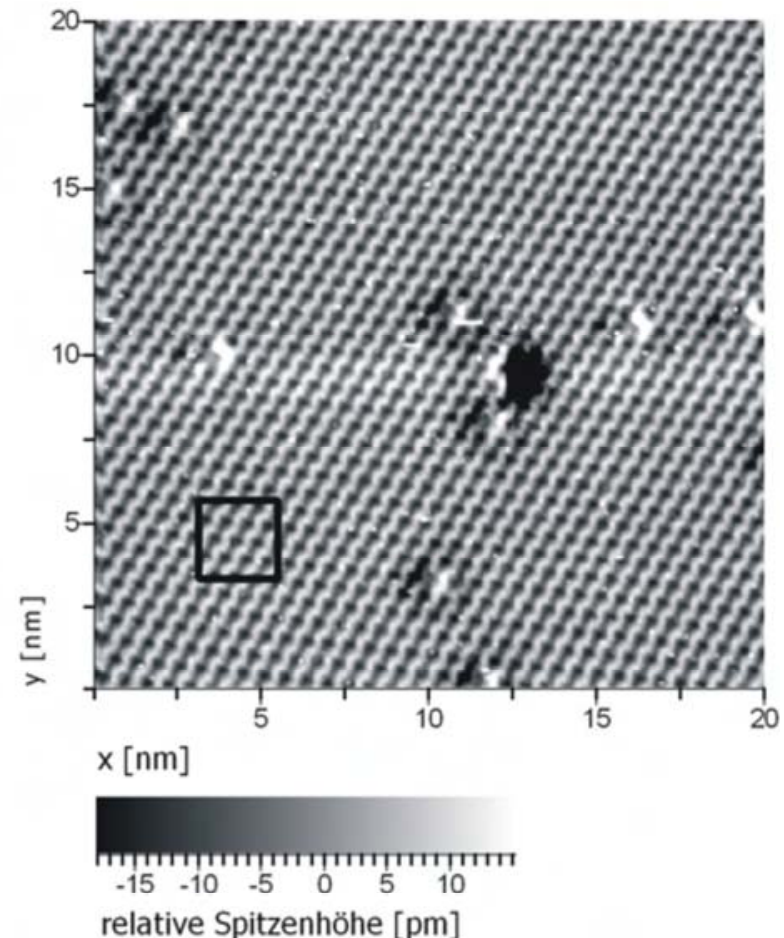

(b)

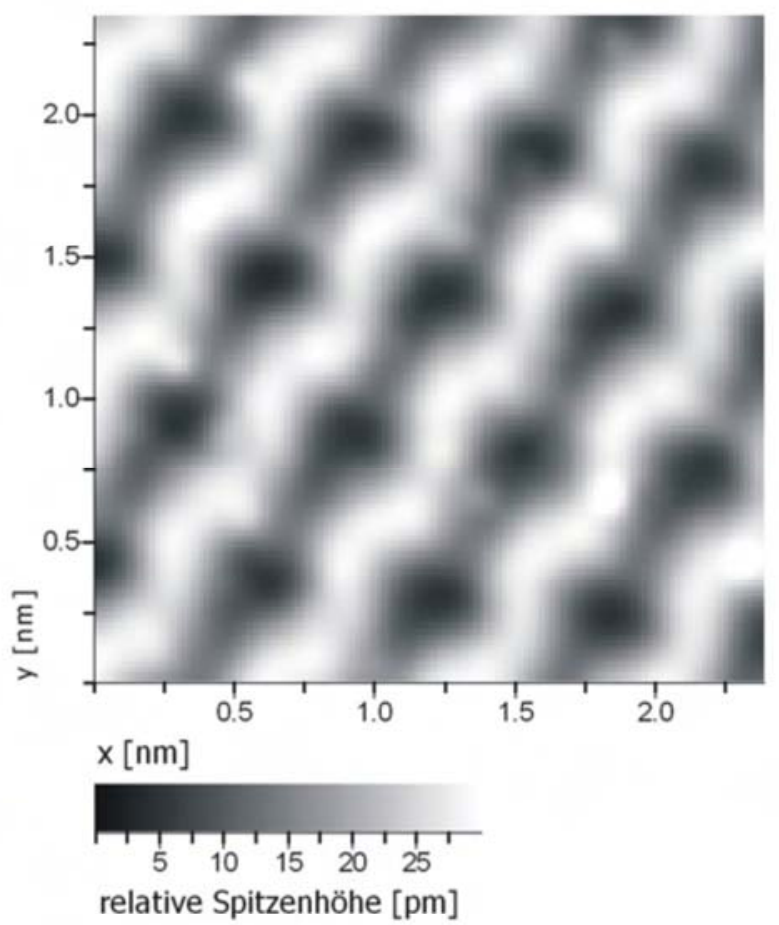

(d)

Abbildung 26: STM-Topographien der frisch präparierten $\operatorname{Si}(111)(\sqrt{ } 3 x \sqrt{ } 3)-A g$ Rekonstruktion. (a) zeigt die besetzten Zustände bei $U_{\text {Bias }}=-50 \mathrm{mV}$ und $I_{t}=3 \mathrm{nA}$. (b) zeigt die unbesetzten Zustände bei $U_{\text {Bias }}=2 \mathrm{~V}$ und $I_{t}=100 \mathrm{pA}$. (c) und (d) zeigen vergrößerte Ausschnitte und illustrieren die Einheitszelle. Abbildung aus Ref. [36]. 


\subsection{Detaillierte Charakterisierung der verwendeten Proben}

Im vorherigen Abschnitt wurde dargestellt, dass sich die Ladungsträgerdichte im 2DEG in einem weiten Bereich verändern lässt. Um intrinsische Transporteigenschaften dieses Systems spezifizieren zu können, ist daher eine genaue Kenntnis der elektronischen und strukturellen Eigenschaften notwendig. Die für diese Arbeit relevanten Proben wurden in situ, direkt vor den Transportmessungen detailliert charakterisiert. Die experimentellen Ergebnisse hierzu, sowie weitere Informationen zum relevanten Probensystem, werden in diesem Abschnitt vorgestellt.

\subsubsection{Elektronische Eigenschaften}

Die Besetzung des $\mathrm{S}_{1}$-Zustands ist der zentrale Parameter mit dem die elektrischen Transporteigenschaften des 2DEG korrelieren. Mit Hilfe von Raster-Tunnel-Spektroskopien (engl. Abkürzung: STS) am Ort der späteren Transportuntersuchungen wurden die elektronischen Eigenschaften daher in situ charakterisiert. Abbildung 27 zeigt eine RasterTunnel-Spektroskopie auf der präparierten $\operatorname{Si}(111)(\sqrt{3} \times \sqrt{3})$-Ag Oberfläche.

Durch Vergleich der STS-Daten mit bisher veröffentlichten Raster-Tunnel-Spektroskopien können die einzelnen spektralen Signaturen plausibel den Oberflächenzuständen $S_{1}$ bis $S_{3}$ zugeordnet werden $[65,83,99]$. Der Oberflächenzustand $S_{1}$ ist besetzt und sein Minimum befindet sich ca. $250 \mathrm{meV}$ unter $\mathrm{E}_{\mathrm{f}}$. Seine Zustandsdichte ist nahezu kastenförmig, wie es für ein zweidimensionales System zu erwarteten ist. Die effektive Masse der Elektronen des $\mathrm{S}_{1}$ Zustands ist bei dieser Besetzung durch eine Vielzahl von Photoemissions-Messungen auf $0.13 \mathrm{~m}_{\mathrm{e}}$ spezifiziert worden, so dass sich die Fermiwellenlänge mit diesen Angaben zu $\lambda_{\mathrm{F}}=68 \AA$ bestimmen lässt $[87,89,90,96]$. 


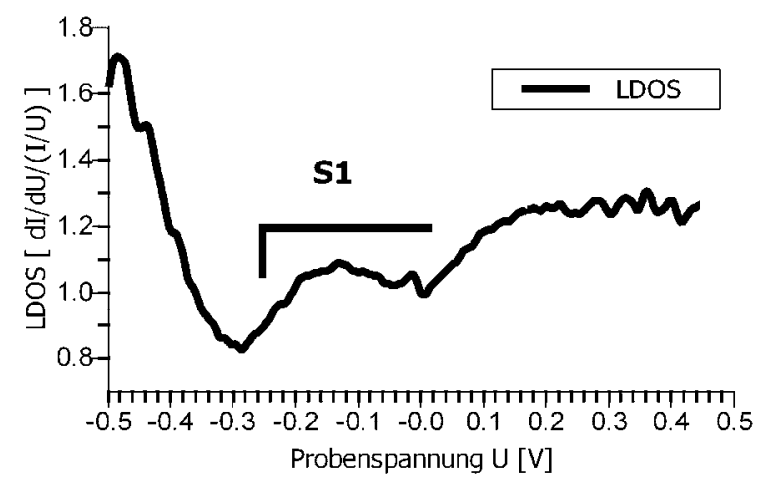

a)

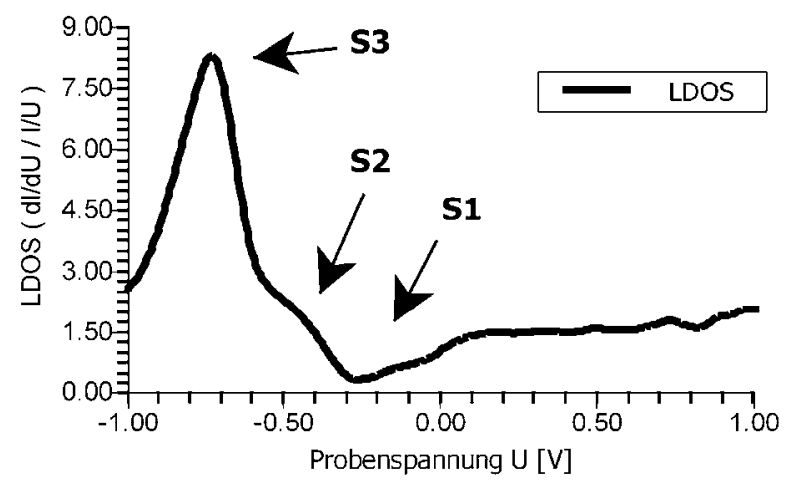

b)

Abbildung 27: Lokale Zustandsdichte (LDOS) der ungestörten $\operatorname{Si}(111)(\sqrt{3} x \sqrt{3})-A g$ Oberfläche (a) im Energieintervall von (-1...1) V bei einer Energieauflösung von $4 \mathrm{mV}$, gemittelt über einen Probenbereich von $40 \mathrm{~nm} \times 40 \mathrm{~nm}$. (b) zeigt STS-Daten im Energieintervall von $(-0,5 \ldots 0,5) \quad V$ bei einer Energieauflösung von $2,5 \mathrm{mV}$, gemittelt über einen Probenbereich von $30 \mathrm{~nm} \times 30 \mathrm{~nm}$. Die Mittelung wurde jeweils auf atomar glatten, defektfreien Probenbereichen ausgeführt. Abbildung aus Ref.[36].

Der Gesamtwiderstand wurde im fertig präparierten Zustand in situ in einer Zweipol-Messung zwischen den Klemm-Kontakten bestimmt. Abbildung 28 zeigt eine gemessene I(U)Kennlinie. Bei beiden Polaritäten wird ein ohmsches Verhalten beobachtet. Der Gesamtwiderstand für negative Querspannung ist größer als bei positiver Querspannung. Der makroskopische Widerstand ergibt sich zu $\mathrm{R}_{\mathrm{U}>0}=(4,62 \pm 0,14) \mathrm{k} \Omega$ und $\mathrm{R}_{\mathrm{U}<0}=(6,69 \pm 0,20) \mathrm{k} \Omega$. Der Fehler wurde abgeschätzt aus der Reproduzierbarkeit der I(U)-Kennlinie bei mehrmaligem Messen. Die Asymmetrie der I(U)-Kennlinien wird auf Eigenschaften der Metall-Halbleiter-Kontakte zurückgeführt, welche offensichtlich eine leichte Schottky-Charakteristik aufweisen. Der Widerstand, der im weiteren Verlauf dieser Arbeit verwendeten $\operatorname{Si}(111)(\sqrt{3} \times \sqrt{3})-\mathrm{Ag}$ Probe, bereinigt um Kontaktwiderstände, beträgt daher höchstens 4,62 k $\Omega$. 


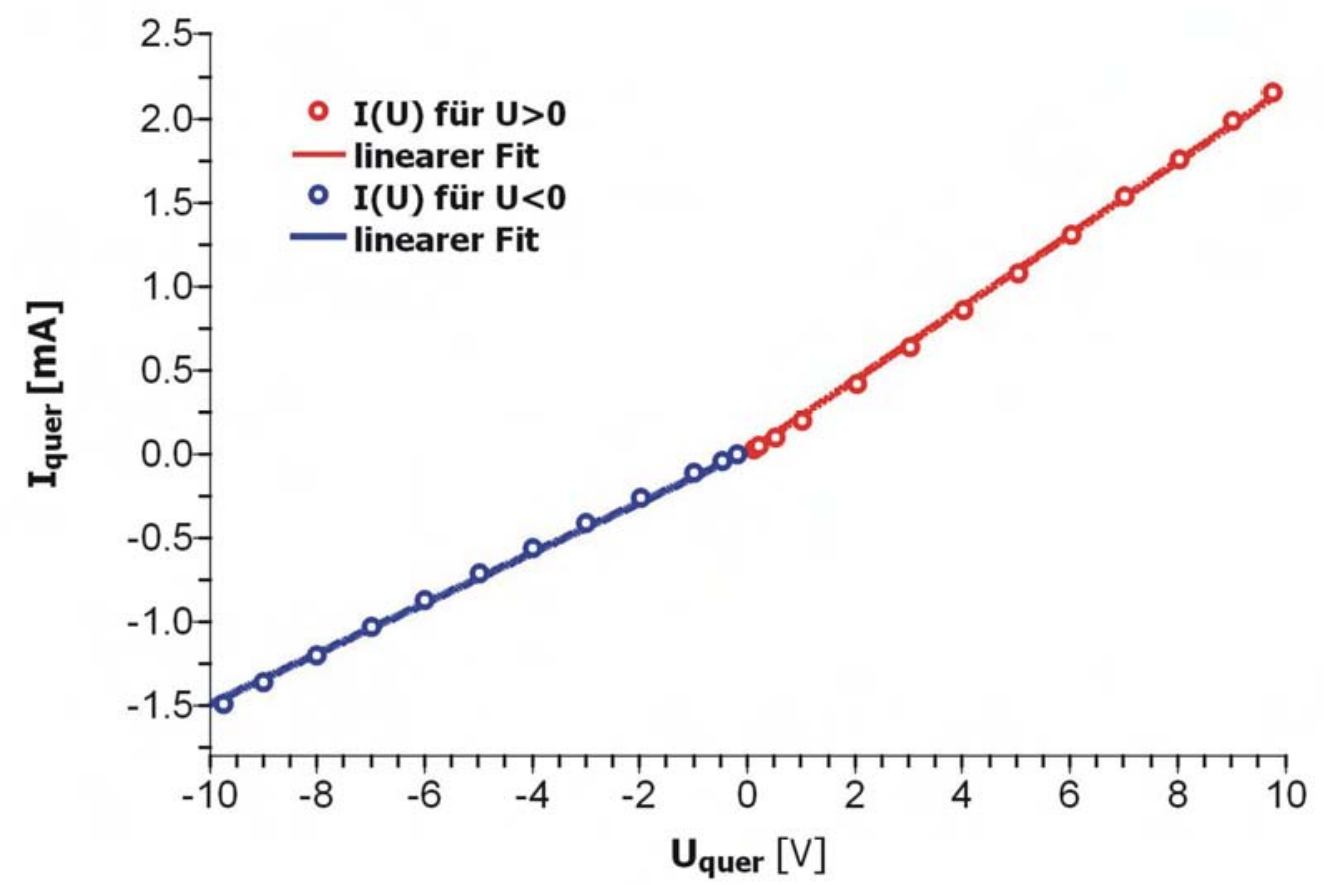

Abbildung 28: I(U)-Kennlinie zur Bestimmung des Gesamtwiderstands der fertig präparierten Si(111)( $\sqrt{3} x \sqrt{3})$-Ag Probe. Die Messung geschah als Zweipol-Messung an den Klemmkontakten. Der Gesamtwiderstands der Probe ist $R_{U>0}=(4,62 \pm 0,14) k \Omega$ und $R_{U<0}=(6,69 \pm 0,20) \mathrm{k} \Omega$.

Mit dem Volumenwiderstand des Si-Wafers von $\sigma_{\mathrm{Si}}=(93 \ldots 130) \Omega \mathrm{cm}$ und den bekannten Abmessungen $(\mathrm{l}=6 \mathrm{~mm}, \mathrm{~b}=2 \mathrm{~mm}, \mathrm{~d}=180 \mu \mathrm{m})$ ergibt sich der Gesamtwiderstand der Probe ohne die $\operatorname{Si}(111)(\sqrt{3} \times \sqrt{3})$-Ag Rekonstruktion zu (16...22) $\mathrm{k} \Omega$. Der Widerstand durch die $\operatorname{Si}(111)(\sqrt{3} \times \sqrt{3})-A g$ Rekonstruktion kann demnach zu (5.8..6.5) $\mathrm{k} \Omega$ abgeschätzt werden, was einer spezifischen Leitfähigkeit von $(460 \ldots 520) \mu \mathrm{S} / \square$ entspricht. ${ }^{19}$ Dies ist eine untere Grenze der Leitfähigkeit durch die $\operatorname{Si}(111)(\sqrt{3} \mathrm{x} \sqrt{3})$-Ag Rekonstruktion, da angenommen wurde, dass keine Kontaktwiderstände von den Kontakten zu Rekonstruktion existieren. Ein Vergleich mit der mikroskopisch bestimmten Leitfähigkeiten durch die $\operatorname{Si}(111)(\sqrt{3} \times \sqrt{3})-\mathrm{Ag}$ Rekonstruktion wird in Kapitel 3.2.2.3 diskutiert.

\footnotetext{
${ }^{19}$ Konkret bedeutet dieser Wert, dass ein Quadrat dieses Materials eine größenunabhängige Leitfähigkeit von $(460 \ldots 520) \mu \mathrm{S}$ besitzt. Für Erklärung der Einheit $(\mu \mathrm{S} / \square)$ wird auf Kapitel 3.1 .2 verwiesen.
} 


\subsubsection{Atomare Struktur}

$\mathrm{Zu}$ Beginn dieser Arbeit wurden zwei unterschiedliche Strukturmodelle der $\operatorname{Si}(111)(\sqrt{3} \times \sqrt{3})-$ Ag Rekonstruktion diskutiert. Die entlang $<11 \overline{2}>$-Richtung symmetrische Oberflächeneinheitszelle, die bei Raumtemperatur bis dato durch STM-Messungen bekannt war, sollte entweder dem HCT-Modell entsprechen oder aber über die zeitliche Mittelung schnell schwankender IET-Domänen zustande kommen. Die Details wurden in Kapitel 2.1.1 ausführlich dargestellt.

Abbildung 29 zeigt eine hochauflösende STM-Topographie der $\operatorname{Si}(111)(\sqrt{3} \mathrm{x} \sqrt{3})-\mathrm{Ag}$ Rekonstruktion bei Raumtemperatur. Die Oberflächeneinheitszelle ist rot eingezeichnet. Sie zeigt keine Spiegelsymmetrie entlang der $<11 \overline{2}>$-Richtung und die beiden Maxima der Einheitszelle entlang $<1 \overline{1} 0>$-Richtung unterschieden sich um etwa $30 \mathrm{pm}$. Nach Ref. [86] wird der helle Kontrast innerhalb der Einheitszelle den kleineren Ag-Trimeren und der dunkle Kontrast den größeren Ag-Trimeren zugeordnet.

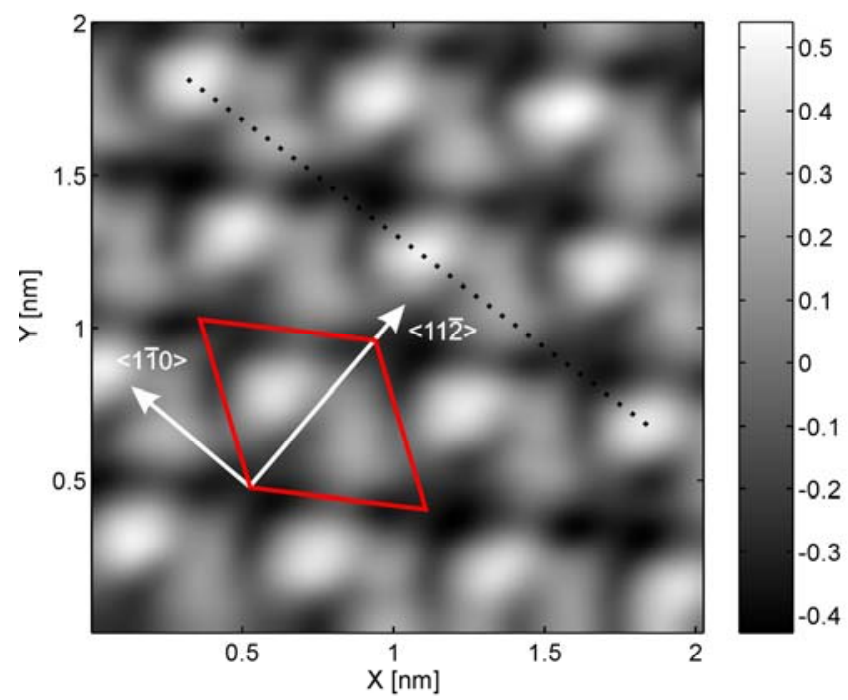

a)

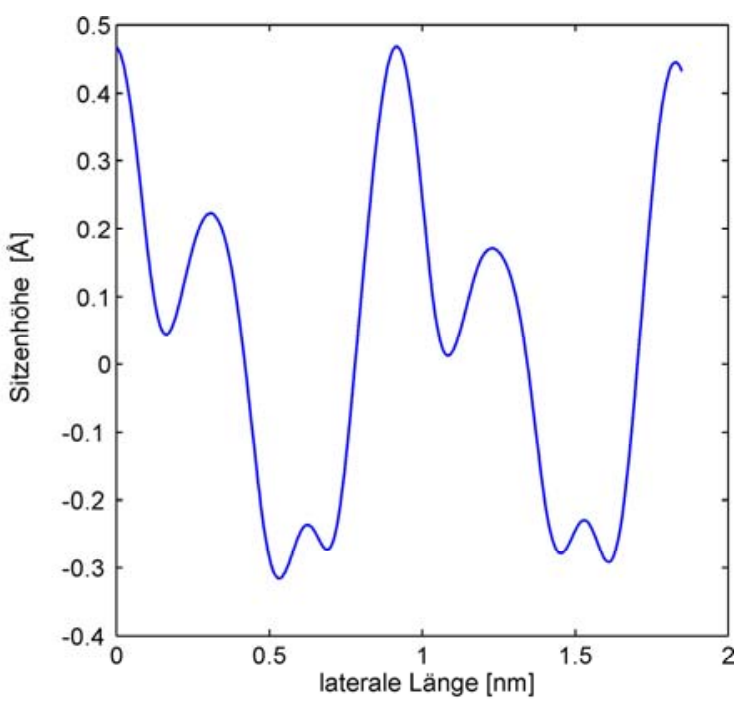

b)

Abbildung 29: (a) $2 \times 2 \mathrm{~nm}^{2}$ STM-Topographie der Si(111) $(\sqrt{3} 3 \mathrm{~V} 3)$-Ag Rekonstruktion bei Raumtemperatur $\left(U_{\text {Bias }}=-0,1 \mathrm{~V} ; I_{T}=4 n A\right)$. Die Einheitszelle der Rekonstruktion ist mit einer Kantenlänge von 6,65 A rot eingezeichnet. Wie für das IET-Modell erwartet, zeigt sie keine Spiegelsymmetrie entlang der $<11 \overline{2}>$-Richtung. (b) zeigt das Höhenprofil entlang der $<1 \overline{1} 0>-$ Richtung gemäß der eingezeichneten Linie in (a). Innerhalb der Einheitszelle beträgt die Höhendifferenz zweier Silbertrimere ungefähr 30 pm. 
Der Kontrast der Asymmetrie wurde energieabhängig, d. h. als Funktion der Bias-Spannung, untersucht. Abbildung 30 zeigt hierzu Topographien im Energieintervall von $\mathrm{U}_{\text {Bias }}=(-0.05 \ldots-0.25) \mathrm{V}$. Bei einer Bias-Spannung von $\mathrm{U}_{\mathrm{Bias}}=-0.05 \mathrm{~V}$ sind alle besetzten Zustände des Oberflächenzustands im Energieintervall von $\mathrm{E}_{\mathrm{f}}$ bis $50 \mathrm{meV}$ darunter am Tunnelprozess beteiligt. Die Korrugation des gezeigten STM-Bilds beträgt bei diesen Abbildungsbedingungen ca. $100 \mathrm{pm}$ und die Asymmetrie entlang der $<1 \overline{1} 0>$-Richtung ist mit ca. $30 \mathrm{pm}$ besonders stark ausgeprägt. Sie ist im gesamten Energieintervall von $\mathrm{U}_{\text {Bias }}=(-0.05 \ldots-0.14) \mathrm{V}$ deutlich zu erkennen. Je mehr gefüllte Zustände des Oberflächenzustands zum STM-Bild beitragen, desto geringer wird jedoch die abgebildete Korrugation. So ist bei $U_{\text {Bias }}=-0.16 \mathrm{~V}$ die Korrugation der Einheitszelle nur noch schwach zu erkennen und verschwindet bei $U_{B i a s}=-0.25 \mathrm{~V}$ vollständig. Eine eindeutige Erklärung dieses Kontrastverlaufs kann im Rahmen der vorliegenden Dissertation nicht abschließend gegeben werden. Eine mögliche Erklärung könnte sein, dass die Überschussenergie der im $\mathrm{S}_{1}$-Zustand verbliebenen Löcher zur Anregung der IET-Struktur ausreichend ist, da nach DFTBerechnungen die Gesamtenergie der IET-Struktur nur $0.1 \mathrm{eV}$ pro Einheitszelle tiefer liegt als die der HCT-Struktur [86, 100].

Diese STM-Daten bei Raumtemperatur entsprechen damit STM-Untersuchungen bei tiefen Temperaturen und zeigen eindrucksvoll, das auch bei Raumtemperatur eine asymmetrische Einheitszelle entsprechend dem IET-Modell vorliegt, was zu Beginn dieser Arbeit noch kontrovers diskutiert worden ist. ${ }^{20}$

${ }^{20}$ Diese Daten wurden im November 2006 gemessen und ausgewertet. Zeitgleich mit den eigenen Ergebnissen publizierten Zhang et. al. STM-Messungen die ebenfalls bestätigen, dass das HCT-Modell bei Raumtemperatur nicht zutrifft [65]. Eine bereits begonnene Veröffentlichung der eigenen Ergebnisse musste daraufhin verworfen werden. 

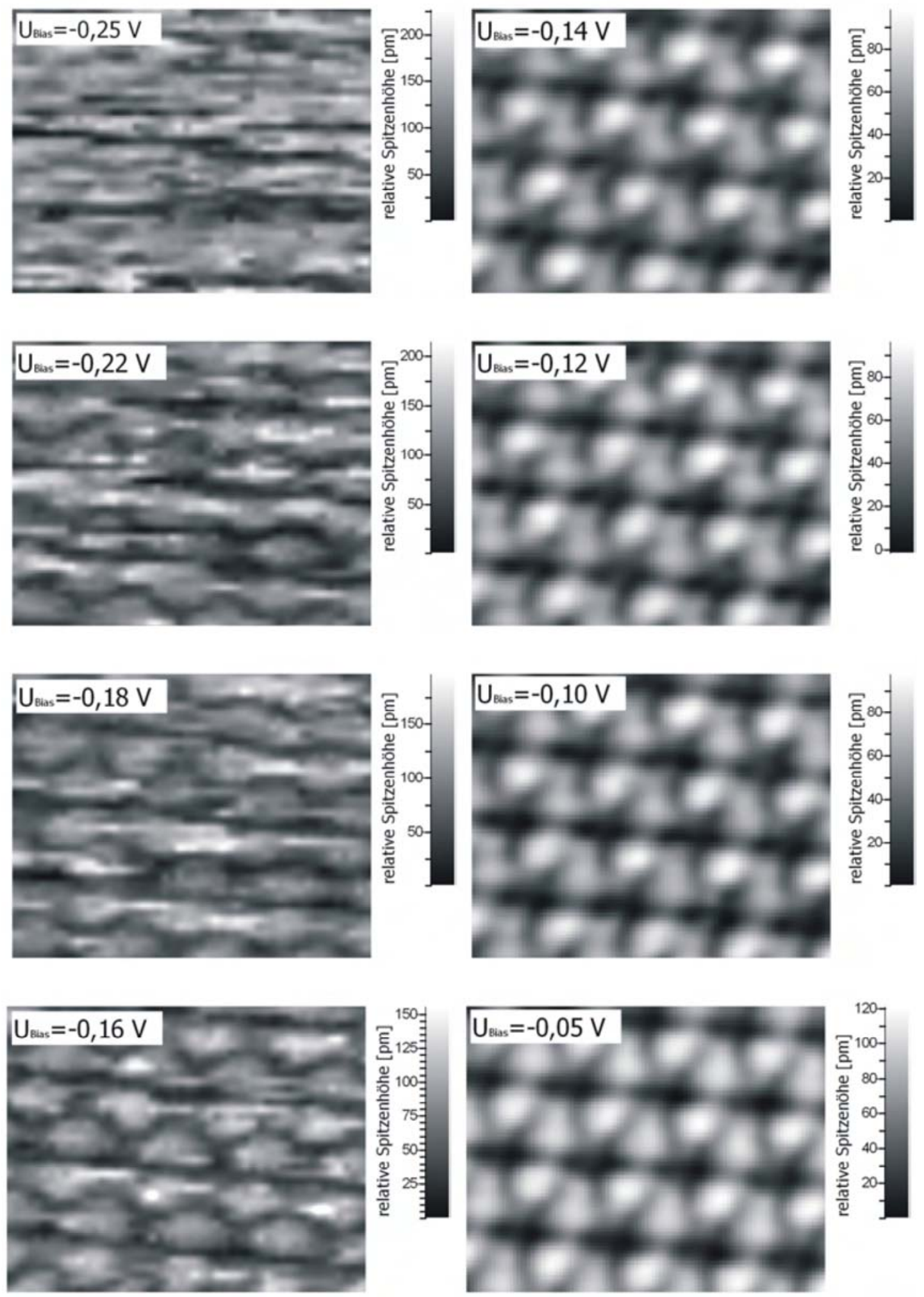

Abbildung 30: Multi-Bias-Topographien $\left(2 \times 3 \mathrm{~nm}^{2}\right)$ bei $I_{t}=4 \mathrm{nA}$ und den jeweils angegeben Spannungen. Deutlich zu erkennen ist das Einsetzen der atomaren Korrugation mit dem Einsetzen des $S_{l}$-Oberflächenzustands. Abbildung aus Ref.[36]. 


\subsubsection{Silber-Atome als Donatoren auf der $\mathrm{Si}(111)(\sqrt{ } 3 \times \sqrt{ } 3)-A g$ Rekons- truktion}

Ag-Adatome wirken als Donatoren und diffundieren als positive Ionen auf der Oberfläche. Aufgrund ihrer hohen Mobilität können sie bei Raumtemperatur bei üblichen Samplingraten mit dem STM nicht abgebildet werden. Da es sich um einen thermisch aktivierten Prozess handelt, ist bei tiefen Temperaturen die Mobilität derart verringert, dass ein direktes Abbilden möglich ist [93]. Dies ist bei 5,6 K gelungen und Abbildung 31 zeigt entsprechende STMTopographien. Einzelne Ag-Adatome lassen sich durch ihre charakteristische, sternartige Erhebung mit drei erhöhten Ag-Trimeren zwischen den „Flügeln“ anhand von publizierten STM-Messungen leicht identifizieren [93]. Hierbei entspricht jeder „Stern“ einem AgAdatom. Sowohl Adatome als auch ihre Vakanzen in der geschlossenen Rekonstruktion werden beobachtet.
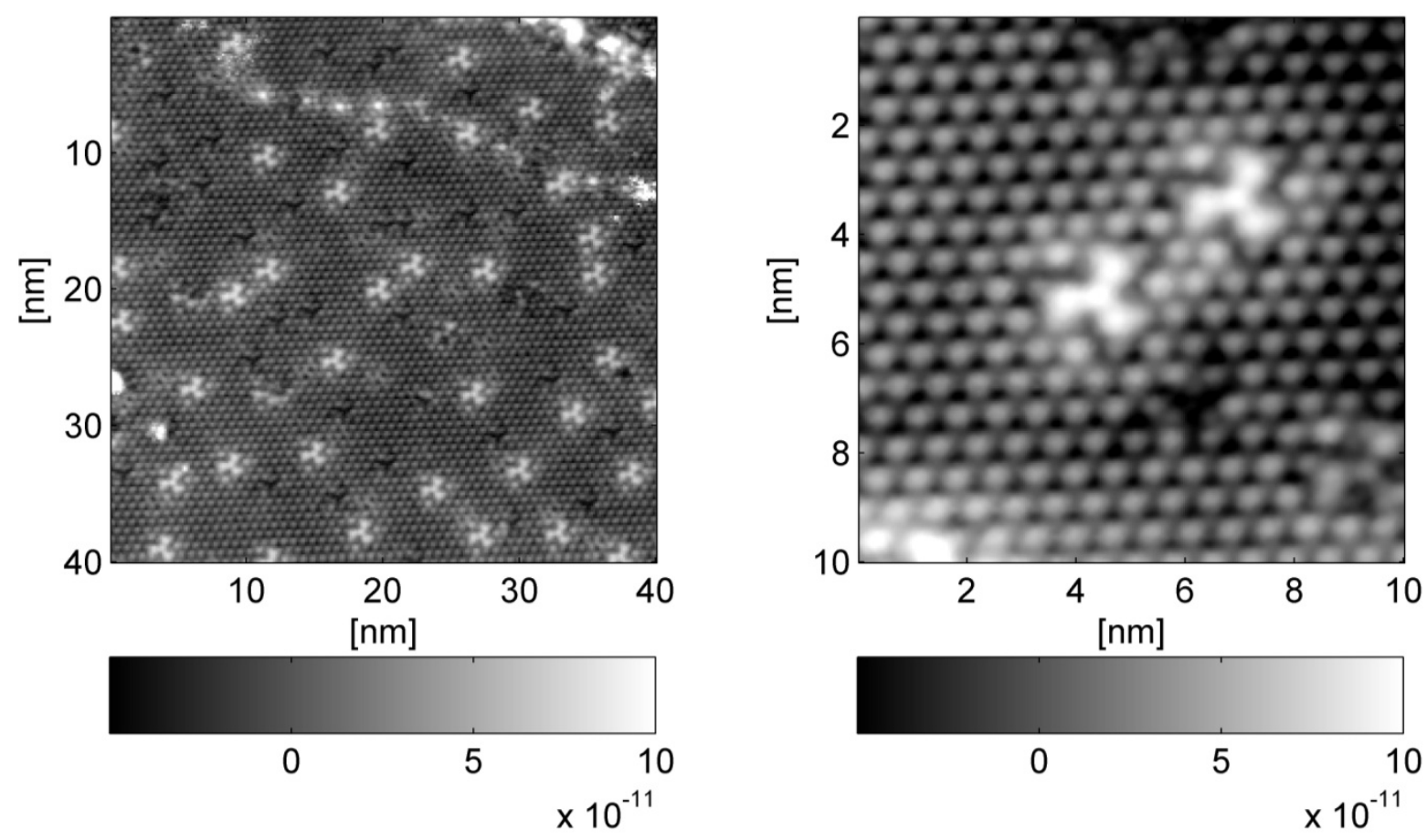

Abbildung 31: STM-Topographien bei $5.6 \mathrm{~K}$ zeigen das ausgefrorene Ag-Adatomgas. (a) Sowohl Adatome als auch Vakanzen werden gefunden $\left(U_{\text {Bias }}=1 V ; I_{t}=0,5 n A\right)$. (b) Hochauflösende Topographie zweier Adatome $\left(U_{\text {Bias }}=0,5 \mathrm{~V} ; I_{t}=0,5 \mathrm{nA}\right)$.

Die Adatome sind umgeben von einem erhöhten Saum, welcher als lokale Oberflächenbandverbiegung verstanden wird. Sie resultiert aus der positiven Ladung des Adatoms, welches durch die delokalisierte Ladungsdichte des 2DEG abgeschirmt wird. Eine Analyse der 
räumlichen Ladungsdichte und des elektrostatischen Potentials um ein Ag-Adatom herum ist in Ref. [99] zu finden. Aufgrund des Fehlens einer Oberflächenbandverbiegung wird vermutet, dass die Vakanzen im Gegensatz zu den Adatomen nicht geladen sind.

Auf großskaligen Topographien erkennt man deutlich, dass die Abstände zwischen einzelnen Adatomen nicht zufallsverteilt sind. Es scheint einen Mindestabstand zu geben und eine Vielzahl von Adatomen besitzt einen größtmöglichen Abstand zum nächsten Nachbarn. Dieses Verhalten ist im Einklang mit einer repulsiven Coulomb-Wechselwirkung zwischen positiven Ionen. Ein entsprechendes Ordnungsphänomen wird auch auf Oberflächen von III-V Halbleitern in der Nähe geladener Oberflächenstufen beobachtet [101]. 


\subsubsection{Perkolationsbildung durch Selbstorganisation}

Abbildung 32 zeigt $1.5 \times 1.5 \mu \mathrm{m}^{2}$ der $\operatorname{Si}(111)(\sqrt{3} \times \sqrt{3})$-Ag Rekonstruktion der verwendeten

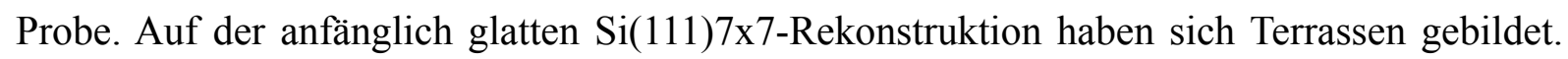
Die Terrassen sind ineinander verwoben und haben keine eindeutige Größenverteilung. Es lassen sich Bereiche identifizieren, in denen große, miteinander verbundende Terrassen dominieren. Hier befinden sich atomar glatte Bereiche von $100 \mathrm{~nm}$ Durchmesser. In anderen Probenbereichen ist die mittlere Strukturgröße wesentlich kleiner. Zusammenhängende Bereiche sind jedoch auch hier zu finden. Der Höhenunterschied zwischen den Terrassen beträgt $3.06 \AA$, was im Rahmen der Piezo-Eichung einer monoatomaren Si(111)-Ebene von $3.14 \AA$ entspricht.
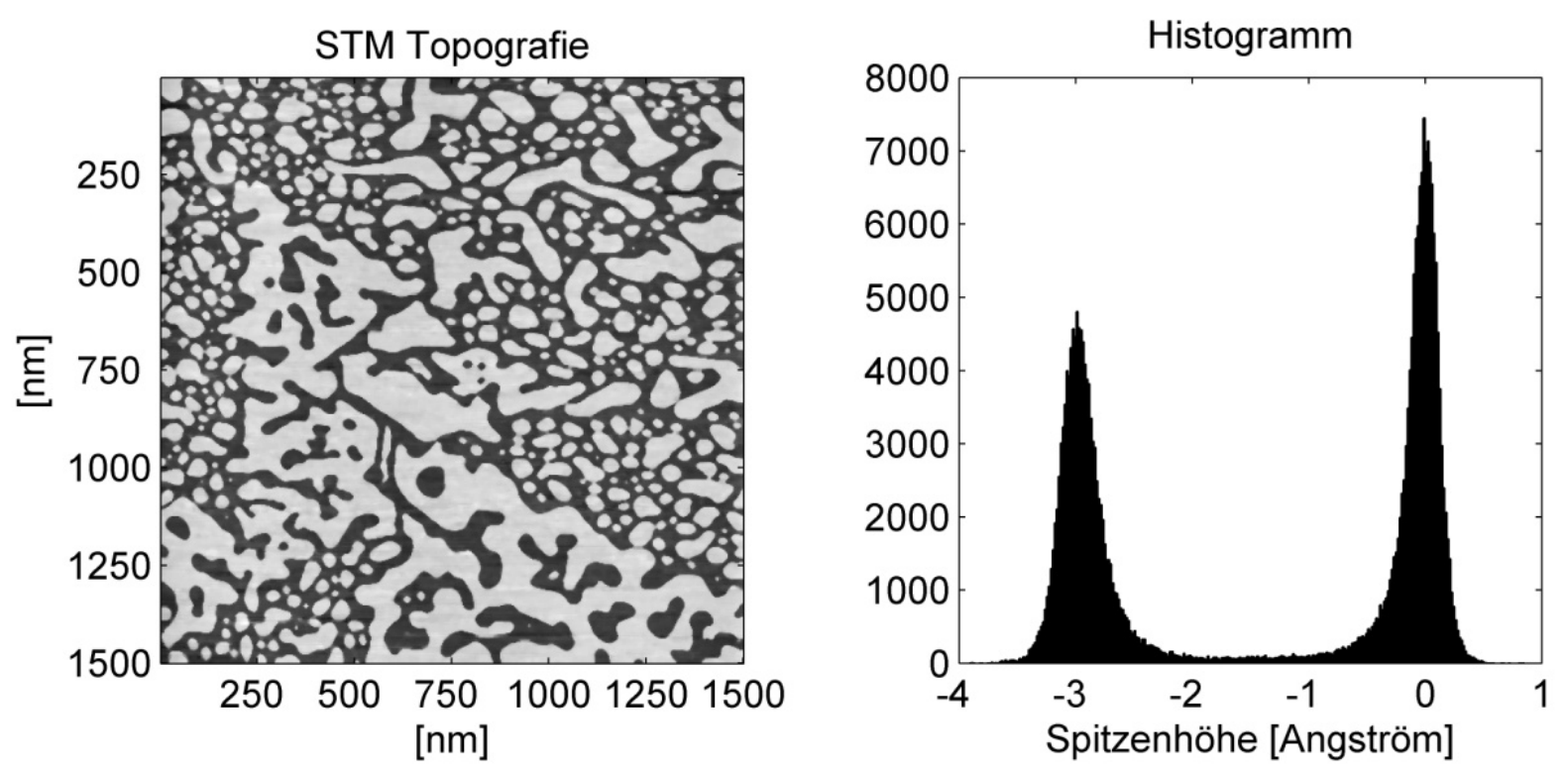

Abbildung 32: (a) Auf einer anfänglich atomar glatten Si(111)-Oberfläche entsteht beim Bilden der Si(111)( $\sqrt{3} 3 x \sqrt{ } 3)-A g$ Rekonstruktion eine komplizierte Perkolationsstruktur bestehend aus zwei Terrassen. Beide Terrassen sind durch eine Monolage Si(111) d. $h$. $d_{S i(111)}=3,14$ A voneinander getrennt. (b) zeigt das entsprechende Histogramm. 


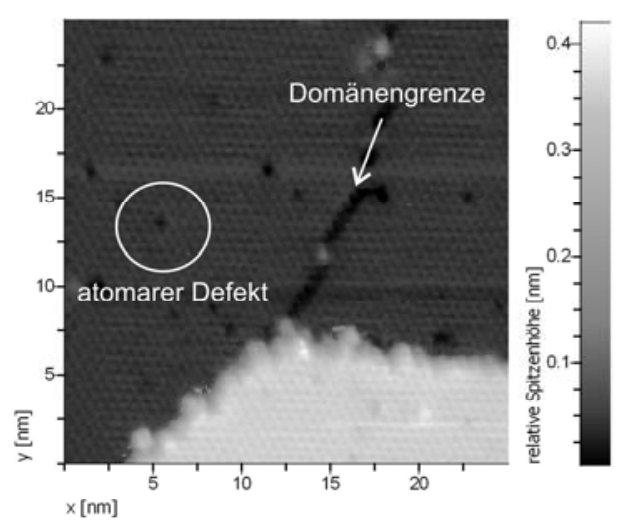

a)

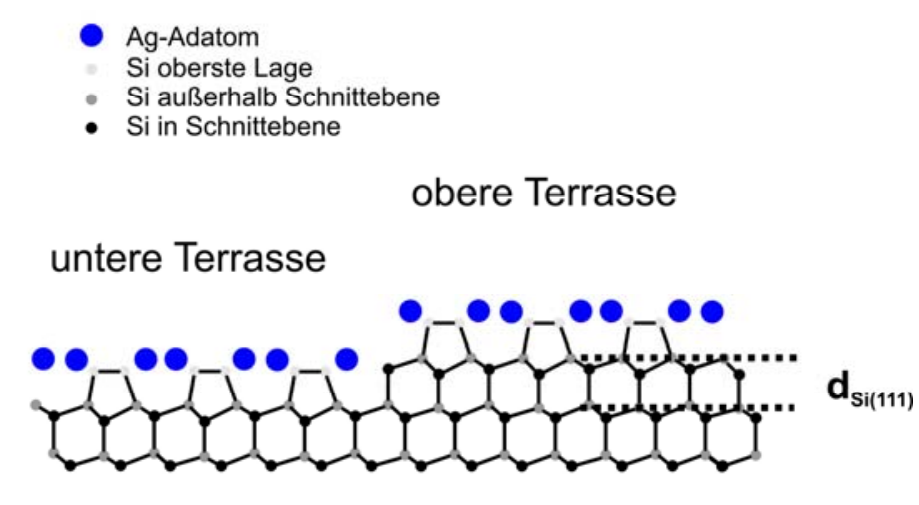

b)

Abbildung 33: a) Hochauflösende Topographie einer Terrassenstufe bei Raumtemperatur. Deutlich ist die Korrugation der Si(111) $(\sqrt{ } 3 x \sqrt{ } 3)$-Ag Rekonstruktion auf beiden Terrassen zu erkennen. Einzelne Defekte und eine Domänengrenze sind ebenso ersichtlich. b) zeigt eine schematische Seitenansicht in der (110)-Ebene der atomaren Struktur einer Terrassenstufe [73, 102]. Die Terrassen unterscheiden sich lediglich durch eine Si(111)-Ebene und besitzen dieselbe atomare Struktur. Abbildung angelehnt an Ref. [36, 103].

Abbildung 33 (a) zeigt eine atomar auflösende Topographie einer Terrassenstufe bei Raumtemperatur. Die Korrugation der Si(111) $(\sqrt{ } 3 \mathrm{x} \sqrt{ } 3)$-Ag Rekonstruktion bedeckt sowohl die untere als auch die obere Terrasse. Atomare Defekte und eine Domänengrenze sind ersichtlich. (b) illustriert schematisch die atomare Struktur einer Terrassenstufe. Die Terrassenhöhen unterscheiden sich durch eine Si(111)-Ebene, entsprechend $\mathrm{d}_{\mathrm{Si}(111)}=3,14 \AA$. Ansonsten sind ihre Oberflächen identisch [103]. In Analogie zur Struktur wurden die elektronischen Eigenschaften mit Raster-Tunnel-Spektroskopien auf beiden Terrassen analysiert. Unterschiede wurden nicht gefunden.

Die Ursache für die Terrassenbildung auf einem anfänglich atomar glattem Si(111)-Substrat liegt an einem Überangebot an Silizium bei der Formation der $\operatorname{Si}(111)(\sqrt{3} \times \sqrt{3})-A g$ Rekonstruktion [104]. Die Dichte von Si-Atomen auf der anfänglichen Si(111)-7x7 Oberfläche beträgt $2.08 \times\left(7.8 \quad 10^{14}\right) \mathrm{Si} / \mathrm{cm}^{2}$. Die Si-Dichte in der $\operatorname{Si}(111)(\sqrt{3} \times \sqrt{3})-\mathrm{Ag}$ Rekonstruktion beträgt jedoch nur die Hälfte [104]. Während des Übergangs der einen in die andere Oberflächenrekonstruktion ist folglich überschüssiges Silizium vorhanden. Die auf der Si(111)-7×7 Oberfläche adsorbierten Silberatome binden an Si-Trimere der obersten Si-Lage und erzeugen dabei jeweils ein freies Si-Atom. Die überschüssigen Si-Atome diffundieren zu angrenzenden $\mathrm{Si}(111)-7 \times 7-G e b i e t e n$, reagieren dort mit diffundierenden Ag-Atomen und bilden eine um eine $\operatorname{Si}(111)$-Lage höhere $\operatorname{Si}(111)(\sqrt{3} 3 \mathrm{~V} \sqrt{3})$-Ag Terrasse (siehe 
Abbildung 33 (b)). Wird, wie bei der hier verwendeten Probe, genügend Silber während der Deposition angeboten, nimmt die relative Bedeckung der unteren und oberen $\operatorname{Si}(111)(\sqrt{3} x \sqrt{ } 3)$ Ag Domäne auf Kosten der Si(111)-7×7-rekonstruierten Oberfläche zu, bis die gesamte Oberfläche in ein Netzwerk von zwei $\operatorname{Si}(111)(\sqrt{3} \mathrm{x} \sqrt{3})$-Ag rekonstruierten Terrassen übergeht $[103,104]$.

Die räumliche Struktur dieses Netzwerks sollte von den mikroskopischen Prozessen während der Nukleation und Diffusion abhängig sein. So könnte bspw. die Temperatur, als Parameter der mittleren freien Diffusionslänge, einen Einfluss auf die Strukturbildung haben. Ebenso ist denkbar, dass Defekte der 7x7-Rekonstruktion die Nukleation beeinflussen. Experimentell wurde beobachtet, dass die Terrassenbildung in der Nähe von 7x7-Substratstufen unterbunden wird. Das überschüssige Silizium scheint sich in diesen Bereichen an die Substratstufe anzulagern und nicht zu einer Terrasse zu nukleiren (siehe hierzu Abschnitt 3.3). 


\subsection{Zusammenfassung}

Die atomare und elektronische Struktur der $\operatorname{Si}(111)(\sqrt{3} \mathrm{x} \sqrt{3})-A g$ Rekonstruktion wurde detailliert untersucht. Die Ergebnisse zeigen, dass nicht nur bei tiefen Temperaturen die asymmetrische IET-Struktur vorliegt, sondern auch bei Raumtemperatur.

Die $\operatorname{Si}(111)(\sqrt{3} \mathrm{x} \sqrt{3})-\mathrm{Ag}$ Rekonstruktion besitzt einen parabolischen, isotropen und zweidimensionalen Oberflächenzustand, der durch zusätzliche überschüssige Ag-Adatome doniert werden kann. Elektronen propagieren in diesem Zustand wie freie, ebene Wellen, so dass sie sich wie ein zweidimensionales freies Elektronengas (2DEG) verhalten. Die Besetzung des $\mathrm{S}_{1}$-Zustands der verwendeten Probe wurde durch STS zu $\mathrm{E}_{\mathrm{f}}=250 \mathrm{meV}$ spezifiziert. Mit der sich aus Literaturwerten ergebenden effektiven Masse von $\mathrm{m}^{*}=0.13$ beträgt die Ladungsträgerdichte $n_{2 D E G}=\frac{2 m^{*} E}{2 \pi \hbar^{2}}$ damit $1.610^{13} \mathrm{e}^{-} / \mathrm{cm}^{2}$ und die Fermiwellenlänge kann zu $\lambda_{\mathrm{F}}=68 \AA$ bestimmt werden [92].

Ein elektrisch leitfähiges 2DEG konnte somit direkt auf der Oberfläche erzeugt und in situ im UHV kontaktiert werden. Die untere Grenze für die Leitfähigkeit durch die $\operatorname{Si}(111)(\sqrt{3} x \sqrt{ } 3)$ Ag Rekonstruktion wird zu (460...520) $\mu \mathrm{S} / \square$ quantifiziert.

Die Oberfläche der verwendeten Probe besteht aus einem Netzwerk aus Terrassen, die sich um eine Si(111)-Ebene (3,14 Å) unterscheiden. Die Eigenschaften der unteren und oberen Terrasse sind hinsichtlich ihrer atomaren und elektronischen Eigenschaften identisch. 


\section{Ladungstransport durch die $\operatorname{Si}(111)(\sqrt{3} \times \sqrt{3})-A g$ Rekonstruktion}

Bevor die mikroskopischen Messungen zum Ladungstransport durch die $\operatorname{Si}(111)(\sqrt{3} x \sqrt{3})-A g$ Rekonstruktion mittels Raster-Tunnel-Potentiometrie vorgestellt werden, wird zunächst ein Überblick über den Stand der Forschung gegeben.

\subsection{Stand der Forschung}

\subsubsection{Die elektrische Leitfähigkeit der $\operatorname{Si}(111)(\sqrt{ } 3 \times \sqrt{ } 3)-A g$ Rekonstruktion}

Makroskopische Ladungstransportmessungen auf der $\operatorname{Si}(111)(\sqrt{3} \mathrm{x} \sqrt{3})-\mathrm{Ag}$ Rekonstruktion begannen Anfang der 90er Jahre [76]. In Vierpunktgeometrie mit Klemmen oder variablen Spitzen, ähnlich dem eines STMs, wurde versucht, die Leitfähigkeit durch die Oberflächenrekonstruktion zu bestimmen und von der Volumenleitfähigkeit des Silizium-Substrats zu diskriminieren. Einhergehend mit der technologischen Weiterentwicklung konnte der Elektrodenabstand von anfänglichen Millimetern bis auf aktuell wenige Mikrometer verringert werden.

Tabelle 1 zeigt eine Zusammenfassung publizierter Literaturwerte, welche maßgeblich in der Arbeitsgruppe von Shuji Hasegawa entstanden sind. Eine große Variation der Leitfähigkeiten durch die Rekonstruktion ( $\left.\sigma_{\text {Oberfläche }}\right)$ von $(20 \ldots 3000) \mu \mathrm{S} / \square$ ist zu beobachten. Tendenziell werden größere Leitfähigkeiten bei kleineren Elektrodenabständen gefunden. Eine Interpretation dieses Sachverhalts wird in Kapitel 3.1.2 gegeben. Tabelle 1 zeigt zudem die errechneten Leitfähigkeiten durch den Bereich der Oberflächenbandverbiegung im Silizium. Bis auf eine Referenz spezifizieren die übrigen Autoren die Leitfähigkeit durch den Bereich der Raumladungszone auf etwa $50 \mu \mathrm{S} / \square$. 


\begin{tabular}{|l|l|l|l|l|}
\hline Referenz & Elektrodenabstand & Substrat & $\boldsymbol{\sigma}_{\text {Oberfläche }}$ & $\boldsymbol{\sigma}_{\text {Verarmungszone }}$ \\
\hline$[105]$ & $8 \ldots 10 \mu \mathrm{m}$ & n-dotiert & $20 \mu \mathrm{S} / \square$ & $20 \mu \mathrm{S} / \square$ \\
\hline$[63]$ & $5.6 \mathrm{~mm}$ & n-dotiert $(11-100) \Omega \mathrm{cm}$ & $(38 \pm 8) \mu \mathrm{S} / \square$ & $(111 \pm 7) \mu \mathrm{S} / \square$ \\
\hline [78] & mehrere Millimeter & p-dotiert $20 \Omega \mathrm{cm}$ & $(115 \pm 5) \mu \mathrm{S} / \square$ & $40 \mu \mathrm{S} / \square$ \\
\hline$[106]$ & $8 \mu \mathrm{m}$ & n-dotiert $(10-100) \Omega \mathrm{cm}$ & $600 \mu \mathrm{S} / \square$ & $50 \mu \mathrm{S} / \square$ \\
\hline$[107]$ & $100 \mu \mathrm{m}$ & n-dotiert $(5-15) \Omega \mathrm{cm}$ & $700 \mu \mathrm{S} / \square$ & $50 \mu \mathrm{S} / \square$ \\
\hline$[106]$ & $20 \mu \mathrm{m}$, Si stufenfrei & n-dotiert $(10-100) \Omega \mathrm{cm}$ & $800 \mu \mathrm{S} / \square$ & $50 \mu \mathrm{S} / \square$ \\
\hline$[107]$ & $10 \mu \mathrm{m}$ & n-dotiert $(5-15) \Omega \mathrm{cm}$ & $1000 \mu \mathrm{S} / \square$ & $50 \mu \mathrm{S} / \square$ \\
\hline$[107]$ & $1 \mu \mathrm{m}$ & n-dotiert $(5-15) \Omega \mathrm{cm}$ & $1500 \mu \mathrm{S} / \square$ & $50 \mu \mathrm{S} / \square$ \\
\hline$[108]$ & $10 \mu \mathrm{m}$ & p-dotiert $190 \Omega \mathrm{cm}$ & $3000 \mu \mathrm{S} / \square$ & - \\
\hline
\end{tabular}

Tabelle 1: Zusammenfassung publizierter Literaturwerte zur Leitfähigkeit der

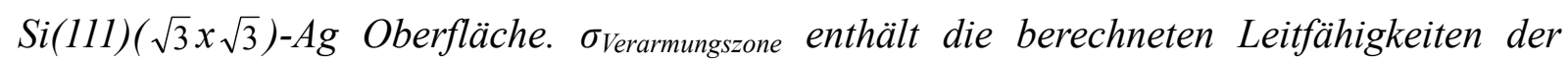
Verarmungszone unter der Oberfläche. Referenz [106] hat durch spezielle Probenpräparation eine stufenfreie Si(111)7x7-Oberfläche generiert.

Für n-dotiertes Silizium (100 $\Omega \mathrm{cm})$, wie es in dieser Arbeit verwendet worden ist, zeigt Abbildung 34 die Leitfähigkeit durch den Bereich der Raumladungszone als Funktion der Oberflächenvalenzbandkante und damit letztendlich als Funktion der Besetzung des $\mathrm{S}_{1}$ Zustands (siehe Abschnitt 2.1.2) im Detail. Die energetische Position der Oberflächenvalenzbandkante wurde für die im weiteren Verlauf dieser Arbeit verwendeten Proben gemäß Abbildung 22 (a) für eine Besetzung des Oberflächenzustands von $250 \mathrm{meV}$ abgeschätzt. Sie ist als grüner Bereich in Abbildung 34 eingezeichnet. Die zu erwartende Leitfähigkeit durch die Raumladungszone, welche als Kurzschlusskanal für den Ladungstransport durch den $\mathrm{S}_{1}$-Zustand fungieren würde, wird hiernach ebenfalls zu maximal $\sim 50 \mu \mathrm{S} / \square$ abgeschätzt. 

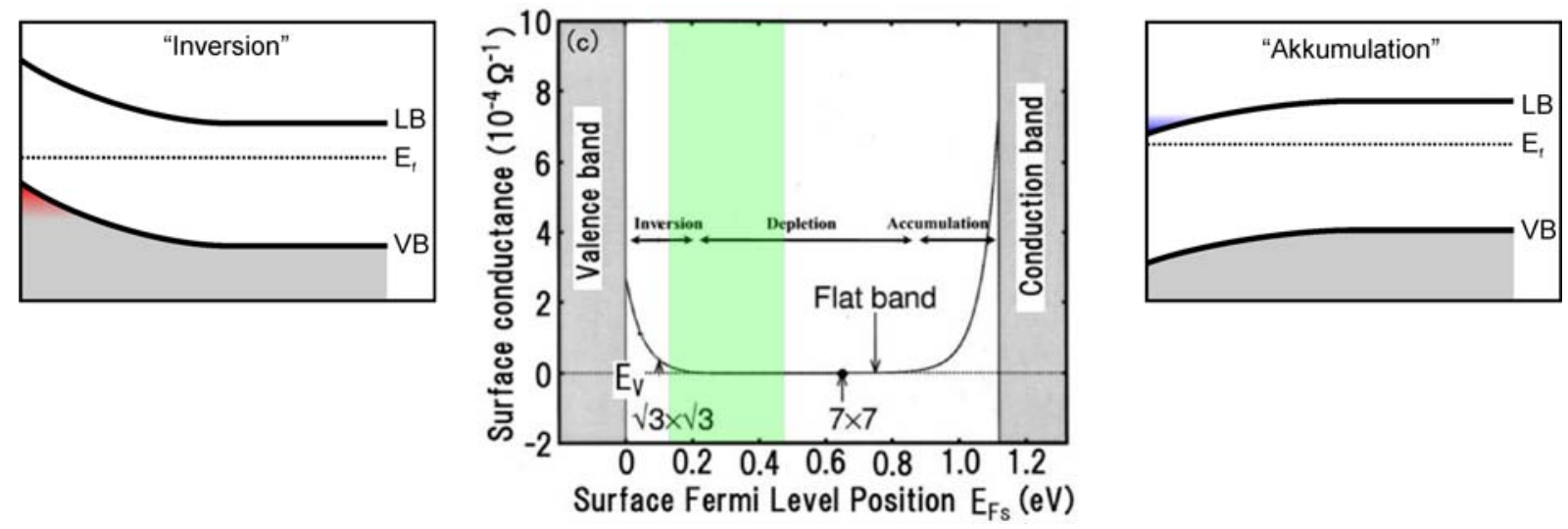

Abbildung 34: Berechnete Leitfähigkeit durch die Raumladungszone als Funktion der Oberflächenvalenzbandkante für n-dotiertes Silizium ( 100 $\Omega \mathrm{cm})$. Hierfür wurden die freien Ladungsträgerdichten im Valenz- und Leitungsband als Funktion der Oberflächenbandverbiegung bei Raumtemperatur berechnet und auf Grundlage der jeweiligen Beweglichkeiten ( $\mu$ ) für Löcher und Elektronen in eine Leitfähigkeit umgerechnet. Der grüne Bereich markiert die zu erwartenden Leitfähigkeiten durch die Raumladungszone gemäß experimenteller Resultate zur Oberflächenbandverbiegung für die im Rahmen der vorliegenden Arbeit verwendeten Proben (siehe hierzu Abbildung 22 (a) bzw. Ref. [90]). Piktogramme der Bandverbiegung illustrieren die von den Autoren verwendeten Begriffe (Inversion und Akkumulation) bei den jeweiligen Situationen. Abbildung gemäß Ref. [106] mit leichten Veränderungen.

Im Kapitel 2.1.2 wurde bereits erörtert, dass sich die Ladungsträgerdichte im $\mathrm{S}_{1}$-Zustand durch Ag-Adatome gezielt verändern lässt. Dies sollte sich auch in einer Veränderung von

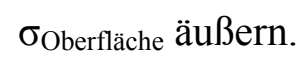

Abbildung 35 zeigt experimentelle Ergebnisse zur Veränderung der Leitfähigkeit während der Adsorption von Silber auf der $\operatorname{Si}(111)(\sqrt{3} \times \sqrt{3})$-Ag Oberfläche nach Ref [109]. Wie aufgrund der bereits vorgestellten Eigenschaften zu erwarten war, sinkt bei jeder Deposition der Widerstand. Jedoch lassen sich zwei unterschiedliche Bereiche identifizieren. Während der ersten drei Depositionen (A-C) verbleibt der Widerstand auch nach Ende der Deposition beim jeweils kleinsten Widerstand. Nachdem 0.032 ML deponiert worden sind, verändert sich dieser Verlauf und der Widerstand steigt nach erfolgter Deposition an (D-F). 


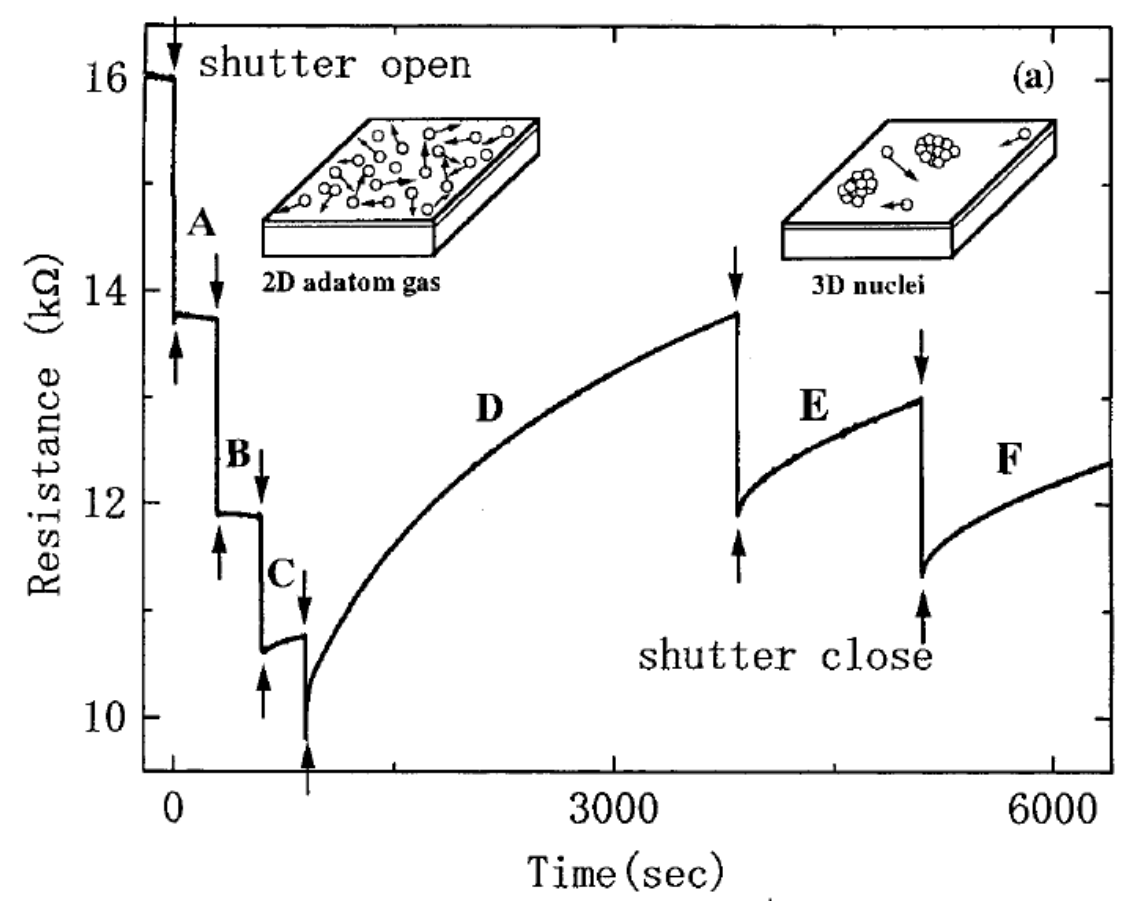

Abbildung 35: Veränderung der Oberflächenleitfähigkeit während der Adsorption von Silber auf der Si(111) $(\sqrt{3} x \sqrt{3})$-Ag Oberfläche bei Raumtemperatur, gemessen in Vierpunktgeometrie. Die Deposition wurde mit einer Rate von 0.24 ML/Min auf n-dotiertem Silizium (100 $\Omega \mathrm{cm})$ ausgeführt. Abwärtszeigende Pfeile kennzeichnen den Beginn einer Deposition und aufwärtszeigende das Ende. Bei jeder Deposition (Dauer zwei Sekunden) wurden 0.008 ML Silber adsorbiert. Bis zu einer Adatomdichte von 0.032 ML sinkt der Widerstand bei jeder Deposition. Darüber nukleiren die Adatome zu Ag-Kristallen. Abbildung aus Ref. [109].]

Der zugrunde liegende physikalische Prozess für dieses Verhalten ist schematisch in Abbildung 35 skizziert. Bis zu einer kritischen Bedeckung von 0.032 ML diffundieren die Adatome als Monomere auf der Oberfläche. Durch die eingeschränkte Mobilität beschreiben viele Autoren diesen Zustand als ein zweidimensionales Adatomgas [93, 110]. In diesem Zustand sind die Adatome ionisiert und donieren den Oberflächenzustand. Oberhalb der kritischen Bedeckung koaleszieren die Ag-Adatome jedoch und bilden Kristallite auf der Oberfläche [110]. Im koaleszierten Zustand stehen die Adatome nicht mehr als Donatoren zur Verfügung, was mit einem Anstieg des Widerstands einhergeht. Die zeitliche Dynamik des Nukleationsprozesses lässt sich durch den langsam ansteigenden Widerstand nach erfolgter Deposition beobachten (siehe D) [110]. 


\subsubsection{Kenntnisstand zu den mikroskopischen Transporteigenschaften}

Ab 2002 waren Vierpunktmessungen technologisch derart weit entwickelt, dass die Oberflächenleitfähigkeit auf der $\operatorname{Si}(111)(\sqrt{3} \times \sqrt{3})-\mathrm{Ag}$ Rekonstruktion mit räumlichen Auflösungen im Mikrometerbereich untersucht werden konnte [107]. Dadurch gelang es, mikroskopische Prozesse des makroskopischen, elektrischen Widerstands in diesem System zu studieren. In Abbildung 36 sind hierzu interessante Ergebnisse aus Vierpunktmessungen dargestellt. Die gemessene Leitfähigkeit variiert mit dem Elektrodenabstand und bei kleineren Abständen werden höhere Leitfähigkeiten bestimmt. Die Autoren erklären diesen Trend mit der Anwesenheit extrinsischer Defekte in der Oberfläche, die als zusätzliche Streuzentren dienen [107]. Bei verringertem Elektrodenabstand ist die Wahrscheinlichkeit durch ein Streuzentrum wie z. B. eine Substratstufe beeinflusst zu werden geringer, was den Anstieg in der Leitfähigkeit erklärt.

Abbildung 36 (b) zeigt eine systematische Vierpunktmessung mit $8 \mu \mathrm{m}$ Elektrodenabstand zum Einfluss von Substratstufen auf die Leitfähigkeit der Oberfläche. Durch geeignete Temperaturführung während der Probenpräparation wurden Stufen auf der Oberfläche des Siliziums zu „Stepbunching“-Bereichen hoher Stufendichte koalesziert [111-113]. Die „Stepbunching“-Bereiche sind getrennt durch mikrometergroße, atomar glatte $\mathrm{Si}(111)-7 \mathrm{x} 7$ Terrassen. Die Autoren setzen voraus, dass die Präparation der $\operatorname{Si}(111)(\sqrt{3} \times \sqrt{3})-\mathrm{Ag}$ Rekonstruktion die strukturellen Eigenschaften des Si-Substrats nicht verändert. Der gemessene Widerstand über „Stepbunching“-Bereiche hinweg ist bis zu fünfmal höher als auf den „Stepbunching“-freien Terrassen. Substratstufen haben aufgrund dieser Ergebnisse einen wichtigen Anteil an der makroskopisch gemessenen Leitfähigkeit durch die Rekonstruktion [107]. Spezifiziert wurde die Leitfähigkeit über Substratstufen erstmalig Ende 2004 von Matsuda et. al. [114, 115]:

I. Auf vizinalen $\mathrm{Si}(111)$-Substraten mit einem Stufenabstand von (10...20) nm wurde die Leitfähigkeit der Oberfläche als Funktion des Winkels zu den Substratstufen vermessen. Aus der Anisotropie der Leitfähigkeit und mit Kenntnis der Stufendichte wurde die Leitfähigkeit über Substratstufen zu $\sigma_{\text {Stufe }}=3000 \Omega^{-1} \mathrm{~m}^{-1}$ bestimmt. 


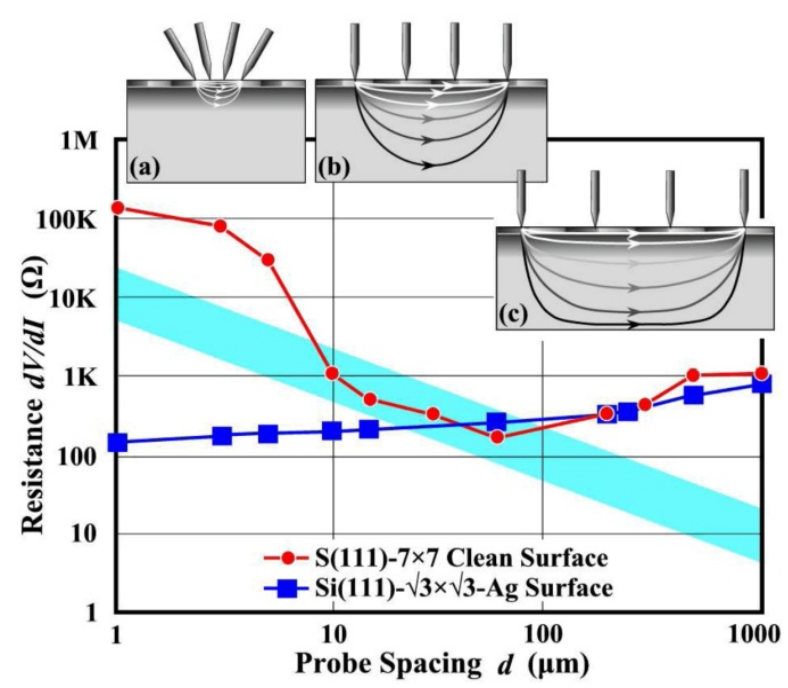

(a)

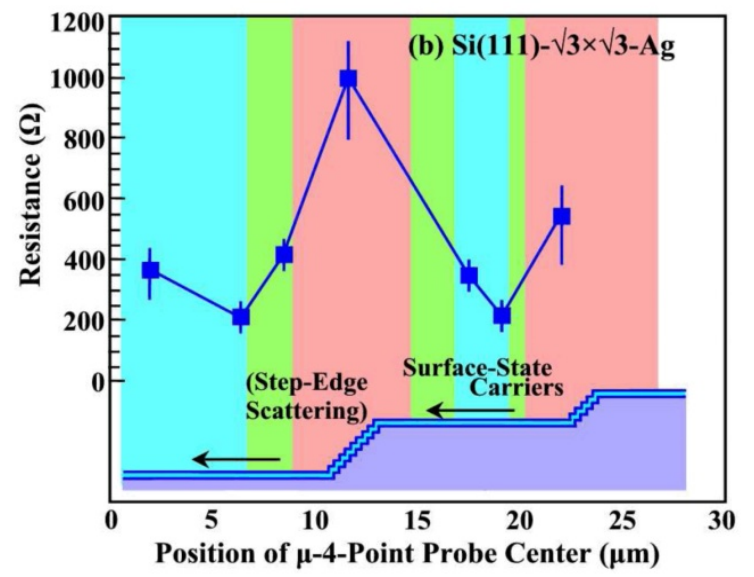

(b)

Abbildung 36: (a) Widerstand der Si(111) $(\sqrt{3} x \sqrt{3})$-Ag Rekonstruktion bei Raumtemperatur gemessen in Vierpunktgeometrie mit variablem Elektrodenabstand (blau). Die Autoren erklären die Verringerung des Widerstands bei kleinen Elektrodenabständen damit, dass Oberflächenstufen bei kleinen Abständen nur eine untergeordnete Rolle spielen. Zusätzlich zeigt der blaue Bereich das erwartete Verhalten des Widerstands für einen zusätzlichen 3DTransportkanal durch das Volumensilizium. Inset (a-c) zeigt schematisch den zugrundeliegenden Prozess. Die Abweichung vom experimentellen Verhalten ist ein erneuter Hinweis auf ausschließlichen 2D-Transport durch den $S_{1}$-Zustand. (b) Vierpunktmessung mit $8 \mu \mathrm{m}$ Elektrodenabstand auf einem Wafer mit „Stepbunching“. Ein erhöhter Widerstand wird über „Stepbunching“-Regionen gefunden. Die Autoren schlussfolgern daher, dass eine Streuung oder Transmission an Stufen einen großen Beitrag zur makroskopisch bestimmten Leitfähigkeit hat [107].

II. Mit derselben Methode wurde die Leitfähigkeit von Substratstufen, die zu „Stepbunching-Bereichen“ koaleszieren wurden, bestimmt. Hierbei wurde im Wesentlichen die Differenz der Leitfähigkeiten auf den Terrassen und über „Stepbunching“-Bereichen verwendet. Mit Hilfe dieser zweiten Methode wurde die Leitfähigkeit über Substratstufen hinweg zu $\sigma_{\text {Stufe }}=4000 \Omega^{-1} \mathrm{~m}^{-1}$ bestimmt.

III. Im Gegensatz zu den zwei o. g., über viele Stufen mittelnden Verfahren, bestimmten die Autoren $\sigma_{\text {Stufe }}$ auch rein mikroskopisch. Hierzu wurde die mit dem STM vermessene Phasenlage von Friedel-Oszillation an Stufenkanten analysiert. Dazu wird der Transport über die Stufenkante als quantenmechanische Transmission über eine Barriere aufgefasst. Die Wellenfunktionen des $\mathrm{S}_{1}$-Zustands werden an der Barriere 
reflektiert und bilden ein stehendes Interferenzmuster in der LDOS, was als FriedelOszillation bezeichnet wird. Die Phasenlage $\Phi$ der Friedel-Oszillation ist dabei direkt verknüpft mit der Transmission $\mathrm{T}$ über die Barriere zu $T=1-\left(1+\tan ^{2}(\Phi+\pi)\right)^{-1}$. Die Barriere wird als $\delta$-Barriere der Stärke $10 \mathrm{eV} \AA$ modelliert. Bei einer geschätzten Barrierendicke von einer atomaren Bindungslänge des Si-Volumenkristalls von 2,35 Å entspricht dies einer Barrierenhöhe von 4,5 eV. Eine energieunabhängige Transmission von $(30 \pm 15) \%$ wurde bestimmt. Dies bedeutet, dass Elektronen mit einer Wahrscheinlichkeit von 30\% die Stufe passieren können. Mit Hilfe von $\mathrm{k}_{\mathrm{f}}$ aus Literaturwerten bestimmten Matsuda et. al. die mikroskopische Leitfähigkeit über Substratstufen im Rahmen der Beschreibung nach Landauer (siehe hierzu auch Kapitel 1.2.2.2) $\mathrm{zu} \sigma_{\text {Stufe }}=(9000 \pm 4000) \Omega^{-1} \mathrm{~m}^{-1}$.

Zusammenfassend verstehen die Autoren den Transport über eine monoatomare Substratstufe als quantenmechanische Transmission über eine Barriere. Im Rahmen der LandauerTransportbeschreibung wird der Transmissionskoeffizient von $30 \%$ in eine Leitfähigkeit über die Substratstufen überführt (siehe hierzu auch Abschnitt 1.2.2.2). Als Mittelwert der drei komplementären Analysen spezifizieren sie die Stufenleitfähigkeit zu $\sigma_{\text {Stufe }}=5000 \Omega^{-1} \mathrm{~m}^{-1}$. $\sigma_{\text {Stufe }}$ ist hierbei als Leitfähigkeit eines ballistischen 2D-Systems mit einem 1D-Liniendefekt zu verstehen. Abbildung 37 (a) verdeutlicht dies schematisch.

Eine Defektlinie durchkreuzt ein ballistisches 2D-System. Die Leitfähigkeit wird über die strominjizierenden Kontakte bestimmt. Die Messung ist somit eine Zweipolmessung und beinhaltet auch die Kontaktwiderstände [39]. Ein System der Breite b $=(1 \mathrm{~m}, 1 \mathrm{~mm}, 1 \mu \mathrm{m}, 1$ $\mathrm{nm})$ und einer atomaren Substratstufe hat demnach eine Leitfähigkeit von $5000 \Omega^{-1}, 5 \Omega^{-1}, 5$ $10^{-3} \Omega^{-1}$ bzw. $510^{-6} \Omega^{-1}$. Der Widerstand über Substratstufen spielt im Makroskopischen zwar eine untergeordnete Rolle, im Mikroskopischen kann er jedoch einen starken Einfluss auf $z$. B. die Verteilung lokaler Strompfade ausüben. 


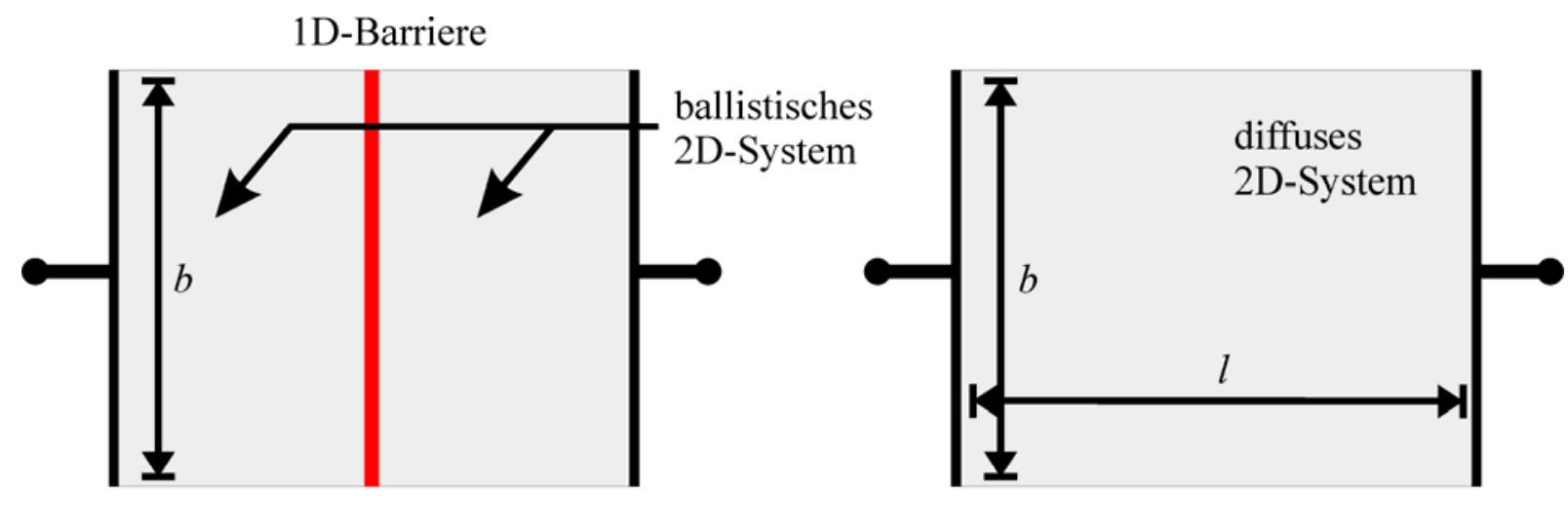

(a)

(b)

Abbildung 37: Schemaskizze zum Verständnis der Dimension von $\sigma_{\text {Stufe }}\left[\Omega^{-1} m^{-1}\right]$ und $\sigma_{2 D E G}\left[\Omega^{-1} / \square\right]$. (a) Ein ballistisches 2D-System der Breite b wird durch eine 1D-Defektlinie gestört. Die Leitfähigkeit des Systems wird in Zweipolgeometrie bestimmt, d.h. inklusive der Kontaktwiderstände. Der Leitwert skaliert mit $G_{\text {System }}=\sigma_{\text {Stufe }} \bullet b$. (b) Ein diffuses 2D-System mit einer spezifischen Leitfähigkeit von $\sigma_{2 D E G}\left[\Omega^{-1} / \square\right]$ skaliert mit $G_{S y s t e m}=\sigma_{2 D E G} \bullet b / l$ und ist damit bei festem Längen-Breiten Verhältnis größeninvariant.

Abbildung 37 (b) illustriert die Messung der Leitfähigkeit an einem diffusen 2D-System der Breite $b$ und Länge 1. Der Leitwert dieses 2D-Systems ist im Gegensatz zur Leitfähigkeit inklusive 1D-Defekt unabhängig von der quadratischen Systemgröße und skaliert mit $\mathrm{G}_{\text {System }}=\sigma_{2 \mathrm{DEG}} \bullet \mathrm{b} / \mathrm{l}$. Seine spezifische Leitfähigkeit $\sigma_{2 \mathrm{DEG}}$ hat die Einheit $\left[\Omega^{-1}\right]$. Um Verwechslungen mit dem Leitwert des Systems zu vermeiden, wird die Leitfähigkeit im Rahmen dieser Arbeit mit $\left[\Omega^{-1} / \square\right]$ bezeichnet, um anzudeuten, dass es sich um eine Sheet-Leitfähigkeit handelt. 


\subsubsection{Zusammenfassung}

Die publizierten Leitfähigkeiten durch den Oberflächenzustand der $\operatorname{Si}(111)(\sqrt{3} \times \sqrt{ } 3)-A g$ Rekonstruktion schwanken zwischen (20...3000) $\mu \mathrm{S} / \square$, wobei tendenziell größere Leitfähigkeiten bei kleinerem Vierpunkt-Elektrodenabstand zu finden sind. Die Leitfähigkeit durch die Raumladungszone, welche als Kurzschlusskanal für den Ladungstransport durch das 2DEG fungieren würde, wird auf n-dotiertem Silizium zu maximal $\sim 50 \mu \mathrm{S} / \square$ abgeschätzt.

Der Ladungstransport durch das 2DEG wird durch Substratstufen des Si(111)-Wafers behindert. Der Transport über die Stufen wird als Tunneln über eine quantenmechanische Barriere aufgefasst. Die Transmission über eine Stufe wird zu (30 15$) \%$ bestimmt. Es wird vermutet, dass sich die makroskopisch gemessene Leitfähigkeit aus der lokalisierten Streuung an Substratstufen und den diffusen Streuprozessen zusammensetzt.

Bei einer Besetzung entsprechend $\mathrm{E}_{\mathrm{f}} \sim 350 \mathrm{meV}$ wird die Leitfähigkeit über Stufenkanten durch die Auswertung von LDOS-Oszillationen $\mathrm{zu} \sigma_{\mathrm{Stufe}}=(9000 \pm 4000) \Omega^{-1} \mathrm{~m}^{-1}$ spezifiziert [114]. Studien an Ensembles von Stufenkanten durch Vierpunktmessungen bestimmen die mittlere Leitfähigkeit einer Stufenkante zu $\sigma_{\text {Stufe }}=(3000 \ldots 4000) \Omega^{-1} \mathrm{~m}^{-1}$, so dass die Autoren von einer Stufenleitfähigkeit von $\sigma_{\text {Stufe }}=(5000) \Omega^{-1} \mathrm{~m}^{-1}$ ausgehen [114]. 


\subsection{Raster-Tunnel-Potentiometrie auf dem natürlichen Perko- lationsnetzwerk der $\operatorname{Si}(111)(\sqrt{3} \times \sqrt{3})$-Ag Rekonstruktion}

Abbildung 38 zeigt einen im Potentiometrie-Modus aufgenommen Rohdatensatz auf der $\operatorname{Si}(111)(\sqrt{3} \times \sqrt{3})-A g$ Rekonstruktion bei Raumtemperatur. Im Umkehrpunkt zwischen der Hin- und Rückspur einer Rasterzeile wurde die Querstromrichtung umgedreht. Man erhält dadurch zeitlich eng auf einander folgende Potentialdaten von zwei Querstromrichtungen nahezu gleichen Betrags. Der zeitliche Unterschied zwischen der Hinspur ( $j_{\text {extern }} \rightarrow$ ) und der Rückspur $\left(\mathrm{j}_{\mathrm{extern}} \leftarrow\right)$ liegt im Bereich von einigen Sekunden, so dass Driftartefakte sowie Effekte durch Kontaktdegradation vermindert werden. Potentialdaten beider Querstromrichtungen einer Rasterzeile lassen sich auf diese Weise direkt miteinander vergleichen. Aufgrund der bis zu mehreren Stunden langen Messzeit zur Aufnahme eines kompletten Datensatzes wird die langsame Rasterrichtung (vertikale Richtung) im Allgemeinen stark von Driftartefakten beeinflusst.
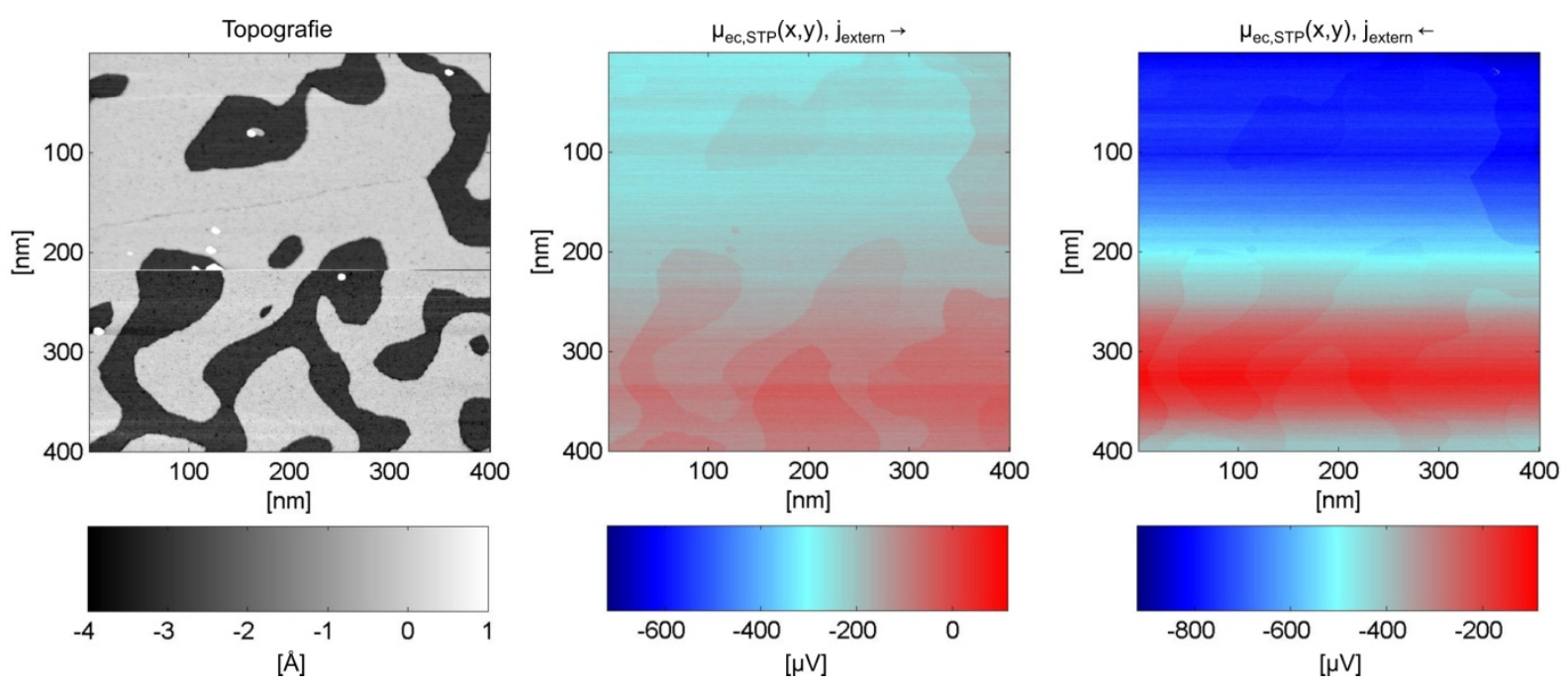

Abbildung 38: (a) Nachbearbeitete STM-Topographie der Si(111)( $\sqrt{3} x \sqrt{3})$-Ag Rekonstruktion (400x400 $\mathrm{nm}^{2}$ bei einer Auflösung von $10^{6}$ Messpunkten, $U_{\text {Bias }}=-70 \mathrm{mV}, \mathrm{I}_{t}=1 \mathrm{nA}$ ). (b) $\mu_{e c, S T P}(x, y)$ Rohdaten (Hinspur) bei einer mittleren makroskopischen Stromdichte von $38 \mathrm{~mA} / \mathrm{m}$. (c) $\mu_{e c, S T P}(x, y)$ Rohdaten (Rückspur) bei einer mittleren makroskopischen Stromdichte von -26 mA/m. Die makroskopische Stromrichtung wurde im Vergleich zu (b) invertiert. Ein starkes Driftsignal ist den $\mu_{e c, S T P}$ Daten überlagert. Obwohl die Daten für beide Stromrichtungen aufeinanderfolgend aufgenommen wurden, ist die Drift unterschiedlich. Die Messzeit für diesen Datensatz betrug ca. 9 Stunden. 


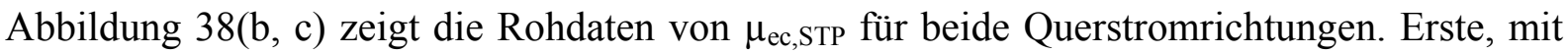
der Topographie korrelierte Potentialverläufe, lassen sich erkennen. Die Potentialdaten erscheinen entlang der langsamen Rasterrichtung stark driftbehaftet. Beide Querstromrichtungen zeigen dabei den gleichen Trend, allerdings mit unterschiedlicher Stärke.

Zur besseren Darstellung illustriert Abbildung 39 das mittlere Potential jeder Rasterzeile. Obwohl die Potentialdaten beider Stromrichtungen simultan aufgenommen worden sind, besitzen sie eine stark unterschiedliche Drift. Die Stärke des maximalen Driftsignals lässt sich $\mathrm{zu} \sim 40 \mathrm{nV} / \mathrm{s}$ abschätzen.

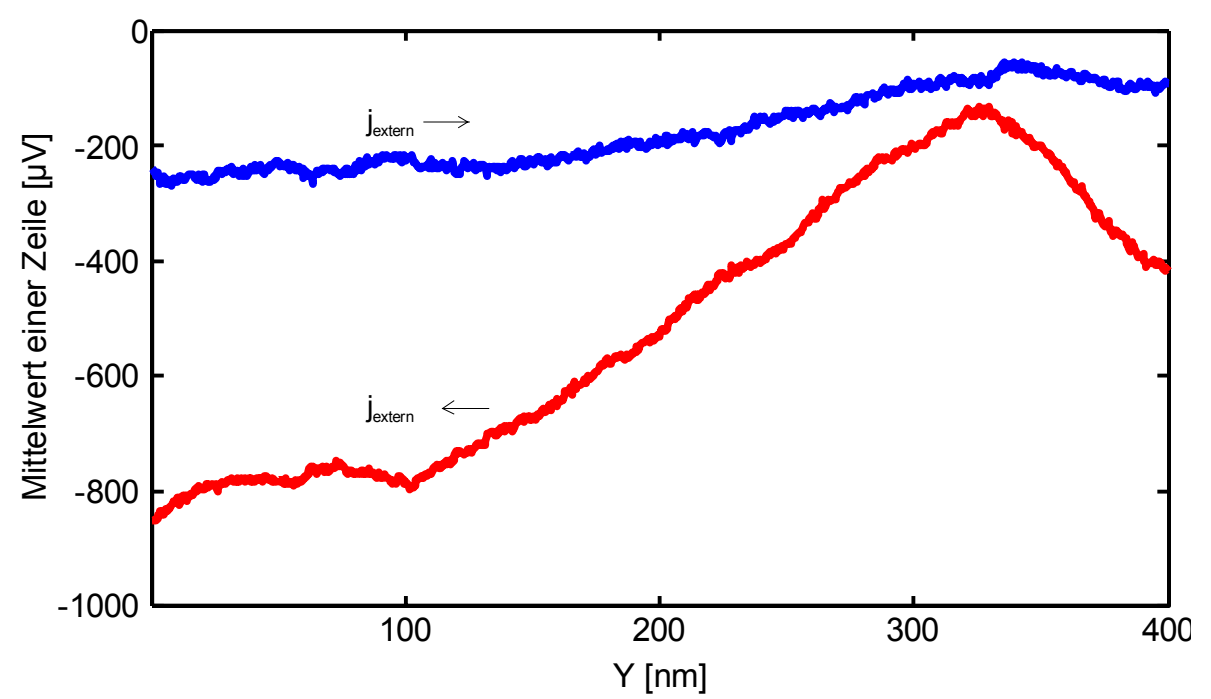

Abbildung 39: Das mittlere Potential einer jeden Zeile aus Abbildung 38(b, c) als Funktion der vertikalen Länge. Obwohl die Daten für beide Stromrichtung aufeinanderfolgend

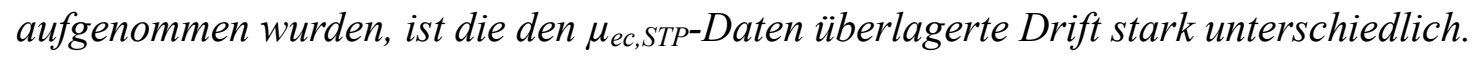

Um die gemessenen Potentialdaten einer weiteren Analyse zugänglich zu machen, ist es unabdingbar, einen Linienabzug entlang der langsamen Rasterrichtung durchzuführen. Hierbei wird den Potentialwerten einer Zeile der entsprechende Mittelwert abgezogen. Die so veränderten Potentialdaten enthalten damit streng genommen keine Information mehr über den vertikalen Potentialverlauf. Den durch Linienabzug nachbearbeitet STP-Datensatz zeigt Abbildung 40. 

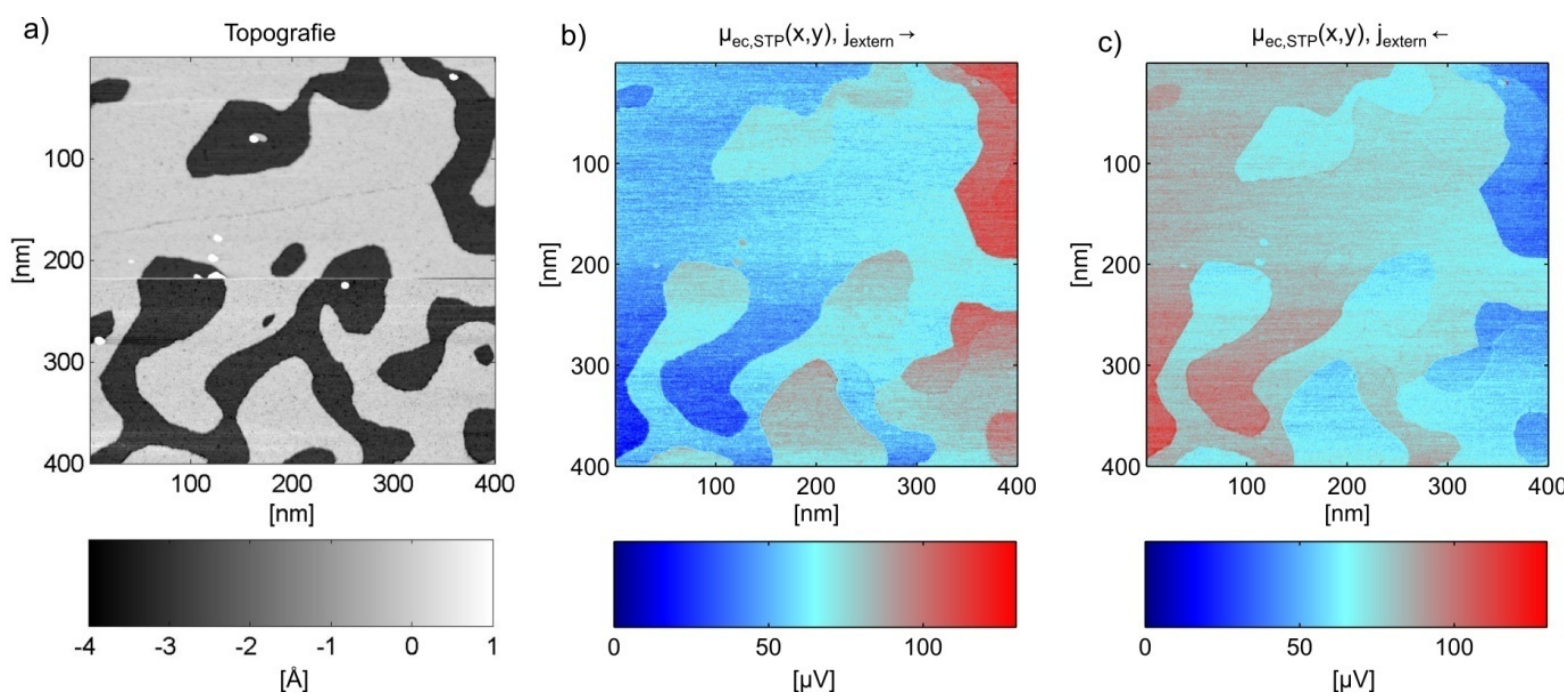

Abbildung 40: Nachbearbeiteter Datensatz aus Abbildung 38: Der Mittelwert einer jeden Rasterzeile wurde von den $\mu_{e c, S T P-D a t e n}$ abgezogen.

Auf den freien Flächen sind entlang der vertikalen Rasterrichtung nichtmonotone Potentialverläufe erkennbar. Dieses sind Artefakte des Linienabzugs. Um sie zu beseitigen wurde ein stückweiser, iterativer Linienabzug angewendet. Abbildung 41 illustriert die verschiedenen Bearbeitungsschritte. Zuerst wird ein Linienabzug auf Basis der Werte des schmalen Rechtecks durchgeführt. Abbildung 41(a) zeigt das Ergebnis. Die Vertiefung in der linken oberen Ecke führt zu einem Streifen falscher Werte, die wiederum mit einem Linienabzug innerhalb eines weiteren Rechtecks angepasst werden (siehe (b)). Jede Zeile dieses Streifens erhält zusätzlich noch einen Potentialoffsetwert, welcher zuvor im unteren Bereich des Rechtecks ermittelt und bereits durch den ersten Linienabzug erfasst worden ist. Das Ergebnis einer weiteren Nachbearbeitung im unteren Teil zeigt Abbildung 41(c). Bevor auf einzelne Beobachtungen im Detail eingegangen wird, erscheint es zweckmäßig, die PotentiometrieDaten zusammen mit den Topographie-Daten als 3D-Darstelllung aufzubereiten. Die Topographie wird hierbei als Höheninformation dargestellt, der die Potentiometrie-Daten farblich überlagert sind. 

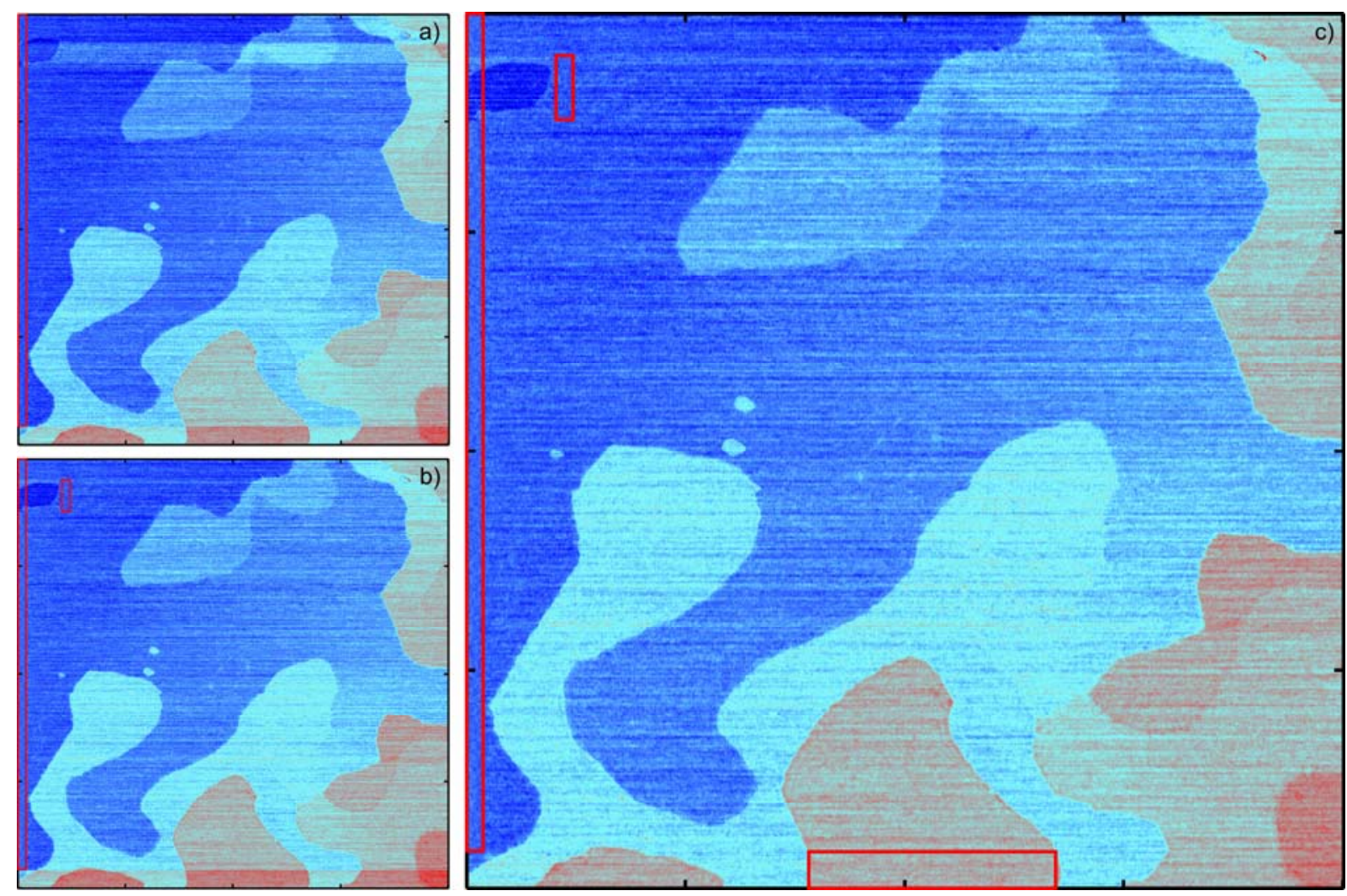

Abbildung 41: Darstellung der verschiedenen Bearbeitungsschritte zum Entfernen der Drift. (a) Entlang des Rechtecks wurde ein Linienabzug durchgeführt. (b) Reparatur des oberen Streifens: Die untere Zeile des Rechtecks überlappt sich mit dem sinnvollen Bearbeitungsbereich aus der vorherigen Bearbeitung. Der Mittelwert dieser Zeile wird daher als Offset des Linienabzugs im kleinen Rechteck verwendet. (c) Entsprechend wird der untere Teil repariert, indem die obere Zeile des Rechtecks als Offset für den Linienabzug des unteren Streifens verwendet wird. Das sich ergebende Potentialbild ist frei von unphysikalischen, nicht monotonen Verläufen. 

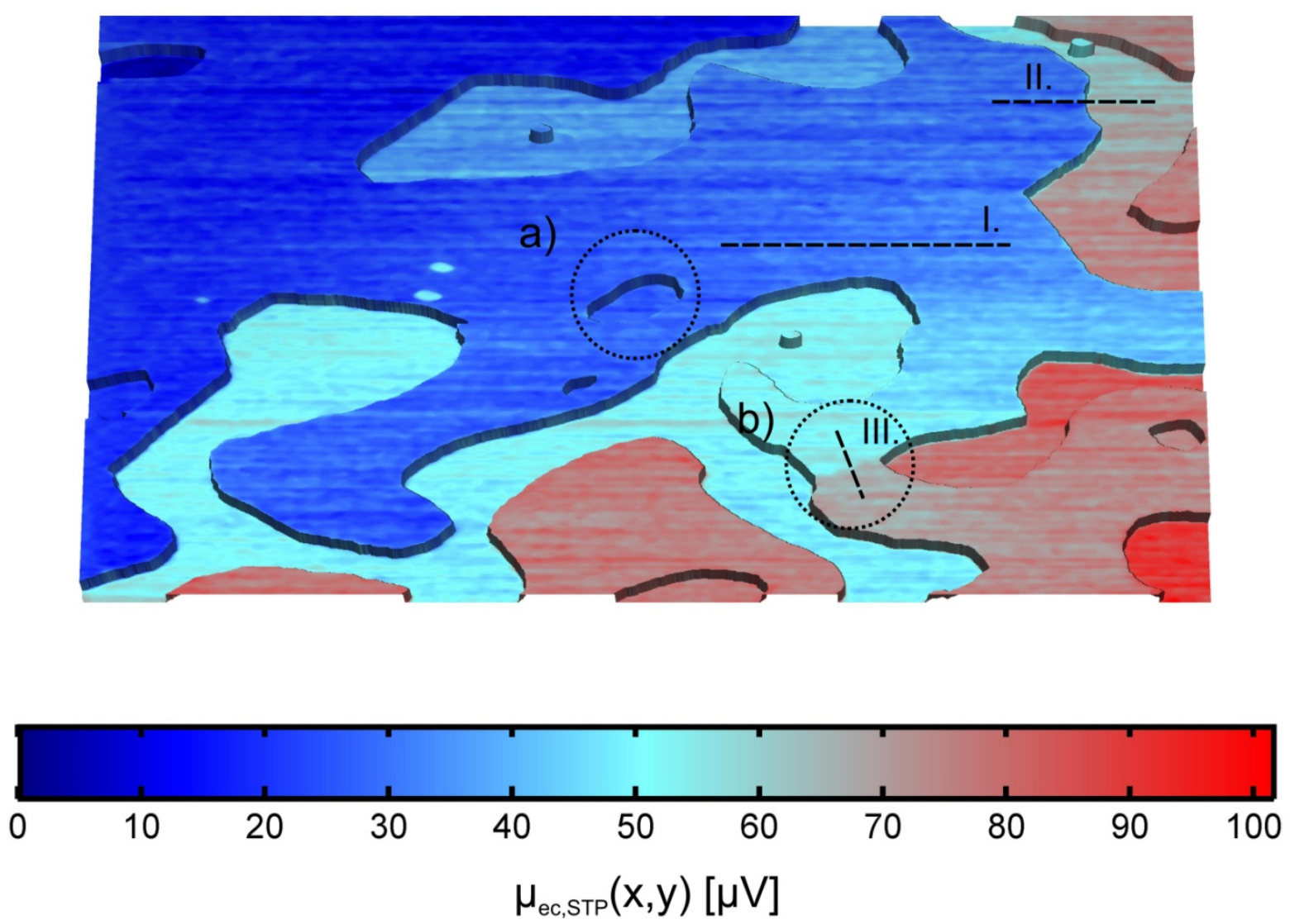

Abbildung 42: 3D-Darstellung der STP Daten (400 x $\left.400 \mathrm{~nm}^{2}\right)$ aus Abbildung 40: Die Topographie wurde auf die Höhe einer monoatomaren Si(111)-Stufe binarisiert und ist als Höheninformation dargestellt. Die $\mu_{e c, S T P-D a t e n}$ sind der Topographie farbkodiert überlagert. Gradienten auf den freien Flächen und lokalisierte Sprünge in $\mu_{e c, S T P}$ an monoatomaren Terrassenstufen sind ersichtlich. (a) kleine eingeschlossene Strukturen liegen auf demselben Potential wie ihre Umgebung. (b) zeigt den Potentialabfall an einer Domänengrenze. Die gestrichelten Linien zeigen die Position der Linienprofile aus Abbildung 43.

Abbildung 42 illustriert, dass die Potentiallandschaft eindeutig mit der Topographie korreliert ist. Sie erinnert an ein Perkolationsnetzwerk, das durch die Terrassenstufen geformt wird. Die Stromdichte wird in einem solchen Fall stark inhomogen und ortsabhängig sein. Abhängig von der jeweiligen Lage einer Stufe im Perkolationsnetzwerk finden sich lokalisierte Potentialsprünge von bis $\mathrm{zu} \sim 40 \mu \mathrm{V}$.

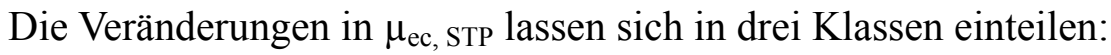

1.Homogene Potentialabfälle entlang der freien Fläche (Linienprofil Bereich I).

2.Lokalisierte Potentialsprünge an Stufenkanten (Linienprofil Bereich II).

3. Lokalisierte Potentialsprünge an Domänengrenzen (Linienprofil Bereich III). 
Ein Linienprofil auf der freien Fläche, d.h. aus Bereich I zeigt Abbildung 43 (a). Das Linienprofil wurde entlang der schnellen Rasterrichtung evaluiert, so dass Artefakte durch die Bildbearbeitung ausgeschlossen werden können. Im Rahmen der experimentellen Auflösung wird ein linearer Potentialgradient von ca. $20 \mu \mathrm{V}$ auf $100 \mathrm{~nm}$ beobachtet. Dies entspricht einem elektrischen Feld von $200 \mathrm{~V} / \mathrm{m}$. Der Ladungstransport wird in diesen Bereichen maßgeblich diffus stattfinden und ist durch delokalisierte Streuung (Elektron-Elektron- und Elektron-Phonon-Streuung) charakterisiert. Eine lokalisierte Streuung an statisch verteilten atomaren Defekten lässt sich jedoch auflösungsbedingt nicht eindeutig ausschließen. Unter der sicherlich fraglichen Annahme, dass die im Mittel extern aufgeprägte Querstromdichte von $38 \mathrm{~mA} / \mathrm{m}$ durch diese Fläche fließt, wird die Leitfähigkeit des $2 \mathrm{DEG}$ nach $\sigma=j / \mathrm{E}$ zu

$$
\sigma_{2 D E G}=1900 \mu S / \square \text { berechnet. }
$$

Es wird sich im weiteren Verlauf zeigen, dass dieser Wert nur um etwa 50\% von dem einer wesentlich genaueren Bestimmung der 2DEG-Leitfähigkeit abweicht (siehe Kapitel 3.2.2.2).

Den Potentialverlauf über eine Stufenkante hinweg zeigt Abbildung 43 (b). Das Potential variiert am Ort der Stufe um $40 \mu \mathrm{V}$ innerhalb von etwa $2 \mathrm{~nm}$. Eine direkte Bestimmung der Stufenleitfähigkeit aus den Potentiometrie-Daten wird in Kapitel 3.2.1 durchgeführt. Dem räumlichen Verlauf von $\mu_{\mathrm{ec}, \text { STP }}$ am Ort der Stufenkante widmet sich detailliert Kapitel 4. Das Linienprofil über eine Domänengrenze zeigt Abbildung 43 (c). Ob es sich um eine Antiphasengrenze oder eine Zwillingskorngrenze handelt, ist auflösungsbedingt nicht eindeutig zu verifizieren. Das Potential variiert am Ort der Domänengrenze um ca. $10 \mu \mathrm{V}$. Die Variation ist allerdings nicht so lokalisiert wie am Ort der Stufe. Dies könnte darin begründet sein, dass das Linienprofiel nicht entlang der schnellen Rasterrichtung evaluiert werden kann. Zunächst wird die Variation in $\mu_{\mathrm{ec}, \text { STP }}$ als Funktion der externen Querstromdichte analysiert. Abbildung 44 zeigt eine weitere Messung bei der ein Finger des Perkolationsnetzwerkes bei vier verschiedenen Querstromdichten abgebildet worden ist. Die Auswertung ist in Abbildung 45 dargestellt. 


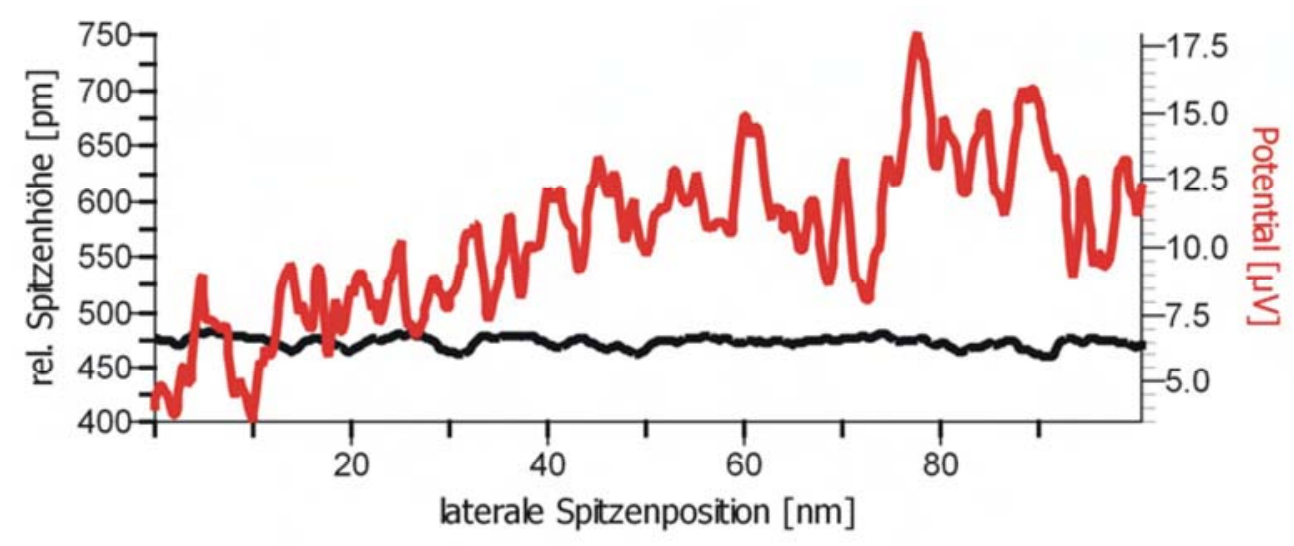

(a) Potentialabfall auf freier Fläche

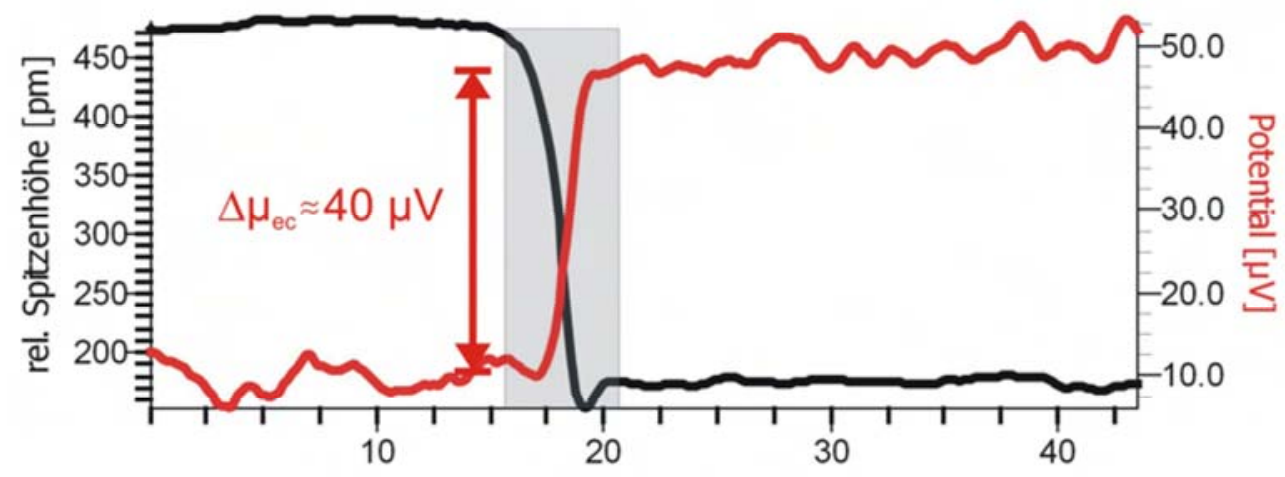

laterale Spitzenposition $[\mathrm{nm}]$

(b) Potentialabfall an Stufenkante

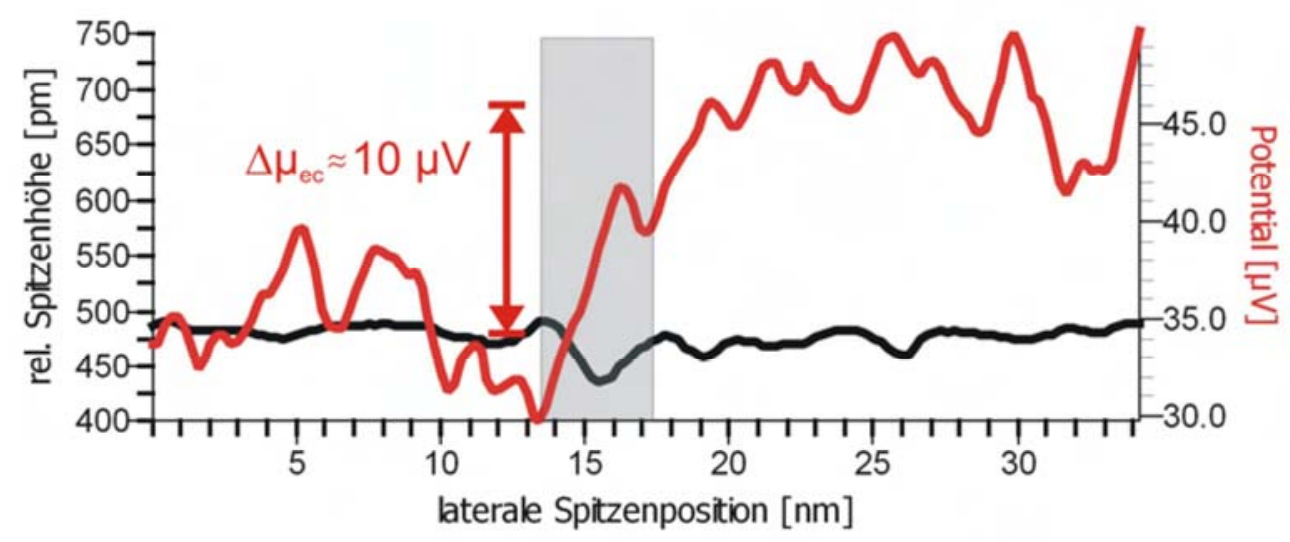

(c) Potentialabfall an Domänengrenze

Abbildung 43: Linienprofile gemäß der gekennzeichneten Bereiche aus Abbildung $42\left(\mu_{e c, S T P}\right.$ (rot), Topographie (schwarz)). (a) Homogener Potentialgradient auf der freien Fläche, (b) lokalisierter Potentialsprung an einer Stufenkante und (c) Potentialsprung an einer Domänengrenze. Die Profile wurden über jeweils $4 \mathrm{~nm}$ gemittelt. Abbildung aus Ref. [36] 
a)

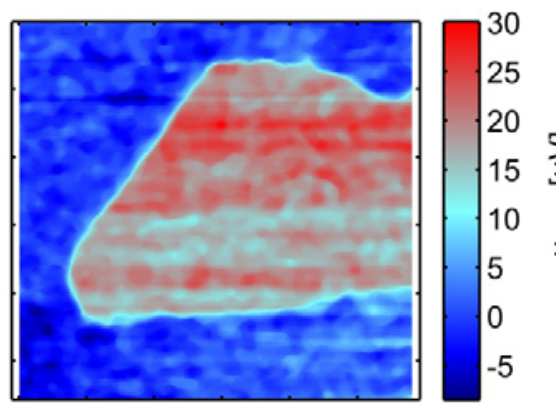

b)

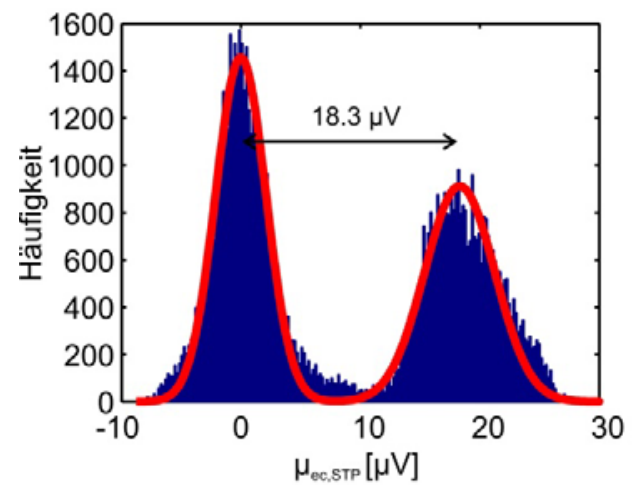

e)

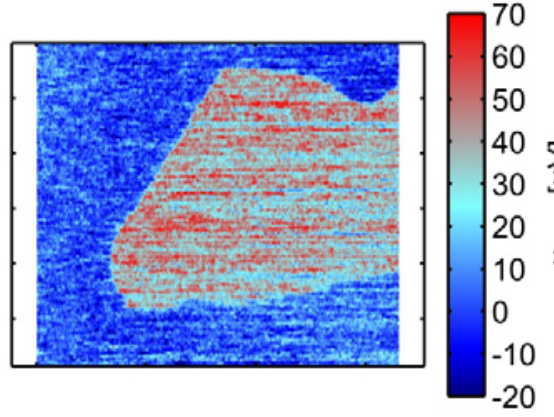

f)

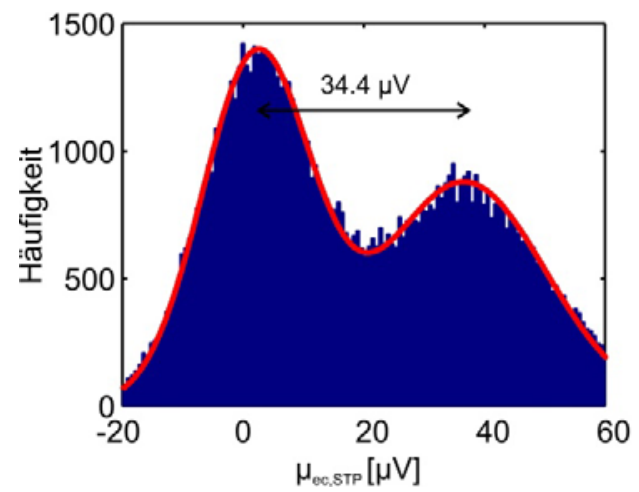

c)

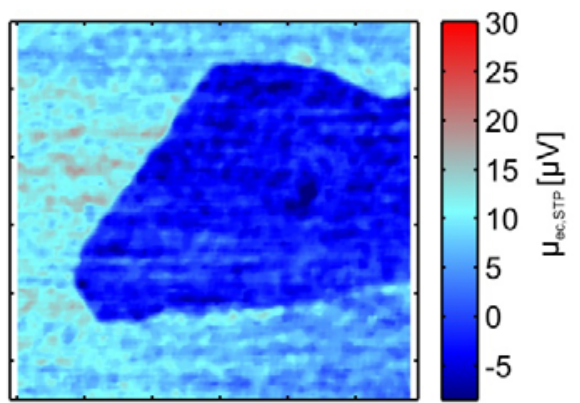

d)

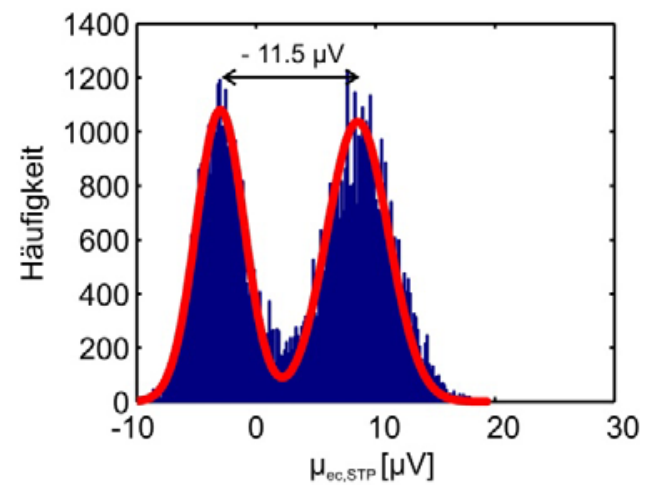

g)

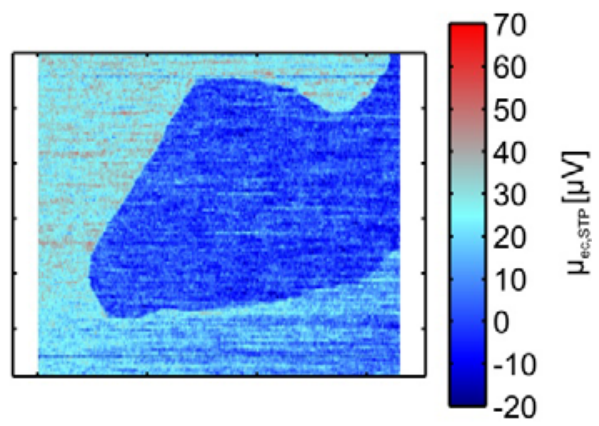

h)

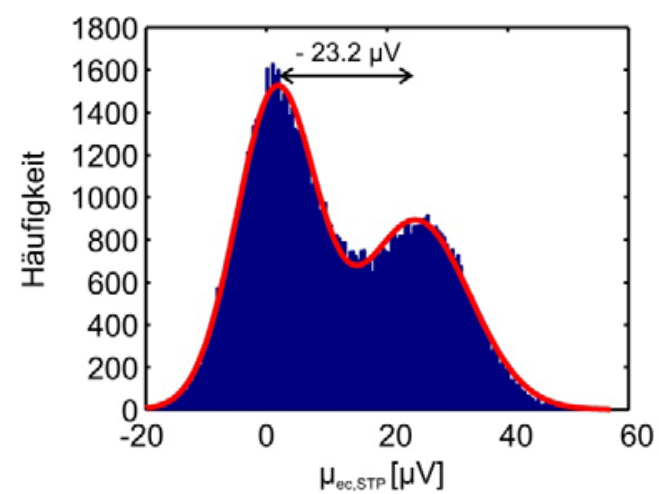

Abbildung 44: Analyse des Potentialsprungs an Stufenkanten bei verschiedenen externen, makroskopischen Querstromdichten von (a, b) $38 \mathrm{~mA} / \mathrm{m},(c, d)-26 \mathrm{~mA} / \mathrm{m},(e, f) 76 \mathrm{~mA} / \mathrm{m}$ und (g, h) $-52 \mathrm{~mA} / \mathrm{m}$. Das gemittelte elektrische Feld auf den freien Flächen wurde für diese Analyse abgezogen. 
Der Potentialsprung an der Stufenkante ist eine lineare Funktion der makroskopisch getrieben Querstromdichte. Für $\mathrm{I}_{\mathrm{q}}=0$ verschwindet die Potentialdifferenz (Abweichung $0.5 \mu \mathrm{V}$ ). Bei der betrachteten Struktur beträgt der Potentialsprung an einer Stufenkante $454(23) \mu \mathrm{V} \mathrm{m} / \mathrm{A}$.

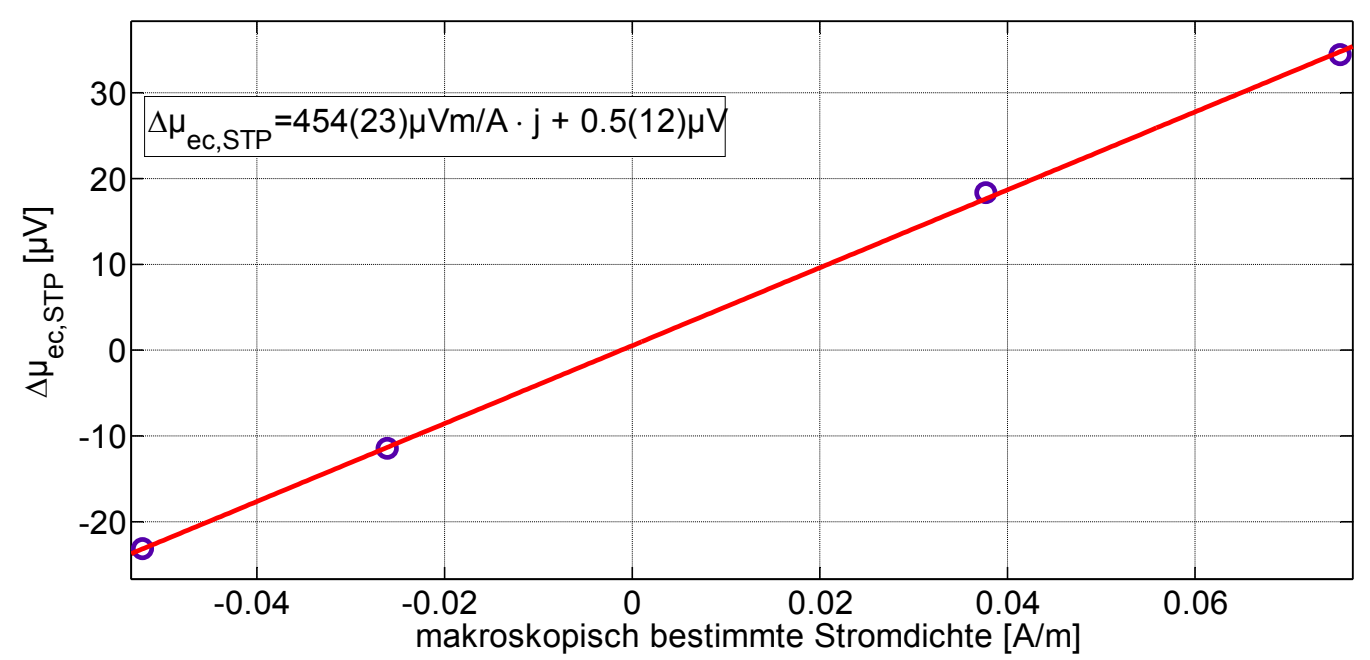

Abbildung 45: Zusammenfassung der Analyse aus Abbildung 44. Der Potentialsprung an einer monoatomaren Terrassenstufe ist eine lineare Funktion der makroskopisch getriebenen Querstromdichte.

Die Transmission der Stufe ist demnach für Transportvorgänge energieunabhängig. Schätzt man die Barrierenhöhe nach Abschnitt 3.1.2 mit ca. 4,5 eV ab, so ist dieses Verhalten leicht zu verstehen, da eine Veränderung der effektiven Barrierenhöhe bei den betrachteten Stromdichten in der Größenordnung von $\Delta \mu_{e c} / 4,5 \mathrm{eV} \approx 10^{-5}$ liegt. Eine energieunabhängige Transmission wird ebenfalls durch die Analyse von Matsuda et. al. bestätigt [114]. 


\subsubsection{Mikroskopische Bestimmung der Stufenleitfähigkeit}

Im vorangegangenen Kapitel wurde experimentell gezeigt, dass der mikroskopische Spannungsabfall an einer Terrassenstufe proportional zum extern aufgeprägten Strom ist. Der entsprechende Spannungsabfall lässt sich demnach linear mit der mikroskopischen Stromdichte über eine spezifische Stufenleitfähigkeit verknüpfen $\Delta \mu_{\text {Stufe }}=\sigma_{\text {Stufe }}^{-1} j_{\text {stufe }}$ (siehe hierzu auch Abschnitt 3.1.2). Das Problem bei dieser Art der mikroskopischen Stufenleit-

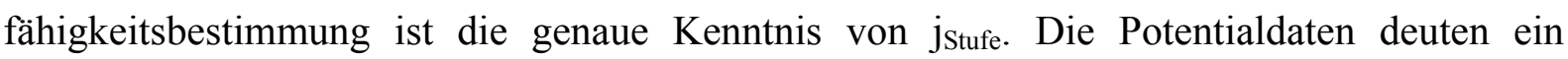
Perkolationsnetzwerk an, so dass die Stromdichte durchaus sehr inhomogen sein kann.

Um dennoch eine experimentelle, mikroskopische Abschätzung der Stufenleitfähigkeit machen zu können wird davon ausgegangen, dass es im globalen Maßstab keine perkolierenden, d.h. kurzgeschlossenen Strompfade gibt. Perkolationspfade hoher Stromdichte müssen demnach irgendwo eine Terrassenstufe überwinden. Sie verursachen an diesen Stellen einen großen Potentialsprung, der mit einem sehr kleinen Potentialsprung in Gebieten geringer Stromdichte einhergeht. Eine Aussage über die Stufenleitfähigkeit ist in diesem Fall möglich, wenn man die lokalen Stromdichten und Spannungsabfälle am Ort der Stufe durch ihre Mittelwerte ersetzt. Als Mittelwert des Stroms wird die extern aufgeprägte Querstromdichte verwendet. Der mittlere Spannungsabfall kann aus großskaligen STP-Messungen extrahiert werden.

Abbildung 46 zeigt entsprechende STP-Daten. Um statistisch signifikante Mengen an Potentialsprüngen analysieren $\mathrm{zu}$ können, wurde die Auswertung automatisiert. Die Positionen der Terrassenstufe wurden aus der Topographie mit Hilfe einer „Kantensuchroutine“ pixelgenau extrahiert. Um Driftartefakte zu vermeiden, wurden an diesen Positionen nur Schnitte entlang der schnellen Rasterrichtung, welche mit der externen Querstromrichtung übereinstimmt, analysiert. An den gemessenen Potentialsprüngen in $\mu_{\mathrm{ec}, \mathrm{STP}}(\mathrm{x})$ wurde eine symmetrischen Stufenfunktion der Form $\mu_{e c, S T P}(x)=\Delta \mu_{\text {Stufe }} \cdot\left(1+\exp ^{\frac{\mathrm{x}-\mathrm{x}_{0}}{\Delta \mu}}\right)^{-1}+\mu_{\text {Offset }}$ angepasst.

Die Verteilung aller Potentialsprünge $\Delta \mu_{\text {Stufe }}$ in den entsprechenden STP-Daten zeigen die Histogramme (b, d). Der STP-Datensatz (a, b) zeigt die Potentiallandschaft sowie die Potentialsprünge aus einem Probenbereich von 400 x $400 \mathrm{~nm}^{2}$. Die Finger des Perkolationsnetzwerks stellen in X-Richtung sowohl abfallende als auch ansteigende Potentialsprünge dar. Entsprechend finden sich im Histogramm Potentialsprünge, die der externen Stromrichtung 
entgegengesetzt sind. Der Mittelwert aller Potentialsprünge $\Delta \mu_{\text {Stufe }}$ ist jedoch $7.3 \mu \mathrm{V}$ und entspricht vom Vorzeichen her der externen Querstromrichtung.

a)

c)
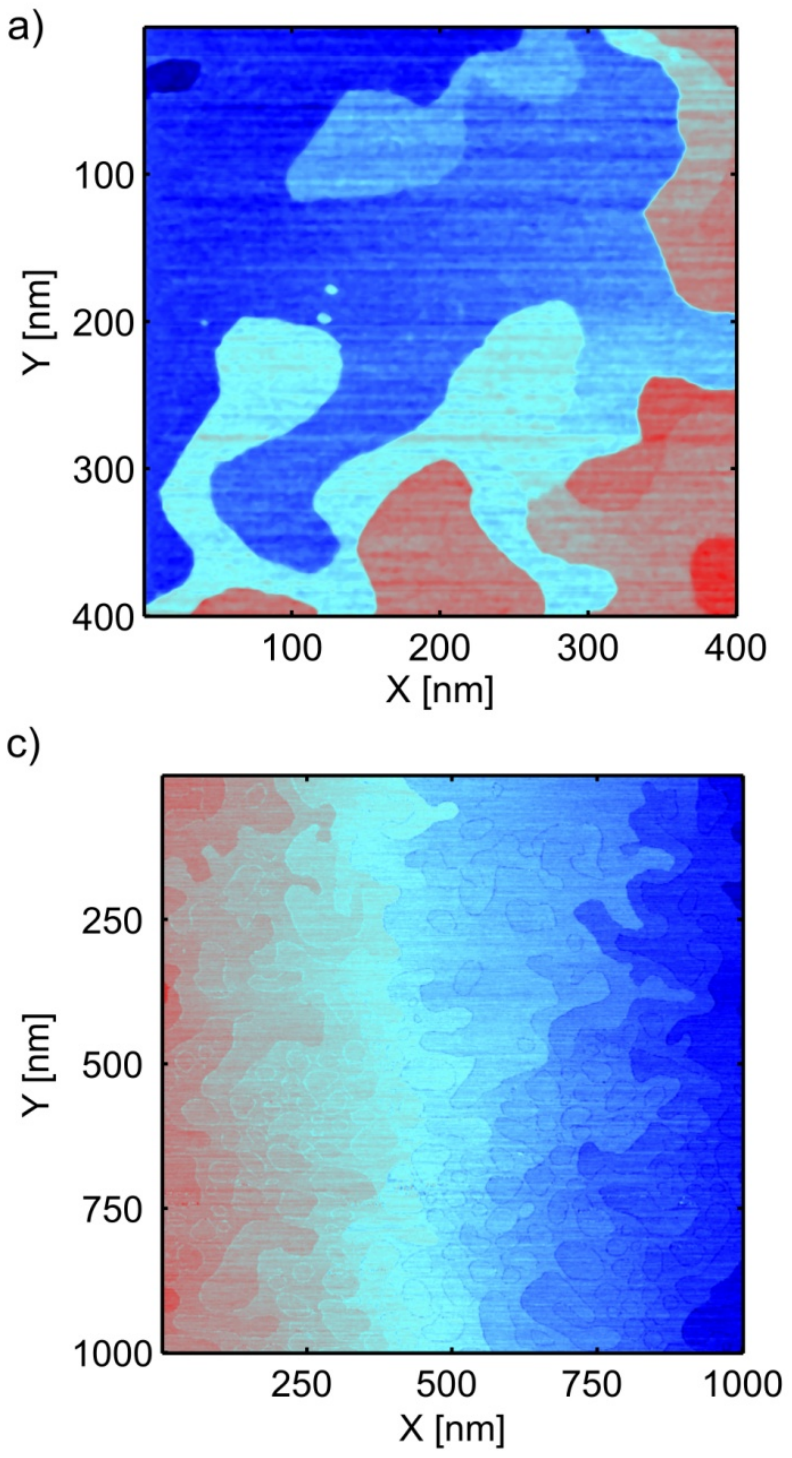

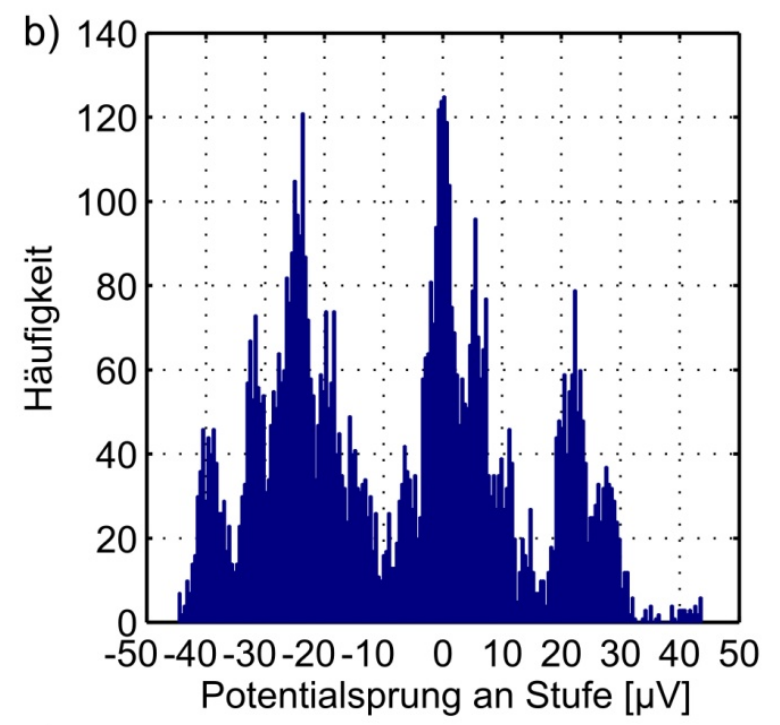

d)

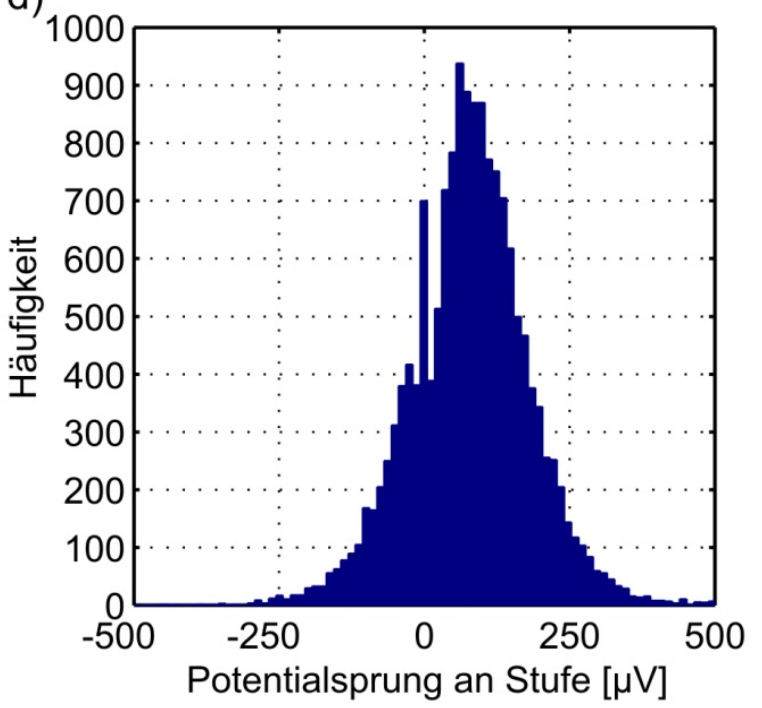

Abbildung 46: Analyse der Potentialsprünge über Stufenkanten: $(a, c)$ zeigen die zugrundeliegenden Potentiometrie-Bilder bei einer Querstromdichte von $j_{\text {extern }}=38 \mathrm{~mA} / \mathrm{m}$ und von $j_{\text {extern }}=450 \mathrm{~mA} / \mathrm{m}$. (b) Zeigt ein Histogramm der Potentialsprünge über Stufenkanten aus (a). Der Mittelwert der Potentialsprünge beträgt $7.3 \mu \mathrm{V}$. (d) Illustriert ein Histogramm der Potentialsprünge über Stufenkanten aus (c). Der Mittelwert der Potentialsprünge beträgt $72 \mu \mathrm{V}$ mit einer Standardabweichung von $79 \mu \mathrm{V}$. Aufgrund der hohen Stromdichte ist in (c) ein leichtes Thermospannungssignal an den Stufenkanten zu erkennen. 
Der extern aufgeprägte Strom fließt jedoch auch durch das Volumen des Si-Wafers. Es erscheint an dieser Stelle sinnvoll, den Wafer-Leitwert $G_{\text {Wafer }}=(16 \ldots 22 \mathrm{k} \Omega)^{-1}$ heraus zurechnen. Der Leitwert der $\operatorname{Si}(111)(\sqrt{3} \times \sqrt{3})$-Ag Rekonstruktion wurde in Kapitel 2.3.1 auf

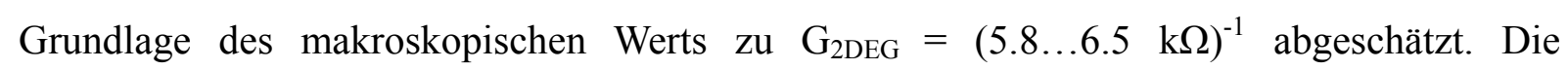
Stromdichte durch das 2DEG, bereinigt durch die parasitäre Leitfähigkeit des Wafers, ist demnach $j_{2 D E G}=j_{\text {extern }} \frac{G_{2 D E G}}{G_{2 D E G}+G_{\text {Wafer }}}$. Mit $\quad j_{2 D E G}=(70 \sim 80) \% \cdot j_{\text {extern }}$ ergibt $\quad$ sich die Stufenleitfähigkeit auf Basis der Auswertung aus Abbildung 46 (a, b) zu $\sigma_{\text {Stufe }}=(3600 \ldots 4200) \Omega^{-1} m^{-1}$. Dieser Wert darf jedoch nur als eine obere Grenze für die Leitfähigkeit verstanden werden, da nach (a) vermutet werden kann, dass der Querstrom einen Großteil der Terrassenstufen umfließen kann. Die Stromdichte durch die Stufe kann daher von

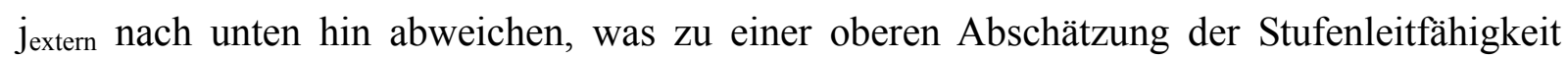
führt.

Größerskalige Abbildungen erlauben einen besseren Mittelungsprozess über diese Fehlerquellen. Abbildung 46 (c, d) zeigt die Auswertung auf $1 \times 1 \mu \mathrm{m}^{2}$ bestehend aus 16000 Einzelschnitten. Die Verteilung der Potentialsprünge ist mit einem Mittelwert von $72 \mu \mathrm{V}$ nun nahezu gaußverteilt. Mit der entsprechenden Standardabweichung und angenommener Normalverteilung ergibt sich der Mittelwert zu $\Delta \mu_{\text {Stufe }}=72 \pm 0.6 \mu V$. Die Stufenleitfähigkeit bei dieser Datenbasis ist $\sigma_{\text {Stufe }}=(4400 \ldots 5000) \Omega^{-1} m^{-1}$. Die nachfolgende Tabelle fasst den so bestimmten Wert mit Literaturwerten unter Benennung der experimentellen Methode zusammen.

\begin{tabular}{|l|l|l|}
\hline Referenz & Stufe & Methode \\
\hline Diese Arbeit & $<5000 \Omega^{-1} \mathrm{~m}^{-1}$ & $\begin{array}{l}\text { Mikroskopisch; wahre Transportmessung unter } \\
\text { Verwendung der externen Stromdichte }\end{array}$ \\
\hline Diese Arbeit & $(5000 \ldots 6800) \Omega^{-1} \mathrm{~m}^{-1}$ & $\begin{array}{l}\mathrm{E}_{\mathrm{f}}=250 \mathrm{meV} \text { aus STS; T=30\% aus Ref. [92] und } \mathrm{m}^{*} \text { aus } \\
\text { Literatur }\end{array}$ \\
\hline$[114]$ & $(9000 \pm 4000) \Omega^{-1} \mathrm{~m}^{-1}$ & Mikroskopisch; Phasenschub von Friedel-Oszillationen \\
\hline$[114]$ & $(3000 \ldots 4000) \Omega^{-1} \mathrm{~m}^{-1}$ & $\begin{array}{l}\text { Vierpunktmessungen; wahre Transportmessung; vizinale } \\
\text { Oberflächen bzw. „Stepbunching“-Bereiche }\end{array}$ \\
\hline
\end{tabular}


Eine erstaunlich gute Übereinstimmung mit bereits publizierten Literaturwerten ist anzumerken. Für die vorliegende Probe wird die Stufenleitfähigkeit zu $\sigma_{\text {Stufe }}=5000 \Omega^{-1} m^{-1}$ angenommen. Auf eine genaue Fehlerangabe wird an dieser Stelle aufgrund möglicher systematischer Fehlerquellen verzichtet. Im Gegensatz zu allen bisherigen Analysen, ist die Stufenleitfähigkeit in dieser Arbeit jedoch erstmalig mikroskopisch und in einem echten Transportexperiment bestimmt worden.

\subsubsection{Modellierung der Transporteigenschaften}

In Kapitel 3.2.1 wurde die spezifische Leitfähigkeit durch Stufenkanten zu $\sigma_{\text {Stufe }}=5000 \Omega^{-1} m^{-1}$ mikroskopisch bestimmt und als Leitwert eines ballistischen Systems mit einem 1D-Defekt inklusive der Dissipation an den Kontakten eingeführt. Dies ist jedoch eine stark idealisierte Vorstellung und im vorliegenden Fall sicherlich nicht richtig. Die homogenen Potentialgradienten auf den freien Flächen sind ein direktes Anzeichen einer diffusen Streuung. Eine zweidimensionale Modellierung der Transportvorgänge auf Grundlage der gemessenen Realstruktur erscheint schwierig und ist darüber hinaus auch konzeptionell nicht eindeutig. Daher wird im Folgenden der Transport durch die Realstruktur der $\operatorname{Si}(111)(\sqrt{3} \times \sqrt{3})$-Ag Rekonstruktion rein diffus modelliert.

Bis auf die Kontakte ist das System stromquellenfrei, so dass $\operatorname{div} \vec{j}_{\text {mikro }}(x, y)=0$ gilt. Ein Kurzschlusskanal durch die Raumladungszone unter dem 2DEG wird nach Abschnitt 3.1 vernachlässigt. Fern von lokalisierten Streuzentren folgt $\mu_{\mathrm{ec}}$ dem Verlauf des elektrostatischen Potentials (siehe Abschnitt 1.2.2), so dass bei der gewählten Modellierung des Ladungstransports das ohmsche Gesetz $\vec{j}(x, y)=\sigma(x, y) \nabla \mu_{e c}(x, y)$ das elektrochemische Potential $\mu_{\mathrm{ec}}$ mit der mikroskopischen Stromdichte $\vec{j}_{\text {mikro }}$ verknüpft. Auf einem diskreten Gitter ist diese Art der Beschreibung äquivalent zu einem Netzwerk aus „ohmschen“ Widerständen. Jede Terrassenstufe besitzt dabei eine konstante und isotrope Leitfähigkeit. Entsprechend der Definition einer 1D-Defektlinie wird die gemessene Realstruktur approximiert durch Bereiche kleiner grader Defektlinien. Dies geschieht typischerweise mit der verwendeten Rasterauflösung. Die Terrassenstufe ist eingebettet in das diffuse 2DEG mit seiner spezifischen, zunächst unbekannten Leitfähigkeit $\sigma_{2 \mathrm{DEG}}[\Omega / \square]$. Bei einer Pixelweite von z.B. $1 \AA$ ist die Leitfähigkeit durch eine Terrassenstufe der Länge $l_{\text {Pixel }}$ demnach $G_{\text {Stufe }}^{-1}=\left(\sigma_{\text {Stufe }} \cdot l_{\text {Pixel }}\right)^{-1}+\left(\sigma_{2 D E G}\right)^{-1}$. Die 
Summe ist notwendig, damit bei Abwesenheit einer Stufe $(T \rightarrow 100 \%)$ die Leitfähigkeit an dieser Stelle nicht divergiert und den Wert des 2DEG annimmt. Die Leitfähigkeit des 2DEGs ist $G_{2 D E G}=\sigma_{2 D E G} l_{\text {Pixel }} / l_{\text {Pixel }}=\sigma_{2 D E G}$. Die gemessene Realstruktur kann so in ein Netzwerk diskreter Widerstände bzw. Leitwerte überführt werden. Dieser Modellierung folgend, wurde an jedem Knoten des Netzwerks das Potential mittels STP gemessen. Aufgrund der Rasterung sind die Potentialknoten in einem kubischen Gitter mit je vier Verbindungswiderständen (entsprechend $\mathrm{G}_{\text {Stufe }}$ oder $\mathrm{G}_{2 \mathrm{DEG}}$ ) angeordnet. Die Anforderung bei dieser Art der Modellierung besteht darin, Widerstandsnetzwerke von bis $\mathrm{zu} 10^{6}$ Knoten $\mathrm{zu}$ berechnen. Das verwendete Berechnungsverfahren wird im folgenden Kapitel vorgestellt und im Anschluss daran werden die physikalischen Ergebnisse diskutiert.

\subsubsection{Ein analytisches Verfahren zur Lösung großer Widerstandsnetzwerke}

Betrachtet man ein einfaches Widerstandsnetzwerk bestehend aus Spannungsquellen und Widerständen, so ist das Lösungsschema offensichtlich: Aufgrund der Kontinuitätsgleichung müssen sich die Ströme in allen Knoten, außer an den Quellen und Senken des Stromes (Spannungs- oder Stromquelle), zu null addieren.

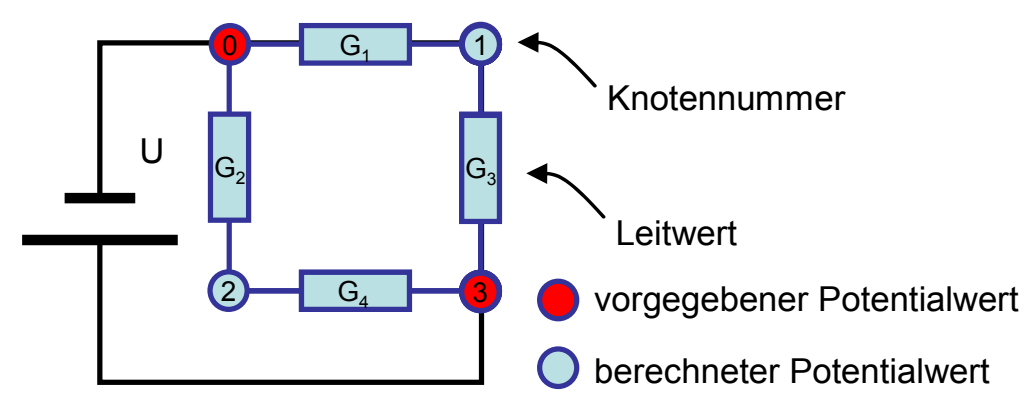

Abbildung 47: Skizze eines einfachen Widerstandsnetzwerks mit vorgegebenen Potentialen an zwei Knoten (rot). Die Knotenpotentiale 1 und 2 sind abhängig von den Leitwerten der eingezeichneten Widerstände.

Abbildung 47 zeigt die Skizze eines einfachen Widerstandnetzwerkes mit einer Spannungsquelle. Die Knoten wurden entsprechend durchnummeriert. Zur Aufstellung eines entkoppelten Gleichungssystems werden die Komponenten des Netzwerks in einzelne logische Elemente zerlegt: 
1. Enthält das Problem k+1 Knoten, so wird zunächst eine leere (k x k)-Matrix aufgestellt. Ein Knoten definiert das Bezugspotential $(U=0)$ und taucht im Weiteren nicht mehr auf. Er wird als Knoten 0 bezeichnet. Die Zeilen und Spalten der Matrix repräsentieren jeweils die nummerierten Knoten. Auf der Diagonalen werden die Summen aller Leitwerte zum jeweiligen Knoten eingetragen. Die Nebendiagonalelemente sind die Leitwerte der Verbindungen zwischen zwei Knoten und werden mit einem negativen Vorzeichen versehen. Für das Widerstandsnetzwerk aus Abbildung 47 bedeutet dies

\begin{tabular}{|c|c|c|c|}
\hline & \#1 & \#2 & \#3 \\
\hline \#1 & $G_{1}+G_{3}$ & 0 & $-G_{3}$ \\
\hline \#2 & 0 & $G_{2}+G_{4}$ & $-G_{4}$ \\
\hline \#3 & $-G_{3}$ & $-G_{4}$ & $G_{3}+G_{4}$ \\
\hline
\end{tabular}

2. Diese Matrix wird durch hinzufügen der Spannungsquellen erweitert. Eine Spannungsquelle zwischen den Punkten $\mathrm{p}$ und q wird allgemein beschrieben durch das Gleichungssystem:

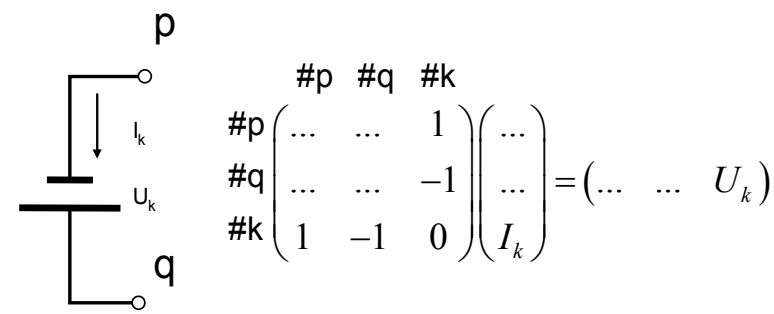

Im angegeben Beispiel setzt die Spannungsquelle nur in Knoten 3 an, da Knoten 0 der Bezugsknoten ist und keinerlei Beachtung bedarf. Es ist leicht zu zeigen, dass sich das entkoppelte Gleichungssystem schreiben lässt als:

$$
\begin{array}{cccc}
\# 1 & \# 2 & \# 3 & \\
\# 2 & \# 3 \\
\# 2 & \left(\begin{array}{cccc}
G_{1}+G_{3} & 0 & -G_{3} & 0 \\
0 & G_{2}+G_{4} & -G_{4} & 0 \\
-G_{3} & -G_{4} & G_{3}+G_{4} & 1 \\
0 & 0 & 1 & 0
\end{array}\right)\left(\begin{array}{c}
U_{1} \\
U_{2} \\
U_{3} \\
I_{1}
\end{array}\right)=\left(\begin{array}{llll}
0 & 0 & 0 & U
\end{array}\right)
\end{array}
$$

Die unbekannten Knotenpotentiale $\mathrm{U}_{1}-\mathrm{U}_{3}$ sind somit eindeutig bestimmt. In der Literatur ist dieses Verfahren als Knotenpotentialverfahren bekannt [116, 117] und Grundlage vieler kommerzieller Programme (z.B. Spice).

Die Stärke dieses Verfahrens liegt darin, dass sich beliebige Netzwerke jeder Konfiguration numerisch gut behandeln lassen. Zu beachten ist dabei allerdings, dass die Matrixgröße bei 
diesem Verfahren quadratisch mit der Anzahl der Knoten und der Spannungsquellen zunimmt. Die zu berechnenden STP-Daten besitzen meist eine Auflösung von 256 x 256 Messpunkten entsprechend 65536 Knoten. Der Bildrand wird als gemessenes Potential vorgegeben womit sich eine quadratische Matrix mit 66560 x 66560 Einträgen (65536 Knoten und 4 x 256 Potentialwerte des Randes) ergibt. Die Matrix besitzt jedoch nur wenige Nebendiagonalelemente $(\sim 4)$ und lässt sich mit Matlab ${ }^{\circledR}$ bei Systemgrößen bis $10^{6} \times 10^{6}$ entsprechend einer STP-Auflösung von 1024 x 1024 Datenpunkten auf aktuellen Computern (1GB, 3GHz) in etwa 15 Minuten lösen.

Im vorliegenden Fall ist jedoch nur die spezifische Stufenleitfähigkeit (siehe Kapitel 3.2.1) und die gemessene Potentiallandschaft (STP-Messung) mikroskopisch bekannt. Um aus diesen Daten weitere Transporteigenschaften des Systems wie z. B. $\sigma_{2 D E G}$ zu extrahieren, wurde eine iterative Anpassungsroutine verwendet. Abbildung 48 verdeutlicht schematisch die Vorgehensweise.
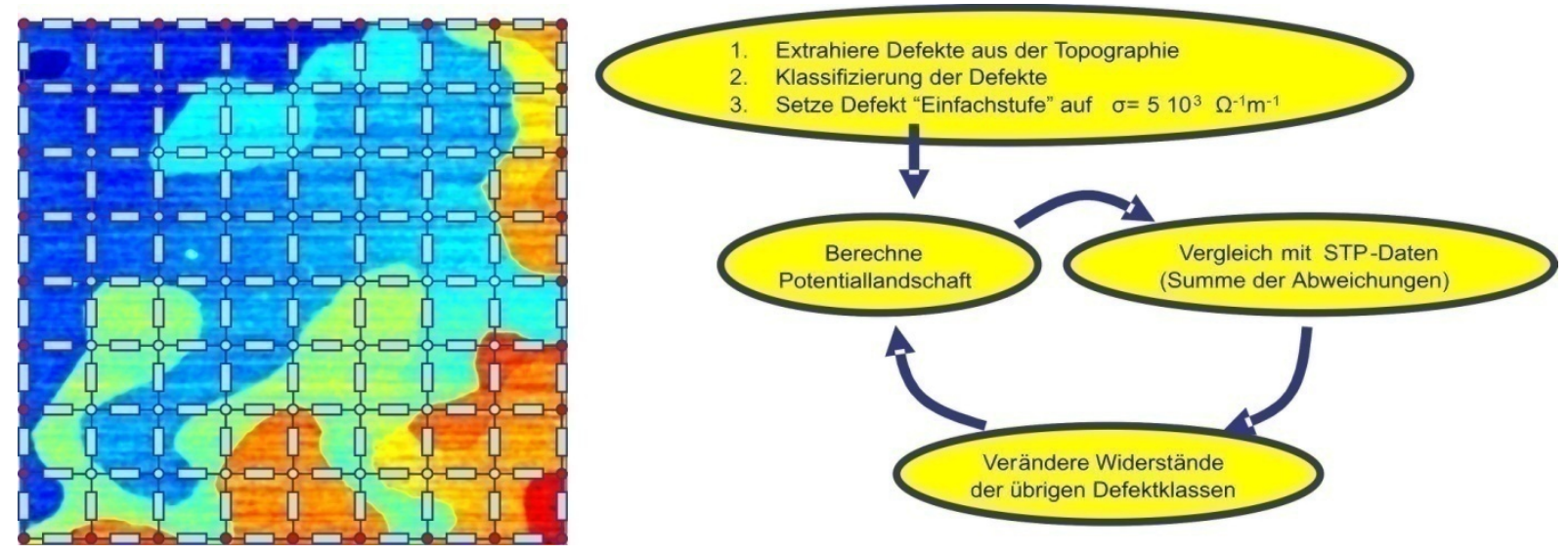

Abbildung 48: Die gemessenen $\mu_{e c, S T P}(x, y)$-Daten werden mit einem kubischen Widerstandsnetzwerk modelliert. Das resultierende Netzwerk verbindet gemessene Potentialknoten mit der vollen Auflösung der STP-Messung (schematisch nicht dargestellt). Das gemessene Potential an den Bildkanten wird als Potentialwert vorgegeben. Die Berechnung der spezifischen Widerstände wird derart ausgeführt, dass zuerst die einzelnen Defekttypen der STMTopographie klassifiziert werden. Einfachstufen wird ein spezifischer Leitwert von $5000 \Omega^{-1} m^{-1}$ zugewiesen. Die Potentiallandschaft wird berechnet und über die Summe der Abweichungsquadrate mit $\mu_{e c, S T P}(x, y)$ verglichen. Die übrigen Defekttypen werden bis zur Konvergenz (typischerweise $\Delta R_{i}<0.1 \Omega$ ) verändert.

Zuerst werden in der STM-Topographie die Terrassenstufen pixelgenau identifiziert. Dies geschieht numerisch mit einer Kantensuchroutine in Matlab ${ }^{\circledR}$. Die vier Verbindungswider- 
stände (Leitwerte) von Potentialknoten, die räumlich auf Terrassenkanten lokalisiert sind, werden auf die Leitfähigkeit $2 G_{\text {Stufe }}$ gesetzt. Den übrigen Verbindungswiderständen (Leitwerten) wird der zunächst noch unbekannte Leitwert $\sigma_{2 \mathrm{DEG}}$ zugewiesen. Das Potential an den Bildkanten wird entsprechend der $\mu_{\mathrm{ec}, \mathrm{STP}}(\mathrm{x}, \mathrm{y})$-Daten gesetzt und fungiert als Randbedingung. Die Potentiallandschaft wird dann mit dem vorgestellten Verfahren berechnet. Die Abweichung zu den Messdaten wird über die Summe der Abweichungsquadrate bestimmt und durch die Variation von $\sigma_{2 \mathrm{DEG}}$ minimiert. Die Optimierungsroutine wurde als "NelderMead-Simplex" Suchmethode implementiert und ist standardmäßig in der Matlab ${ }^{\circledR}$ Programmbibliothek enthalten [118].

Wie bereits in Kapitel 3.2 dargestellt, ist den $\mu_{\mathrm{ec}, \mathrm{STP}}(\mathrm{x}, \mathrm{y})$-Daten häufig ein sehr niederfrequentes Störsignal (Drift) überlagert, was eine Nachbearbeitung in Form von Linien- oder Ebenenabzug notwendig macht. Dies verfremdet jedoch die Potentiallandschaft und beeinflusst die quantitative Anpassung des spezifischen Leitwerts. Um den Einfluss dieser Verfremdung zu minimieren und um eine Aussage zur Signifikanz und Varianz der Ergebnisse machen zu können, werden einige zusätzliche Stützstellen in der Bildmitte verwendet.

Das Potential dieser Knoten wird auf die gemessenen Potentiale der $\mu_{\mathrm{ec}, \mathrm{STP}}(\mathrm{x}, \mathrm{y})$-Daten gesetzt. Für verschiedene Zufallspositionen der zusätzlichen Stützstellen wird der spezifische 2DEGLeitwert ermittelt. Die Häufigkeitsverteilung dieser Werte, wird im Folgenden als Maß für die Signifikanz und Varianz verwendet. Im Anhang A sind hierzu Konsistenzuntersuchungen dargestellt. So wurde beispielsweise der Einfluss zusätzlicher Stützstellen bei normalverteiltem Rauschen der Messdaten untersucht. Ebenso wurde der Einfluss der Stützstellenanzahl bei Ebenen- und Linienabzug auf die Ergebnisse untersucht.

Typische Simulationen werden derart ausgeführt, dass $\sigma_{2 \mathrm{DEG}} \mathrm{Zu}$ einer festen Stützstellenanzahl bei 100 verschiedenen Stützstellenpositionen berechnet wird. Die Stützstellenanzahl wird anschließend variiert um deren Einfluss auf die Ergebnisse analysieren zu können. Zu jeder Konfiguration muss hierbei die Anpassung von $\sigma_{2 \mathrm{DEG}}$ erneut erfolgen, was bei fünf verschiedenen Stützstellenanzahlen mit je 100 zufallsverteilten Positionen einen erheblichen Rechenaufwand von $500 \mathrm{zu}$ berechnende Konfigurationen darstellt.

Das Simulationsprogramm wurde vor diesem Hintergrund so geschrieben, dass es ein verteiltes Rechnen auf beliebig vielen Rechnern unterstützt. Alle zu berechnenden Konfigurationen werden hierfür in einer Arbeitstabelle protokolliert. Jeder Simulationsrechner greift auf diese Tabelle zurück und holt sich die Parameter für seinen Rechenauftrag. Die Ergebnisse werden dann zurück geschrieben. Für die im Folgenden präsentierten Simulationen wurde der 
gesamte CIP-Pool der Physik mit über 60 PCs genutzt. Mit dieser Computeranzahl beträgt die Rechenzeit für die Berechnung eines Datensatzes der Standartauflösung von 256x256 Messpunkten und einem Anpassungswiderstand immerhin noch 24 Stunden bei einer Stützstellenanzahl von [10,20,30,40,50] mit je 100 Konfigurationen und einem freien Anpassungswiderstand. Die Simulationszeit für die Berechnung eines Anpassungswiderstands bei einer Auflösung von 512x512 Messpunkten liegt bei etwa einer Woche.

\subsubsection{Ergebnisse der Modellierung der Transporteigenschaften}

Abbildung 49 (a, b) zeigt $1 \times 1 \mu m^{2}$ der $\operatorname{Si}(111)(\sqrt{ } 3 \times \sqrt{ } 3)$-Ag Oberfläche mit simultan gemessenen $\mu_{\mathrm{ec}, \mathrm{STP}}(\mathrm{x}, \mathrm{y})$-Daten. Die Potentialdaten wurden durch Linienabzug und einen $2 \mathrm{x} 2$ Medianfilter nachbearbeitet. Die numerisch extrahierten Positionen von monoatomaren Terrassenstufen zeigt (c). Das Widerstandsnetzwerk besteht somit aus zwei verschiedenen Leitwerten: Der bereits mikroskopisch bekannte Stufenleitwert $G_{\text {Stufe }}^{-1}=\left(\sigma_{\text {Stufe }} \cdot l_{\text {Pixel }}\right)^{-1}+\left(\sigma_{2 D E G}\right)^{-1}$ und die anzupassenden 2DEG-Leitfähigkeit $G_{2 D E G}=\sigma_{2 D E G} l_{\text {Pixel }} / l_{\text {Pixel }}=\sigma_{2 D E G}$ auf den freien Flächen.

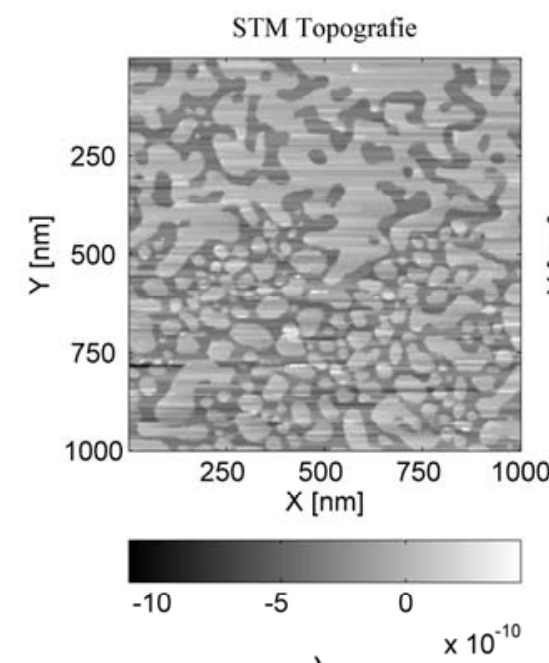

a)
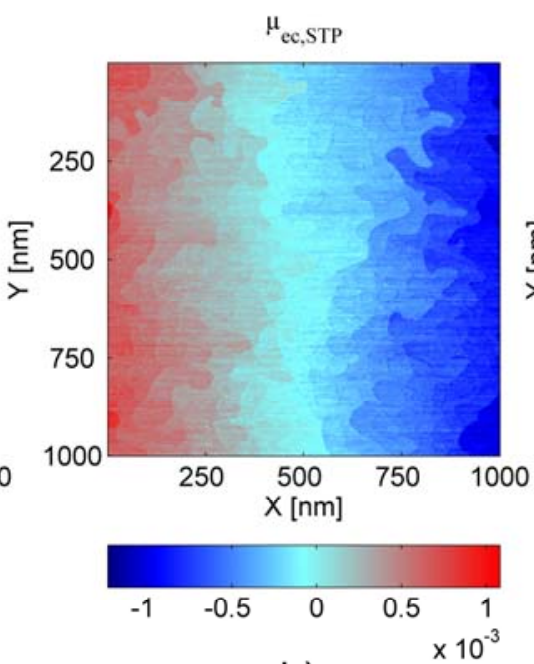

b)

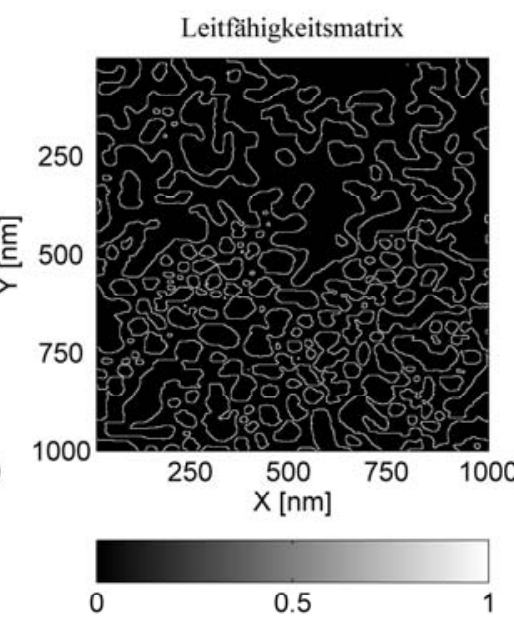

c)

Abbildung 49: STP-Daten von $1 \times x \quad 1 \quad \mu m^{2}$ der $\operatorname{Si}(111)(\sqrt{3} x \sqrt{3})-A g$ Oberfläche bei $j_{\text {extern }}=450 \mathrm{~mA} / \mathrm{m}$. (a) zeigt die Topographie der Oberfläche. (b) illustriert die simultan gemessenen $\mu_{e c, S T P}(x, y)$-Daten und (c) die aus der Topographie gewonnene Leitfähigkeitsmatrix. Die Potentialdaten wurden durch einen Linienabzug und einen $2 x 2$ Medianfilter nachbearbeitet. 
Die nun folgende Berechnung wird mit der vollen Auflösung der experimentellen Messdaten (512x512 Pixel) ausgeführt. Bei der vorliegenden Knotenanzahl von 260000 wurden maximal 50 zusätzliche Stützstellen als Qualitätskontrolle verwendet. Dies entspricht weniger als $0.02 \%$ aller experimentellen Messwerte. Abbildung 50 zeigt die berechnete 2DEGLeitfähigkeit $\sigma_{2 \mathrm{DEG}}$ bei verschieden Stützstellenpositionen und bei Variation der Stützstellenanzahl.

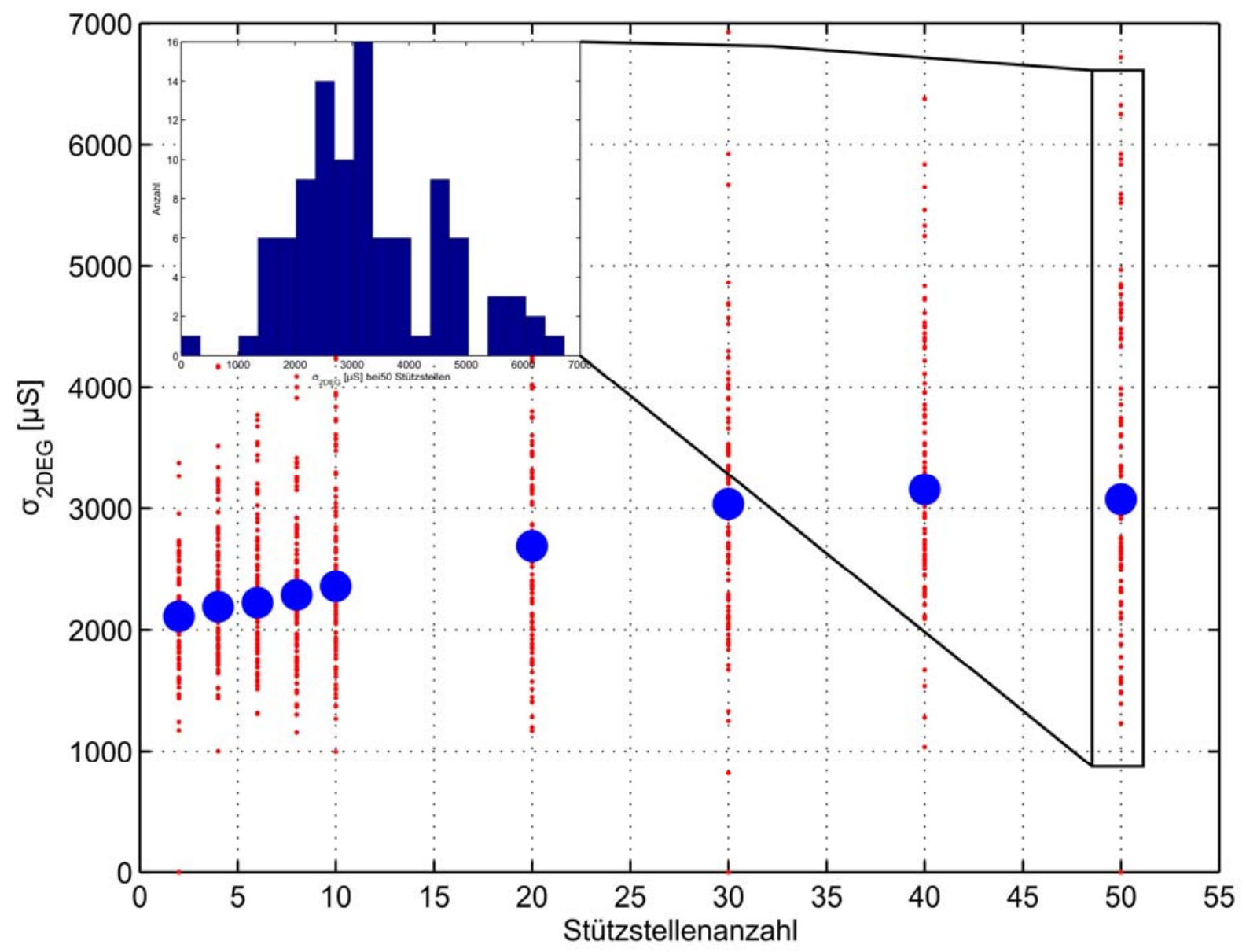

Abbildung 50: Die 2DEG-Leitfähigkeit $\sigma_{2 D E G}$ bei verschiedenen Stützstellenpositionen sowie bei Variation der Stützstellenanzahl. Zu jeder Stützstellenanzahl wurden 100 Konfigurationen mit zufallsverteilten Stützstellen berechnet (rote Punkte). Der Medianwert zu fester Stützstellenanzahl ist als blauer Punkt dargestellt. Bei kleiner Stützstellenanzahl sind systematisch niedrigere Werte zu finden. Oberhalb von 20 Stützstellen bleibt $\sigma_{2 D E G}$ fast konstant. Bei 50 Stützstellen beträgt der Medianwert $\sigma_{2 D E G}=3100 \mu \mathrm{S} / \square$. Der Ausschnitt zeigt hierzu die entsprechende Streuung und die Standardabweichung beträgt $1300 \mu$ S/ $\square$. 
Das Verhältnis nimmt mit der Stützstellenanzahl zu und bleibt oberhalb von 20 Stützstellen nahezu konstant. Im „Anhang A: Konsistenzuntersuchungen zum Einfluss zusätzlicher Stützstellen“ wird dargestellt, dass dieses Verhalten zu erwarten ist, wenn die Potentiallandschaft durch Linien- oder Ebenenabzug verfremdet worden ist. Fasst man die Werte bei $30 \ldots 50$ Stützstellen zusammen, so ergibt sich die 2DEG-Leitfähigkeit zu $\sigma_{2 D E G}=3080 \pm 1100 \mu \mathrm{S} / \square$, bestimmt als Medianwert mit der entsprechenden Standardabweichung. Abbildung 51 zeigt einen Vergleich der gemessenen und errechneten Potentiallandschaften, sowie das Differenzbild mit den Leitfähigkeiten $\sigma_{2 D E G}=3080 \mu \mathrm{S} / \square$ und $\sigma_{\text {Stufe }}=5000 \Omega^{-1} m^{-1}$.
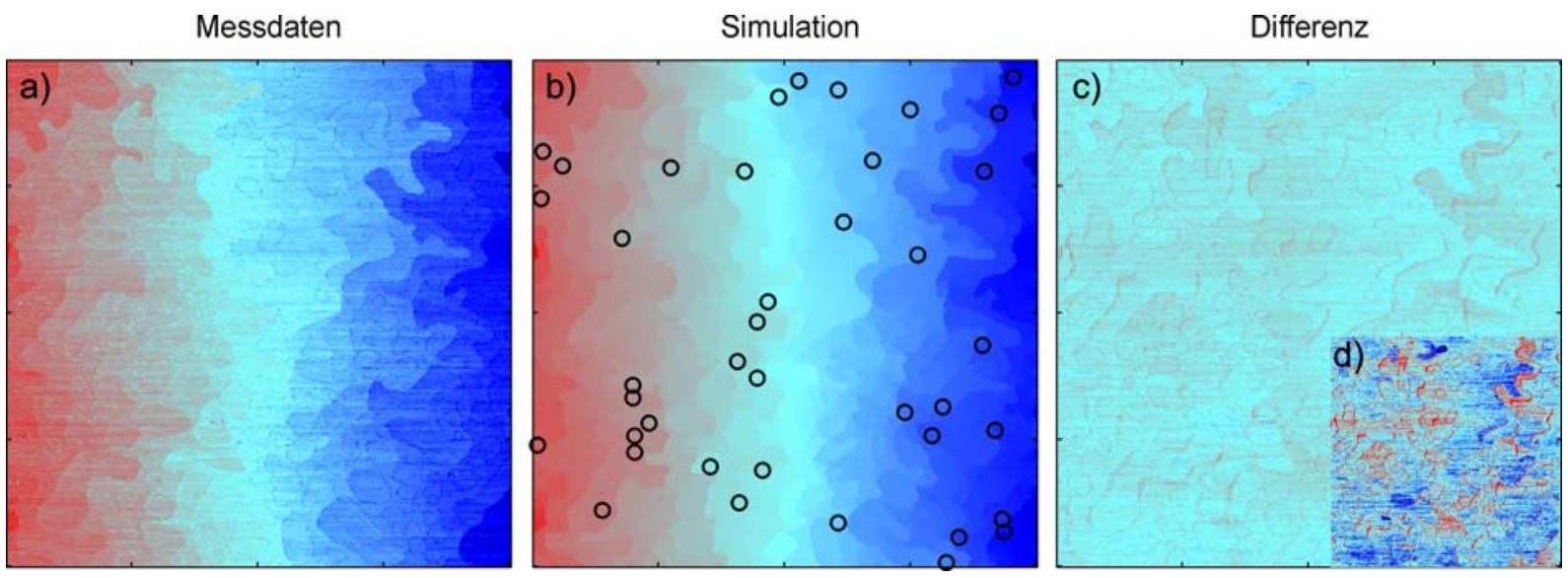

Abbildung 51: Ergebnisse der diffusen Modellierung $\left(\begin{array}{llll}1 & x & 1 & \mu m\end{array}\right)$ : (a) zeigt die gemessenen $\mu_{e c, S T P}(x, y)$-Daten und (b) das Ergebnis der Simulation mit den Leitfähigkeiten $\sigma_{2 D E G}=3080 \mu \mathrm{S} / \square$ und $\sigma_{\text {Stufe }}=5000 \Omega^{-1} m^{-1}$. Die zusätzlichen Stützstellen (40) sind als Kreise eingezeichnet. (c) illustriert das Differenzbild zwischen Messung und Berechnung. Die Datensätze (a-c) sind gleich skaliert und ihr Farbverlauf entspricht von rot nach blau $2.5 \mathrm{mV}$. (d) zeigt das Differenzbild bei einem angepassten Farbverlauf von $600 \mu \mathrm{V}$.

Die berechnete Potentiallandschaft gibt die gemessenen $\mu_{\mathrm{ec}, \mathrm{STP}}(\mathrm{x}, \mathrm{y})$-Daten sehr gut wieder. Der Mittelwert der Absolutdifferenzen ist $54 \mu \mathrm{V}$. Bezogen auf die abgebildete Dynamik von 2,5 mV entspricht dies einer mittleren Abweichung von $2 \%$. Im Differenzbild erkennt man linienartige Störungen und ein Thermospannungssignal an den Stufenkanten. Hiervon abgesehen lassen sich eng begrenzte Bereiche finden, in denen die Simulationen eindeutig von den Messdaten abweichen. Abbildung 52 zeigt den Ausschnitt eines solchen Bereichs. 

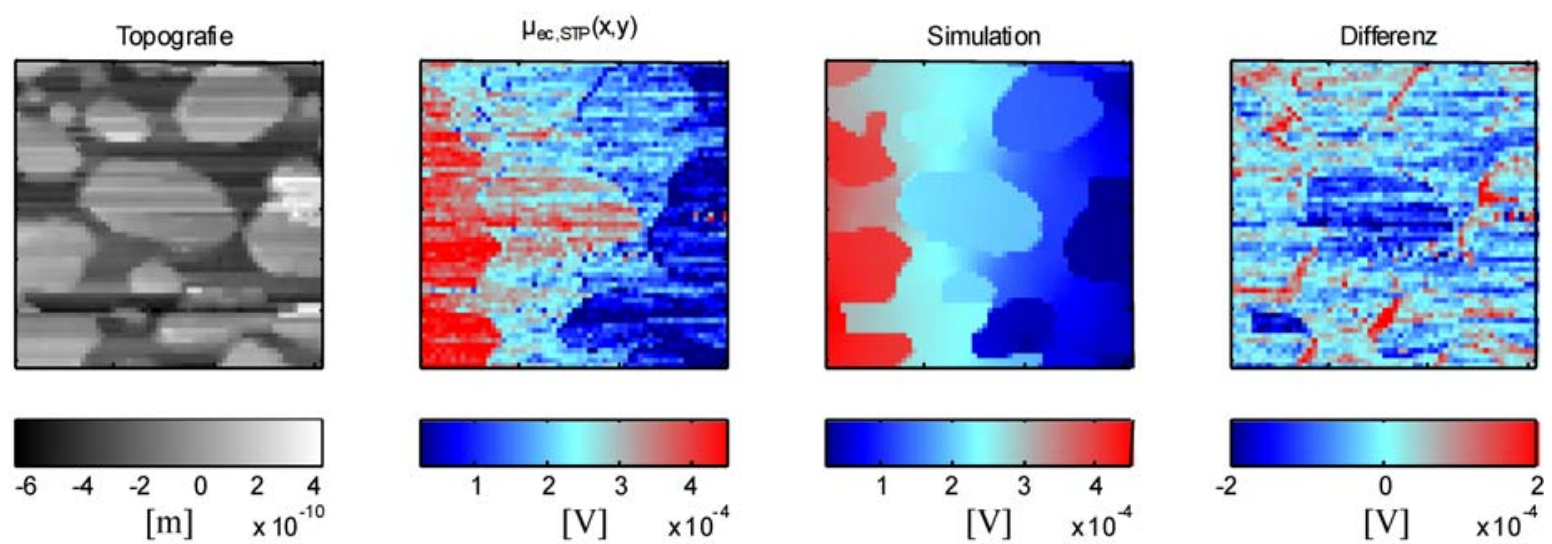

Abbildung 52: Ausschnitt (120 × $\left.120 \mathrm{~nm}^{2}\right)$ eines Bereichs bei der die Simulation von den Messdaten abweicht.

Die große Insel in der Bildmitte liegt bei den Messdaten auf einem Potential von $350 \mu \mathrm{V}$, wohingegen sie in der Simulation ein Potential von $250 \mu \mathrm{V}$ hat. Diese Abweichung von $100 \mu \mathrm{V}$ findet sich dementsprechend im Differenzbild. Die Frage ist, ob die Simulationswerte an dieser Stelle unzutreffend oder aber die Messdaten störbehaftet sind. Beim genauen Analysieren der betreffenden Stelle in den $\mu_{\mathrm{ec}, \mathrm{STP}}(\mathrm{x}, \mathrm{y})$-Daten bemerkt man, dass das Potential von links nach rechts entlang der schnellen Rasterrichtung nachgezogen erscheint. Dem eigentlich tieferen Potential der Insel sind somit Streifen des höheren Potentials aus dem linken Bereich überlagert. Im Streifenmuster der Rasterbewegungen erkennt man immer wieder ungestörte Streifen, dessen Potential dem der Simulation gleicht. Eine weitere Abweichung ist im unteren linken Bereich des Ausschnitts zu erkennen. Aus der Topographie ist ersichtlich, dass sich hier ebenfalls die Spitze modifiziert hat. Da aus den strukturellen Daten die Leitfähigkeitsmatrix extrahiert wird, ist sie an dieser Stelle ebenfalls fehlerhaft was auch diese Abweichungen erklärt.

Abbildung 53 zeigt exemplarisch einen weiteren Bereich, bei der die Simulation systematisch von den $\mu_{\mathrm{ec}, \mathrm{StP}}(\mathrm{x}, \mathrm{y})$-Daten abweicht. Das Potential der sichelförmigen Struktur in der Bildmitte erscheint in der Simulation $140 \mu \mathrm{V}$ zu tief. Rechts daneben findet sich eine Struktur, deren Potential um $150 \mu \mathrm{V}$ zu hoch wiedergegeben wird. 

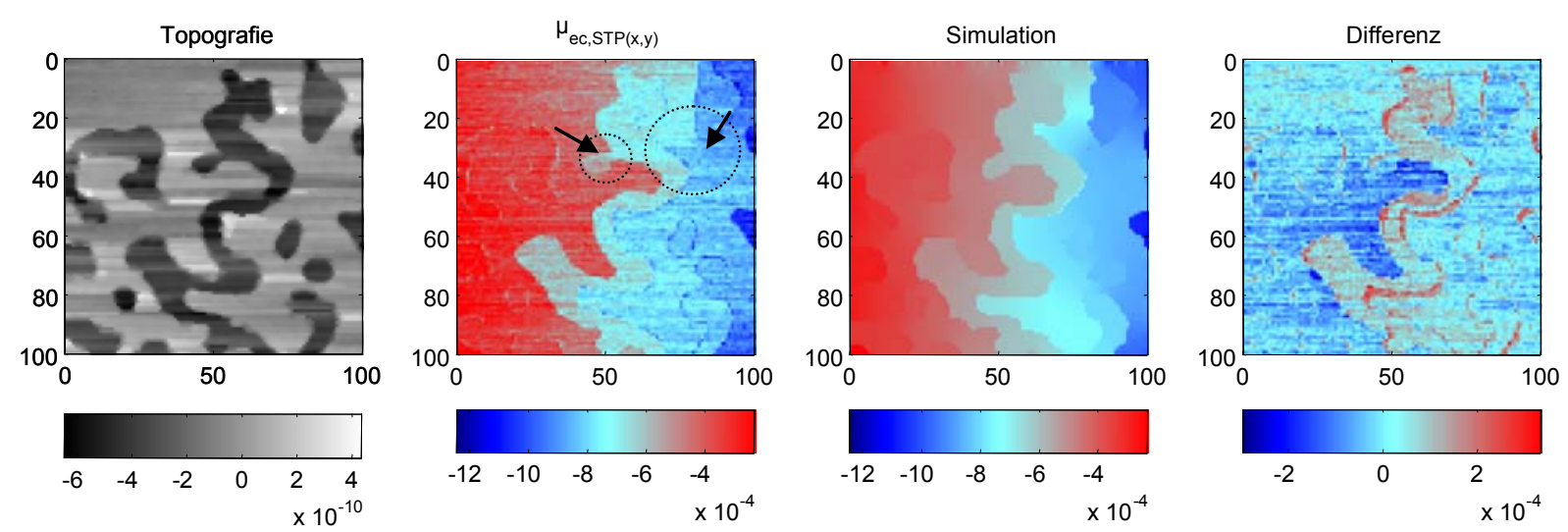

Abbildung 53: Bildausschnitt mit systematischen Abweichungen zwischen gemessenem $\mu_{e c, S T P}$ und Simulation. Die Kreise markieren mögliche Domänengrenzen, welche in der Simulation nicht berücksichtigt wurden. Die Ausrichtung der Domänengrenzen ist durch Pfeile gekennzeichnet.

Zwei Gründe werden für diese Abweichung vermutet. Zum Einen ist bei dieser Bildgröße die STP-Auflösung nicht ausreichend, um Domänengrenzen wie sie in Kapitel 2.1.1 diskutiert worden sind, zu identifizieren. Aufgrund des abrupten Sprungs in $\mu_{\mathrm{ec}, \mathrm{STP}}$ könnten beispielsweise in den markierten Bereichen Domänengrenzen liegen. Insbesondere könnte eine kleine Domänengrenze die betreffende Struktur vom höheren Potential des linken Bereichs entkoppeln. Ein weiterer vielleicht noch wichtigerer Grund ist die Nähe der Struktur zum Rand. Es ist auffällig, dass der obere Rand der Struktur linienartige Störungen mit erhöhtem Potential zeigt. Dieses, durch Störungen erhöhte Potential einiger weniger Rasterlinien, bestimmt dann als Randwert das Potential der Struktur. Die Struktur bekommt folglich ein insgesamt überhöhtes Potential. Mit diesen Begründungen lassen sich nahezu alle Differenzen zwischen den Messdaten und der Simulation auf Artefakte des Messprozesses zurückführen.

Aufgrund der kleinen mittleren Abweichungen von 2\%, welche fast vollständig auf Artefakte des Messprozess zurückgeführt werden können, erscheint die diffuse Modellierung geeignet, die mikroskopischen Transporteigenschaften der $\operatorname{Si}(111)(\sqrt{3} \mathrm{x} \sqrt{3})-\mathrm{Ag}$ Oberfläche zu modellieren. Obwohl die langsame Rasterrichtung durch Driftkorrekturen in Form von Ebenen- und Linienabzug verfremdet worden ist, konnten aus der nachbearbeiteten Potentiallandschaft gemäß Anhang A dennoch die mikroskopischen Transporteigenschaften quantifiziert werden. Die Leitfähigkeit des ungestörten 2DEG ergibt sich demnach zu $\sigma_{2 D E G}=3080 \mu \mathrm{S} / \square$ und die Leitfähigkeit über monoatomare Stufen zu $\sigma_{\text {Stufe }}=5000 \Omega^{-1} m^{-1}$. 
Auf der Grundlage dieser Modellierung können nicht nur die mikroskopischen Transporteigenschaften des Systems bestimmt werden. Zusammen mit der Realstruktur können ebenfalls die lokalen Stromdichten durch das Perkolationsnetzwerk berechnet werden. Abbildung 54 (c) zeigt die räumliche Stromdichte für den bereits diskutierten Datensatz. Die Stromdichte ist stark inhomogen und schwankt zwischen (0-5) A/m. Ein ausgezeichneter Perkolationspfad mit einer mittleren Stromdichte von ca. $3 \mathrm{~A} / \mathrm{m}$ ist in der oberen Bildhälfte zu erkennen. In der unteren Bildhälfte besteht das Perkolationsnetzwerk aus vielen kleineren Inseln, die vom Strom umflossen werden. (d) zeigt die zweidimensionalen Stromdichte aus diesem Bereich als Vektorplot. Die Stromdichte teilt sich in eine rechts- und linksherum fließende Komponente auf, um sich nach der Insel wieder zu vereinigen. ${ }^{21}$

Diese Ergebnisse zeigen eindrucksvoll, dass der elektrische Transport vor allem entlang favorisierter Perkolationspfade erfolgt. Die makroskopisch vermessene Leitfähigkeit durch die $\operatorname{Si}(111)(\sqrt{3} \times \sqrt{3})$-Ag Rekonstruktion ist daher neben den mikroskopischen Transporteigenschaften auch von den strukturellen Eigenschaften, die das Perkolationsnetzwerk beschreiben, abhängig. Diesem Sachverhalt widmet sich das nächste Kapitel.

\footnotetext{
${ }^{21}$ Interessante Interferenzeffekte werden an solchen Strukturen bei tiefen Temperaturen vermutet. In der Zukunft wird die experimentelle Möglichkeit bestehen, Studien bei bis zu 12 T und 5.6 K durchführen zu können. Prinzipiell könnte damit $\mu_{\mathrm{ec}}(\mathrm{x}, \mathrm{y})$ in Aharonov-Bohm Strukturen mit räumlicher Auflösung untersucht werden.
} 

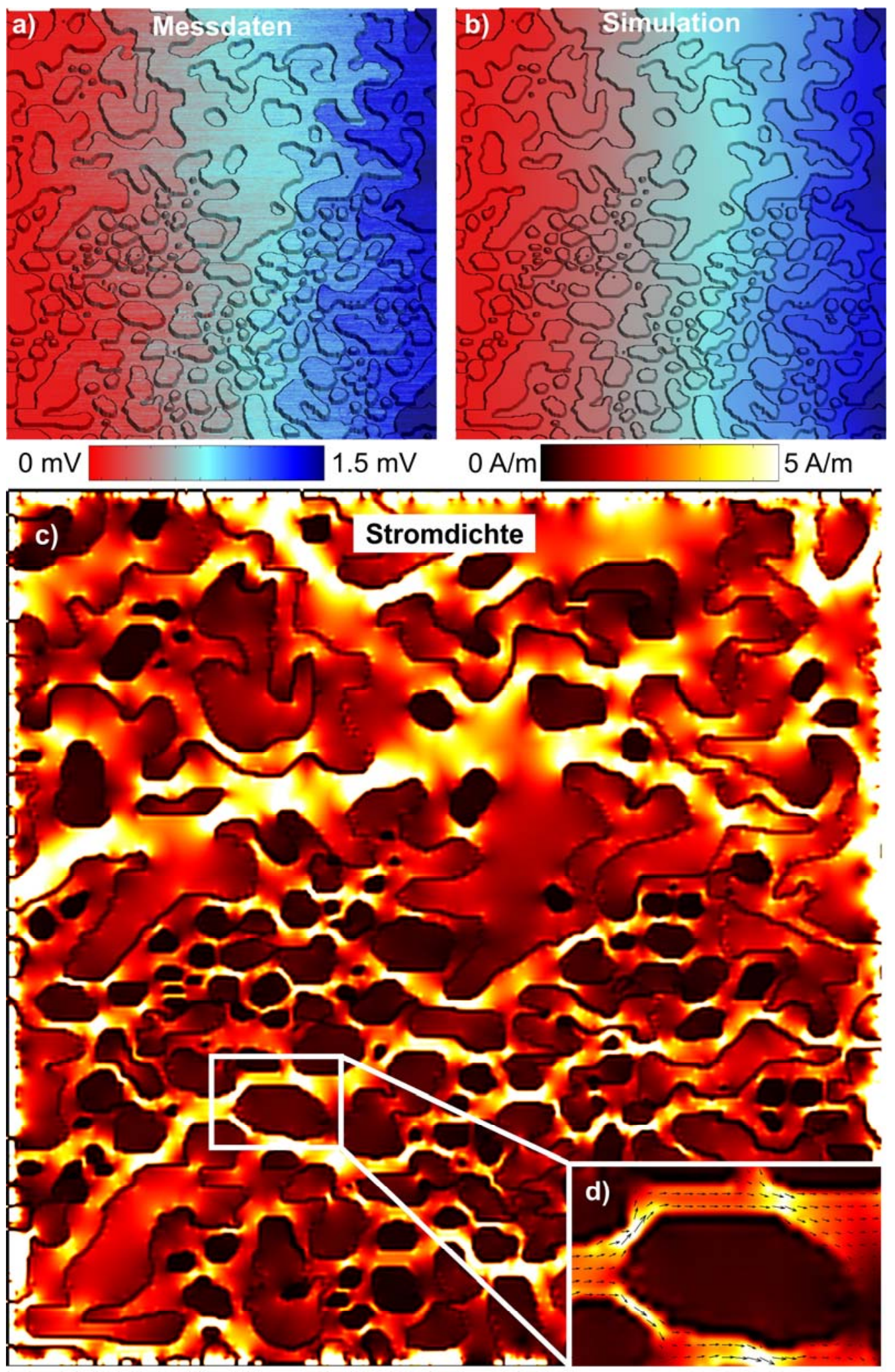

\section{Stromdichte}




\subsubsection{Von den mikroskopischen Transporteigenschaften zur makroskopi- schen Leitfähigkeit}

Im vorangegangen Kapitel wurden die Transporteigenschaften der $\operatorname{Si}(111)(\sqrt{3} \times \sqrt{3})-A g$ Oberfläche erstmalig vollständig mikroskopisch charakterisiert. Zusätzlich findet sich in der Literatur eine Vielzahl von Untersuchungen zur makroskopischen Leitfähigkeit dieser Oberfläche. Eine Ankopplung der mikroskopischen Werte an makroskopisch bestimmte Leitfähigkeiten, meist in Form von Vierpunktmessungen, gelang bisher nicht. Im vorliegenden Fall ist jedoch die Realstruktur durch STM-Topographien im Detail bekannt und es kann der Versuch unternommen werden, zusammen mit den mikroskopisch bestimmten Transporteigenschaften, die sich daraus ergebende makroskopische Leitfähigkeit der Probe zu bestimmen. Abbildung 55 (a) zeigt eine STM-Topographie der Oberfläche von 1.5 x $1.5 \mu \mathrm{m}^{2}$.

a)

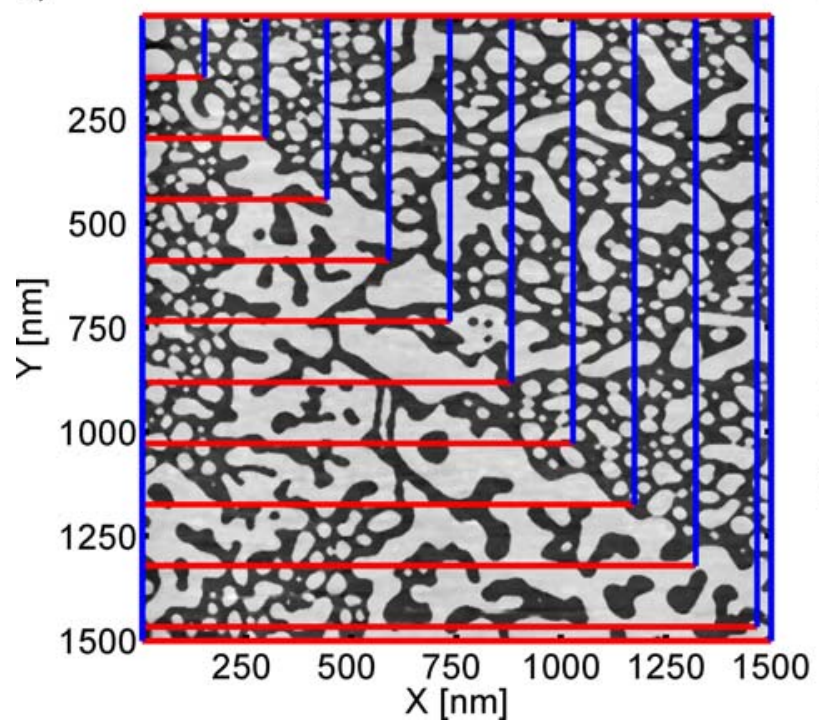

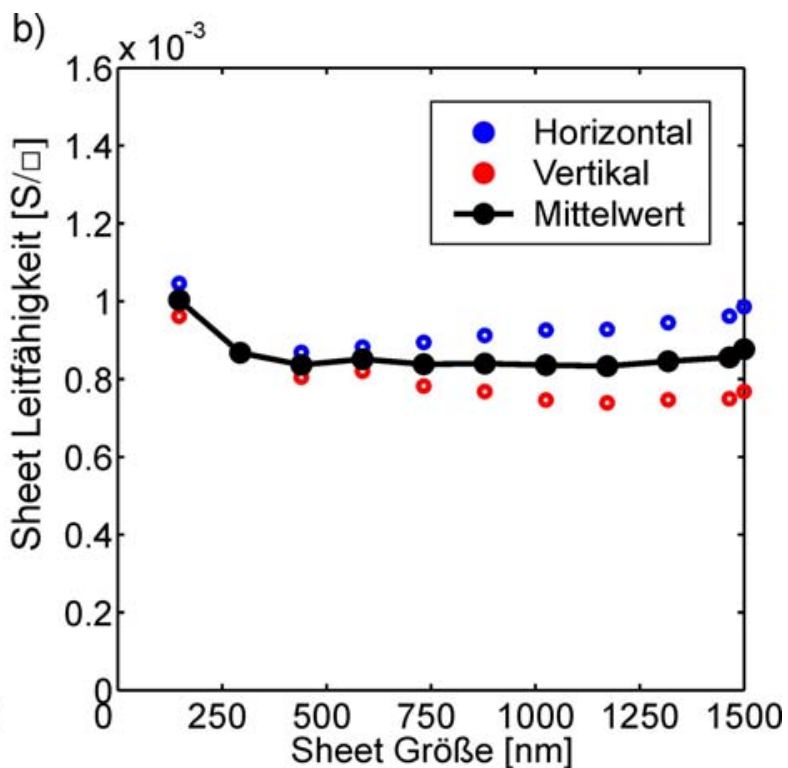

Abbildung 55: (a) Eine große STM-Topographie $\left(1.5 \times 1.5 \mu^{2}\right)$ wurde benutzt um aus den mikroskopischen Leitfähigkeiten $\left(\sigma_{\text {Stufe }}=5000 \Omega^{-1} m^{-1}\right.$ und $\left.\sigma_{2 D E G}=3080 \mu \mathrm{S} / \square\right)$ die makroskopische Leitfähigkeit der Probe zu berechnen. Für verschiedene quadratische Ausschnitte wurde die Leitfähigkeit in horizontaler (blau) und vertikaler (rot) Richtung berechnet. Die farbigen Striche illustrieren die verwendeten Elektroden an zwei gegenüberliegenden Seiten des verwendeten Bereichs. (b) Größenabhängig ergeben sich unterschiedliche Leitfähigkeiten durch das Perkolationsnetzwerk. Oberhalb von $500 \mathrm{~nm}$ Bildgröße bleibt der Mittelwert der Leitfähigkeiten beider Richtungen unabhängig von der Stromrichtung nahezu konstant $(850$ $\mu S / \square)$. 
Die Topographie erscheint zunächst bei diesem Probenort stark inhomogen. Links der Bilddiagonalen ist die größte Strukturgröße auf der oberen Terrasse, wo hingegen sie rechts der Bilddiagonalen auf der unteren Terrasse zu finden ist. Berücksichtigt man jedoch, dass es keinen Unterschied zwischen der unteren und oberen Terrasse gibt, so erscheint dieser Ausschnitt gleichwohl homogener, d.h. mit denselben Strukturgrößen rechts wie links der Bilddiagonalen. Mit dieser Realstruktur wurde berechnet, was eine ideale Vierpunktmessung ergeben würde. Mit den mikroskopisch bestimmten Leitfähigkeiten, d.h. $\sigma_{\text {Stufe }}=5000 \Omega^{-1} \mathrm{~m}^{-1}$ und $\sigma_{2 \mathrm{DEG}}=3080 \mu \mathrm{S} / \square$ wurde die Leitfähigkeit bei verschiedenen quadratischen Bildgrößen berechnet. Ladungstransport konnte nur im jeweiligen Innenraum stattfinden und die Elektroden wurden durch je zwei gegenüberliegende Seiten des Quadrats modelliert. Die berechneten Sheet-Leitfähigkeiten in Abhängigkeit von der Bildgröße zeigt (b). Fast unabhängig von der verwendeten Bildgröße ist die Leitfähigkeit in horizontaler Richtung größer als in der Vertikalen. Für das gesamte Bild beträgt diese Anisotropie ca. $20 \%$. Sie ist direkt verknüpft mit der räumlichen Struktur des Perkolationsnetzwerks. Viele Strukturen sind horizontal elongiert, so dass sie eine erhöhte Barriere für den vertikalen Stromfluss darstellen. Obgleich die Leitfähigkeiten für die horizontale und vertikale Stromrichtung leicht unterschiedlich sind, ist ihr Mittelwert oberhalb von $500 \mathrm{~nm}$ Bildgröße nahezu konstant. Er beträgt ca. $850 \mu \mathrm{S} / \square$.

Die Analyse eines weiteren interessanten Probenorts zeigt Abbildung 56. Eine monoatomare Substratstufe, die wahrscheinlich nicht durch den Wachstumsprozess entstanden ist, sondern bereits vor der Präparation auf dem Si-Wafer vorhanden war, durchkreuzt den gesamten Probenbereich. Interessanterweise scheint die Substratstufe die Perkolationsbildung in ihrer direkten Umgebung zu behindern. Ein ca. $75 \mathrm{~nm}$ breiter Streifen ohne Terrassen ist die Folge. Er ist ein niederohmiger Kanal in der horizontalen Richtung durch das Perkolationsnetzwerk. Für den vertikalen Stromtransport ist die Stufenkante jedoch ein nicht perkoliertes Hindernis. Abbildung 56 (b) zeigt die entsprechende Analyse. Wenn aufgrund der geometrischen Wahl des verwendeten Ausschnitts die Substratstufe bspw. in der horizontalen Richtung zum Tragen kommt, ist ein Anstieg der Leitfähigkeit zu beobachten. Entgegengesetztes gilt für die vertikale Stromrichtung. An diesem Probenort verändern sich die Leitfähigkeiten für beide Stromrichtungen oberhalb von $1 \mathrm{x} 1 \mu \mathrm{m}^{2}$ fast gar nicht mehr. Ähnlich wie zuvor bleibt der Mittelwert oberhalb von $500 \mathrm{~nm}$ Bildgröße bei $820 \mu \mathrm{S} / \square$ konstant. 
a)

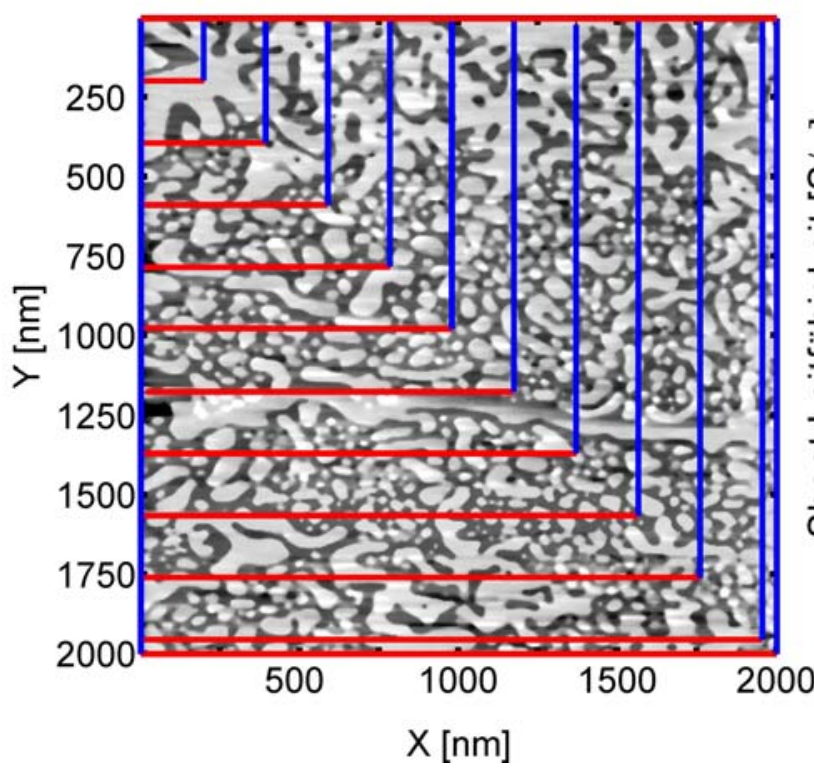

b)

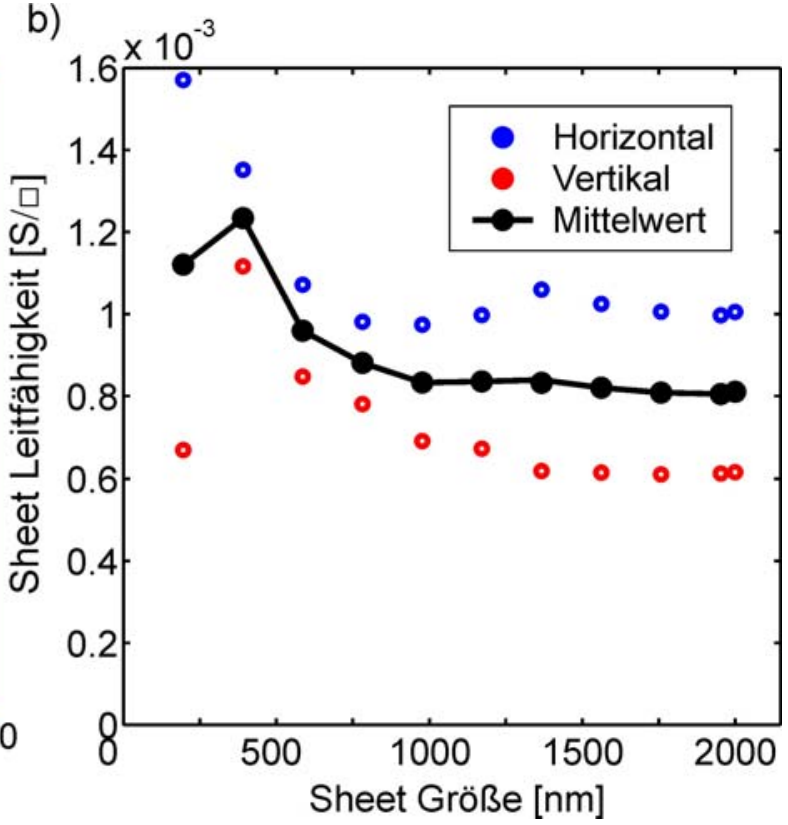

Abbildung 56: Berechnung der makroskopischen Sheet-Leitfähigkeit in einem Probenbereich mit gleichmäßiger Perkolationsstruktur. Für verschiedene Bildgrößen wurde die Leitfähigkeit in horizontaler (blau) und vertikaler (rot) Richtung berechnet. Eine durchgehende monoatomare Stufe des Substrats führt bei einer Bildgröße von $1350 \mathrm{~nm}$ zu einer Erhöhung (horizontal - blau) bzw. Verringerung der Leitfähigkeit. Bei Bildgrößen oberhalb von $500 \mathrm{~nm}$ bleibt die Sheet-Leitfähigkeit konstant. Der Mittelwert beider Stromrichtungen beträgt ab $1 \mu \mathrm{m}^{2}$ Bildgröße ca. $820 \mu \mathrm{S} / \square$.

Diese Ergebnisse lassen sich dahingehend deuten, dass das natürliche Perkolationsnetzwerk der $\operatorname{Si}(111)(\sqrt{3} \mathrm{x} \sqrt{ } 3)$-Ag Rekonstruktion einer gewissen Statistik folgt und oberhalb von $500 \mathrm{~nm}$ quadratischer Bildgröße ein sinnvoller Mittelungsprozess über verschiedene Perkolationspfade einsetzt. Bis zur maximal untersuchten Bildgröße von 2 × $2 \mu \mathrm{m}^{2}$ bleibt der Mittelwert aus horizontaler und vertikaler Leitfähigkeit bei $\sim 800 \mu \mathrm{S} / \square$ konstant.

Es ist naheliegend, diese mikroskopisch bestimmte Leitfähigkeit durch die $\operatorname{Si}(111)(\sqrt{3} x \sqrt{3})-\mathrm{Ag}$ Rekonstruktion mit der Leitfähigkeit, wie sie makroskopisch auf dieser Probe bestimmt worden ist, zu vergleichen. In Kapitel 2.3.1 wurde die Leitfähigkeit durch die Rekonstruktion $\mathrm{zu}(460 \ldots 520) \mu \mathrm{S} / \square$ auf Grundlage des Gesamtwiderstands zwischen den Kontakten abgeschätzt. Im Vergleich zu $800 \mu \mathrm{S} / \square$ aus der mikroskopischen Bestimmung ist dies eine erstaunliche Übereinstimmung, betrachtet man erstens die geringe Größe des analysierten Bereichs von $4 \mu \mathrm{m}^{2}$ im Vergleich zur gesamten Probe von $12 \cdot 10^{6} \mu \mathrm{m}^{2}$ und zweitens die Ungenauigkeiten bei der Bestimmung der Leitfähigkeit durch die Rekonstruktion aus dem Gesamtwiderstand in einer Zweipol-Messung (siehe Kapitel 2.3.1). 
Dies eröffnet die Möglichkeit, die gemachten Analysen, welche ausschließlich auf mikroskopischen Messungen beruhen, mit makroskopischen Vierpunktmessungen anderer Arbeitsgruppen zu vergleichen. Abbildung 57 zeigt eine Zusammenfassung von Literaturwerten mit ihren Elektrodenabständen. Mit kleiner werdenden Elektrodenabständen wurden immer höhere Leitfähigkeiten gemessen. Bei einem Elektrodenabstand von nur noch wenigen Mikrometern wird die Leitfähigkeit durch diese Rekonstruktion zu $\sim 800 \mu \mathrm{S} / \square$ spezifiziert. Dies ist exakt der Wert gegen den die Leitfähigkeit durch das Perkolationsnetzwerk auf Basis der mikroskopischen Transporteigenschaften konvergiert.

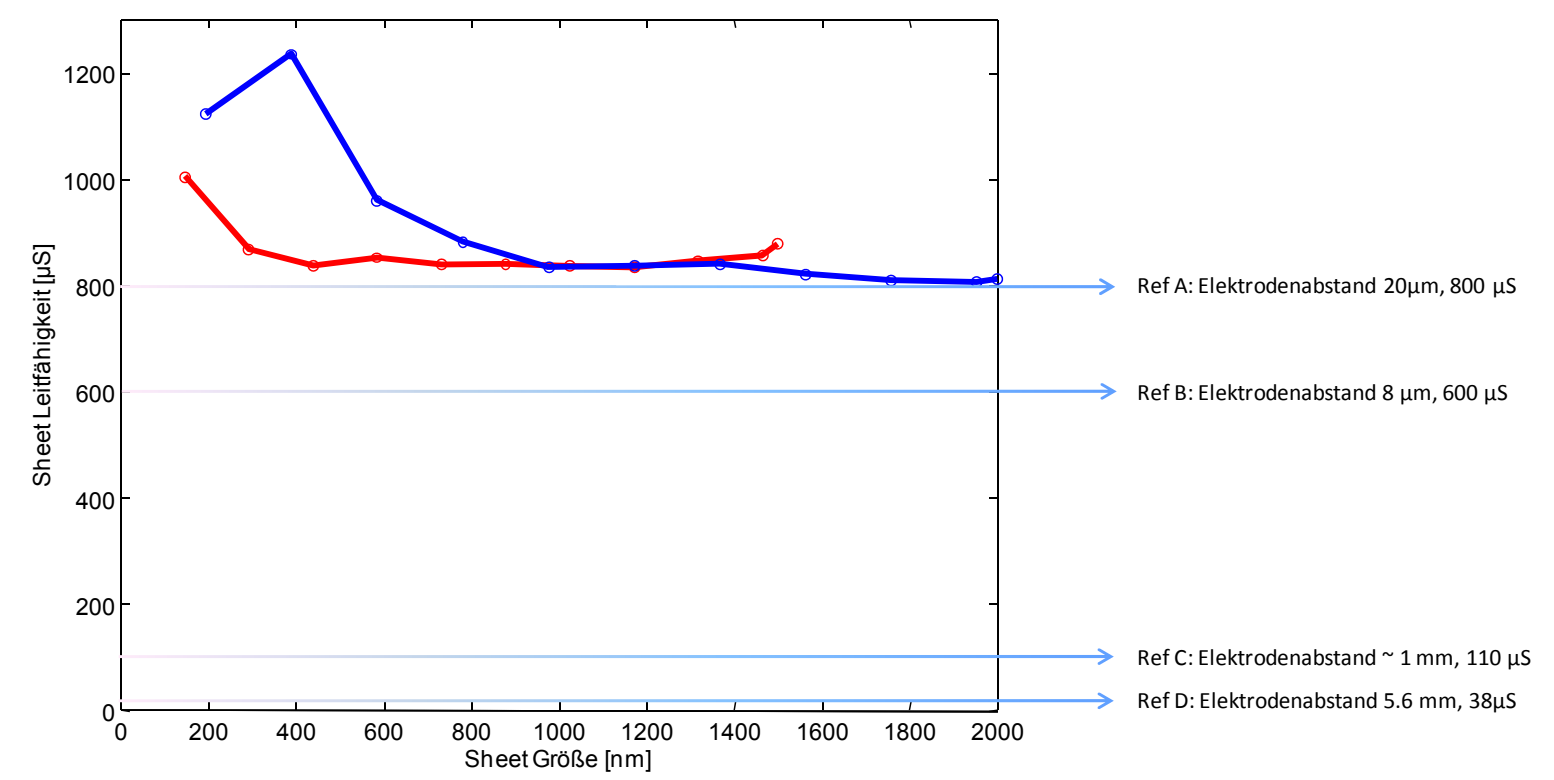

Abbildung 57: Darstellung der berechneten Sheet-Leitfähigkeit auf Grundlage der mikroskopisch bestimmten Transporteigenschaften (rot + blau) zusammen mit Literaturangaben. Alle Literaturangaben sind Vierpunktmessungen. Mit kleiner werdendem Elektrodenabstand werden größere Leitfähigkeiten gefunden. Bei einem Elektrodenabstand von nur noch $20 \mu \mathrm{m}$ werden $800 \mu \mathrm{S}$ spezifiziert. Dies ist der Wert gegen den die errechnete SheetLeitfähigkeit unter Verwendung mikroskopischer Größen konvergiert. Referenzen: A,B:[106], $C:[78] D:[63]$

Bei einem Elektrodenabstand von nur $1 \mu \mathrm{m}$ wurde im Jahr 2003 die Leitfähigkeit zu 1500 $\mu \mathrm{S} / \square$ spezifiziert [107]. Dieser hohe Wert ist im Rahmen der hier gezeigten Ergebnisse dadurch zu erklären, dass ein fast atomar glatter, gut perkolierter Bereich vermessen worden ist. Nach Abbildung 56 könnte der Probenort bspw. parallel und in der Nähe einer Substrat- 
stufe gelegen haben. ${ }^{22}$ Die große Streuung der Literaturwerte kann somit wie folgt erklärt werden: Die gemessenen Leitfähigkeiten sind Leitfähigkeiten durch das Perkolationsnetzwerk und nicht intrinsische Eigenschaften des 2DEGs. Bei großen Elektrodenabständen wird das Perkolationsnetzwerk inklusive weiterer Defekte (Substratstufen, „Stepbunching“ etc.) vermessen. Geringe Leitfähigkeiten sind die Folge. Da diese Effekte mit verringertem Elektrodenabstand minimiert werden, wurden ansteigende Leitfähigkeiten in jüngeren Publikationen beobachtet.

${ }^{22}$ Auf einen Vergleich mit Ergebnissen von Ref. [108] (3000 $\left.\mu \mathrm{S} / \square\right)$ wird an dieser Stelle verzichtet. Die Autoren haben p-dotiertes Silizium verwendet. Eine Akkumulationszone unter dem 2DEG kann daher nicht ausgeschlossen werden (siehe hierzu bspw. Abbildung 34). 


\subsection{Raster-Tunnel-Potentiometrie auf der $\operatorname{Si}(111)(\sqrt{3} \times \sqrt{3})-A g$ Rekonstruktion auf stark gestuften Silizium Substraten}

Im Verlauf dieser Dissertation entstand eine enge Zusammenarbeit mit der Arbeitsgruppe von Prof. R. Möller von der Universität Duisburg-Essen, welche ebenfalls mittels Raster-TunnelPotentiometrie den Ladungstransport durch $\operatorname{Si}(111)(\sqrt{3} \mathrm{x} \sqrt{3})$-Ag Rekonstruktion untersucht. ${ }^{23}$ Im Unterschied zu den bisher vorgestellten Ergebnissen auf unverkippten Substraten wurden in Duisburg vizinale Si-Substrate verwendet. Die in Abschnitt 2.3.4 beschriebene Perkolationsbildung ist hierbei unterbunden, so dass es zur Ausbildung einer gestuften Oberfläche mit atomar glatten Terrassen kommt. Während das natürliche Perkolationsnetzwerk auf unverkippten Substraten eine isotrope Unordnung aufweist, ist das 2DEG auf verkippten Substraten durch zueinander parallele und ausgedehnte Stufen gestört. Ein Beispiel dieser Oberfläche zeigt Abbildung 58 (a). In Anlehnung an Vierpunktmessungen der Arbeitsgruppe Hasegawa, wurde der Ladungstransport durch diese Strukturen in Abhängigkeit von der externen Stromrichtung parallel und senkrecht zur präferierten Stufenrichtung untersucht. Ergebnisse der Duisburger Arbeitsgruppe für beide Stromrichtungen zeigt Abbildung 58 (b, e). Eine detaillierte Beschreibung der experimentellen Daten ist in der Diplomarbeit von Evgeny Zubkov, sowie der Dissertation von Amin Bananni zu finden [22, 119]. Die im Rahmen dieser Arbeit entwickelten Analysemethoden wurden ebenfalls verwendet, um die mikroskopischen Transportprozesse dieses Systems zu identifizieren. Eine Analyse wurde auf Basis der in Kapitel 3.2.2 vorgestellten Methoden durchgeführt und soll im Folgenden kurz vorgestellt werden.

Die für die Messungen verwendeten Substrate unterscheiden sich von den Volumeneigenschaften her nicht von den in dieser Arbeit verwendeten Proben (Si:P, $130 \Omega \mathrm{cm})$. Der einzige Unterschied $\mathrm{zu}$ den bereits vorgestellten Messungen besteht darin, dass Wafer mit $0.5^{\circ}$ Fehlneigung von der (111)-Richtung verwendet worden sind, was zu vizinalen Oberflächen führt. Die $\operatorname{Si}(111)(\sqrt{ } 3 x \sqrt{ } 3)$-Ag Rekonstruktion wurde gemäß Abschnitt 2.2 präpariert. Die Oberfläche dieser Proben besteht aus atomar glatten Terrassen, die durch Si-Stufen (monoatomare, doppel und dreifach Stufen) mit fast paralleler Ausrichtung und einer Stufendichte von ca. $30 \mathrm{Stufen} / \mu \mathrm{m}$ getrennt sind. Ein externer Querstrom wurde durch zwei

\footnotetext{
${ }^{23}$ Arbeitsgruppe Prof. Möller, Fachbereich Physik der Universität Duisburg-Essen, 47048 Duisburg
} 
freibewegliche Goldspitzen im Abstand von (50..300) $\mu \mathrm{m}$ aufgeprägt. Sowohl die Kontaktierung durch die Goldspitzen, als auch die Positionierung der Tunnelspitze konnte durch ein Raster-Elektronen-Mikroskop mit einer lateralen Auflösung von ca. $100 \mathrm{~nm}$ überwacht werden. Dieser experimentelle Aufbau ermöglicht die Analyse des Ladungstransports durch eine gegebene Struktur bei in der Ebene rotierender Stromrichtung.

Abbildung 58 zeigt auf diese Weise durchgeführte STP-Messungen. Bei den Messdaten (a, b) ist die extern aufgeprägte Stromrichtung senkrecht zur präferierten Stufenrichtungen. Auf den freien Terrassenflächen lässt sich ein Gradient auflösungsbedingt nur sehr schwach erkennen und die Potentialsprünge spiegeln maßgeblich die Terrassenstruktur wieder.

Abbildung 58 (d, e) zeigt STP-Daten für die Querstromrichtung parallel zur präferierten Stufenrichtung. Es lässt sich deutlich erkennen, dass in diesem Fall der Stromfluss auf enge Kanäle, welche durch die Terrassenstruktur geformt werden, eingeengt ist. 

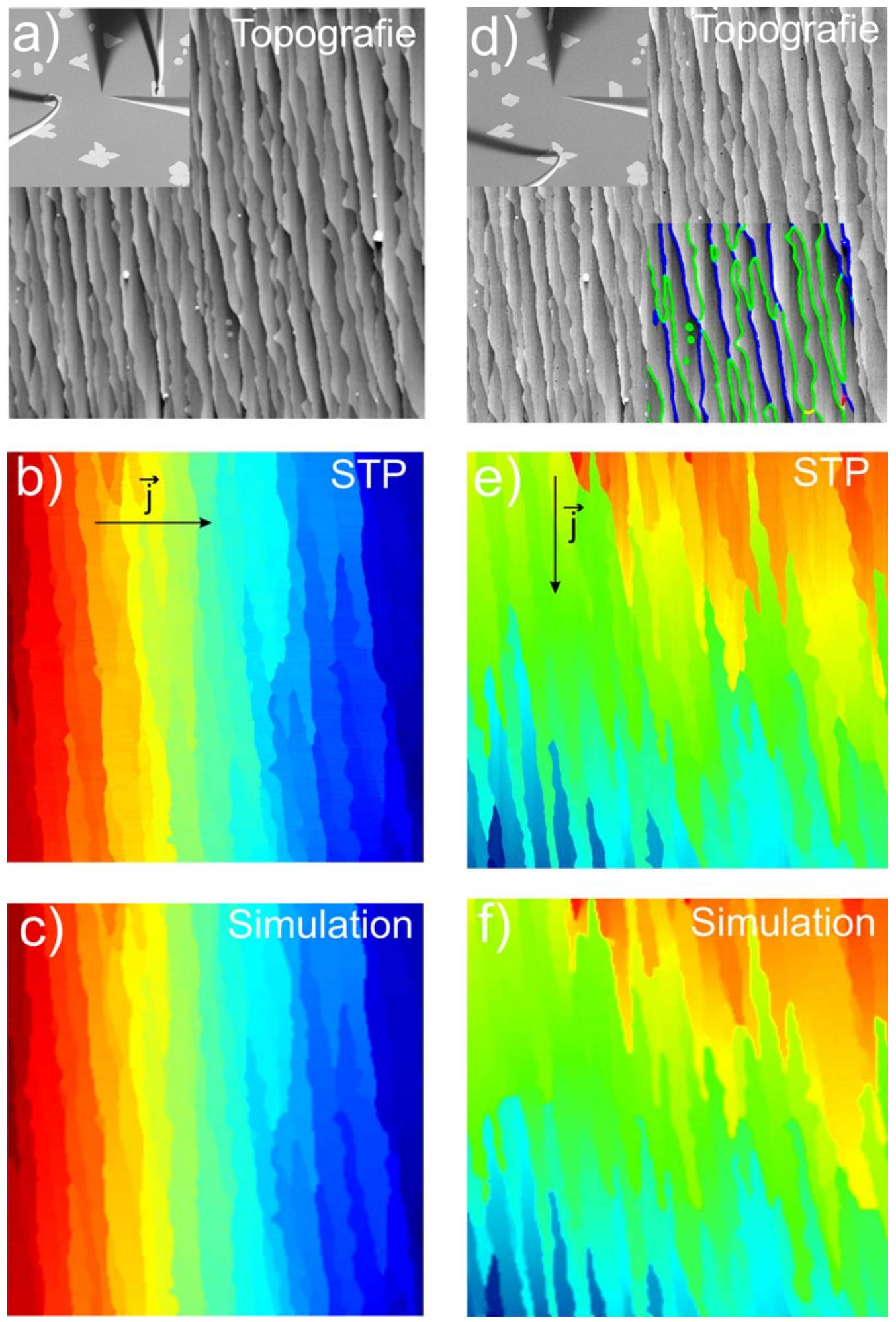

Abbildung 58: STP-Daten für parallele und senkrechte externe Querstromrichtungen zur präferierten Stufenrichtung. Die Stromdichte beträgt in (a, b) $0.4 \mathrm{~A} / \mathrm{m}$ und in (d, e) $0.26 \mathrm{~A} / \mathrm{m}$. Der Bildausschnitt in (d) zeigt die Defekt-Klassifizierung (grün: monoatomare Stufen, blau: mehrfach Stufen, gelb: Domänengrenze A, rot: Domänengrenze B) und (c, f) zeigen die entsprechenden Simulationen. 
Der Ladungstransport durch diese Strukturen wurde mit dem in Abschnitt 3.2.2 beschrieben Verfahren untersucht. Um die STP-Daten erfolgreich beschreiben zu können, mussten zusätzlich zur 2DEG-Leitfähigkeit auch spezifische Leitfähigkeiten für Mehrfachstufen und zwei unterschiedliche Domänengrenzen angepasst werden, so dass diese Simulationen insgesamt vier freie Parameter besitzen. Beim natürlichen Perkolationsnetzwerk wurde die Stufenleitfähigkeit mikroskopisch bestimmt und als fester Parameter für die Transportsimulation verwendet (siehe Abschnitt 3.2.2). Im Gegensatz dazu, wurden bei der Analyse der vizinalen Oberfläche die Widerstandsverhältnisse derart skaliert, dass die simulierte mittlere Stromdichte der experimentellen Stromdichte entsprach. ${ }^{24}$ Abbildung 58 (c, e) zeigt die Ergebnisse für beide Stromrichtungen. Die Übereinstimmung der berechneten Potentiallandschaften mit den gemessenen Potentiallandschaften ist exzellent, so dass auch diese experimentellen Daten sich sehr gut im Rahmen der entwickelten Modellierung beschreiben lassen. Eine Zusammenfassung der Simulationsparameter ist in Tabelle 2 zu finden.

Hervorzuheben ist, dass sowohl die 2DEG-Leitfähigkeit als auch die monoatomare Stufenleitfähigkeit von der externen Stromrichtung abhängen und von den Ergebnissen des natürlichen Perkolationsnetzwerks abweichen. Zur Diskussion dieser Abweichungen müssen zusätzliche physikalische Prozesse berücksichtigt werden. Zur Klärung dieses Sachverhalts wird zunächst die unterschiedliche Transmission über die Terrassenstufen diskutiert. Daran anschließend wird die veränderte 2DEG-Leitfähigkeit betrachtet.

${ }^{24}$ Aufgrund der Kontaktgeometrie (punktförmige Kontaktierung mit zwei Goldspitzen) musste die Stromdichte am Ort der STP-Messungen aus der Analyse der elektrischen Feldverteilung auf der Mittelsenkrechten bestimmt werden. Eine Beschreibung dieses Verfahrens ist in Ref. [119] zu finden. 


\begin{tabular}{|c|c|c|c|}
\hline & $\begin{array}{c}\text { vizinales Substrat } \\
\text { jextern senkrecht } \\
\text { zur Stufe }\end{array}$ & $\begin{array}{l}\text { vizinales Substrat } \\
\text { Jextern parallel zur } \\
\text { Stufe }\end{array}$ & $\begin{array}{c}\text { Natürliches } \\
\text { Perkolationsnetzwerk }\end{array}$ \\
\hline$\sigma_{2 D E G}[\mu S / \square]$ & $>2000$ & $270+/-70$ & $3080+/-1100$ \\
\hline $\begin{array}{l}\text { Monoatomare } \\
\text { Stufe }\left[\Omega^{-1} m^{-1}\right]\end{array}$ & $3200+/-500$ & $<300$ & 5000 \\
\hline $\begin{array}{l}\text { Mehrfachstufen } \\
{\left[\Omega^{-1} m^{-1}\right]}\end{array}$ & $700+/-300$ & $<40$ & $\mathrm{x}$ \\
\hline $\begin{array}{l}\text { Domänengrenze } \mathbf{A} \\
{\left[\Omega^{-1} m^{-1}\right]}\end{array}$ & $3500+/-1000$ & $15+/-5$ & $\mathrm{x}$ \\
\hline $\begin{array}{l}\text { Domänengrenze B } \\
{\left[\Omega^{-1} m^{-1}\right]}\end{array}$ & $>10000$ & $1900+/-500$ & $\mathrm{x}$ \\
\hline
\end{tabular}

Tabelle 2: Zusammenfassung der spezifischen Leitfähigkeiten für Querstromrichtungen senkrecht und parallel zur präferierten Stufenrichtung. Bei senkrechter Stromrichtung zur präferierten Stufenrichtung kann die 2DEG-Leitfähigkeit nicht eindeutig bestimmt werden, da

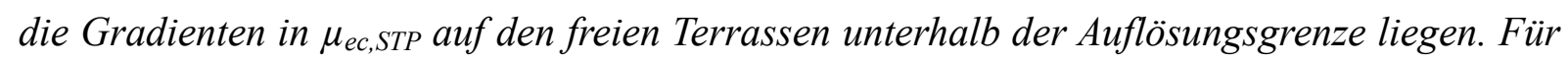
2DEG-Leitfähigkeiten größer als $2000 \mu \mathrm{S} / \square$ ergibt sich augenscheinlich keine Veränderung der Potentiallandschaft mehr, so dass nur eine untere Schwelle für die 2DEG-Leitfähigkeit angegeben werden kann. Für die Domänengrenze B kann ebenfalls nur eine untere Schwelle angegeben werden, denn sie liegt meist senkrecht zur externen Stromrichtung. Auflösungsbedingt sind daher kaum Potentialabfälle zu beobachten, so dass die numerische Anpassung dieser Leitfähigkeit kaum Auswirkungen auf die Potentiallandschaft hat. Bei paralleler Stromrichtung zur präferierten Stufenrichtung können aufgrund derselben Argumentation nur obere Grenzwerte für die Stufenleitfähigkeiten angegeben werden. Zum Vergleich sind die Resultate für das natürliche Perkolationsnetzwerk aus Kapitel 3.2.2.2 mit aufgeführt. 
Die Transmission durch die Barriere einer monoatomaren Stufe ist für den Ladungstransport senkrecht zur Stufenkante um den Faktor 10 höher als für den Ladungstransport parallel dazu. Begreift man den Transportprozess als quantenmechanische Transmission von ebenen Wellen des 2DEG über eine Potentialbarriere, so bedeutet eine senkrechte Querstromrichtung, dass der Wellenvektor senkrecht auf der Barriere steht. Beim parallelen Ladungstransport ist der Wellenvektor dementsprechend parallel zur Barriere. Vom Grundgedanken her kann im Rahmen der klassischen Mechanik verstanden werden, warum die Transmission bei senkrechtem Einfall maximal ist: Klassisch wäre zu erwarten, dass ein Teilchen mit der kinetischen Energie $E_{k i n}=1 / 2 m\left(v_{\perp}^{2}+v_{\|}^{2}\right)$ eine Barriere (z. B. eine schiefe Ebene) am besten überwinden kann, wenn sein Impulsvektor senkrecht zur Barriere zeigt. Für eine treffendere Beschreibung ist es notwendig, die quantenmechanische Transmission über eine Barriere als Funktion des Winkels zu betrachten. Analytisch kann in zwei Dimensionen die Transmission durch eine linienartige, unendlich ausgedehnte und rechteckige Barriere der Breite 2a und Potentialhöhe V berechnet werden zu

$$
\mathrm{T}=\mathrm{e}^{-2 \mathrm{ik}_{\mathrm{x}} \mathrm{a}} \frac{2 \mathrm{qk}_{\perp}}{2 \mathrm{qk}_{\perp} \cdot \cosh (2 \mathrm{qa})+\mathrm{i}\left(\mathrm{k}_{\perp}^{2}-\mathrm{q}^{2}\right) \sinh (2 \mathrm{qa})}
$$

mit $\mathrm{q}=\sqrt{2 \mathrm{~m}^{*}\left(\mathrm{~V}-\mathrm{E}_{\mathrm{f}}\right) / \hbar^{2}+\mathrm{k}_{\|}^{2}}$ und der Wellenvektorzerlegung $|\vec{k}|^{2}=k_{\perp}^{2}+k_{\|}^{2}$ in parallele und senkrechte Anteile zur Barriere. Bei der Herleitung wird die Tatsache, dass der Wellenvektor $k_{\|}$aufgrund der Konstruktion erhalten bleibt, ausgenutzt. Abbildung 59 zeigt die normalisierte Transmission $|T|^{2}$ als Funktion des Winkels der einlaufenden Welle für verschiedene Modellparameter, wobei Ref. [114] bei der Berechnung der Stufentransmission von 4.5 eV Barrierenhöhe und 2,35 $\AA$ Barrierendicke ausgeht. Allen Modellparametersätzen ist gemeinsam, dass die Transmission mit zunehmendem Winkel vom senkrechten Einfall abnimmt. Bei $30^{\circ}$ beträgt die Transmission bspw. noch $50 \%$, jedoch bei $70^{\circ}$ nur noch $0.1 \%$ der Transmission vom senkrechten Einfall. Diese erklärt die beobachtete Variation der Transmission um den Faktor 10 für die externen Stromrichtungen parallel und senkrecht zu den Stufenkanten. 


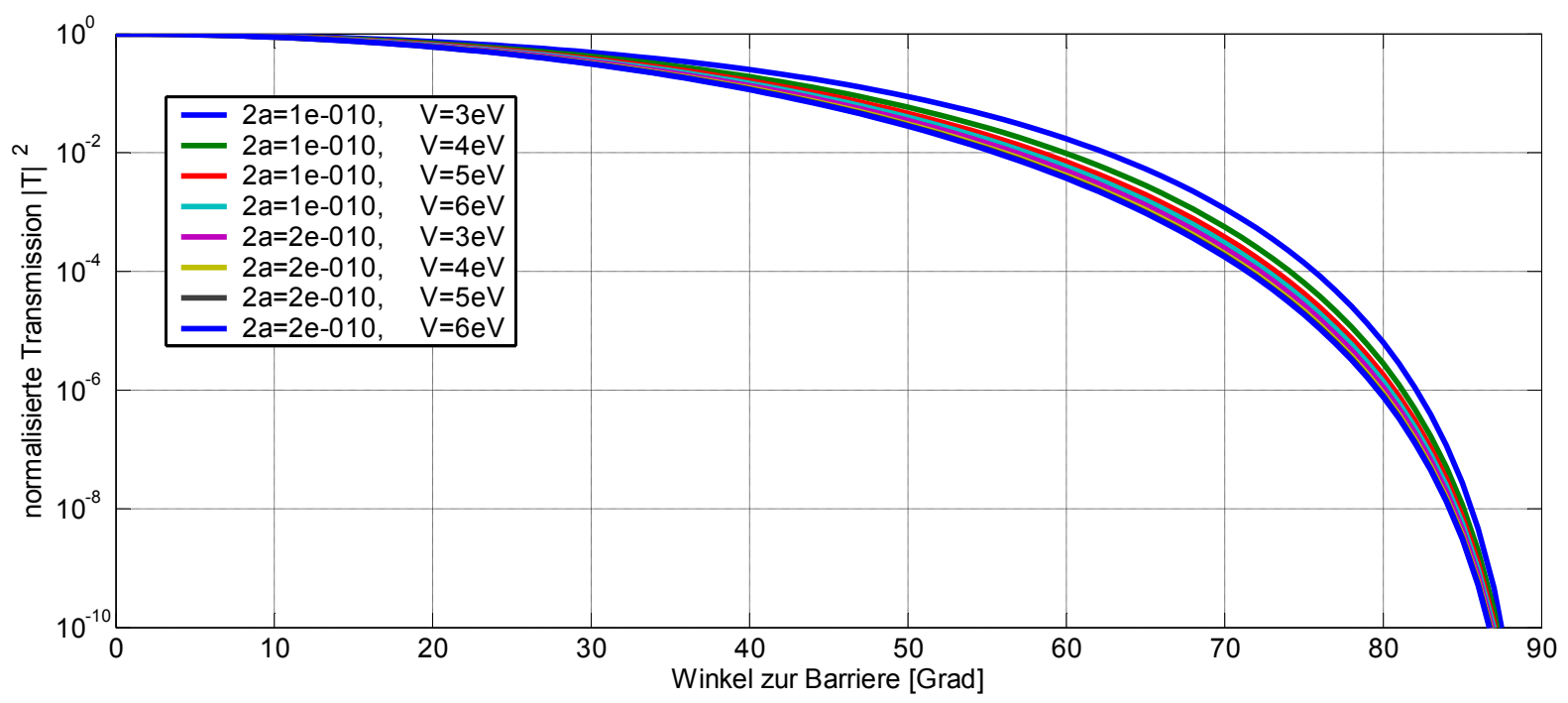

Abbildung 59: Normalisierte Transmission $|T|^{2}$ für verschiedene Parameter aus Barrierenhöhe und-Dicke als Funktion des Wellenvektor-Winkels zur Barriere. $0^{\circ}$ entspricht einem senkrechtem Einfall und bei $90^{\circ}$ ist die einlaufende Welle parallel zur Barriere. Die Berechnungen wurden ausgeführt für $k_{f}=2 \pi / 68$ A und $m^{*}=0.13 m_{e}$ entsprechend der experimentellen Werte aus Abschnitt 2.3.

Nachdem das Verhalten der Transmission über die Terrassenstufen analysiert und als Funktion der Stromrichtung interpretiert worden ist, soll nun die Variation der 2DEG-Leitfähigkeit diskutiert werden. Die 2DEG-Leitfähigkeit für senkrechte Querstromkonfiguration ist vergleichbar mit der 2DEG-Leitfähigkeit des natürlichen Perkolationsnetzwerkes aus Abschnitt 3.2.2.2. Erfolgt der Stromfluss jedoch entlang der engen Terrassen in der parallelen Querstromkonfiguration, so erscheint die 2DEG-Leitfähigkeit um den Faktor 10 vermindert. Die Ursache für diese verminderte Leitfähigkeit interpretieren wir als laterale Grenzflächenstreuung. ${ }^{25}$ Die mittlere freie Weglänge wurde in Abschnitt 4.2 anhand der Ergebnisse auf dem natürlichen Perkolationsnetzwerk zu $70 \mathrm{~nm}$ abgeschätzt, was vergleichbar mit der Terrassenbreite ist. In einem semiklassischen Transportbild erfahren die Ladungsträger demnach eine Streuung an den lateralen Grenzflächen. Eine verminderte effektive freie Weglänge beim Transport entlang der Kanäle ist die Folge. Dies ist analog zur Reduktion der Leitfähigkeit in dünnen Filmen durch Oberflächenverunreinigungen wie sie von Fuchs und Sondheimer 1952 gefunden worden ist [120].

\footnotetext{
${ }^{25}$ In zwei Dimensionen könnte man laterale Grenzflächen auch Grenzlinien nennen.
} 


\subsection{Diskussion der mikroskopischen Transporteigenschaften}

Die Modellierung des Ladungstransports hat sich als ein funktionelles Instrument erwiesen, um einen Zugang zu den mikroskopischen Transporteigenschaften zu erlangen. Gerade im Hinblick auf die in Abschnitt 3.3 vorgestellten Ergebnisse zeigt sich jedoch, dass eine Beschreibung des Ladungstransports mit konstanten mikroskopischen Größen in beliebigen Strukturen unzureichend sein kann: Durch laterale Grenzflächenstreuung kann die effektive Leitfähigkeit auf einer Terrasse in engen Kanälen vermindert sein und die Transmission über eine Terrassenstufe ist im Allgemeinen eine winkelabhängige Funktion.

Im Zuge dieser Resultate deuten wir das Ergebnis für die Stufenleitfähigkeit des natürlichen Perkolationsnetzwerks $\left(\sigma_{\text {Stufe }}=5000 \Omega^{-1} m^{-1}\right)$ als Mittelwert der Transmissionsfunktion über alle Einfallswinkel. Das Ergebnis für die Stufenleitfähigkeit bei präferierter Stromrichtung senkrecht $\mathrm{zu}$ den Stufen $\left(\sigma_{\text {Stufe, } \perp}=3200 \Omega^{-1} m^{-1}\right)$ ist demnach als eine Mittelung der Transmissionsfunktion für kleine Winkel zu interpretieren. Eigentlich sollte die Leitfähigkeit bei präferiert senkrechtem Einfall größer sein als bei der Mittelung im natürlichen Perkolationsnetzwerk. Dies ließ sich mit den durchgeführten Experimenten jedoch nicht eindeutig verifizieren. Eine Erklärung dieser Diskrepanz könnte in einer geringeren Ag-Adatom Dotierung und damit geringeren Ladungsträgerdichte im 2DEG bei den Duisburger-Proben zu finden sein.

Die Bestimmung der 2DEG-Leitfähigkeit auf dem natürlichen Perkolationsnetzwerk der $\operatorname{Si}(111)(\sqrt{3} x \sqrt{ } 3)-A g$ Rekonstruktion ist wahrscheinlich nicht durch laterale Grenzflächenstreuung beeinflusst, da die Strukturgrößen meist oberhalb der mittleren freien Weglänge liegen. Zusätzlich sollte das „ungeordnete“ Perkolationsnetzwerk, im Gegensatz zur kanalartigen Terrassenstruktur auf den vizinalen Oberflächen, weniger anfällig für Effekte durch Grenzflächenstreuung sein. Die 2DEG-Leitfähigkeit des natürlichen Perkolationsnetzwerks $\sigma_{2 D E G}=3080 \mu \mathrm{S} / \square$ wird damit als die intrinsische Leitfähigkeit des 2DEG bei Raumtemperatur interpretiert. 


\section{Das elektrochemische Potential in der Umgebung von lokalisierten Defekten}

Sowohl von theoretischer als auch experimenteller Seite her ist bisher wenig bekannt, wie sich das elektrochemische Potential direkt an einer stromdurchflossenen Barriere verhält.

Für homogene Systeme im Gleichgewicht kann eine Verteilungsfunktion gemäß $f(k)$ definiert werden. Für homogene System im Nicht-Gleichgewicht ist eine Beschreibung in thermodynamischen quasi-Gleichgewichtsgrößen zweckmäßig und eine Beschreibung gelingt mit kabhängigen Verteilungsfunktionen $f(\vec{k})$. In einer Dimensionen führt dies zu zwei quasiVerteilungsfunktionen für die jeweiligen Transportrichtungen $f\left(k^{+}\right)$und $f\left(k^{-}\right)$, wie sie schematisch in Abschnitt 1.2.2 diskutiert worden sind.

Bei inhomogenen Systemen im Nicht-Gleichgewicht kann die Besetzungsfunktion jedoch ortsabhängig werden $f(\vec{k}, \vec{r})$, was eine konzeptionelle Beschreibung bis heute erschwert. Soll der Ladungstransport darüber hinaus auf Längenskalen kleiner als die Phasenkohärenzlänge $l_{\phi}$ und die Energierelaxationslänge beschrieben werden, so kann eine Beschreibung mit thermodynamischen quasi-Gleichgewichtsgrößen unzureichend sein und eine Beschreibung müsste vollständig quantenmechanisch unter Berücksichtigung der inhomogenen NichtGleichgewichtsverteilung stattfinden. Dies erschwert eine geschlossene Beschreibung bis heute.

Die durchgeführten STP-Messungen erlauben erstmalig, den Ladungstransport mit höchster räumlicher Auflösung in einem 2D-System zu studieren. Durch die niedrige Dimensionalität sind dabei die Ladungsdichte nahe der Fermienergie und die Defektstruktur als Topographie zugänglich. Ferner erhält man über die Messgröße $\mu_{e c, S T P}$ ein Maß für das elektrochemische Potential, welches die lokalen Besetzungsfunktionen des Nicht-Gleichgewichts gemittelt über die Energie beinhaltet. Die Besetzungsfunktionen werden durch Streuprozesse und Abschirmung auf unterschiedlichen Längenskalen dynamisch bestimmt. In diesem Kapitel wird experimentell der räumliche Verlauf des elektrochemischen Potentials an einer monoatomaren Stufe mit atomarer Auflösung untersucht und die gemessene Ausdehnung der Variation im Rahmen der angesprochen Konzepte (siehe Abschnitt 1.2.2) diskutiert. 
Der räumliche Verlauf des elektrochemischen Potentials wurde anhand von hochauflösenden STP-Daten mit einer Rasterweite von bis zu $2 \AA$, entsprechend Abbildung 60 analysiert. Der gewählte Probenort beinhaltet eine nahezu streifenförmige Terrasse. Drei elektrisch voneinander entkoppelte Bereiche, die jeweils durch eine aufsteigende und absteigende Terrassenstufe definiert werden, können mit dieser Auflösung simultan untersucht werden. Die externe Stromrichtung wurde zwischen der Hin- und Rückspur einer jeden Rasterzeile umgedreht, um Effekte, die ausschließlich mit der Stromrichtung verknüpft sind, herausarbeiten zu können. Eine laterale Drift zwischen beiden Stromrichtungen wird aufgrund der schnellen zeitlichen Abfolge minimiert. Einzelschnitte aus den Topographie und Potentialdaten sind für beide Stromrichtungen und Terrassenflanken in Abbildung 61 dargestellt. (a) zeigt einen Einzelschnitt für eine ansteigende und (b) für eine abfallende Terrassenkante.

a)
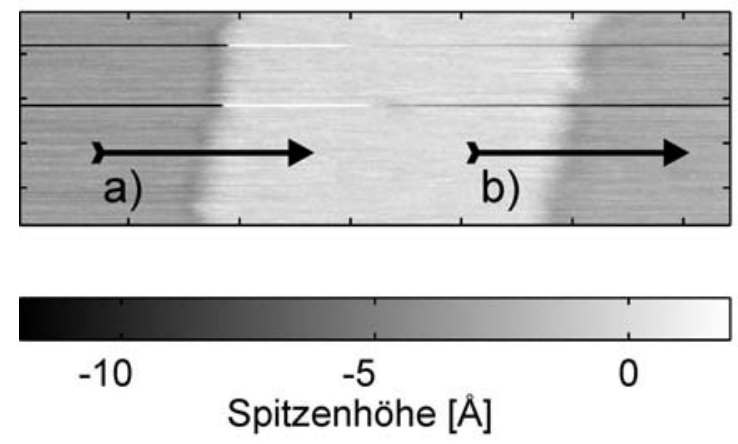

c)
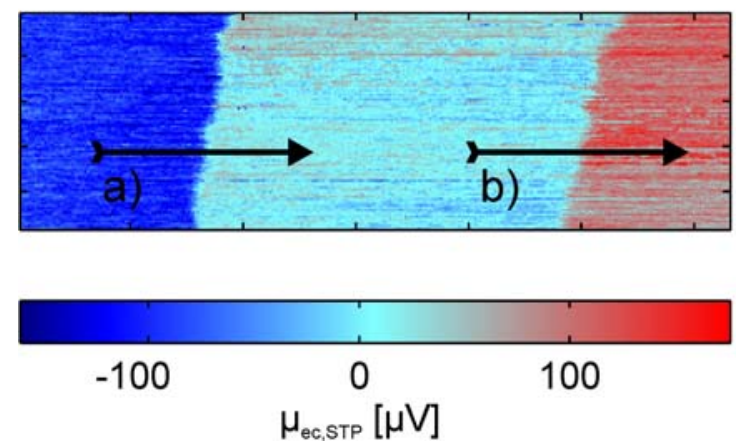

b)
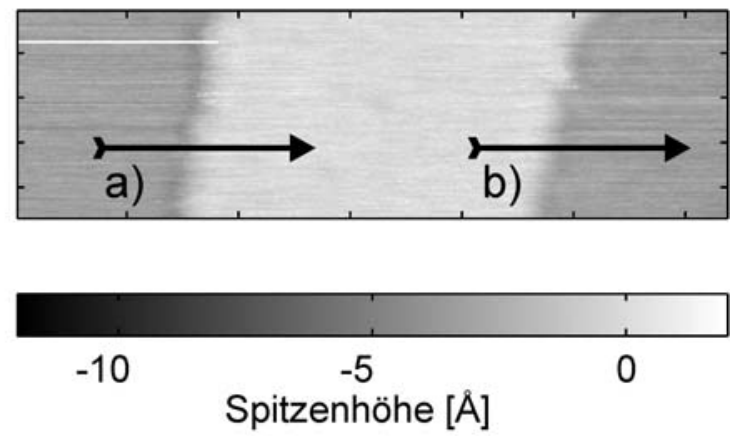

d)
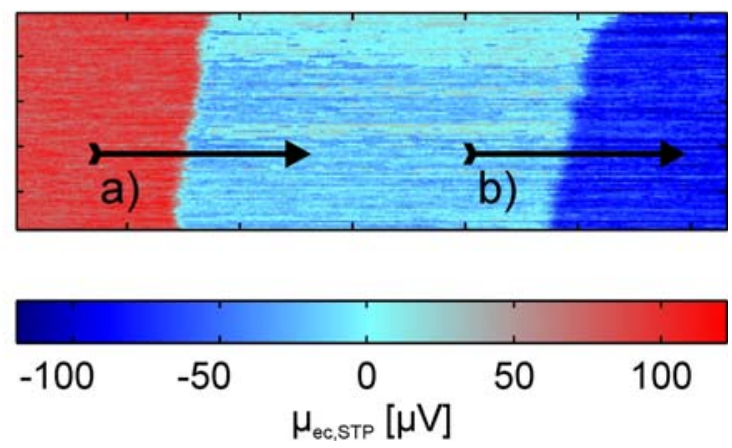

Abbildung 60: Hochaufgelöste STP-Daten an Stufenkanten $\left(20 \times 60 \mathrm{~nm}^{2}\right)$. (a, b) zeigen die entsprechenden Topographien aufgenommen bei $I_{t}=1 \mathrm{nA}$ und $U_{\text {Bias }}=0,01 \mathrm{~V} .(\mathrm{c}, \mathrm{d})$ zeigen die simultan aufgenommen Potentiallandschaften $\mu_{e c, S T P}$ bei $j_{\text {extern }}=108 \mathrm{~mA} / \mathrm{m}$ (Hinspur) und (d) bei jextern $=75 \mathrm{~mA} / \mathrm{m}$ (Rückspur). Der Rasterabstand beträgt 2 A. Gestrichelte Pfeile in den Bildern markieren laterale Schnitte entlang der schnellen Rasterrichtung, entsprechend Abbildung 61. 


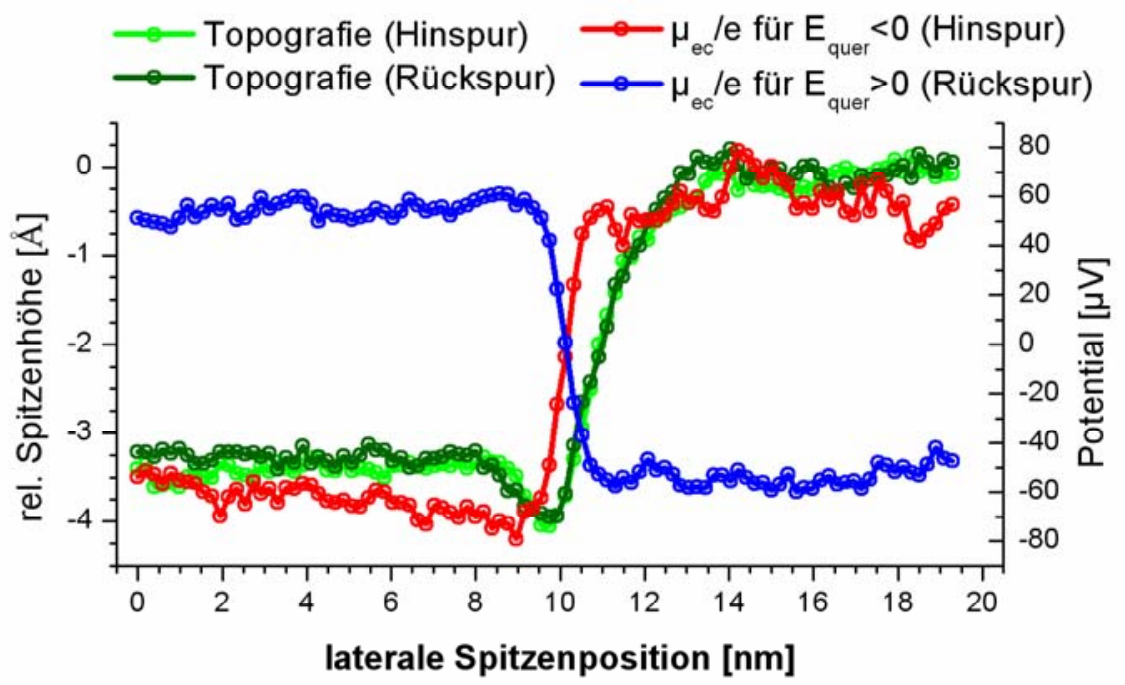

(a)

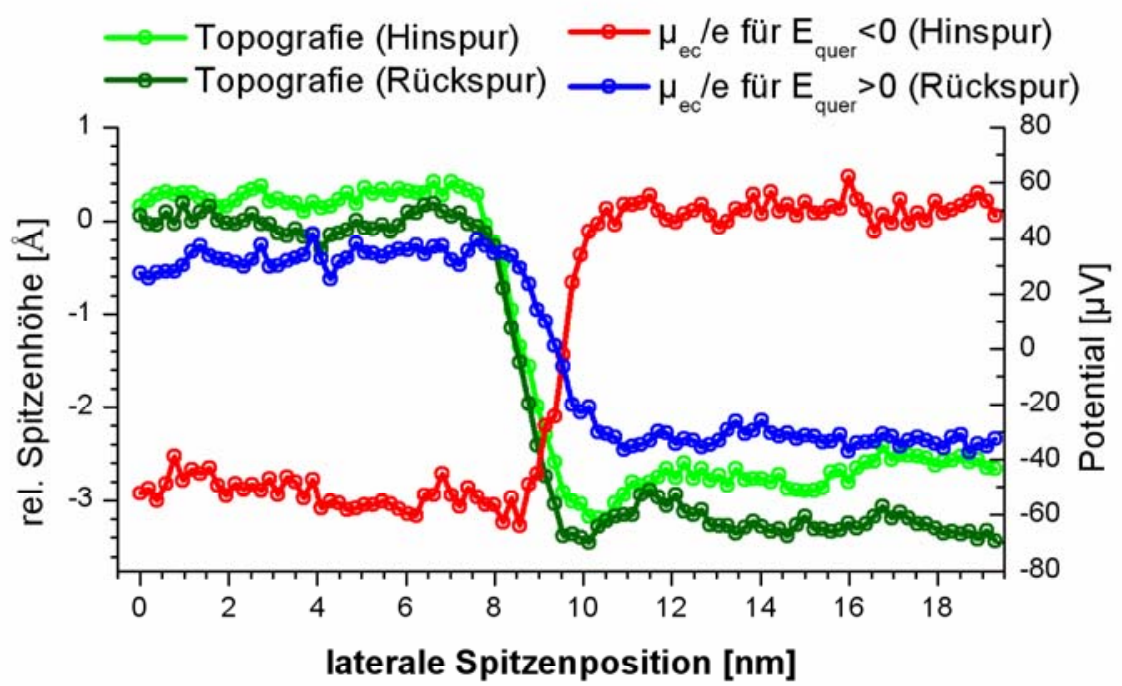

(b)

Abbildung 61: Analyse einer Rasterzeile gemäß Abbildung 60: (a) zeigt $\mu_{e c, S T P^{-}}$und Topographie-Daten für eine ansteigende und (b) für eine abfallende Terrassenstufe, jeweils für beide Stromrichtungen. Der Potentialsprung ist für beide Polaritäten auf ca. 7(2) A lokalisiert und die Stufenkante erstreckt sich über 14(2) A. Abbildung aus Ref. [36].

Die Topographie-Spur der Hin- und Rückspur und somit beider Stromrichtungen liegt sehr exakt übereinander. Eine laterale Drift ist somit kaum vorhanden und der räumliche Verlauf der STP-Daten beider Stromrichtungen kann absolut verglichen werden. Das Potential auf dem ungestörten 2DEG zeigt innerhalb der betrachteten Längenskala von $7 \mathrm{~nm}$ keinen Gradienten und variiert um ca. $20 \mu \mathrm{V}$. An der Stufenkante variiert $\mu_{\mathrm{ec}, \mathrm{STP}}$ monoton vom 


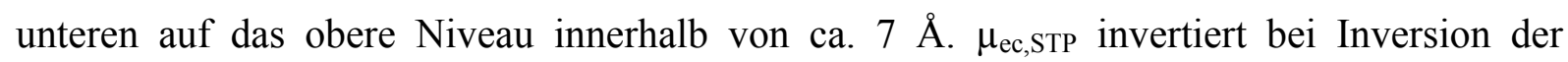
externen Stromrichtung symmetrisch um seinen Mittelpunkt. Die Topographie-Spur besitzt mit $14 \AA$ eine doppelt so große Übergangsweite wie die Potentialdaten. Auffallend ist eine Vertiefung auf der jeweils unteren Terrasse mit etwa $2 \mathrm{~nm}$ lateraler Ausdehnung. Vergleicht man an der Stufenkante die laterale Position der Topographie- und Potentiometrie-Spur bei jeweils 50\% ihres Anstiegs, so ergibt sich, dass der Potentialsprung nicht am Ort der Stufe lokalisiert ist. In Abbildung 61 beträgt der Versatz ca. $15 \AA$.

Dies sind überaschende Ergebnisse. Erstens sollte nach S. Datta (siehe auch Abschnitt 1.2.2.2) das elektrochemische Potential von einem Niveau auf das andere abrupt am Ort der Barriere variieren [39] und zweitens verwundert es, dass der Übergang in $\mu_{\mathrm{ec}, \mathrm{STP}}$ nicht mit der lateralen Position der Stufe zusammenfällt und an der die Tunnelbarriere lokalisiert ist [99].

Für eine signifikantere Analyse dieses Sachverhalts wurde eine Vielzahl von Messdaten statistisch ausgewertet. Hierzu wurden symmetrische Stufenfunktionen der Form $\mu_{e c, S T P}(x)=\Delta \mu_{\text {Stufe }} \cdot\left(1+\exp ^{\frac{\mathrm{x}-\mathrm{x}_{0}}{\Delta \mu}}\right)^{-1}+\mu_{\text {Offset }}$ numerisch angepasst. ${ }^{26}$ Die absolute Position $\left(\mathrm{x}_{0}\right)$, die Übergangsweite $\Delta \mu$ und die Variation $\Delta \mu_{\text {Stufe }}$ eines jeden Einzelschnitts konnten auf diese Weise mit voller Statistik der Messwerte ausgewertet werden. Dies erfolgte gleichsam für die Topographie und Potentiometrie. Abbildung 62 zeigt die entsprechenden Größen auf Basis einer Statistik von 356 Einzelschnitten. Die Verteilungen sind nahezu normalverteilt und die entsprechenden Standardabweichungen sind angegeben. Der Verlauf von $\mu_{\mathrm{ec}, \text { STP }}$ und der Topographie an der Stufenkante lässt sich aufgrund dieser Analyse mit drei Beobachtungen beschreiben: ${ }^{27}$

I. Die Variation von $\mu_{\mathrm{ec}, \mathrm{STP}}$ ist der Topographie-Variation um $\Delta \mathrm{X}=10.8(5) \AA$ zugunsten der unteren Terrasse vorgelagert.

II. An der Stufenkante variiert $\mu_{\mathrm{ec}, \mathrm{STP}}$ monoton vom unteren auf das obere Niveau innerhalb von $\mathrm{L}_{\Delta \mu}=5.6(4) \AA$.

III. Die Variation in der Topographie-Spur besitzt mit $\mathrm{L}_{\Delta \text { Stufe }}=10.5(4) \AA$ eine doppelt so große Übergangsweite wie die Potentialdaten.

\footnotetext{
${ }^{26}$ Anstelle von $\mu$ wird bei der Topografie Anpassung z verwendet.

${ }^{27}$ Die Übergangsweite wird hierbei als 20\% - 80\% Übergangsweite bestimmt.
} 
a)

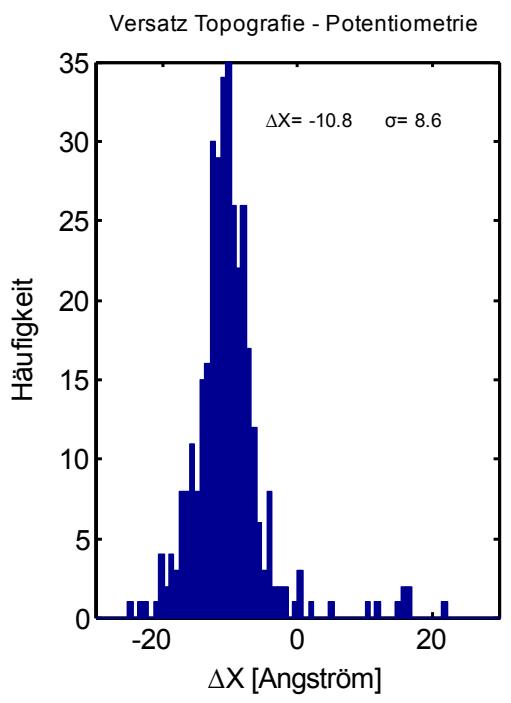

b)

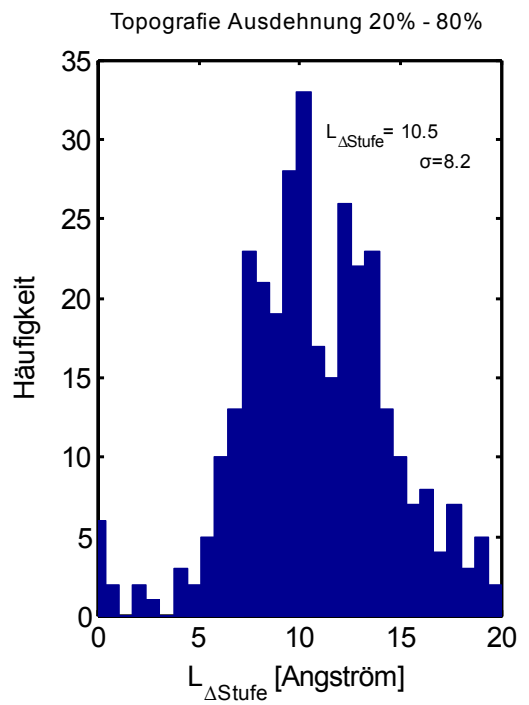

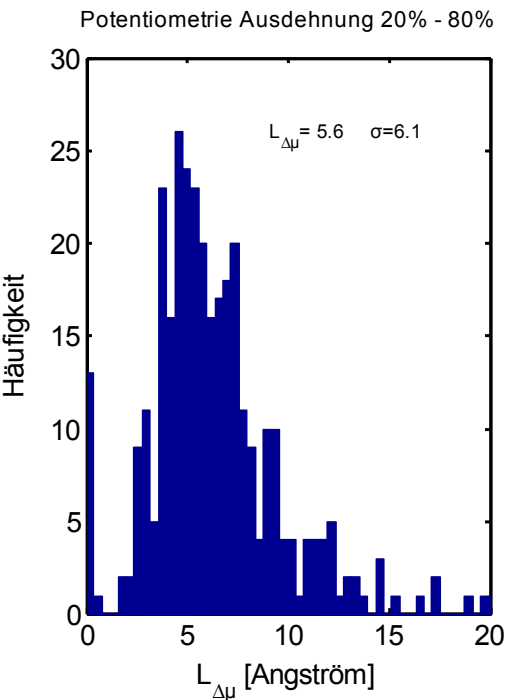

Abbildung 62: (a) Zeigt die Verteilung des Versatzes $\triangle X$ zwischen der Variation in der Topographie und Potentiometrie. (b) Illustriert die Übergangsweiten der Topographie-Spur $L_{\Delta S t u f e}$ und (c) die Übergangsweiten in der Potentiometrie-Spur $L_{\Delta \mu}$. Der Medianwert der Verteilung und die entsprechenden Standardabweichungen sind angegeben. Bei angenommener Normalverteilung sind die gesuchten Größen: (a) Versatz von $\mu_{e c, S T P}$ zur Topographie zugunsten der unteren Terrasse $\triangle X=10.8(5) \AA$, (b) Übergangsweite der Topographie $L_{\Delta S t u f e}=10.5(4)$ A und (c) Übergangsweite von $\mu_{e c, S T P} L_{\Delta \mu}=5.6(4)$ A. Insgesamt wurden 356 Einzelschnitte ausgewertet.

Die Positionen der lateralen Variationen der $\mu_{\mathrm{ec}, \mathrm{STP}}$-Daten und der Topographie sind durch die Anpassung der Fit-Funktion absolut bekannt. Die Einzelschnitte an einer im Allgemeinen nicht gradlinigen Terrassenstufe können dadurch „gerade gezogen“ werden, um sie anschließend über einen weiten Bereich lateral zu mitteln. Abbildung 63 illustriert das Resultat und zeigt die Qualität der geradlinigen Ausrichtung. Zwei so nachbearbeitete Datensätze, bestehend aus je 100 Rasterzeilen, sind dargestellt. (c, f) zeigt die mittleren Verläufe von $\mu_{\mathrm{ec}, \mathrm{STP}}$ und der Topographie aus einem Probenbereich von jeweils $14 \mathrm{~nm}$ senkrecht zur Stufe. 

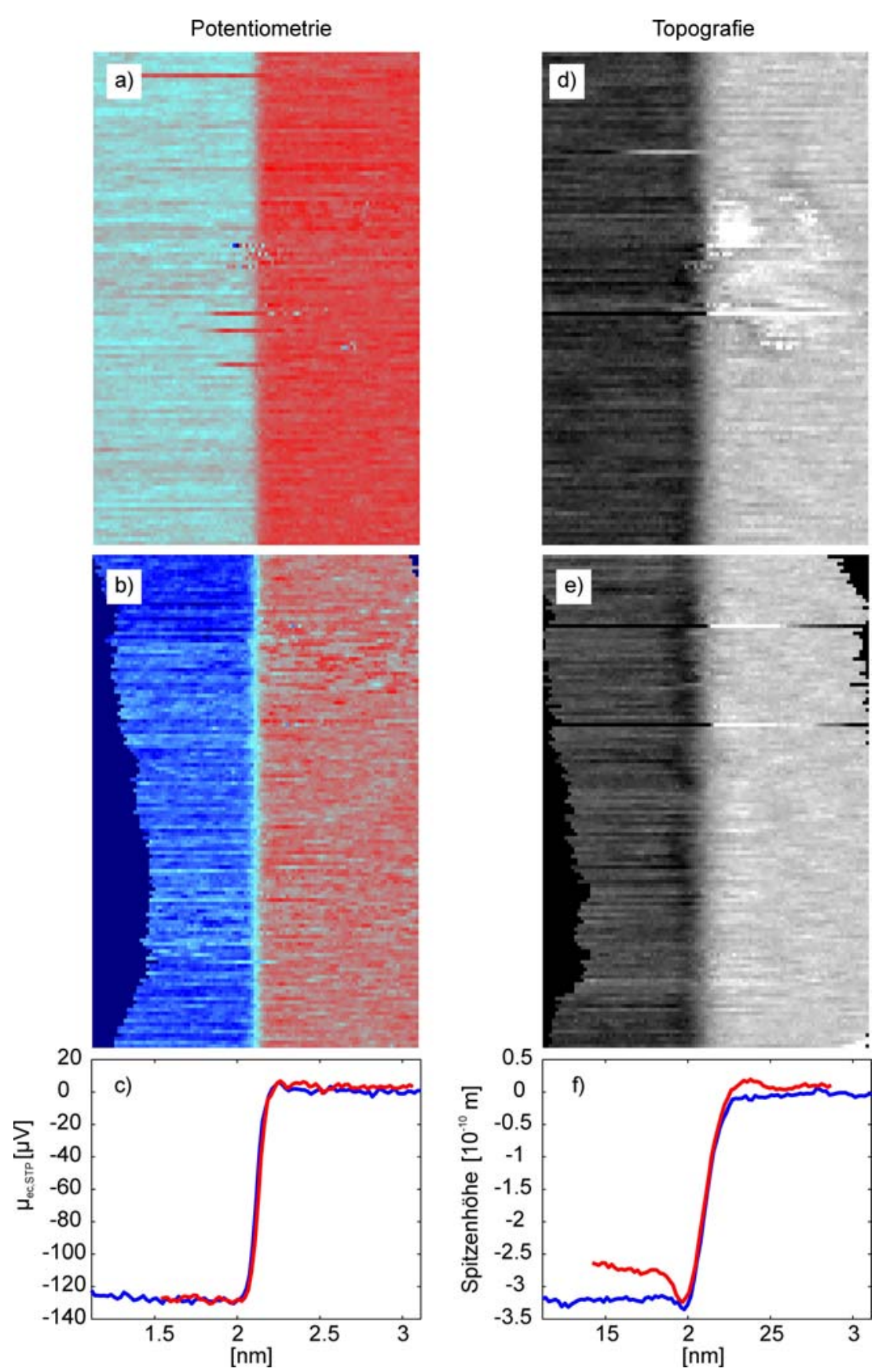

Abbildung 63: Aus der Analyse gemäß Abbildung 62 ist die Position des Sprungs in der Potentiometrie und der Topographie bekannt. Jede Rasterzeile kann dadurch lateral verschoben werden, so dass eine gerade Kante entsteht. $(a, b)$ zeigt die ausgerichteten Potentiometrie-Daten und $(d, e)$ die Topographie-Daten aus zwei unterschiedlichen Bereichen von jeweils $(14 \times 10) \mathrm{nm}^{2}$. Die ausgerichteten Zeilen können dadurch absolut senkrecht zur Terrassenstufe über einen weiten Bereich gemittelt werden. (c) Das Potentiometrie-Signal ist in guter Näherung eine ausgeschmierte Stufenfunktion der Breite $L_{\Delta \text { Stufe }}=10.5$ A. (f) Das Topographie-Signal besitzt ein Minimum von $\sim 30$ pm Tiefe vor der Stufe auf der unteren Terrasse. Nahe der Auflösungsgrenze ist ein vorgelagertes Maximum zu erkennen. Eine symmetrische Oszillation auf der oberen Terrasse ist im roten Datensatz (e) zu erkennen. 
Der Verlauf von $\mu_{\mathrm{ec}, \mathrm{STP}}$ entspricht den vorherigen Beobachtungen hinsichtlich Symmetrie und Übergangsweite. In der Topographie (f) ist nun sehr deutlich eine Vertiefung von $\sim 30$ pm auf der unteren Terrasse zu erkennen. Nahe an der Auflösungsgrenze ist ein vorgelagertes Maximum erkennbar. Eine symmetrische Oszillation auf der oberen Terrasse ist im Datensatz (e) auszumachen.

Bisher gibt es keine vergleichbaren experimentellen Daten, welche die Vertiefung bzw. die Oszillation auf der unteren und ggf. auch oberen Terrasse in der Topographie beschreiben. Es ist jedoch naheliegend, die Oszillationen an der Terrassenkante als Friedel-Oszillationen zu interpretieren, welche mit Hilfe von STS in der LDOS bei tiefen Temperaturen bereits intensiv studiert worden ist $[99,121]$. Die ursprünglich ungestörte Ladungsträgerdichte sollte demnach mit $\lambda_{\mathrm{F}} / 4$ oszillieren und ist innerhalb von $\lambda_{\mathrm{F}} / 4 \mathrm{um}$ die Barriere vermindert. Nach Kapitel 2.3.1 beträgt die Fermiwellenlänge auf dieser Probe $\lambda_{\mathrm{F}}=68 \AA$ (aus $\mathrm{E}_{\mathrm{f}}$ und $\mathrm{m}^{*}$ ), so dass sich die theoretische Ausdehnung der Vertiefung in der Topographie zu $17 \AA$ ergibt, was dem experimentellen Befund entspricht.

Nachdem die Topographie-Spur ausreichend diskutiert und verstanden worden ist, bleibt noch zu klären, warum die Variation in $\mu_{\mathrm{ec}, \mathrm{STP}}$ der Terrassenstufe vorgelagert ist und ob die beobachtete Übergangsweite in $\mu_{\mathrm{ec}, \mathrm{STP}}$ mit der vorherrschenden Lehrmeinung eines abrupten Übergangs von $\mu_{\mathrm{ec}}$ konform ist. Um diesen Sachverhalt zu klären, wurde eine Simulation zur Raster-Tunnel-Potentiometrie angefertigt, die im nächsten Kapitel vorgestellt wird. Im Anschluss daran werden die Ergebnisse der Simulation im Hinblick auf eine Interpretation der gemachten Beobachtungen dargestellt. 


\subsection{Simulation der STP-Daten über einer Stufenkante}

Die gemessenen Verläufe der Topographie- und Potentiometrie-Daten (Übergangsweiten sowie ein möglicher Versatz zwischen der Topographie und $\mu_{\mathrm{ec}, \mathrm{STP}}$ ) sind durch die Abbildung mit einer Sonde (Tunnelspitze) endlicher Ausdehnung beeinflusst. Um den Einfluss von Abbildungsartefakten abschätzen zu können, wurde eine STP-Simulation geschrieben, welche sowohl Topographie- als auch $\mu_{\mathrm{ec}, \mathrm{STP}}$-Daten berechnet.

Im Rahmen dieser Simulation wird die $\vec{k}$-Abhängigkeit der Transmission vernachlässigt. Es wird angenommen, dass der Tunnelstrom in einfacher Weise exponentiell vom Abstand $d_{t}=\sqrt{\Delta z^{2}+\Delta y^{2}+\Delta x^{2}}$ zwischen einem Aufpunkt der Spitze zu einem Aufpunkt der Oberfläche abhängt. Der Tunnelstrom zwischen zwei Aufpunkten ist somit einfach $I_{t}=e^{-\kappa d_{t}}$ mit $\kappa=\ln (10) \AA^{-1}$, der häufig verwendeten Abklingkonstanten für STM-Experimente im UHV. Die Sondenspitze wird ebenso wie die Oberfläche aus diskreten Aufpunkten modelliert. Als Diskretisierung hat sich $1 \AA$ in der xy-Ebene als zweckmäßig und ausreichend genau erwiesen. Der z-Abstand in der Tunnelspitze wird auf $1.82 \AA$ dem W(111)Ebenenabstand gesetzt, da Wolframdraht mit $<111>$-Vorzugsorientierung verwendet wurde. Abbildung 64 verdeutlicht dies in einer maßstabsgetreuen 2D-Ansicht.

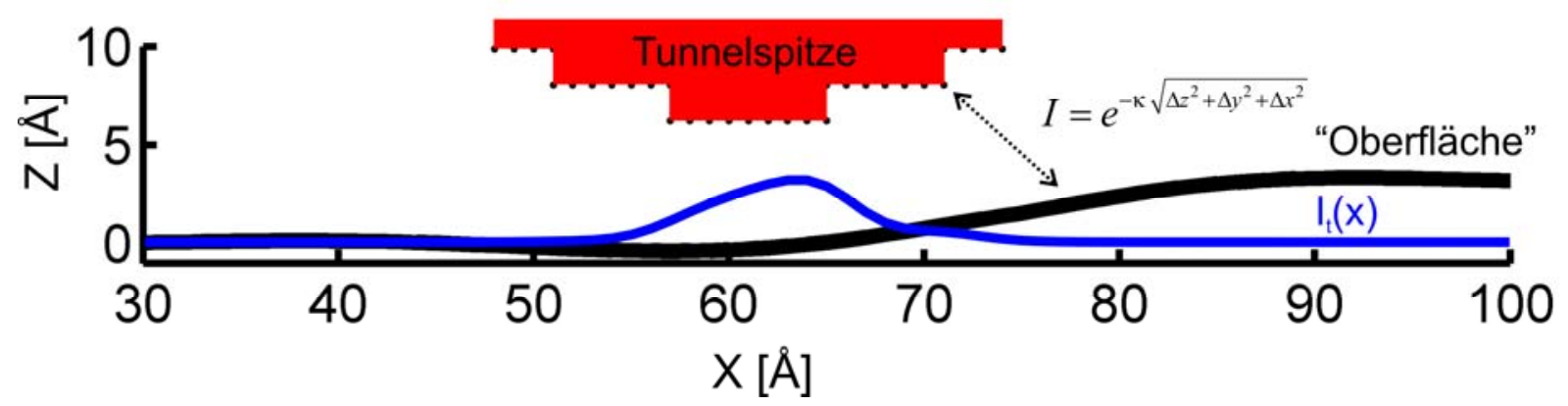

Abbildung 64: 2D-Darstellung zur Illustration der STP-Simulation. Nur die Aufpunkte der Spitze mit einem Durchmesser von 25 A sind gezeigt. Der Tunnelabstand beträgt 7 A. I $I_{t}(x)$ zeigt die Tunnelstromdichte in willkürlichen Einheiten am Ort der Probe für die gezeigte Spitzenposition. Die gezeigte Situation ist in Abbildung 65 in einer 3D-Darstellung zu sehen.

Der gesamte Tunnelstrom ergibt sich aus der Summe aller Aufpunkte der Spitze zu jedem Aufpunkt der Oberfläche. Um Rechenzeit zu sparen, wird die Summe eines jeweiligen Spitzenpunktes jedoch nur zu Probenpunkten innerhalb von 30 x $30 \AA^{2}$ in der xy-Ebene ausgeführt. Der Tunnelstrom wird beim Rastern über die Oberfläche durch Nachführen der 
Spitzenhöhe bis auf 1\%o konstant gehalten, was der Messvorschrift der Konstant-StromTopographie entspricht. Die Höhenangabe bezieht sich auf den Abstand des letzten Aufpunkts der Spitze zum nächstgelegenen Aufpunkt der Oberfläche. Der Tunnelstromsollwert wird hierfür durch Setzen der Sondenhöhe über einer Topographie konstanter Höhe zu Anfangs einmalig bestimmt.

Abbildung 65 zeigt eine 3D-Darstellung der Ergebnisse mit einer Spitze von $25 \AA$ Durchmesser über einer Modelloberfläche im Tunnelabstand von $7 \AA$. Aufgrund der ansteigenden Topographie verschiebt sich der Tunnelstrompfad zu positiven x-Werten im Vergleich zum

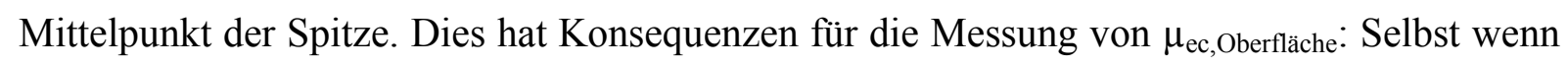
die Spitze topographisch noch vor der Stufe ist, kann bereits ein Teil des Tunnelstroms aus dem Bereich der eigentlichen Stufe kommen. Es ist daher verständlich, dass eine Variation von $\mu_{\mathrm{ec}, \mathrm{STP}}$ vor der Variation in der Topographie möglich ist.

Auf Basis dieser Modellvorstellung kann $\mu_{\mathrm{ec}, \text { STP }}$ berechnet werden, um sowohl die Abbildungsschärfe als auch den Versatz zur Topographie-Variation bestimmen zu können. Die Berechnungen wurden derart ausgeführt, dass jedem Ort der Oberfläche ein spezifisches

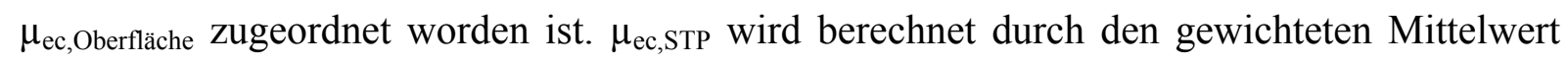
der lokalen $\mu_{\mathrm{ec}, \text { Oberfläche mit dem aus diesem Bereich kommenden Tunnelstrom. }}{ }^{28}$

${ }^{28}$ Eine energieunabhängige Transmission wurde in Kapitel 3 im relevanten Energieintervall für den elektrischen Transport experimentell bestätigt. 


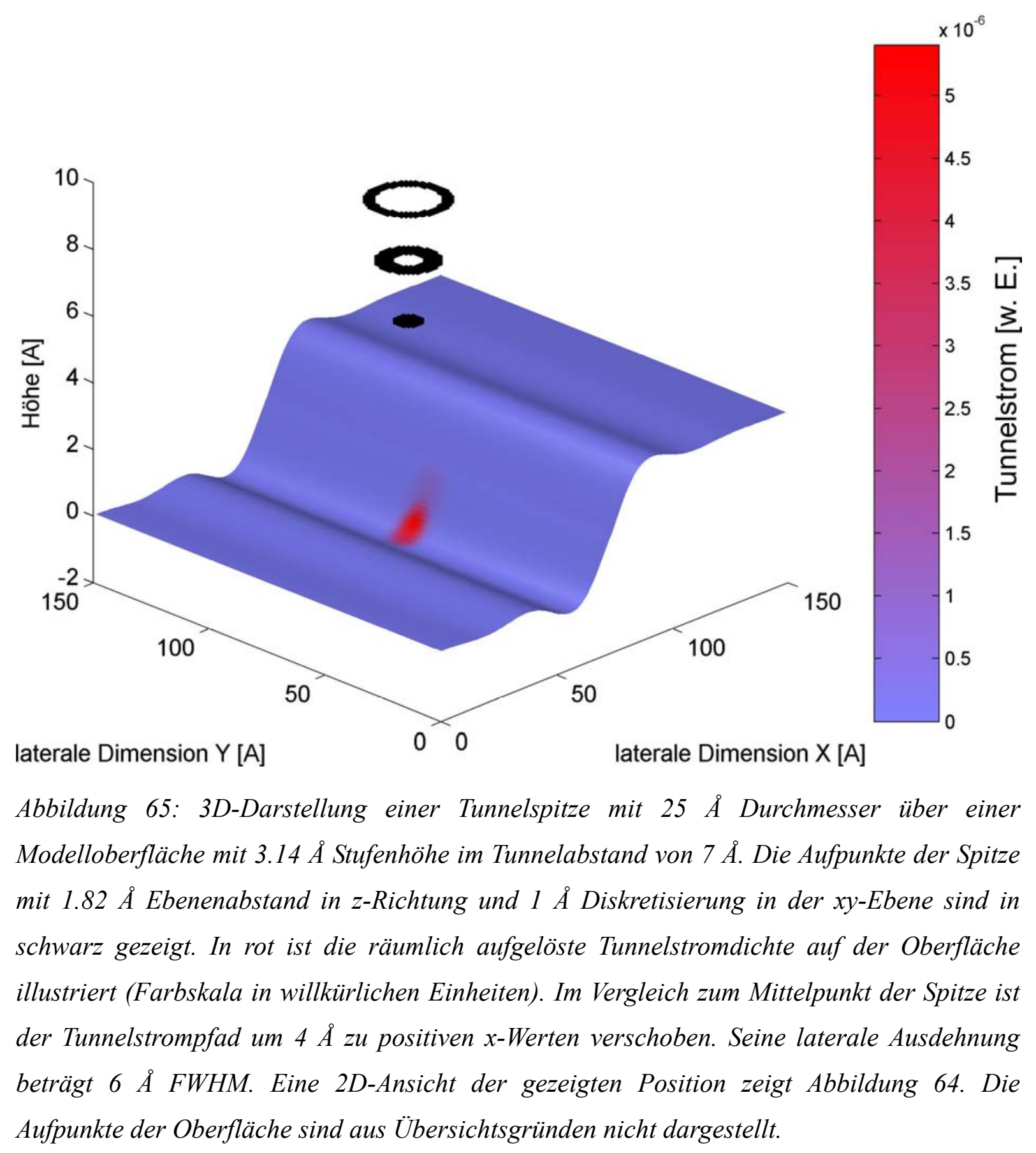




\subsection{Ergebnisse der Simulationen}

In diesem Abschnitt werden zunächst die am besten angepassten Resultate diskutiert. Zum besseren Verständnis werden erst am Ende Flussdiagramme der Parameterveränderungen (wie z. B. Spitzendurchmesser, Tunnelabstand, Verlauf von $\mu_{\mathrm{ec}, \text { Oberfläche }}$ und der Topographie etc.) und deren Einfluss auf die Übereinstimmung diskutiert. Zunächst muss jedoch die LDOS an

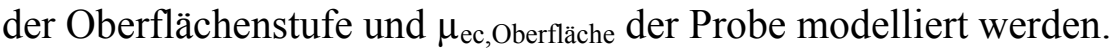

Angelehnt an einen DFT-LDA Ansatz von Lang und Kohn wird die LDOS an einer Oberflächenstufe durch „Friedel-Oszillation“, wie sie im Kapitel 4 bereits diskutiert worden ist, ausgeschmiert [122]. Die selbstkonsistente Abschirmung der Ladungen verhindert hierbei eine abrupte Variation der LDOS an der Terrassenstufe und sorgt für eine endliche Übergangsweite.

Praktisch wurde dies umgesetzt, indem die Friedel-Oszillation senkrecht zu einer unendlich ausgedehnten Stufe berechnet wurde. Die Stufe wird als $\delta$-Barriere mit einer Transmission T behandelt. Auf dem zweiten Halbraum der Stufe wird ebenfalls eine Friedel-Oszillation angewendet. Die Stufenhöhe wurde anschließend auf eine monoatomare $\operatorname{Si}(111)(\sqrt{3} x \sqrt{3})-\mathrm{Ag}$ Stufe (3.14 $\AA$ ) normiert. Um einen möglichen, unsymmetrischen Verlauf der Topographie modellieren zu können, wurde die Friedel-Oszillation auf beiden Terrassenseiten (Halbräumen) unterschiedlich stark exponentiell gedämpft. Analytisch bedeutet dies

$$
\begin{aligned}
& z=z_{\text {links }}+z_{\text {rechts }} \\
& z_{\text {links }}=\left.a_{\text {links }}\left(b_{\text {links }}-R J_{0}\left(-k_{F} x+\Phi\right) \cdot e^{\kappa_{\text {links }} x}\right)\right|_{x<0} \\
& z_{\text {rechts }}=\left.a_{\text {rechts }}\left(b_{\text {rechts }}-R J_{0}\left(k_{F} x+\Phi\right) \cdot e^{-\kappa_{\text {rechs }} x}\right)\right|_{x>0} \\
& \left.z_{\text {links }}\right|_{x=0}=\left.z_{\text {rechts }}\right|_{x=0} \\
& \max (z)-\min (z)=3.14 \dot{A}
\end{aligned}
$$

mit $\mathrm{J}_{0}$ der Besselfunktion erster Gattung nullter Ordnung, $\mathrm{k}_{\mathrm{f}}$ der Fermiwellenzahl, $\Phi$ einer frei anzupassenden Phase und $\kappa$ einem Dämpfungsfaktor. Abbildung 66 (a) zeigt die Ergebnisse der besten Anpassung mit den freien Parametern der Topographie (Spitzendurchmesser $\mathrm{D}_{\text {Spitze }}$, Tunnelabstand, $\lambda_{f}, \Phi$ sowie den Dämpfungskonstanten $\mathrm{k}_{\text {links }}$ und $\mathrm{k}_{\text {rechts }}$ ). Bei der Anpassung der Potentiometrie-Spur wurden ein lateraler Versatz zur „Oberflächentopographie“ und zwei unterschiedliche Dämpfungskonstanten als freie Parameter verwendet. 
Für die Anpassung von $\mu_{\mathrm{ec}, \text { Oberfläche }} \mathrm{J}_{0}=1$ gesetzt und die Dynamik auf Eins normiert. Die Anpassung des Tunnelabstands und des Spitzendurchmessers geschah ausschließlich an die Topographie-Daten.

a) Topographie

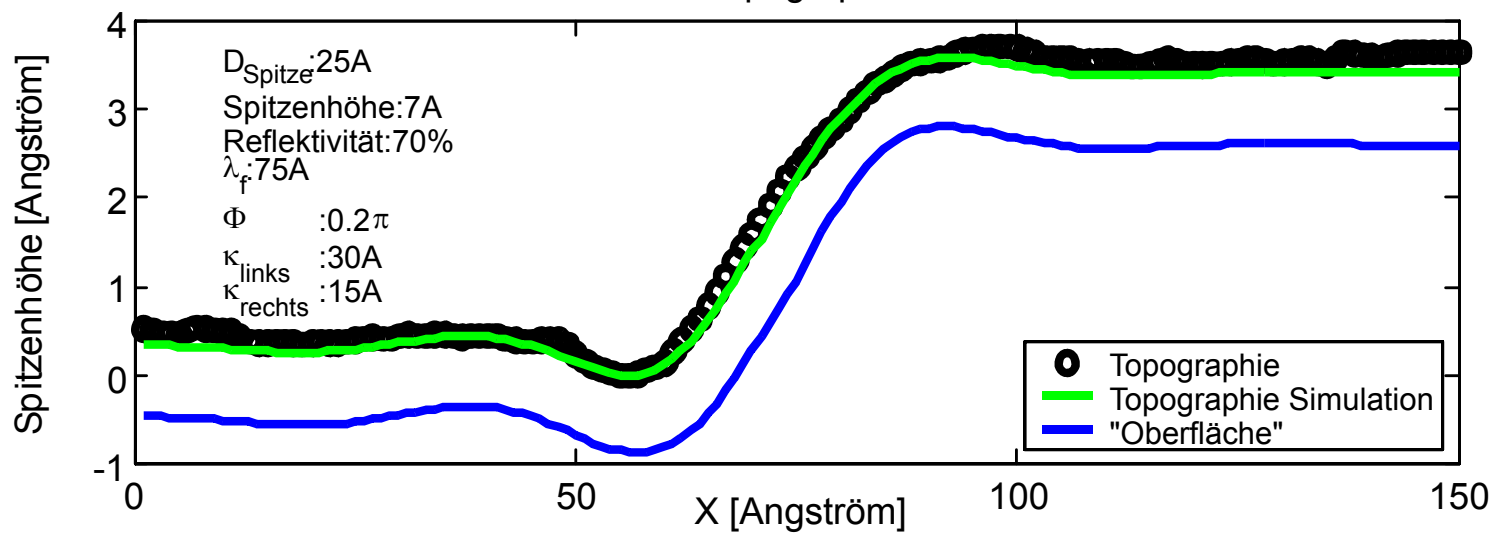

b)

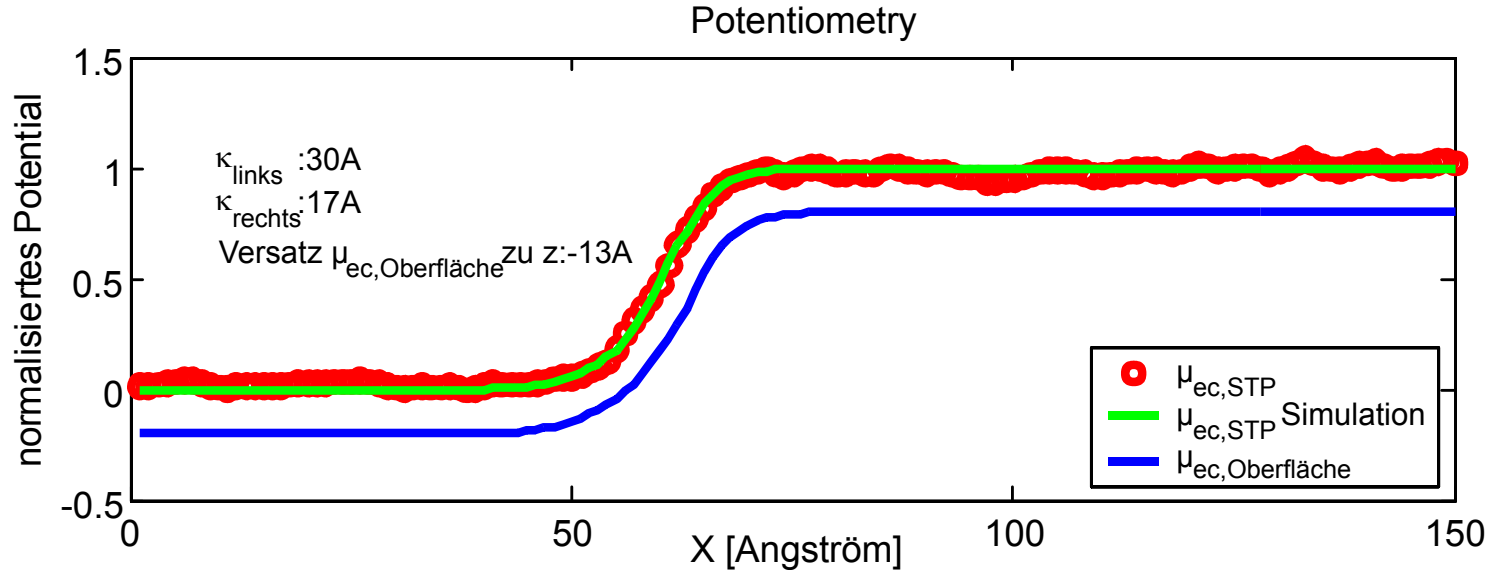

Abbildung 66: Ergebnisse der STP-Simulation mit bestmöglicher Übereinstimmung: (a) zeigt die Topographie mit ,friedelartig“ verschmierter Oberflächenstufe. Die simulierte Topographie-Spur (grün) passt sehr exakt zum gemessenen Verlauf (schwarze Punkte). In blau ist die verwendete Oberfläche skizziert. (b) illustriert den gemessenen Verlauf von $\mu_{e c, \text { STP (rote }}$

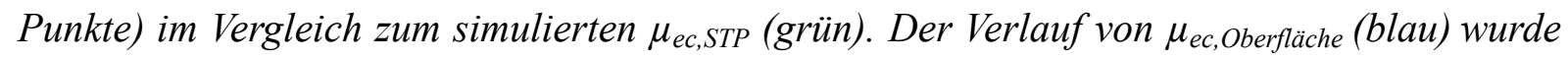
mit einer Exponentialfunktion verschmiert. Die experimentellen Daten sind Schnitte aus Abbildung 60, jeweils gemittelt über 20 A. Die Spitzenhöhe beginnt auf der unteren Terrasse bereits 8 A vor der physikalischen Position der Stufe zu variieren. Dies ist dadurch erklärbar, dass ein Anteil des Tunnelstroms von der oberen Terrasse getragen wird, obwohl sich die Spitze lateral noch 8 A davor befindet. 
Der so bestimmte Wert für den Spitzendurchmesser von $25 \AA$ ist konsistent mit SEMMessungen an einer Vielzahl von identisch präparierten Tunnelspitzen dieser Arbeitsgruppe [35, 123]. Dies wird durchaus als Validierung des Modells gewertet. Die TopographieOszillationen auf beiden Terrassenhälften und die Übergangsweite der Topographie entsprechen sehr exakt den experimentellen Befunden. Gleichermaßen entspricht der simulierte $\mu_{\mathrm{ec}, \mathrm{STP}}$-Verlauf sehr exakt den experimentellen Daten. Die im Rahmen der STPSimulation bestimmte Fermiwellenlänge von $\lambda_{f}=75 \AA$ erscheint konsistent zur STSBestimmung der Fermiwellenlänge mit $\lambda_{f}=68 \AA$ nach Kapitel 2.3.1.

Die für die weitere Diskussion wichtigen Ergebnisse aus dieser Modellierung sind zusammengefasst:

1. Topographie-Anpassung:

a. Eine bestmögliche Topographie Anpassung wird für einen Spitzendurchmesser von $25 \AA \AA$ und einem Tunnelabstand von $7 \AA$ erreicht.

b. Die Fermiwellenlänge wird $\mathrm{zu} \quad \lambda_{f}=75 \AA$ mit Dämpfungskonstanten von $30 \AA^{-1}$ auf der unteren und $15 \AA^{-1}$ auf der oberen Terrasse spezifiziert.

2. Potentiometrie-Anpassung

a. Der gemessene Verlauf von $\mu_{\mathrm{ec}, \mathrm{STP}}$ ist mit einer Stufenfunktion von $\mu_{\mathrm{ec}, \text { Oberfläche }}$ unvereinbar.

b. Die unabhängig angepassten Dämpfungskonstanten entsprechen den Dämpfungskonstanten der Topographie.

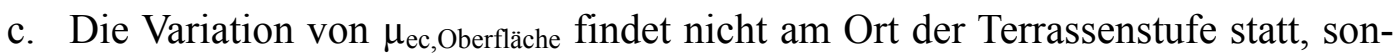
dern ist lateral um $13 \AA$ zur unteren Terrasse hin verschoben.

Dies sind interessante Ergebnisse und das ,ausgeschmierte“ elektrochemische Potential $\mu_{\mathrm{ec}, \text { Oberfläche }}$ steht im Widerspruch zu Dattas Vorhersage. Eine Interpretation wird im nächsten Kapitel (Kapitel 4.2) gegeben. Zunächst werden jedoch die Studien zur Parameterveränderung aufgezeigt, um das genannte Simulationsergebnis weiter zu verifizieren. Abbildung 67 fasst schematisch die Vielzahl von Modellstudien zusammen. (a) zeigt nochmals das bereits diskutierte, am besten passende Modell. 
b)
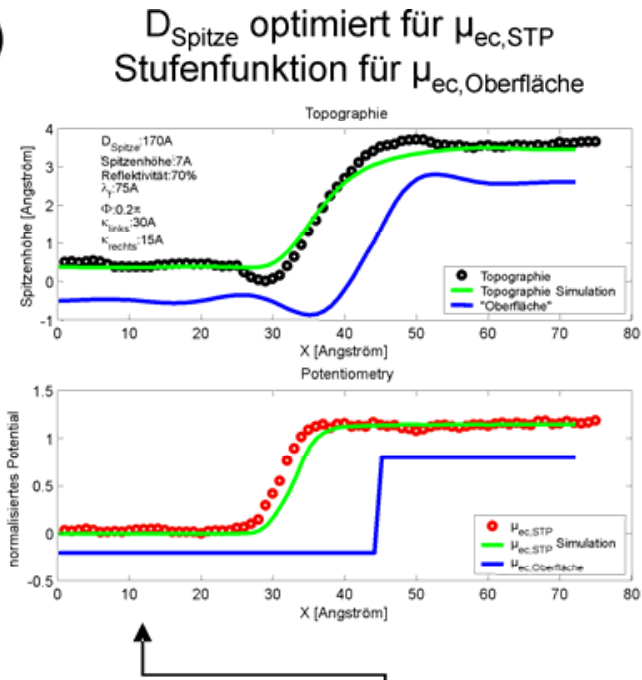

Unter Verwendung von $\mathrm{D}_{\text {Spize }}=170 \AA$ A wird die Variation von $\mu_{\text {ec,STP }}$ und auch dessen Versatz wiedergegeben. Die Topographiespur weicht jedoch stark von den experimentellen Daten ab.

Mit einer "artifiziellen“ Oberfläche und $D_{\text {Spitze }}=110$ Â wird zwar der Versatz reproduziert, jedoch ist die Variation in $\mu_{\text {ec STP }}$ schärfer als in den experimentellen Daten.

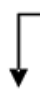

d) "artifizielle" Oberfläche um $\mathrm{D}_{\text {Spitze }}$ zu maximieren
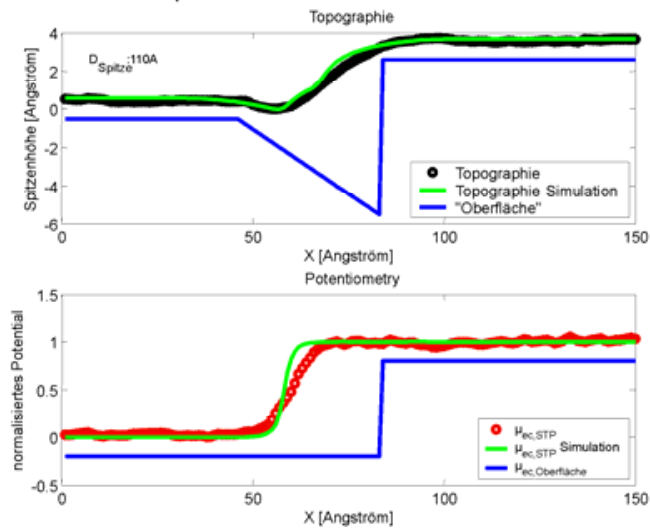

c) $D_{\text {Spitze }}$ optimiert für Topographie Stufenfunktion für $\mu_{\text {ec,Oberfläche }}$
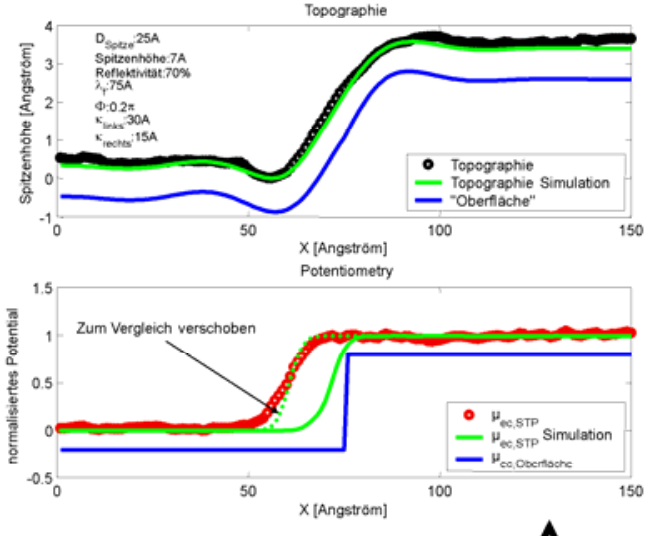

a) Beste Anpassung: $D_{\text {Spitze }}$ optimiert für Topographie $\mu_{\mathrm{ec}, \text { Oberfläche }}$ asymmetrisch verbreitert und versetzt
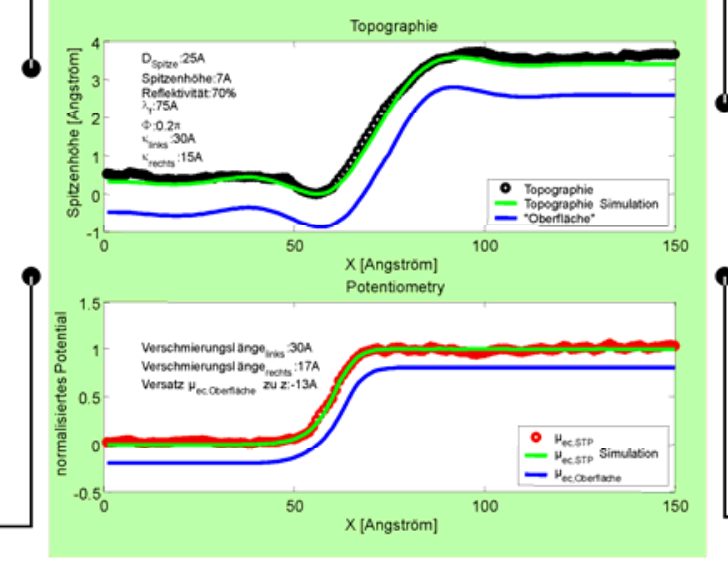

Eine symmetrische Verbreiterung von $\mu_{\text {ec Oberfläche liefert eine }}$ schlechtere Anpassung an die experimentellen Daten.

\section{$D_{\text {Spize }}$ optimiert für Topographie}

e) symmetrisch verbreitert und versetzt
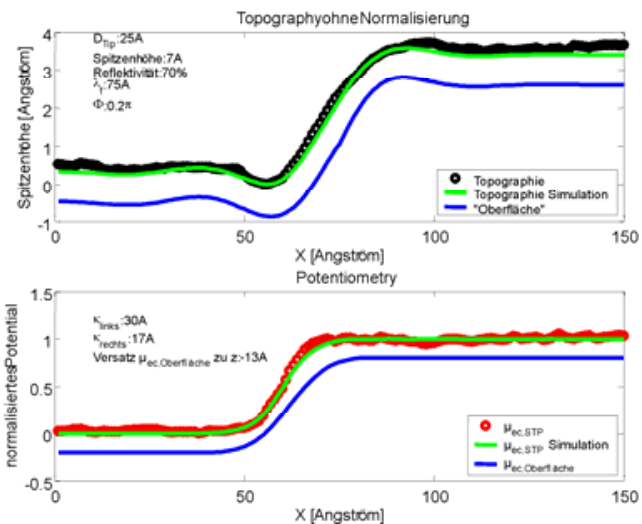

Abbildung 67: Flowchart zur Parametervariation und ihrer Auswirkungen. (a) präsentiert nochmals die Ergebnisse der besten Anpassung mit asymmetrisch verbreiteter und versetzter

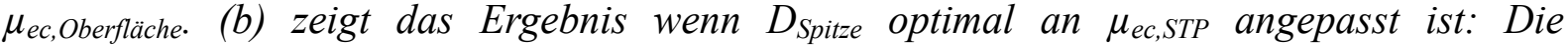
Topographie-Spur wird nicht wiedergegeben. (c) zeigt das Ergebnis bei Verwendung einer 


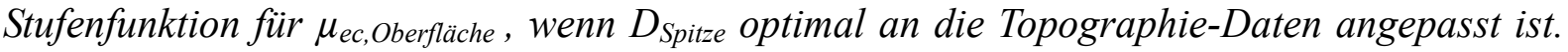
Der Übergang von $\mu_{e c, S T P}$ ist zu steil und der Versatz zur Topographie passt nicht. (d) illustriert eine ,, artifizielle“ Oberfläche mit einer 5 A tiefen Vertiefung vor der Stufe: Obwohl die Topographie-Spur in Maßen stimmt und auch der Versatz richtig wiedergegeben wird, ist der Übergang von $\mu_{e c, S T P} z$ steil. (e) illustriert das Ergebnis der besten Anpassung, jedoch

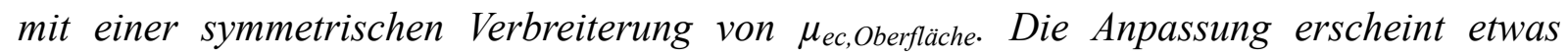
schlechter als mit einer asymmetrischen Verbreiterung. Die Abweichung ist allerdings minimal.

Diskussion $\mathrm{zu}$ (b): Möchte man gemäß Datta an einem abrupten Übergang von

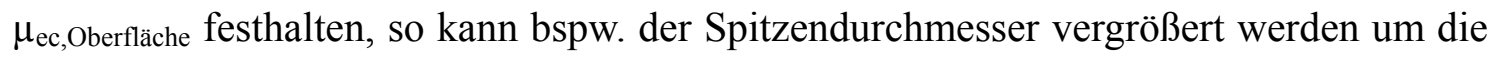
laterale Auflösung zu verringern. Es ist dadurch möglich, eine Anpassung an die expe-

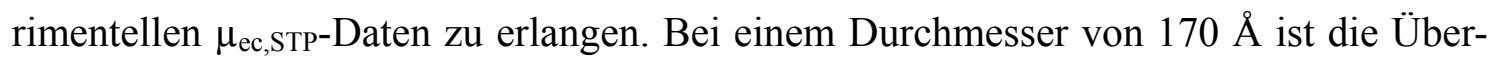
gangsweite in $\mu_{\mathrm{ec}, \mathrm{STP}}$ ähnlich den experimentellen Daten und auch der Versatz zur Topographie passt. Allerdings können die Friedel-Oszillationen zu beiden Seiten der Terrassenstufe in der Topographie mit ihrer lateralen Ausdehnung von weniger als $20 \AA$ nicht abgebildet werden, was im Widerspruch zu der experimentellen Beobachtung steht.

Diskussion zu (c): Optimiert man hingegen den Spitzendurchmesser für die Topographie, so ergibt sich ein eine zu kleine Übergangsweite in $\mu_{\text {ec,STP }}$ und auch der Versatz korrespondiert nicht zu den experimentellen Daten.

Diskussion zu (d): Die Topographie-Spur passt noch einigermaßen, allerdings weicht die berechnete Potentialspur noch substantiell von den experimentellen Daten ab. Selbst bei dieser drastischen Modellierung der LDOS kann der Spitzendurchmesser nicht weiter vergrößert werden, um die Übergangsweite im berechneten $\mu_{\mathrm{ec}, \mathrm{STP}}$ zu erhöhen. Andernfalls kann die Vertiefung vor der Terrassenstufe nicht mehr abgebildet werden. 
Diskussion zu (e): Verwendet man auf beiden Terrassenhälften dieselben Dämpfungskonstanten so ergibt sich eine etwas schlechtere Anpassung als bei (a). Aufgrund der geringen Abweichung ist eine Aussage über unterschiedliche Dämpfungskonstanten auf beiden Terrassenseiten allerdings schwierig.

Im Rahmen dieser Modellstudien sind die experimentellen Daten, insbesondere der Verlauf von $\mu_{\mathrm{ec}, \mathrm{STP}}$, nicht mit einer Stufenfunktion von $\mu_{\mathrm{ec}, \text { Oberfläche }} \mathrm{zu}$ beschreiben. Des Weiteren ist ein lateraler Versatz von $13 \AA$ zwischen der Barriere für den Ladungstransport und der Terrassenstufe notwendig. 


\subsection{Interpretation der Variation des elektrochemischen Poten- tials in der Umgebung von monoatomaren Substratstufen}

Im Folgenden wird der Versuch unternommen, den beobachteten und durch Simulationen

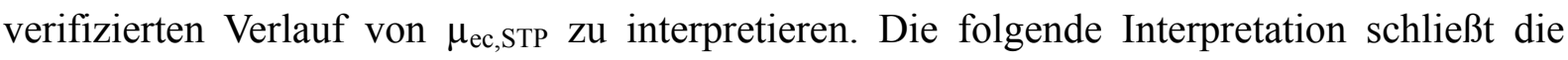
durch den Transport bedingte Nicht-Gleichgewichtsverteilung mit ein. Darüber hinaus wird versucht, von der idealisierten Vorstellung des rein diffusen oder rein ballistischen Transports Abstand zu nehmen und das System als mesoskopisch zu betrachten. Zunächst wird noch einmal die vorherrschende Lehrmeinung nach S. Datta illustriert, um sie anschließend dem eigenem Interpretationsansatz gegenüber zu stellen.

Abbildung 68 zeigt schematisch die einzelnen Prozesse während des ballistischen Ladungstransports über eine Barriere im Rahmen der Beschreibung von Datta [39]. Die Besetzung der $\mathrm{k}^{+}$- und $\mathrm{k}^{-}$-Zustände ist unterschiedlich, um einem Netto-Elektronentransport von links nach rechts $\mathrm{zu}$ entsprechen. Am Ort der Barriere werden Elektronen entweder mit 1-T reflektiert oder transmittierten mit der Wahrscheinlichkeit $\mathrm{T} z u(e)$. (a-h) illustrieren den Verlauf des quasi-chemischen Potentials in der Nähe der Tunnel-Barriere und -e $\Phi_{\text {elek. }}$ skizziert die entsprechende elektrostatische Energie. Die Kontaktbereiche sind nicht skizziert und es wird davon ausgegangen, dass in (a) und (h) die entsprechenden Besetzungen vorliegen. An der Barriere kommt es zur Ausbildung eines elektrostatischen Dipols und $\Phi_{\text {elek. }}$ variiert innerhalb der elektrostatischen Abschirmlänge. Vor der Barriere (d) ist die Elektronendichte erhöht und hinter der Barriere (e) erniedrigt. Nach Datta ist die Abschirmung am Ort der Barriere nicht durch den Transport beeinflusst und entspricht der elektrostatischen Abschirmung für die stromlose Situation, wie bspw. bei $\mathrm{T}=0$ [39]. Als Folge der ungestörten Abschirmung ist das elektrochemische Potential zu beiden Seiten der Barriere konstant und variiert abrupt am Ort der Barriere (siehe hierzu auch Abschnitt 1.2.2.2). Experimentell wird allerdings eine endliche Übergangsweite beobachtet, so dass diese Modellvorstellung erweitert werden muss. 


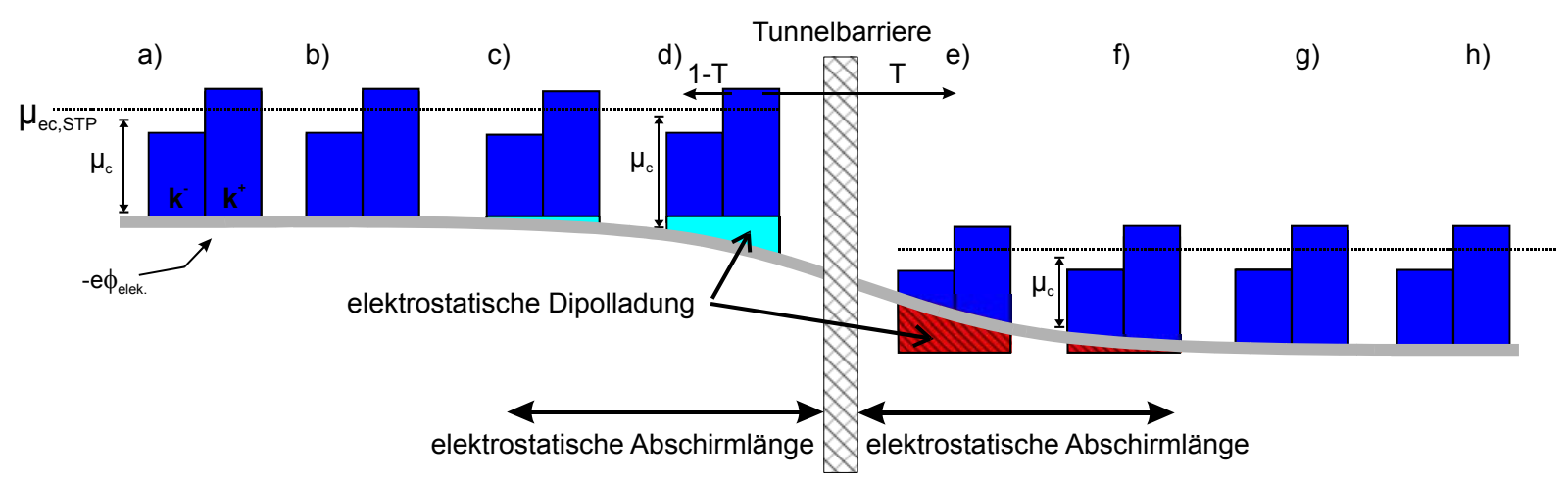

Abbildung 68: Schemaskizze zum Verlauf des elektrostatischen und der quasi-chemischen Potentiale innerhalb eines ballistischen Systems mit einer Tunnel-Barriere nach Datta [39]. Die Abschirmung entspricht dem Gleichgewichtsfall, dass heißt wie bei einer undurchlässigen Barriere. Als Konsequenz davon vermutet Datta, dass das elektrochemische Potential bis zur Barriere konstant ist und dann abrupt variiert.

Abbildung 69 zeigt einen Interpretationsansatz der die Nicht-Gleichgewichtsverteilung am Ort der Barriere und den nicht vollständig ballistischen Transport miteinschließt. Die relevanten Längenskalen liegen in einem Bereich, bei dem eine konzeptionelle Beschreibung vollständig quantenmechanisch unter Berücksichtigung der räumlich variierenden NichtGleichgewichtsverteilung stattfinden müsste. Dieser Zugang ist wenn überhaupt nur mit großem Aufwand verfügbar. Beim nun folgenden Interpretationsansatz kann dieser Sachverhalt deshalb nur sprachlich umschrieben werden. Häufig wird dabei im Rahmen des Welle-Teilchen Dualismus zwischen quantenmechanischen und semi-klassischen Konzepten gewechselt.

Durch den nicht vollständig ballistischen Ladungstransport ist die Kopplung innerhalb einer Mode verringert. Geschieht das Entvölkern von $\mathrm{k}^{+}$-Zuständen links der Barriere mit einer vergleichbaren Rate wie das Nachfüllen, so befinden sich die $\mathrm{k}^{+}$-Zustände vor der Barriere in einer starken Nicht-Gleichgewichtsverteilung. Die Besetzungen der quasi-chemischen Niveaus der $\mathrm{k}^{+}$-Zustände enthält in diesem Fall Löcher unter der Fermikante. Diese freien $\mathrm{k}^{+}-$ Zustände werden aus den übrigen $\mathrm{k}^{+}$-Zuständen links der Barriere nachgefüllt, so dass die Ungleichgewichtsverteilung sich gegen die eigentliche Propagationsrichtung der $\mathrm{k}^{+}$-Zustände nach links erstrecken könnte. Analoges gilt für die $\mathrm{k}^{-}$-Zustände rechts der Barriere. Schematisch zeigt (c-f) die daraus resultierende Besetzung für „heiße“ Löcher links der Barriere und „heiße“ Elektronen rechts der Barriere. Der Grundgedanke des Interpretationsansatzes ist, dass die über die Barriere transmittierenden Elektronen links der Barriere eine verminderte Ladungsdichte hinterlassen, die nicht unendlich schnell ausgeglichen werden 
kann. Dieselbe Argumentation ergibt einen Elektronenüberschuss rechts der Barriere. Im zeitlichen Mittel würde demnach eine Abweichung von der elektrostatischen DipolLadungsdichte resultieren. Die dynamisch veränderte elektrostatische Energie (-eФ) am Ort der Barriere zeigt Abbildung 69 (d, e) in gestrichelten Linien. Innerhalb einer Längenskala, die mit dem dynamischen Ausgleich von Ladungen verknüpft ist, weicht die elektrostatische Energie beim Netto-Stromtransport von der elektrostatischen Energie der Gleichgewichtsverteilung, d. h. ohne Stromtransport, ab. In diesem Sinn interpretieren wir die experimentell beobachtete Übergangsweite im elektrochemischen Potential als dynamisch veränderte Abschirmung als Folge des Transports am Ort die Barriere.

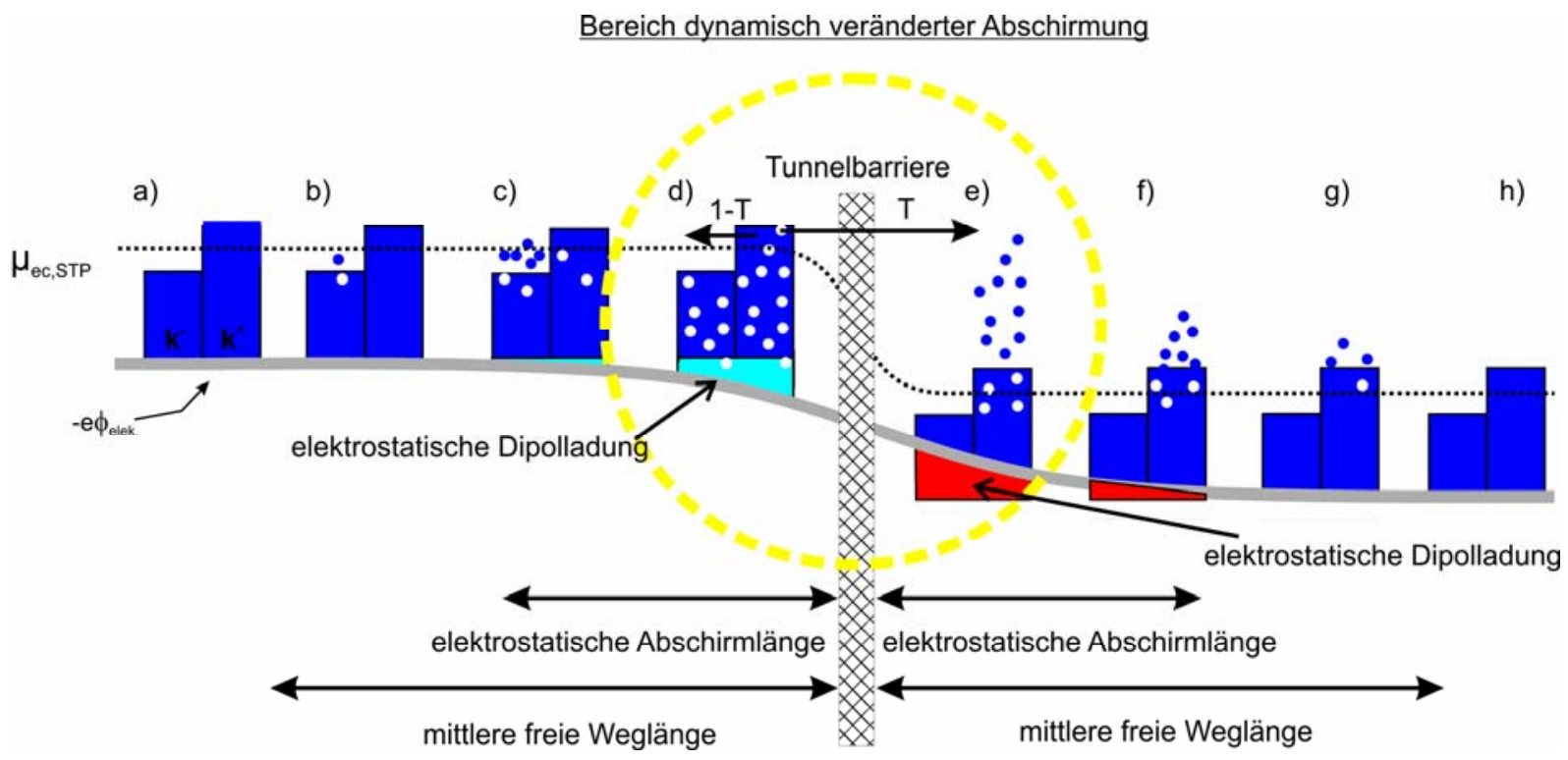

Abbildung 69: Schemaskizze zum Verlauf der quasi-Potentiale in einem mesoskopischen System mit einer Tunnel-Barriere als Erweiterung des Modells von Datta. Durch die Transmission über die Barriere werden heiße Löcher und Elektronen erzeugt, welche ihre Überschussenergie innerhalb der Energierelaxationslänge verlieren. Die elektrostatische Abschirmung wird durch den Transport über die Barriere behindert und als Konsequenz davon variiert das elektrochemische Potential monoton am Ort der Barriere innerhalb einer endlichen Übergangweite. 
Dieser Interpretation folgend, sollte eine dynamisch veränderte Abschirmung dort am größten sein, wo die lokale Zustandsdichte vermindert ist. Genau dies wird experimentell beobachtet. Abbildung 70 illustriert diesen Sachverhalt im Kontext der erarbeiten Interpretation. Das elektrochemische Potential (a) variiert maximal im ersten LDOS-Minimum der FriedelOszillation der Topographie (b). Die maximale Variation des elektrochemischen Potentials ist durch einen grauen Bereich angedeutet. Eine Seitenansicht der atomaren Struktur an der Stufe zeigt (c). Die Position der Stufe wurde hierfür von Hand an die Variation in der Topographie angepasst und um $8 \AA$ nach rechts verschoben, wie es die STP-Simulation spezifiziert. Die Topographie-Spur variiert demnach auf der unteren Terrasse bereits $8 \AA$ bevor der Spitzenmittelpunkt die physikalische Position der Stufe erreicht hat. Somit ist die beobachtete Variation in $\mu_{\mathrm{ec}, \mathrm{STP}}$ um ca. $\lambda_{\mathrm{F}} / 4=17 \AA$ der physikalischen Position der Stufe vorgelagert und die sich hieraus ergebende Differenz zwischen der lateralen Variation in $\mu_{\mathrm{ec}, \mathrm{STP}}$ und der Topographie entspricht dem experimentellen Befund von ca. $\Delta \mathrm{X}=10.8(5) \AA$. 
a)

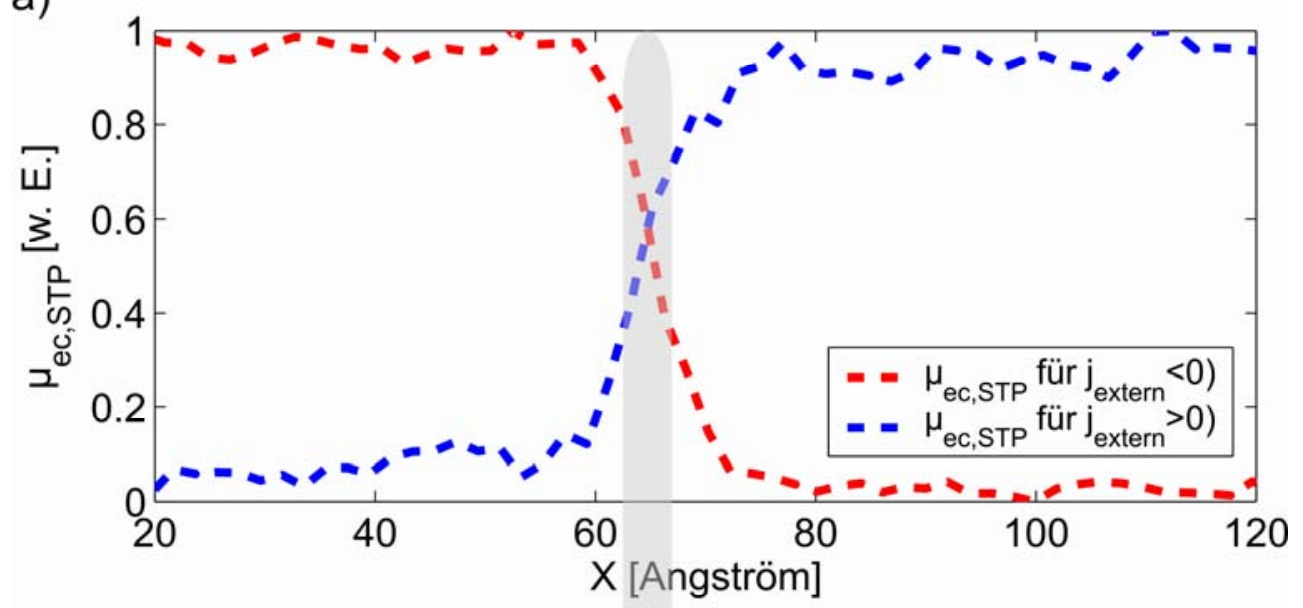

b)

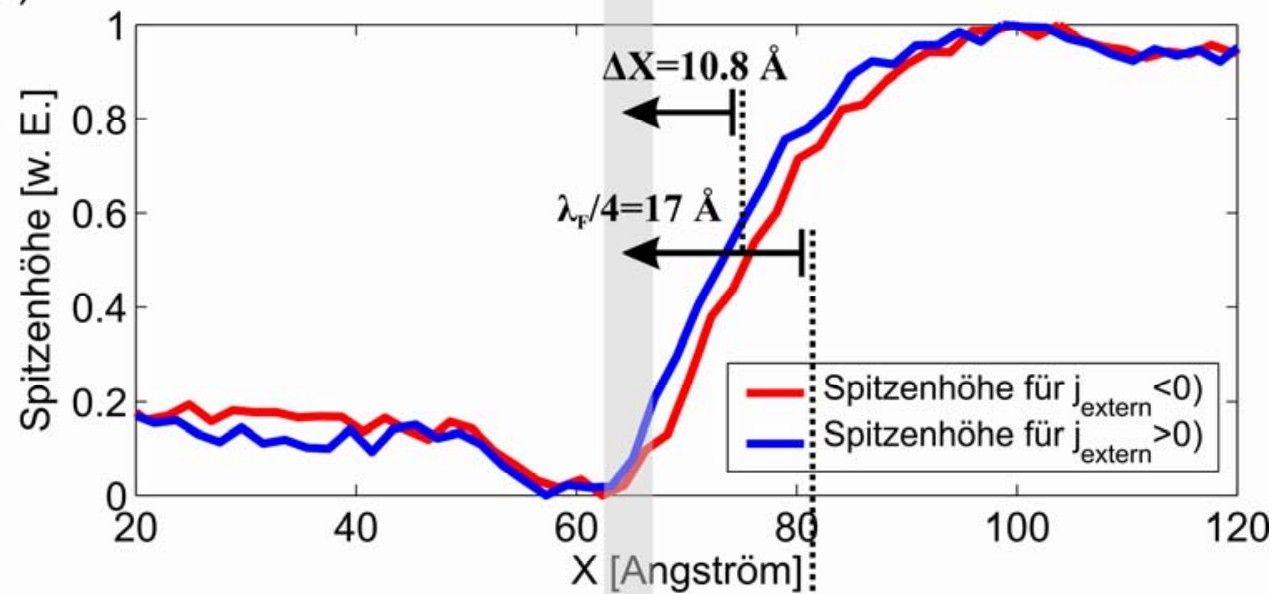

c)

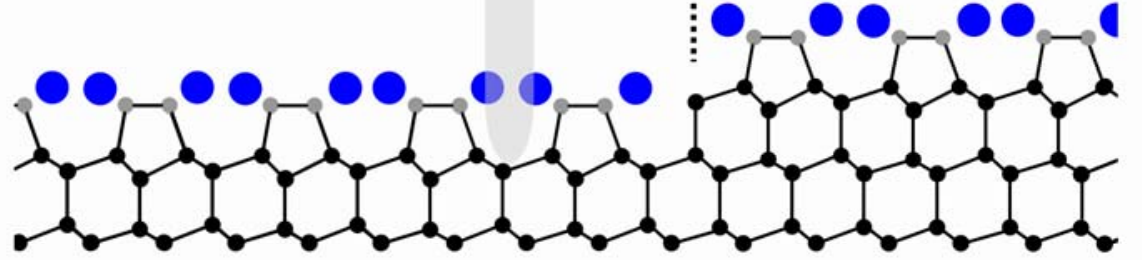

$$
\text { Ag-Atom oberstes Si-Atom } \quad \text { Si-Atom }
$$

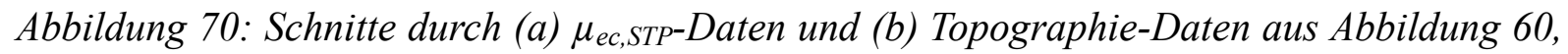
jeweils gemittelt über 20 A. Blau markiert Daten für $j_{\text {extern }}>0$ (Hinspur) und rot illustriert die Daten der Rückspur mit $j_{\text {extern }}<0$. Der Bereich maximaler Variation in $\mu_{e c, \text { STP }}$ liegt im LDOSMinimum der Friedel-Oszillation und ist der Stufe um ca. $\lambda_{F} / 4$ vorgelagert (grauer Bereich). (c) illustriert schematisch die Seitenansicht der atomaren Struktur einer monoatomaren Stufe in der (110)-Ebene gemäß Ref. [73, 102]. Die Stufe verläuft entlang der [110]-Richtung. Die „Stufenposition“ wurde von Hand an die gemessene Variation der Topographie angepasst und 8 A weiter nach rechts verlagert, wie es die STP-Simulation gemäß Abbildung 66 anzeigt. 
Theoretisch sollte ein lateraler Versatz von $\Delta X=9 \AA$ auftreten, was jedoch als gute Übereinstimmung angesehen wird. Mögliche Fehlerquellen sind die genaue Phasenlage der FriedelOszillation sowie die genaue elektronische Struktur an der Stufe.

Eine Erklärung warum der Versatz des elektrochemischen Potentials zugunsten der unteren Terrasse erfolgt, kann im Rahmen dieser Arbeit nicht abschließend gegeben werden. Es ist jedoch zu bedenken, dass die Realstruktur an der Stufe nicht symmetrisch ist. Der auf der unteren Terrasse lokalisierte Oberflächenzustand wird durch eine Monolage Silizium begrenzt, wohingegen der obere Oberflächenzustand lateral durch „Vakuum“ begrenzt ist. Die Defektzustände auf der oberen und unteren Terrasse sind daher unterschiedlich, was im Rahmen von „Tight-Binding“ Berechnung bestätigt wird [124].

Die hier erstmalig aufgestellte Interpretation einer endlichen, nicht abbildungsbedingten Übergangsweite von $\mu_{\mathrm{ec}, \mathrm{STP}}$, als dynamisch veränderte Abschirmung in Folge des Transports über die Barriere, ist somit konsistent mit den aus STS-Messungen gewonnenen elektronischen Eigenschaften. Eine dominierende Beeinflussung von $\mu_{\mathrm{ec}, \mathrm{STP}}$ durch die energetische Relaxation der angeregten Ladungsträger am Ort der Barriere kann ausgeschlossen werden, da keine Variationen innerhalb der Energierelaxationslänge beobachtet worden sind. ${ }^{29}$

${ }^{29}$ Elektronen, die die Barriere elastisch durchquert haben, sind ,heiße“ Elektronen gemäß der quasi-Gleichgewichtsbesetzung fern der Barriere. Innerhalb der Energierelaxationslänge werden diese Elektronen in die thermische Besetzungsfunktion zum jeweiligen Zustand einsortiert. Eine Thermalisierung kann zunächst über Elektron-Elektron-Wechselwirkung erfolgen, allerdings wird der Energiebetrag letztendlich über Elektron-Phonon-Stöße an das Gitter übergeben. Die Energierelaxationslänge ist für die $\operatorname{Si}(111)(\sqrt{ } 3 \mathrm{x} \sqrt{ } 3)-A g$ Rekonstruktion unbekannt. Sie kann allerdings über die mittlere freie Weglänge im Rahmen der DrudeSommerfeld-Beschreibung abgeschätzt werden. Mit $\mathrm{E}_{\mathrm{f}}=250 \mathrm{meV}$ und $\mathrm{m}^{*}=0.13$ beträgt die mittlere freie Weglänge bei einer 2DEG-Leitfähigkeit von $3080 \mu \mathrm{S} / \square$ (Werte siehe Kapitel 3.2.2.2) etwa $70 \mathrm{~nm}$. Die beobachtete Übergangsweite von $\mu_{\mathrm{ec}, \mathrm{STP}}$ von $\mathrm{L}_{\Delta \mu}=5.6(4) \AA$ ist um den Faktor $\sim 100$ kleiner, so dass die Energierelaxation nur eine untergeordnete Rolle spielen kann. 


\section{$5 \quad$ Zusammenfassung}

In dieser Arbeit wird der Ladungstransport durch das zweidimensionale Elektronengas der $\operatorname{Si}(111)(\sqrt{3} \mathrm{x} \sqrt{3})$-Ag Rekonstruktion als prototypisches Modellsystem für elektrische Leiter mit Hilfe der Raster-Tunnel-Potentiometrie untersucht. Hierzu wurde ein zweidimensionales Elektronengas auf der Oberfläche eines Si(111)-Wafers im UHV durch Silber-Adsorption geschaffen und in situ für Transportuntersuchungen elektrisch kontaktiert. Vorab wurde die atomare Struktur aufgeklärt, wonach die IET-Struktur auch bei Raumtemperatur die stabile atomare Konfiguration dieser Rekonstruktion ist. Die mikroskopischen Prozesse, welche sich zum makroskopischen Widerstand aufaddieren, wurden im Ortsraum einzeln beobachtet und analysiert. Neben diffusen Streuprozessen durch Elektron-Phonon- und Elektron-ElektronStreuung wurden Stufenkanten des Substrats und zwei Arten von Domänengrenzen als lokalisierte Streuprozesse identifiziert.

Die detaillierte Analyse der Variation des elektrochemischen Potentials wurde an monoatomaren Stufenkanten vorgenommen. Ein Ergebnis dieser Analysen ist, dass die Variation des elektrochemischen Potentials eine lineare Antwort der externen Stromdichte ist. Der Transmissionskoeffizient ist demzufolge im relevanten Energieintervall für Transportexperimente energieunabhängig.

Der räumliche Verlauf des elektrochemischen Potentials am Ort einer monoatomaren Stufe wurde mit einer Auflösung von bis zu $2 \AA$ analysiert. Im Zusammenspiel mit einer STPSimulation konnte verifiziert werden, dass die beobachteten Variationen keine abbildungsbedingten Artefakte beinhalten. Das elektrochemische Potential variiert demnach monoton von der unteren zur oberen Terrasse innerhalb einer Übergangsweite von 5.6(4) $\AA$ und ist der physikalischen Position der Terrassenstufe lateral um ca. $17 \AA$ entsprechend $\lambda_{\mathrm{F}} / 4$ vorgelagert. Die experimentell beobachtete Übergangsweite im elektrochemischen Potential wird einer dynamisch verminderten Abschirmung als Folge des Transports am Ort der Barriere zugewiesen. Ladungsträger, die zur elektrostatischen Abschirmung des Potentialunterschieds am Ort der Barriere benötigt werden, transmittieren im Rahmen dieser Interpretation durch die Barriere, woraus eine unzureichende elektrostatische Abschirmung resultiert. Diese Interpretation entspricht einer konzeptionellen Erweiterung der Ladungstransportmodellierung nach S. Datta [39]. Es bleibt abzuwarten, ob sie in zukünftigen theoretischen Modellen aufgegriffen wird. 
Die durchgeführten Experimente analysieren darüber hinaus den Übergang von den elementaren mikroskopischen Prozessen zum makroskopischen Widerstand. Mit Hilfe einer Transportmodellierung konnte der räumliche Verlauf des elektrochemischen Potentials in einem realen System mit lokalisierten Defekten untersucht werden. Voraussetzung hierfür war eine strukturelle Charakterisierung durch STM-Topographien. Dies ermöglichte es erstmalig, die spezifischen Leitfähigkeiten für jeden physikalischen Prozess auf Basis mikroskopischer Messungen zu ermitteln. Ein zentrales Resultat dieser Analysen ist, dass die intrinsische Leitfähigkeit des zweidimensionalen Elektronengases der $\operatorname{Si}(111)(\sqrt{ } 3 \mathrm{x} \sqrt{ } 3)$-Ag Rekonstruktion bei Raumtemperatur und einer Besetzung des $S_{1}$-Oberflächenzustands von $E_{f}=250 \mathrm{meV}$ $3080 \pm 1100 \mu \mathrm{S} / \square$ beträgt. Die Transmission über monoatomare Terrassenstufen von $30 \%$ entspricht im Rahmen dieser Beschreibung einer Stufenleitfähigkeit von $\sigma_{\text {Step }}=5000 \Omega^{-1} \mathrm{~m}^{-1}$.

Im Zusammenspiel mit den strukturellen Informationen aus großskaligen STM-Topographien konnte auf Basis dieser mikroskopischen Größen der Ladungstransport bis zur makroskopischen Skala modelliert werden. Ein Resultat dieser Analysen ist, dass die Stromdichte durch die $\operatorname{Si}(111)(\sqrt{3} \mathrm{x} \sqrt{3})$-Ag Rekonstruktion im Allgemeinen stark inhomogen ist. Der Ladungstransport wird auf mesoskopischer bis makroskopischer Skala durch ein Perkolationsnetzwerk beeinflusst, dass sich während des Wachstums der $\operatorname{Si}(111)(\sqrt{3} x \sqrt{3})-A g$ Rekonstruktion selbstorganisiert formiert. Eine Vielzahl unterschiedlicher Literaturwerte zur Leitfähigkeit durch die $\operatorname{Si}(111)(\sqrt{3} \times \sqrt{ } 3)$-Ag Rekonstruktion aus Vierpunktmessungen kann durch die Rolle des natürlichen Perkolationsnetzwerks zusammenhängend erklärt werden. Die eigenen Resultate spezifizieren hierzu eine konstante Leitfähigkeit durch das natürliche Perkolationsnetzwerk von $\sim 800 \mu \mathrm{S} / \square$ oberhalb von $500 \mathrm{~nm}$ quadratischer Sheet-Größe.

Darüber hinaus wurden STP-Messungen auf der Si(111)( $\sqrt{3} \mathrm{x} \sqrt{ } 3)$-Ag Rekonstruktion auf stark gestuften Substraten analysiert. Eine Modellierung des Ladungstransports konnte auch auf dieses System erfolgreich angewandt werden. Die Analysen zeigen jedoch eindrucksvoll, dass eine Beschreibung des Ladungstransports mit konstanten mikroskopischen Größen in beliebigen Strukturen erweitert werden muss. So ist die quantenmechanische Transmission über eine Potentialbarriere eine winkelabhängige Funktion und die Streuung an lateralen Grenzflächen (Analogon zu Fuchs-Sondheimer) kann die effektive Leitfähigkeit des 2DEG auf einer Terrasse vermindern. Für eine mittlere Stromrichtung parallel zu Substratstufen konnte eine Verringerung der Stufentransmission um den Faktor 10 nachgewiesen werden. Die experimentell bestimmte Stufentransmission des natürlichen Perkolationsnetzwerks wird daher als Mittelwert der Transmissionsfunktion über alle Einfallswinkel interpretiert. 


\section{Anhang A: Konsistenzuntersuchungen zum Einfluss zusätzlicher Stützstellen}

\section{Normalverteiltes Rauschen}

Die gemessenen $\mu_{\mathrm{ec}, \mathrm{STP}}(\mathrm{x}, \mathrm{y})$-Daten sind leider häufig durch niederfrequente Störsignale (Drift) überlagert. Ein Großteil davon kann zwar durch individuelle Nachbearbeitung in Form von Linien- oder Ebenenabzug herausgefiltert werden. Allerdings wird hierdurch aber die quantitative Anpassung der spezifischen Widerstände beeinflusst. Um den Einfluss dieser Störsignale zu minimieren und um eine Aussage zur Signifikanz und Varianz der spezifischen Widerstände machen zu können, werden wenige zusätzliche Stützstellen in der Bildmitte verwendet. Sie ermöglichen, dass die Potentiallandschaft in der Nähe dieser Stützstellen wieder an das gemessene Potential angepasst wird. Der Einfluss dieser zusätzlichen Stützstellen sowie der Messdatenbearbeitung auf die Ergebnisse der Widerstandsanpassung soll im Folgenden dargestellt werden.

\section{Messdaten mit normalverteiltem Rauschen}

Um herauszufinden, welche Auswirkungen die o. g. zusätzlicher Stützstellen auf die Anpassung der Leitfähigkeiten haben, wurde aus gemessenen Topographie-Daten eine Leitfähigkeitsmatrix gemäß den Ergebnissen aus Kapitel 3.2.2.2 generiert $\left(\sigma_{2 D E G}=3080 \mu \mathrm{S} / \square, \sigma_{\text {Stufe }}=5000 \Omega^{-1} m^{-1}\right)$. Abbildung 71 (b) zeigt die hieraus berechnete Potentiallandschaft mit der Randbedingung, dass das Potential an den Rändern einem linearen Anstieg in x-Richtung folgt und in y-Richtung konstant ist. Ein normalverteiltes Rauschen mit einer Standardabweichung $\sigma_{\text {std }}$ von $10 \%$ der Dynamik der errechneten Potentiallandschaft zeigt (c) und die hieraus generierten „Messdaten“, welche als sauber definierte Testpotentiale für die weitere Analyse fungieren, zeigt (d). Sie entsprechen einer STP-Messung mit überlagertem normalverteiltem Rauschen. Zu beachten ist, dass das Signal zu Rauschverhältnis schlechter als in wirklichen Messdaten ist. 
Leitfähigkeitsmatrix

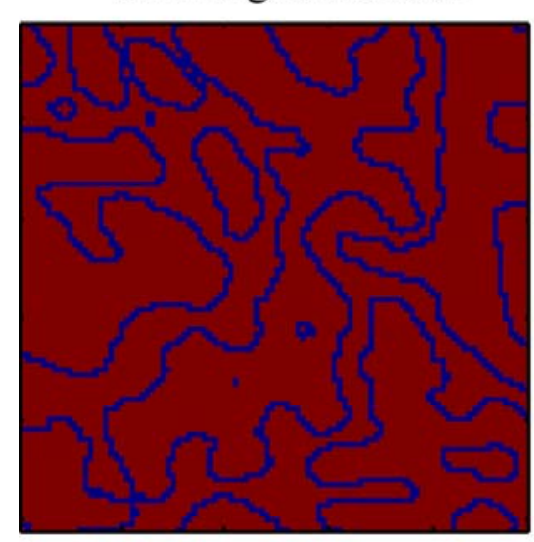

a)

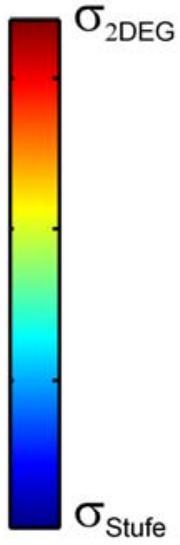

normalverteiltes Rauschen

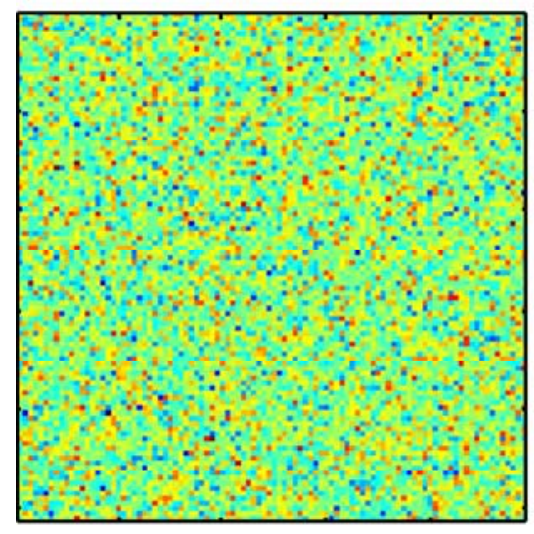

c)
Berechnete Potentiallandschaft

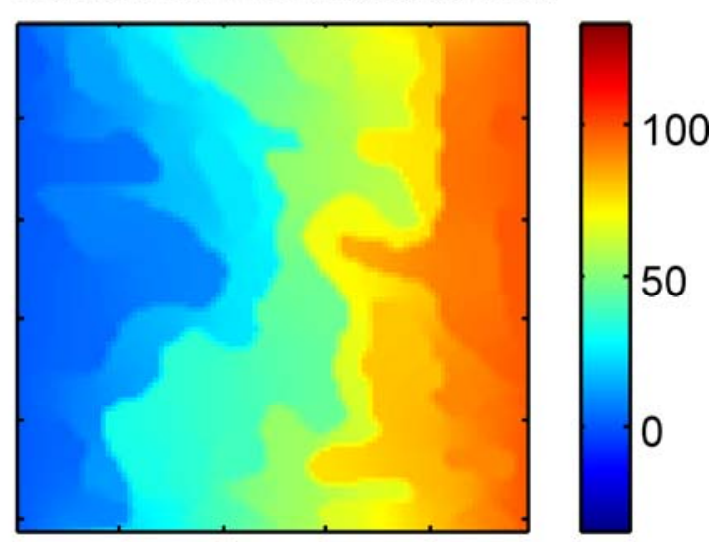

b)

Berechnete Potentiallandschaft + Rauschen
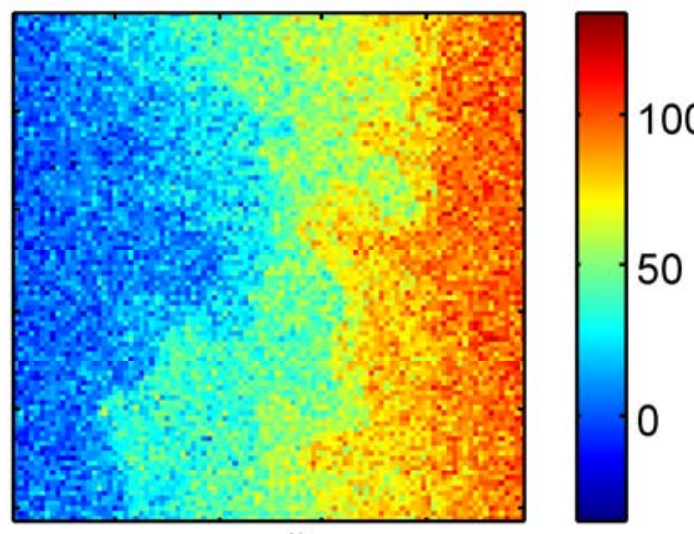

d)

Abbildung 71: (a) zeigt die verwendete Leitfähigkeitsmatrix gewonnen aus einer STMTopographie. Die Farbskala entspricht der lokalen Leitfähigkeit. (b) illustriert die hieraus berechnete Potentiallandschaft mit der Randbedingung eines linear in x-Richtung ansteigenden Potentials an den Rändern. Die Farbskala zeigt das lokale Potential. (c) zeigt normalverteiltes Rauschen mit einer Standardabweichung $\sigma_{\text {std }}$ von $10 \%$ der Dynamik aus (b). (d) illustriert die Summe von (b) und (c). Diese Potentiallandschaft wird im Folgenden als Test-Messdatensatz für $\mu_{e c, S T P}(x, y)$ verwendet.

Abbildung 72 zeigt die Ergebnisse der Leitfähigkeitsanpassung wenn die verrauschten Potentialwerte als „Messdaten“ verwendet werden. Die berechneten Werte schwanken um den Medianwert von $3270 \mu \mathrm{S} / \square$. Die Standardabweichung beträgt $600 \mu \mathrm{S} / \square$, so dass innerhalb des Fehlers die eingesetzte 2DEG-Leitfähigkeit von $3080 \mu \mathrm{S} / \square$ gut angepasst wird. Die bestimmten Werte sind nahezu normalverteilt und spiegeln damit das Rauschen der Messdaten wieder. Es erscheint auf den ersten Blick verwunderlich, dass der Fehler in der Bestimmung der Leitfähigkeit größer erscheint, als dass Rauschen in den Messdaten dies 
erwarten lässt. Begründen lässt sich dies damit, dass die Stützstellen ebenfalls mit Rauschen behaftet sind. Eine Stützstelle am Bildrand, welche eine starke Abweichung von seinem eigentlichen Wert hat, kann abhängig von der Struktur eine große Potentialfläche definieren. Dies kann bspw. geschehen, wenn ein enger Steg am Rand liegt und zu einem großen geschlossenem Bereich verbunden ist. Das Rauschen der Randpixel die als Randbedingungen dienen, verschiebt dann das Potential des ganzen geschlossenen Bereichs. Die Leitfähigkeit der begrenzenden Kanten wird in einem solchen Fall gegenüber dem wahren Wert verringert/vergrößert, um das Potential des geschlossenen und durch die „falschen“ Randbedingungen definierten Bereichs anzupassen.

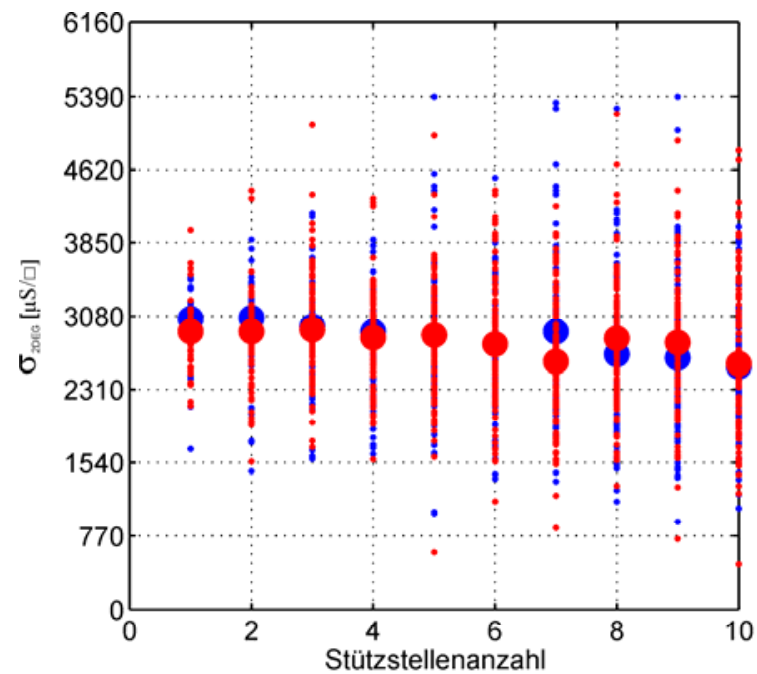

a)

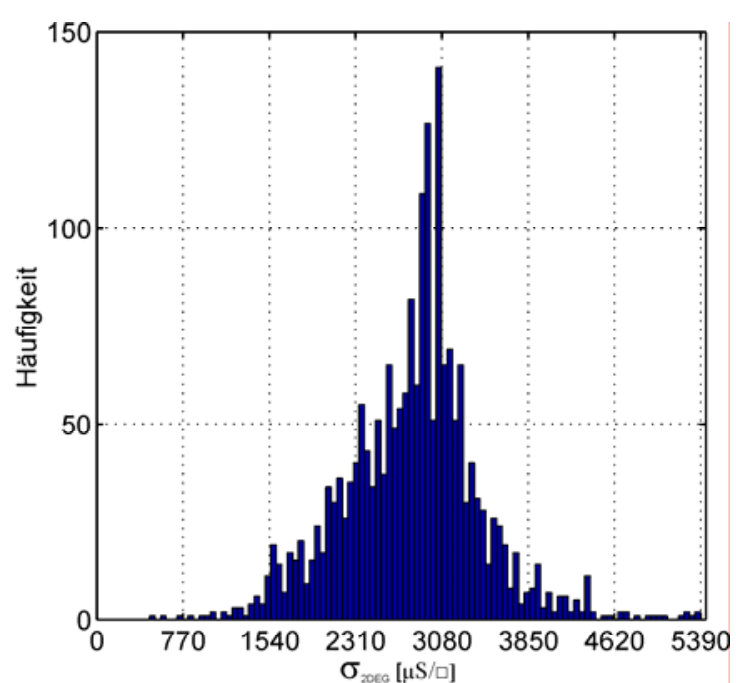

b)

Abbildung 72: Ergebnisse der Anpassung für eine Testpotentiallandschaft mit normalverteiltem Rauschen (blau) nach Abbildung 71. In Rot sind die Ergebnisse für das invertierte Rauschen dargestellt. (a) Die berechnete 2DEG-Leitfähigkeit schwankt um den Medianwert von $3270 \pm 600 \mu \mathrm{S} / \square$. Die Varianz nimmt mit der Stützstellenanzahl zu und die Signifikanz der Berechnung sinkt, da bei vielen Stützstellen viele Potentialgebiete stark durch die Stützstellen beeinflusst werden. Dementsprechend nimmt die Schwankungsbreite mit der Stützstellenanzahl zu. (b) Histogramm der 2DEG-Leitfähigkeit aller berechneten Konfigurationen aus (a). Die Werte sind nahezu normalverteilt und spiegeln damit das normalverteilte Rauschen der Testpotentiallandschaft wieder.

Abbildung 73 verdeutlicht dies. Wird das Potential am rechten Randpixel gegenüber dem ungestörtem Wert zu hoch gemessen, so wird die 2DEG-Leitfähigkeit um die Summe der Abweichungsquadrate zu minimieren, erhöht. Wird das Potential zu klein gemessen, wird die 2DEG-Leitfähigkeit verringert. Abbildung 72 zeigt ebenfalls eine Anpassung mit invertiertem 
Rauschen. Wie zu erwarten ist, werden beim invertierten Rauschen aufgrund dieser Tatsache leicht andere Werte spezifiziert.

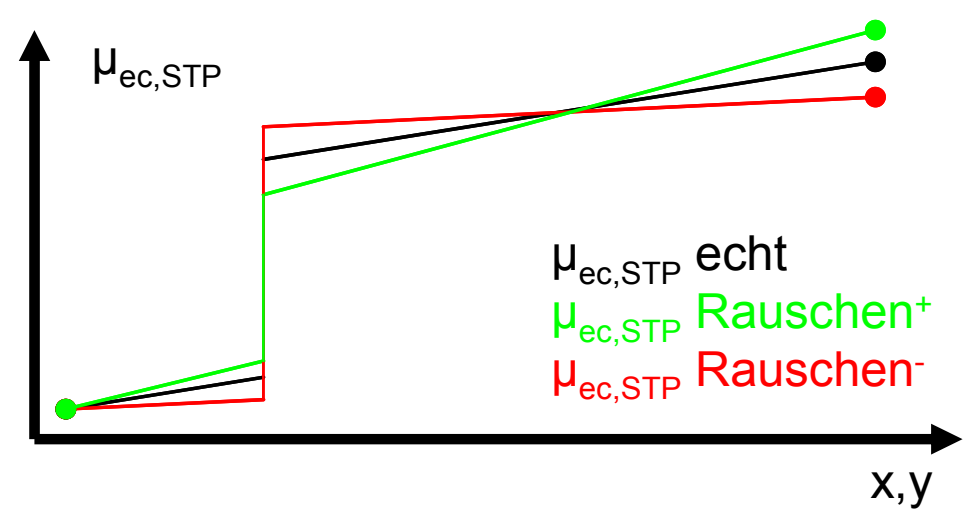

Abbildung 73: Schemaskizze zur Stufenleitfähigkeit bei verrauschten Randpixel. Die schwarze Kurve illustriert den ungestörten Potentialverlauf. Wird der rechte Randpixel durch Rauschen falsch gemessen, so ergeben sich andere Stufenleitfähigkeiten um die Summe der Abweichungsquadrate der Potentialverläufe zu minimieren.

In einer Übersicht zeigt Abbildung 74 die Testpotentiallandschaft zusammen mit der berechneten Potentiallandschaft und Abbildung 74(c) die Differenz der Testpotentiallandschaft von den errechneten Werten.
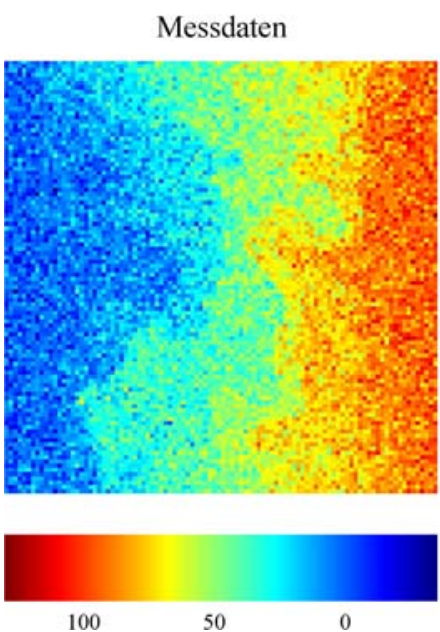

Simulation
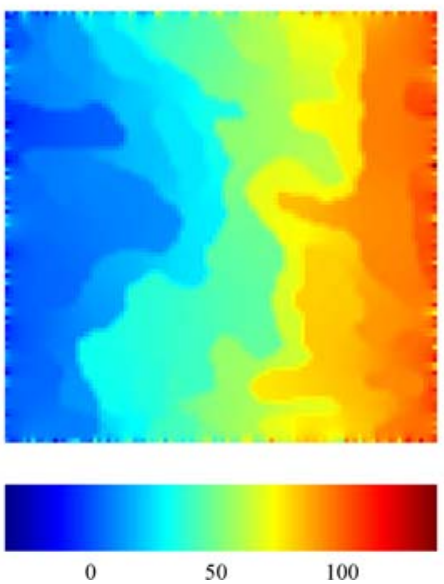

Differenz
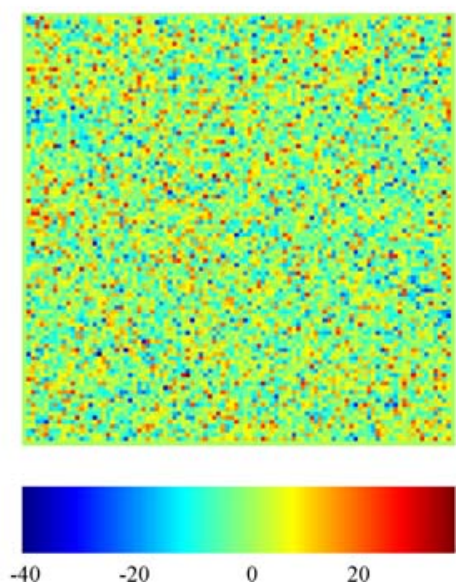

Abbildung 74: Vergleich der Testpotentiallandschaft mit normalverteiltem Rauschen (a) mit der berechneten Potentiallandschaft (b). Als Widerstandsverhältnis wurde der Mittelwert von $2790 \mu \mathrm{S} / \square$ verwendet. (c) zeigt die Differenz der Testpotentiallandschaft mit der errechneten Potentiallandschaft. 
Bis auf das normalverteilte Rauschen sind im Rahmen der darstellbaren Farbdynamik keine Abweichungen von der Testpotentiallandschaft zu erkennen. Als wichtiges Ergebnis kann demnach festgehalten werden, dass das Einführen zusätzlicher Stützstellen keinen verfälschenden Einfluss auf die Bestimmung der Leitfähigkeiten hat, sofern den $\mu_{\mathrm{ec}, \mathrm{STP}}(\mathrm{x}, \mathrm{y})$-Messdaten ein normalverteiltes Rauschen überlagert ist. Es sollte jedoch erwähnt werden, dass bei der Verwendung zusätzlicher Stützstellen die „Fläche“, bei der sich das Potential selbstkonsistent einstellt, verkleinert. Folglich sinkt die physikalische Aussagekraft der errechneten Leitfähigkeiten mit steigender Stützstellenanzahl. Gleichzeitig nimmt die Varianz der errechneten Leitfähigkeiten nach Abbildung 72(a) zu. Vor diesem Hintergrund sollte die Anzahl zusätzlicher Stützstellen möglichst minimal gehalten werden.

\section{Bearbeitung der Messdaten durch Linienabzug}

Häufig ist den Messdaten ein Störsignal entlang der langsamen Rasterrichtung überlagert. Solche Störeinflüsse werden meist durch Abzug des Mittelwerts (Linienabzug) einer jeden Rasterzeile minimiert. Abbildung 75(a) zeigt das bereits verwendete Testpotential aus dem vorherigen Abschnitt. Abbildung 75(c) illustriert das neue Testpotential für diesen Abschnitt nach Abzug des Mittelwerts einer jeden Zeile.

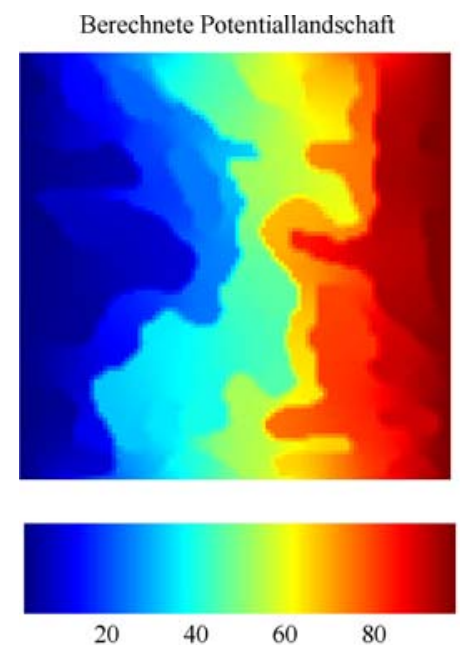

a)
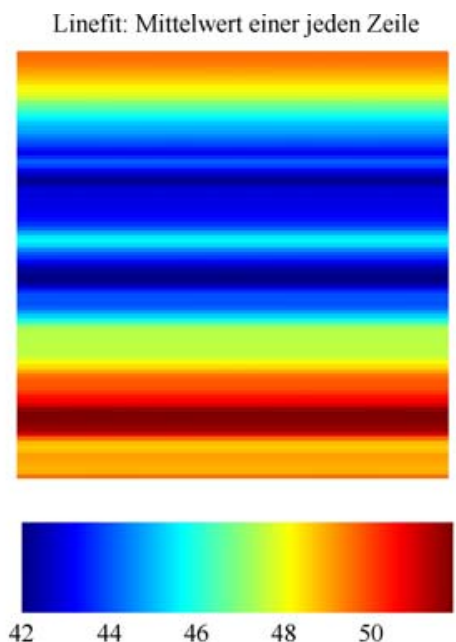

b)
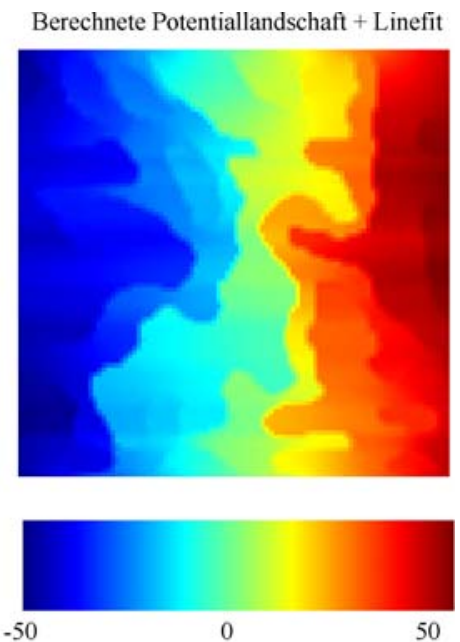

c)

Abbildung 75: Dem bereits verwendetem Testpotential aus dem vorherigen Abschnitt (a) wird der Mittelwert einer jeden Rasterzeile (b) abgezogen. Das neue Testpotential (c) zeigt dadurch linienartige Störungen, welche häufig bei der Bildbearbeitung auftreten. 
Die Ergebnisse mit diesem Testpotential, welches durch Linienabzug verfremdet wurde, zeigt Abbildung 76. Hier zeigt sich die Notwendigkeit zusätzliche Stützstellen zu verwenden. Je nachdem ob der Mittelwert einer jeden Zeile abgezogen (blau) oder addiert (rot) wurde, ergeben sich unterschiedliche Medianwerte der 2DEG-Leitfähigkeiten von $3660 \pm 1090 \mu \mathrm{S} / \square$ (blau) bzw. $2460 \pm 380 \mu \mathrm{S} / \square$ (rot), angegeben als Medianwert einer jeden Klasse. Zu beachten ist, dass sich sowohl bei der Addition als auch bei der Subtraktion die 2DEG-Leitfähigkeiten der eingesetzten 2DEG-Leitfähigkeit von $3080 \mu \mathrm{S} / \square$ annähern. Bei 50 zusätzlichen Stützstellen, dies bedeutet $5 \%$ der Messpunkte werden als zusätzliche Stützstellen verwendet, wird ein Leitfähigkeitsverhältnis von $3260 \pm 525 \mu \mathrm{S} / \square$ (blau) bzw. $2480 \pm 400$ $\mu \mathrm{S} / \square$ (rot) gefunden.

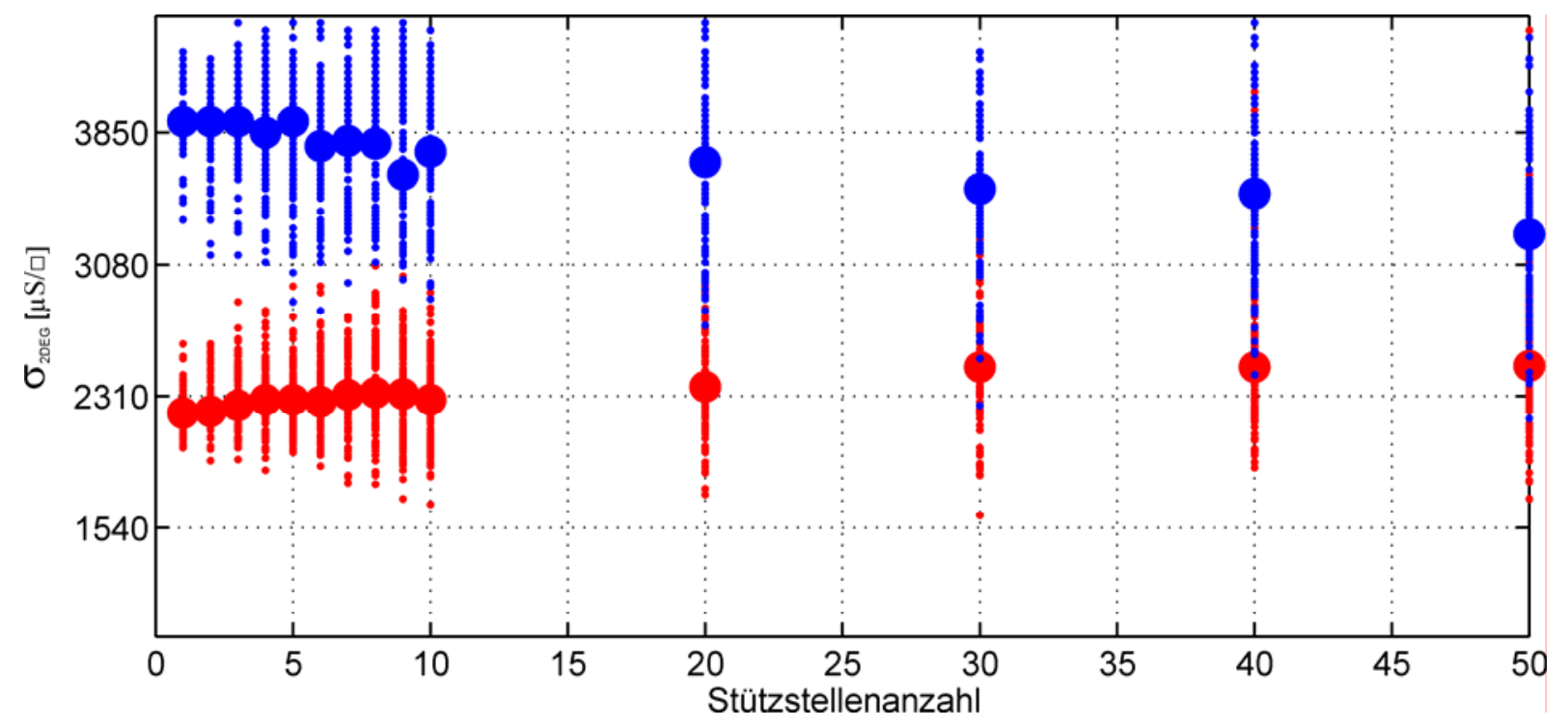

Abbildung 76: Ergebnisse der Leitfähigkeitsanpassung bei einem Testpotential mit Linienabzug (blau). Der Medianwert der 2DEG-Leitfähigkeiten aller Konfigurationen (blau) ist $3660 \pm 1090 \mu \mathrm{S} / \square$. In Rot sind Ergebnisse für den invertierten Linienabzug dargestellt. Der Medianwert aller Verhältnisse (rot) ist bei dieser Verfremdung der Testpotentiallandschaft $2460 \pm 380 \mu S / \square$. Der Mittelwert ist somit $3060 \mu S / \square$ und entspricht sehr exakt der eingesetzten 2DEG-Leitfähigkeit.

Die Verwendung zusätzlicher Stützstellen ist somit geeignet den Einfluss von linienartigen Störungen die durch den Linienabzug der Nachbearbeitung oder aber durch Störungen während der Messung auftreten, zu verringern. Es wurde gezeigt, dass sich mit zunehmender Stützstellenanzahl die angepasste 2DEG-Leitfähigkeit der eingesetzten 2DEG-Leitfähigkeit annähert. Im Rahmen der hier präsentierten Analyse erscheint es ferner gerechtfertigt die 
Standardabweichung aus der Zufallsverteilung der Stützstellenpositionen als Maß für den Fehler der Bestimmung zu verwenden.

\section{Bearbeitung der Messdaten durch Ebenenabzug}

Die Veränderung der Raumtemperatur (Tag/Nachtabsenkung der Vorlauftemperatur der Heizkörper) erzeugt häufig ein Störsignal. In den $\mu_{\mathrm{ec}, \mathrm{STP}}(\mathrm{x}, \mathrm{y})$-Daten zeigt sich diese Störung als „Tonne“ entlang der langsamen Rasterrichtung. Der bekannten Testpotential wurde eine quadratische Hyperfläche überlagert um diesem Sachverhalt zu entsprechen (siehe Abbildung 77(b)). Das neue Testpotential zeigt Abbildung 77(c).

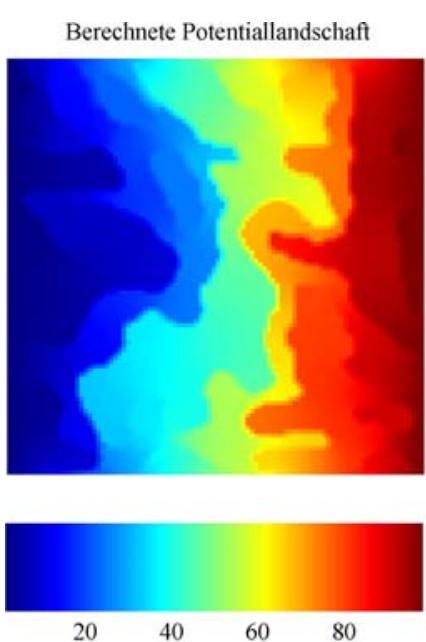

a)

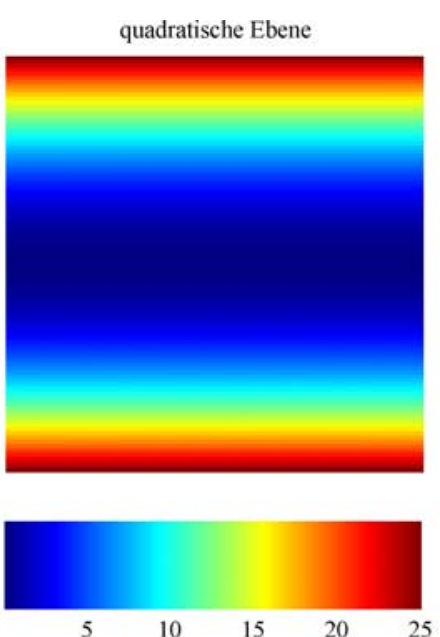

b)

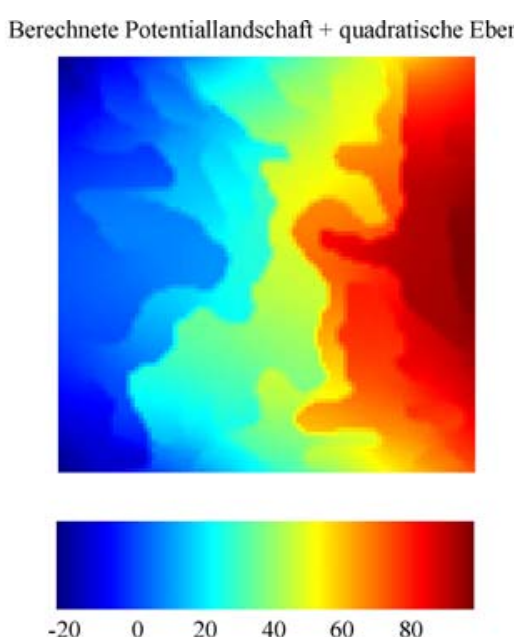

c)

Abbildung 77: Dem bereits verwendetem Testpotential aus dem vorherigen Abschnitt (a) wird eine quadratische Ebene (b) abgezogen. Das neue Testpotential (c) zeigt dadurch eine tonnenartige Störung entlang der langsamen Rasterrichtung, welche häufig in den Messdaten zu finden sind (Vergleiche Abschnitt 3.2).

Ergebnisse der Leitfähigkeitsanpassung mit diesem Testpotential zeigt Abbildung 78. Es ist deutlich zu sehen, dass die 2DEG-Leitfähigkeit ab 20 zusätzlichen Stützstellen nahezu konstant ist. Bei 50 zusätzlichen Stützstellen wird die 2DEG-Leitfähigkeit zu $2190 \pm 1030$ $\mu \mathrm{S} / \square$ bzw. $2120 \pm 620 \mu \mathrm{S} / \square$ (Hyperfläche invertiert) bestimmt. 


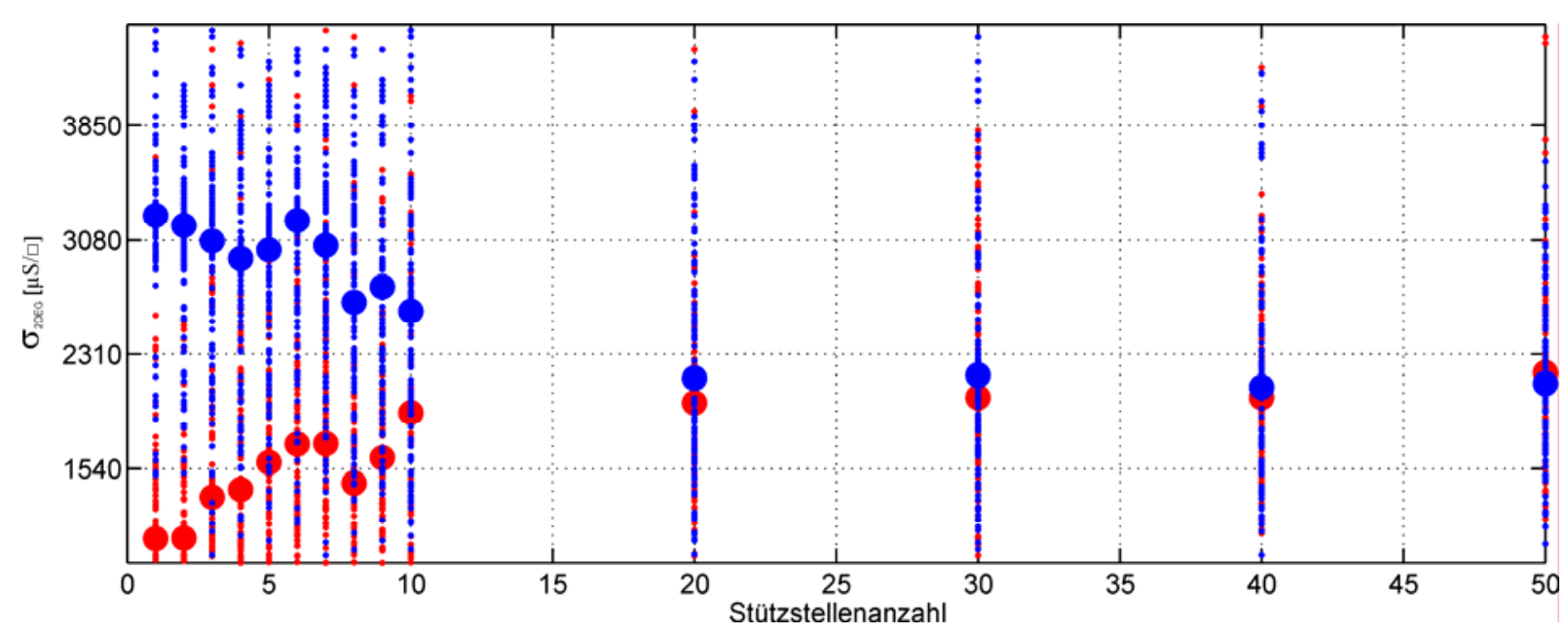

Abbildung 78: Ergebnisse der Leitfähigkeitsanpassung: Der Medianwert der 2DEGLeitfähigkeiten aller Konfigurationen (blau) ist $2880 \pm 1850 \mu \mathrm{S} / \square$. In Rot sind Ergebnisse für den invertierten Hyperflächenabzug dargestellt. Ihr Medianwert (rot) ist bei dieser Verfremdung der Testpotentiallandschaft $1870 \pm 870 \mu \mathrm{S} / \square$. Bei 50 zusätzlichen Stützstellen wird die 2DEG-Leitfähigkeit zu $2190 \pm 1030 \mu S / \square$ (rot) bzw. $2120 \pm 620 \mu S / \square$ (blau) bestimmt.

Zusammenfassend konnte die eingesetzte 2DEG-Leitfähigkeit bei allen Konsistenzüberprüfungen gut reproduziert werden. Leider ist bei experimentellen Datensätzen die Störung unbekannt und liegt darüber hinaus nicht in seiner invertierten Form vor, so dass bei der Analyse der Leitfähigkeiten die Standardabweichung als ein Maß für die Ungenauigkeit der Leitfähigkeitsbestimmung verwendet werden muss. 


\section{Literaturverzeichnis}

[1] Rutherglen, C. and Burke, P. Nano Lett. 7(11), 3296-3299 November (2007).

[2] Jensen, K., Weldon, J., Garcia, H., and Zettl, A. Nano Lett. 7(11), 3508-3511 November (2007).

[3] Welter, K. Chemie in unserer Zeit 42(1), 8-9 (2008).

[4] http://www.itrs.net, (2007).

[5] Roy, S. and Asenov, A. Science 309, 388 (2005).

[6] Rau, W. D., Schwander, P., Baumann, F. H., Höppner, W., and Ourmazd, A. Phys. Rev. Lett. 82(12), 2614 March (1999).

[7] Muralt, P. and Pohl, D. W. Applied Physics Letters 48(8), 514-516 (1986).

[8] Schneider, M. A., Wenderoth, M., Heinrich, A. J., Rosentreter, M. A., and Ulbrich, R. G. Appl. Phys. Lett. 69(9), 1327-1329 August (1996).

[9] Vries, J. W. C. d. Journal of Physics F: Metal Physics 17(9), 1945-1952 (1987).

[10] Kirtley J. R., Washburn S., B. M. J. IBM Journal of Research and Development 32, 414 (1988).

[11] Kirtley, J. R., Washburn, S., and Brady, M. J. Phys. Rev. Lett. 60(15), 1546April (1988).

[12] Pelz, J. P. and Koch, R. H. Phys. Rev. B 41(2), 1212- January (1990).

[13] Pelz, J. P. and Koch, R. H. Review of Scientific Instruments 60(3), 301-305 (1989).

[14] Hamers, R. J. and Markert, K. Phys. Rev. Lett. 64(9), 1051- February (1990).

[15] Besold, J., Reiss, G., and Hoffmann, H. Applied Surface Science 65-66, 23-27 March (1993).

[16] Briner, B. G., Feenstra, R. M., Chin, T. P., and Woodall, J. M. Phys. Rev. B 54(8), R5283- August (1996).

[17] Briner, B. G., Feenstra, R. M., Chin, T. P., and Woodall, J. M. Semiconductor Science and Technology 11(11S), 1575-1581 (1996).

[18] Ramaswamy, G. and Raychaudhuri, A. K. Appl. Phys. Lett. 75(13), 1982-1984 September (1999). 
[19] Paranjape, M., Raychaudhuri, A. K., Mathur, N. D., and Blamire, M. G. Phys. Rev. B 67(21), 214415- June (2003).

[20] Grévin, B., Maggio-Aprile, I., Bentzen, A., Ranno, L., Llobet, A., and Fischer, . Phys. Rev. B 62(13), 8596- October (2000).

[21] Ramaswamy, G., Raychaudhuri, A., Das Gupta, K., and Sambandamurthy, G. Applied Physics A: Materials Science \& Processing 66(0), S435-S439 March (1998).

[22] Bannani, A. Ladungstransport in dünnen, metallischen Filmen. $\mathrm{PhD}$ thesis, Universität Duisburg-Essen, (2007).

[23] Ashcroft, N. W. and Mermin, N. D. Festkörperphysik. Oldenbourg Verlag München Wien, (2001).

[24] Bardeen, J. Phys. Rev. Lett. 6, 57-59 (1961).

[25] Chen, J. Introduction to Scanning Tunneling Microscopy. Oxford University Press, (1993).

[26] Tersoff, J. Theory of Scanning Tunneling Microscopy. Scanning Probe Microscopy and Spectroscopy. Wiley-VCH New York, (2001).

[27] Tersoff, J. Phys. Rev. B 40(17), 11990-December (1989).

[28] Tersoff, J. and Hamann, D. R. Phys. Rev. B 31(2), 805- January (1985).

[29] Tersoff, J. and Hamann, D. R. Phys. Rev. Lett. 50(25), 1998- June (1983).

[30] Engel, K. J. Scanning-Thermovoltage-Microscopy of the interference of electron waves on the Au(111)-surface. PhD thesis, Universität Göttingen, IV. Physikalisches Institut, (2001).

[31] Lang, N. D. Phys. Rev. B 34(8), 5947- October (1986).

[32] Schneider, M. A. Rastertunnelmikroskop-Untersuchungen der Streuung von Elektronen an Defekten am Beispiel dünner Au-Filme. $\mathrm{PhD}$ thesis, Universität Göttingen, (1997).

[33] Müller, J. Master's thesis, Universität Göttingen, (2003).

[34] Homoth, J., Wenderoth, M., Engel, K. J., Druga, T., Loth, S., and Ulbrich, R. G. Phys. Rev. B 76(19), 193407-4 November (2007). 
[35] Loth, S. Atomic scale images of acceptors in III-V semiconductors: band bending, tunneling paths and wave functions. $\mathrm{PhD}$ thesis, Georg-August Universität Göttingen, (2008).

[36] Druga, T. C. Diplomarbeit, IV. Physikalischen Institut der Georg-AugustUniversität zu Göttingen, (2007).

[37] Rosentreter, M. A. Master's thesis, Universität Göttingen, (1993).

[38] Quast, T. Master's thesis, University of Göttingen, (1995).

[39] Datta, S. Electronic Transport in Mesoscopic Systems. Cambridge Studies in Semiconductor Physics and Microelectronic Engeneering, (2003).

[40] Kittel, C. Einführung in die Festkörperphysik. R.Oldenbourg Verlag München Wien, (1999).

[41] van Wees, B. J., van Houten, H., Beenakker, C. W. J., Williamson, J. G., Kouwenhoven, L. P., van der Marel, D., and Foxon, C. T. Phys. Rev. Lett. 60(9), 848- February (1988).

[42] Imry, Y. and Landauer, R. Rev. Mod. Phys. 71(2), S306- March (1999).

[43] Landauer, R. IBM J. Res. Dev. 32(3), 306-316 (1988).

[44] Landauer, R. Physica Scripta T42, 110-114 (1992).

[45] Landauer, R. IBM J. Res. Dev. 1, 223-231 (1957).

[46] Zwerger, W., Bönig, L., and Schönhammer, K. Phys. Rev. B 43(8), 6434- March (1991).

[47] Birk, H., de Jong, M. J. M., and Schönenberger, C. Phys. Rev. Lett. 75(8), 1610August (1995).

[48] van der Ziel, A. Noise in Solid State Devices and Circuits. Wiley, New York, (1986).

[49] Koslowski, B. and Baur, C. J. Appl. Phys. 77(1), 28-33 January (1995).

[50] Möller, R., Baur, C., Esslinger, A., and Kurz, P. In J. Vac. Sci. Technol. B, volume 9, 609-611 (AVS, Baltimore, Massachusetts (USA), 1991).

[51] Möller, R., Esslinger, A., and Koslowski, B. J. Vac. Sci. Technol. A 8(1), 590593 January (1990). 
[52] Möller, R., Esslinger, A., and Koslowski, B. Appl. Phys. Lett. 55(22), 2360-2362 November (1989).

[53] Johnson, J. B. Phys. Rev. 32(1), 97- July (1928).

[54] Nyquist, H. Phys. Rev. 32(1), 110- July (1928).

[55] Hsu, S.T.; Whittier, R. Electron Devices Meeting, 1969 International 15, 86-88 (1969).

[56] Stövneng, J. A. and Lipavský, P. Phys. Rev. B 42(14), 9214- November (1990).

[57] Hoffmann, D., Grand, J. Y., Möller, R., Rettenberger, A., and Läuger, K. Phys. Rev. B 52(19), 13796- November (1995).

[58] Hoffmann, D., Seifritz, J., Weyers, B., and Möller, R. Journal of Electron Spectroscopy and Related Phenomena 109(1-2), 117-125 August (2000).

[59] Engel, K. J., Wenderoth, M., Quaas, N., Reusch, T. C. G., Sauthoff, K., and Ulbrich, R. G. Phys. Rev. B 63(16), 165402- March (2001).

[60] Williams, C. C. and Wickramasinghe, H. K. Nature 344(6264), 317-319 March (1990).

[61] Herman, G. S., Woicik, J. C., Andrews, A. B., and Erskine, J. L. Surface Science 290(1-2), L643-L648 June (1993).

[62] Kono, S., Higashiyama, K., Kinoshita, T., Miyahara, T., Kato, H., Ohsawa, H., Enta, Y., Maeda, F., and Yaegashi, Y. Phys. Rev. Lett. 58(15), 1555- April (1987).

[63] Nakajima, Y., Takeda, S., Nagao, T., Hasegawa, S., and Tong, X. Phys. Rev. B 56(11), 6782- September (1997).

[64] Nicholls, J. M., Salvan, F., and Reihl, B. Phys. Rev. B 34(4), 2945- August (1986).

[65] Zhang, H. M., Gustafsson, J. B., and Johansson, L. S. O. Phys. Rev. B 74(20), 201304-4 November (2006).

[66] Ding, Y. G., Chan, C. T., and Ho, K. M. Phys. Rev. Lett. 67(11), 1454 September (1991).

[67] Watanabe, S., Aono, M., and Tsukada, M. Phys. Rev. B 44(15), 8330- October (1991). 
[68] Carpinelli, J. M. and Weitering, H. H. Phys. Rev. B 53(19), 12651- May (1996).

[69] Demuth, J. E., Von Lenen, E. J., Tromp, R. M., and Hamers, R. J. J. Vac. Sci. Technol. B 6(1), 18-25 January (1988).

[70] McComb, D. W., Moffatt, D. J., Hackett, P. A., Williams, B. R., and Mason, B. F. Phys. Rev. B 49(24), 17139- June (1994).

[71] van Loenen, E. J., Demuth, J. E., Tromp, R. M., and Hamers, R. J. Phys. Rev. Lett. 58(4), 373- January (1987).

[72] Wan, K. J., Lin, X. F., and Nogami, J. Phys. Rev. B 45(16), 9509-April (1992).

[73] Wan, K. J., Lin, X. F., and Nogami, J. Phys. Rev. B 47(20), 13700- May (1993).

[74] Wilson, R. J. and Chiang, S. Phys. Rev. Lett. 58(4), 369- January (1987).

[75] Borensztein, Y. and Alameh, R. Surface Science 274(1), L509-L514 July (1992).

[76] Hasegawa, S. and Ino, S. Surface Science 283(1-3), 438-446 March (1993).

[77] Hasegawa, S., Tong, X., Jiang, C.-S., Nakajima, Y., and Nagao, T. Surface Science 386(1-3), 322-327 October (1997).

[78] Jiang, C.-S., Hasegawa, S., and Ino, S. Phys. Rev. B 54(15), 10389- October (1996).

[79] Schad, R., Heun, S., Heidenblut, T., and Henzler, M. Phys. Rev. B 45(19), 11430- May (1992).

[80] Takahashi, T. and Nakatani, S. Surface Science 282(1-2), 17-32 February (1993).

[81] Matsuda, I., Morikawa, H., Liu, C., Ohuchi, S., Hasegawa, S., Okuda, T., Kinoshita, T., Ottaviani, C., Cricenti, A., D'angelo, M., Soukiassian, P., and Lay, G. L. Phys. Rev. B 68, 085407 (2003).

[82] Nakahara, H., Suzuki, T., and Ichimiya, A. Appl. Surf. Sci. 234, 292-296 (2004).

[83] Sato, N., Nagao, T., and Hasegawa, S. Surface Science 442, 65-73 (1999).

[84] Takahashi, T., Tajiri, H., Sumitani, K., Akimoto, K., Sugiyama, H., Zhang, X., and Kawata, H. Surf. Rev. Let. 10, 519-524 (2003).

[85] Kuwahara, Y., Nakatani, S., Takahasi, M., Aono, M., and Takahashi, T. Surface Science 310(1-3), 226-230 May (1994). 
[86] Aizawa, H., Tsukada, M., Sato, N., and Hasegawa, S. Surface Science 429(1-3), L509-L514 June (1999).

[87] Sato, N., Takeda, S., Nagao, T., and Hasegawa, S. Phys. Rev. B 59(3), 2035January (1999).

[88] Nakamura, Y., Kondo, Y., Nakamura, J., and Watanabe, S. Phys. Rev. Lett. 87(15), 156102- September (2001).

[89] Crain, J. N., Altmann, K. N., Bromberger, C., and Himpsel, F. J. Phys. Rev. B 66(20), 205302- November (2002).

[90] Crain, J. N., Gallagher, M. C., McChesney, J. L., Bissen, M., and Himpsel, F. J. Phys. Rev. B 72(4), 045312-4 July (2005).

[91] Uhrberg, R. I. G., Zhang, H. M., Balasubramanian, T., Landemark, E., and Yeom, H. W. Phys. Rev. B 65(8), 081305- February (2002).

[92] Matsuda, I., Hirahara, T., Konishi, M., Liu, C., Morikawa, H., D'angelo, M., Hasegawa, S., Okuda, T., and Kinoshita, T. Phys. Rev. B 71(23), 235315-11 June (2005).

[93] Sato, N., Nagao, T., and Hasegawa, S. Phys. Rev. B 60, 16083-16087 (1999).

[94] Tong, X., Jiang, C. S., Horikoshi, K., and Hasegawa, S. Surface Science 449(13), 125-134 March (2000).

[95] Viernow, J., Henzler, M., O'Brien, W. L., Men, F. K., Leibsle, F. M., Petrovykh, D. Y., Lin, J. L., and Himpsel, F. J. Phys. Rev. B 57(4), 2321- January (1998).

[96] Hirahara, T., Matsuda, I., Ueno, M., and Hasegawa, S. Surface Science 563(1-3), 191-198 August (2004).

[97] Tagayanagi, K., Tanishiro, Y., Takahashi, M., and Takahashi, S. Surface Science 164, 367-392 (1985).

[98] Hamers, R. J., Tromp, R. M., and Demuth, J. E. Surf. Sci. 181, 346 (1987).

[99] Ono, M., Nishigata, Y., Nishio, T., Eguchi, T., and Hasegawa, Y. Phys. Rev. Lett. 96(1), 016801-4 January (2006).

[100] Watanabe, S., Kondo, Y., Nakamura, Y., and Nakamura, J. 1, 167-172 (2000).

[101] Ebert, P., Jager, N. D., Urban, K., and Weber, E. R. J. Vac. Sci. Technol. B 22(4), 2018-2025 July (2004). 
[102] McComb, D. W., Wolkow, R. A., and Hackett, P. A. Phys. Rev. B 50(24), 18268December (1994).

[103] Oura, K., Lifshits, V. G., Saranin, A. A., Zotov, A. V., and Katayama, M. Surface Science An Introduction. Springer-Verlag Berlin Heidelberg New York, (2003).

[104] Saranin, A. A., Zotov, A. V., Lifshits, V. G., Ryu, J. T., Kubo, O., Tani, H., Harada, T., Katayama, M., and Oura, K. Surface Science 429(1-3), 127-132 June (1999).

[105] Matsuda, I., Liu, C., Hirahara, T., Ueno, M., Tanikawa, T., Kanagawa, T., Hobara, R., Yamazaki, S., Hasegawa, S., and Kobayashi, K. Phys. Rev. Lett. 99(14), 146805-4 October (2007).

[106] Hasegawa, S., Shiraki, I., Tanikawa, T., Petersen, C. L., Hansen, T. M., Boggild, P., and Grey, F. Journal of Physics: Condensed Matter 14(35), 8379-8392 (2002).

[107] Hasegawa, S., Shiraki, I., Tanabe, F., Hobara, R., Kanagawa, T., Tanikawa, T., Matsuda, I., Petersen, C. L., Hansen, T. M., Boggild, P., and Grey, F. Surface Review and Letters 10, 963-980 (2003).

[108] Wells, J. W., Kallehauge, J. F., and Hofmann, P. Journal of Physics: Condensed Matter 19(17), 176008 (7pp) (2007).

[109] Hasegawa, S., Tong, X., Takeda, S., Sato, N., and Nagao, T. Progress in Surface Science 60(5-8), 89-257 March (1999).

[110] Nakajima, Y., Uchida, G., Nagao, T., and Hasegawa, S. Phys. Rev. B 54(19), 14134- November (1996).

[111] Neddermeyer, H. Reports on Progress in Physics 59(6), 701-769 (1996).

[112] Noffsinger, J. Physics REU Program Summer 2003, (2003).

[113] Viernow, J., Lin, J.-L., Petrovykh, D. Y., Leibsle, F. M., Men, F. K., and Himpsel, F. J. Appl. Phys. Lett. 72(8), 948-950 February (1998).

[114] Matsuda, I., Ueno, M., Hirahara, T., Hobara, R., Morikawa, H., Liu, C., and Hasegawa, S. Phys. Rev. Lett. 93(23), 236801-4 December (2004).

[115] Matsuda, I., Hirahara, T., Ueno, M., Hobara, R., and Hasegawa, S. J. Phys. IV France 132, 57-61 (2006). 
[116] Schüssler, H., W. Netzwerke, Signale und Systeme. Springer-Verlag, 3. auflage edition, (1991).

[117] Unbehauen, R. Elektrische Netzwerke. Springer-Verlag, 3.auflage edition, (1990).

[118] Lagarias, J. C., Reeds, J. A., Wright, M. H., and Wright, P. E. SIAM Journal on Optimization 9(1), 112-147 (1998).

[119] Zubkov, E. Master's thesis, Universität Duisburg-Essen, Institut für Experimentelle Physik, (2008).

[120] Sondheimer, E. H. Advances in Physics 1(1), 1-42 (1952).

[121] Friedel, J. Il Nuovo Cimento (1955-1965) 7(0), 287-311 September (1958).

[122] Lang, N. D. and Kohn, W. Phys. Rev. B 1(12), 4555- June (1970).

[123] Schleiermacher, H. Master's thesis, (2006).

[124] Kobayashi, K. Surface Science 583(1), 16-28 May (2005). 


\section{Abkürzungsverzeichnis}

ARUPS Angel Resolved Ultraviolet Photoemission Spectroscopy

$\mathrm{E}_{\mathrm{f}} \quad$ Fermienergie

$\mathrm{E}_{0} \quad$ Minimum des Oberflächenzustands $\mathrm{S}_{1}$

HCT „honeycomb-chained-trimer“

IET ,inequivalent-trimer“

$\mathrm{k}_{\mathrm{f}} \quad$ Fermiwellenzahl

LBM Leitungsbandminimum

LDOS Lokale Zustandsdichte (engl. Local Density of States)

LEED Low Energy Electron Diffraction

$\lambda_{\mathrm{f}} \quad$ Fermiwellenlänge

OVBM Oberflächenvalenzbandmaximum

STM Raster-Tunnel-Mikroskopie (engl. Scanning Tunneling Microscopy, STM)

STP Raster-Tunnel-Potentiometrie (engl. Scanning Tunneling Potentiometry, STP)

$\mathrm{U}_{\text {Bias }} \quad$ elektrische Spannung am Tunnelkontakt $U_{\text {Bias }}=U_{\text {Probe }}-U_{\text {Spitze }}$

UHV Ultra-Hoch-Vakuum, Druckbereich geringer als $10^{-9} \mathrm{mBar}$

$\mu_{\mathrm{c}} \quad$ Das chemische Potential

$\mu_{\mathrm{ec}} \quad$ Das elektrochemische Potential: $\mu_{e c}(x, y)=\mu_{c}(x, y)-e \phi(x, y)$

$\mu_{\mathrm{ec}, \mathrm{STP}} \quad$ Messgröße der Raster-Tunnel-Potentiometrie (Kapitel 1.2 und 1.1)

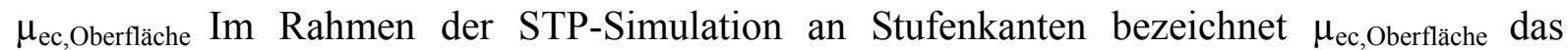
elektrochemische Potential der Probe.

VBM Valenzbandmaximum 


\section{Publikationen und Präsentationen}

\section{Publikationen:}

L. Winking, M. Wenderoth, J. Homoth, S. Siewers, R. G. Ulbrich, Fe films grown on

GaAs(110) in a two-step process: Improved structural and magnetic properties, Appl. Phys. Lett., 92(19):193102, May 2008.

J. Homoth, M. Wenderoth, K. J. Engel, T. Druga, S. Loth, R. G. Ulbrich, Reconstruction of the local density of states in Ag(111) surfaces using Scanning Tunneling Potentiometry, Phys. Rev. B, 76(19):193407, November 2007.

M. Bertelli, J. Homoth, M. Wenderoth, A. Rizzi, R. G. Ulbrich, M. C. Righi, C. M. Bertoni, A. Catellani, Atomic and electronic structure of the cleaved $6 H-S i C(11 \overline{2} 0)$ surface, Phys. Rev. B, 75(16):165312, April 2007.

J. Müller, M. Wenderoth, N. Quaas, T. C. G. Reusch, R. G. Ulbrich, Evolution of thin Cu films on GaAs(110) towards atomically flat epitaxial overlayers,

Appl. Phys. Lett., 85(12):2220, September 2004.

\section{Präsentationen:}

2007 "Importance of thermovoltage on the interpretation of STM data", "Nanoscale imaging of the transport field in a current-carrying two-dimensional electron gas" und "Tunneling spectroscopy of an operating pin-diode interface", International Conference IVC17, ICSS13, ICNT2007 2007(Stockholm)

2006 "Scanning Tunnelling Potentiometry of Ag films on GaAs(110)", International Conference on Scanning Tunneling Microscopy 2006 (Basel)

"Electrical fields of planar defects in percolation structures: Scanning Tunnelling Potentiometry of $\mathrm{Ag}$ films on GaAs(110)", International Conference on Surface Science ECOSS 2006 (Paris) 
"STM-investigations of metal films near the percolation threshold on GaAs(110)" und „Imaging of local potentials and thermovoltages with the STM”, Frühjahrstagung der Deutschen Physikalischen Gesellschaft (DPG) (Dresden)

2005 "STM-investigation of a nanocluster $\mathrm{Cu}$ film on $\operatorname{GaAs}(110)$ ", Frühjahrstagung der DPG (Berlin)

"Electronic growth of thin $\mathrm{Cu}$-films on GaAs(110): A long-time STM-study", NANO 8 (Venedig)

"Epitaxial Growth of thin, atomically flat Cu-Films on GaAs(110)", Frühjahrstagung der DPG (Regensburg)

2003 „Rastertunnelmikroskopie an dünnen epitaktischen Cu-Filmen auf $\mathrm{GaAs}(110)^{\text {“ }}$ Frühjahrstagung der DPG (Dresden) 


\section{Danksagung}

Experimentelle Forschung kann man nicht im Alleingang bewerkstelligen und viele Dinge gelingen erst durch das richtige Teamwork. Darum möchte ich hier die Gelegenheit nutzen, allen Personen, die mir während der vergangenen Jahre mit Rat und Tat zur Seite gestanden haben, herzlichst zu danken.

An erster Stelle möchte ich Prof. Dr. Rainer G. Ulbrich danken, den ich gleich zu Beginn meiner Studienzeit in Göttingen kennengelernt habe und seitdem als kompetenten Ratgeber schätze. Er wird mir mit seinem steten Wunsch, die physikalischen Phänomene auf grundlegender Ebene zu verstehen, ein Vorbild bleiben.

Prof. Dr. K. Samwer möchte ich herzlichst für die Übernahme des Korreferats danken.

Prof. Dr. R. Möller, sowie seinen Mitarbeitern, danke ich für die vielen fruchtbaren Diskussionen und die gute Zusammenarbeit. Es war für mich eine große Freude, die erarbeiteten Kenntnisse und Modellierungen im Rahmen einer Kooperation auf vizinale Oberflächen anwenden zu dürfen. Seine Anregungen und Kritiken haben sehr zur Ausarbeitung der in Abschnitt 3.3 vorgestellten Ergebnisse beigetragen.

Ein besonderes Dankeschön möchte ich an Dr. Martin Wenderoth richten. In den Phasen meiner Dissertation, wo nichts klappte und die Technik mir einen Streich spielte, war er mir nicht nur ein hervorragender Mentor, sondern auch ein aufmunternder Freund. Seine physikalische Herangehensweise hat mich tief geprägt und wird mir auch bei zukünftigen Herausforderungen zur Seite stehen.

Auch an den Rest der STM-Arbeitsgruppe werde ich mich gerne erinnern - die ehemaligen und neuen Tunnler: Thomas Druga, Klaus Engel, Jens Garleff, Peter Löptin, Karolin Löser, Sebastian Loth, Henning Prüser, Thilo Reusch, Katharina Sauthoff, Swante Siewers, Bernhard Spicher, Karen Teichmann, Norbert Quaas und Alexander Weismann. Wir hatten eine spannende Zeit und haben gemeinsam viel erreicht. Die unzähligen gemeinsamen Kaffeepausen mit Diskussionen über die Physik und den Rest der Welt waren immer angenehme Muntermacher. Insbesondere möchte ich einen herzlichen Dank an Thomas Druga richten. Es war eine enorme Entlastung, die aufwendigen Messkampagnen zusammen mit einem engagierten Diplomanden vorbereiten und durchführen zu können. 
Ebenfalls hat mich Bernhard Spicher durch seine unverzichtbaren Ingenieurkenntnisse vortrefflich unterstützt. Seine ureigene Art trägt seit langem zum Erfolg der jeweiligen Projekte, aber auch zur Laborharmonie bei. Der Feinmechanik- sowie Elektrowerkstatt des IV. Physikalischen Instituts möchte ich für die gute Unterstützung bei allen technischen Dingen des Laboralltags danken.

Nicht vergessen möchte ich meinen langjährigen Mitstreiter und guten Freund Lars Winking. Nur durch die gegenseitige Unterstützung gelang es uns von der Anfängervorlesung bis jetzt, der Physik stets die Stirn zu bieten.

Vor allem bin ich meiner lieben Familie zutiefst dankbar. Es ist ein gutes Gefühl zu wissen, dass ich mich in allen Lebenslagen auf ihre Unterstützung und unseren engen Zusammenhalt verlassen kann. Meinen Eltern gebührt ein ebenso großer Dank für die vielen aufmunternden Gespräche und die in mich investierte Zeit. Sie haben meinen Wissensdurst stets gefördert und mich bestärkt nach dem „Warum der Dinge“ zu fragen. 


\title{
Lebenslauf
}

\author{
Jan Homoth, geb. Müller \\ Geboren am 26.04.1977 in Göttingen \\ Staatsangehörigkeit: deutsch
}

\begin{abstract}
$1984-1988$
Herman-Nohl Grundschule (Göttingen)

$1988-1990$

Berthold-Brecht Orientierungsstufe (Göttingen)

$1990-1997$

Hainberg Gymnasium (Göttingen)

$10 / 1998-7 / 2003$

Diplomstudiengang Physik an der Universität Göttingen

$1 / 2002-7 / 2003$

Diplomarbeit am Institut für Halbleiterphysik der Universität Göttingen mit dem Titel „Herstellung und Charakterisierung ultradünner $\mathrm{Cu}$-Filme auf der $\mathrm{GaAs}(110)$ Spaltfläche für Ladungstransportmessung mit dem Rastertunnelmikroskop"

$8 / 2003-07 / 2008$

Promotion am Institut für Halbleiterphysik der Universität Göttingen mit dem Titel "Das elektrochemische Potential auf der atomaren Skala: Untersuchung des Ladungstransports eines stromtragenden zweidimensionalen Elektronengases mit Hilfe der Raster-Tunnel-Potentiometrie“
\end{abstract}

Göttingen den 11.06.2008 